Intracellular Quantification and Pharmacokinetics of Pyrimidine Analogues used in Oncology 
ISBN/EAN: 978-94-6375-689-1

Copyright $($ C, 2019 Ellen Derissen

Artwork cover: $\quad$ Antonia Verbrugge

Lay-out: $\quad$ Renske Hortensius | persoonlijkproefschrift.nl

Printed by: $\quad$ Ridderprint BV | www.ridderprint.nl

No part of this thesis may be reproduced, stored in a retrieval system or transmitted in any form or by any means without prior permission of the author. 


\title{
Intracellular Quantification and Pharmacokinetics of Pyrimidine Analogues used in Oncology
}

\author{
Intracellulaire Kwantificering en Farmacokinetiek \\ van Pyrimidine Analoga toegepast in de Oncologie \\ (met een samenvatting in het Nederlands)
}

\begin{abstract}
Proefschrift
ter verkrijging van de graad van doctor aan de Universiteit Utrecht op gezag van de rector magnificus, prof. dr. H.R.B.M. Kummeling, ingevolge het besluit van het college voor promoties in het openbaar te verdedigen op woensdag 29 januari 2020 des middags te 12.45 uur
\end{abstract}

door

Eleonora Julie Bertine Derissen

geboren op 23 januari 1984 te Zeist 
Promotor:

Copromotor:
Prof. dr. J.H. Beijnen

Dr. H. Rosing 
The research described in this thesis was performed at the Department of Pharmacy \& Pharmacology and the Department of Clinical Pharmacology of the Netherlands Cancer Institute - Antoni van Leeuwenhoek hospital, Amsterdam, the Netherlands.

Printing of this thesis was financially supported by the Netherlands Cancer Institute. 


\section{CONTENTS}

Preface

Chapter 1: Intracellular Pharmacokinetics of Pyrimidine

Analogues used in Oncology and the Correlation with Drug Action

Submitted for Publication

Chapter 2: Azacitidine and Decitabine

2.1 Concise drug review: azacitidine and decitabine

The Oncologist 2013;18:619-624.

2.2 Quantitative determination of azacitidine triphosphate in peripheral blood mononuclear cells using liquid chromatography coupled with high-resolution mass spectrometry

Journal of Pharmaceutical and Biomedical Analysis 2014;90:7-14.

Chapter 3: 5-Fluorouracil and Capecitabine

3.1 Development of an LC-MS/MS assay for the quantitative determination of the intracellular 5-fluorouracil nucleotides responsible for the anticancer effect of 5-fluorouracil Journal of Pharmaceutical and Biomedical Analysis 2015;110:58-66.

3.2 Exploring the intracellular pharmacokinetics of the 5-fluorouracil nucleotides during capecitabine treatment

British Journal of Clinical Pharmacology 2016;81:949-957. 


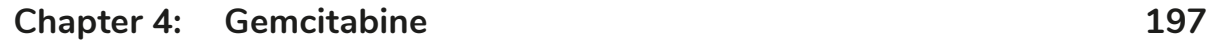

4.1 Intracellular pharmacokinetics of gemcitabine, its 199 deaminated metabolite 2',2'-difluorodeoxyuridine and their nucleotides

British Journal of Clinical Pharmacology 2018;84:1279-1289.

Summary, Conclusions and Perspectives

Samenvatting, Conclusies en Perspectieven

Chemical Structures of the Pyrimidine Analogues discussed in this Thesis

List of Publications

Dankwoord

Curriculum Vitae 


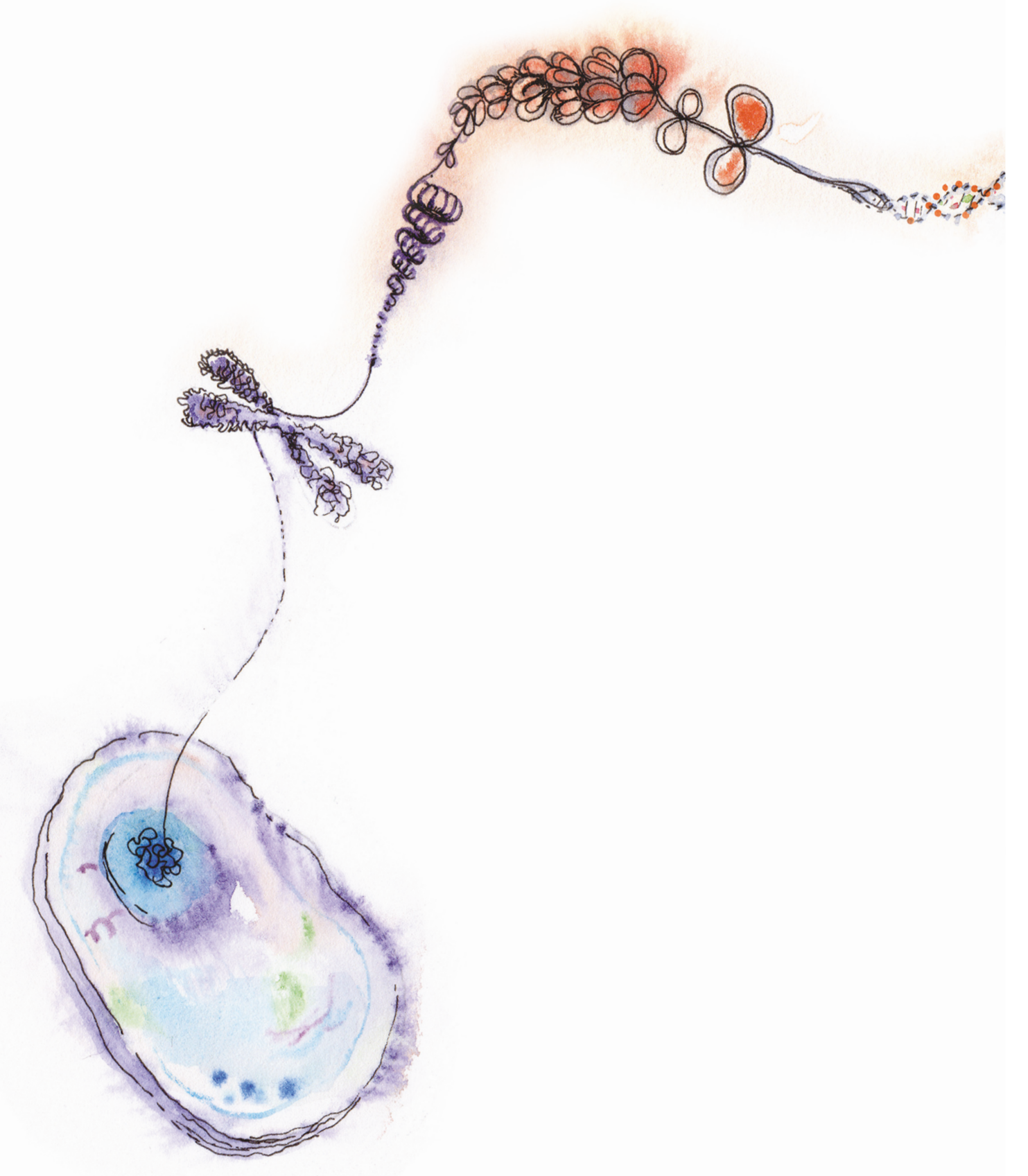


मों

in

$\because 4: 5$

PREFACE

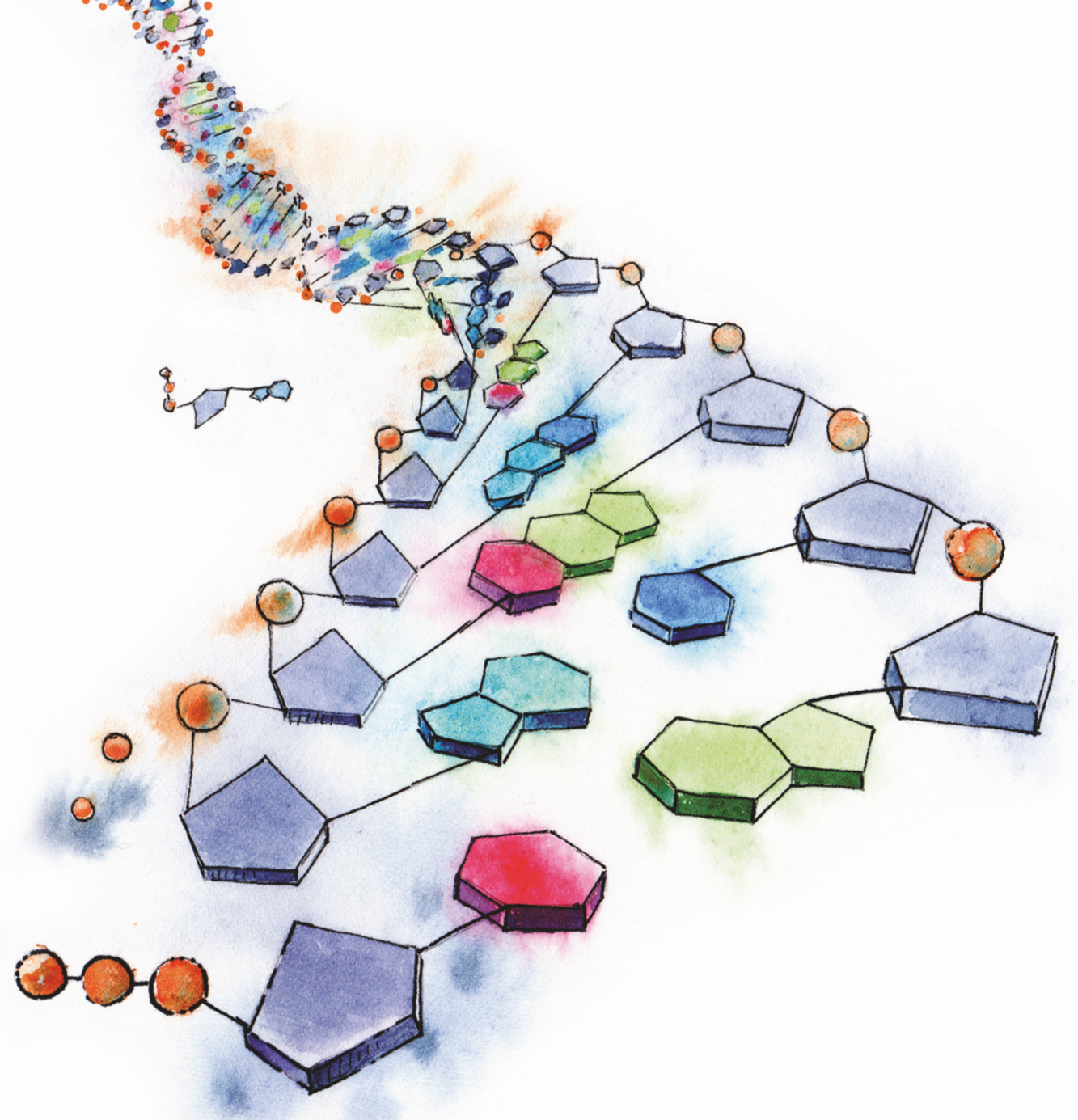




\section{PREFACE}

Pyrimidine analogues form a classical group of cytostatic drugs that still have an important place in the treatment of various solid and haematological malignancies. Pyrimidine analogues are structural analogues of the natural pyrimidine building blocks of DNA and RNA. Pyrimidine analogues are therefore analogues of the natural nucleobases cytosine, uracil and thymine or of their derived nucleosides cytidine, deoxycytidine, uridine and thymidine. Because of the structural modifications with respect to the natural pyrimidines, pyrimidine analogues disrupt natural processes such as DNA and RNA synthesis and DNA repair. This ultimately leads to the death of the tumour cell.

There are currently eight oncolytic pyrimidine analogues on the market in the Netherlands: the uracil analogue 5-fluorouracil (5-FU) and its prodrugs capecitabine and tegafur, the cytidine analogue azacitidine, the deoxycytidine analogues cytarabine, gemcitabine and decitabine, and the thymidine analogue trifluridine [1,2]. An overview of the chemical structures of the pyrimidine analogues can be found at the end of this thesis.

Pyrimidine analogues can be considered as prodrugs. They have to enter the tumour cell, where they must undergo extensive intracellular metabolism, before they exert their pharmacological effects. The intracellular metabolism includes the sequential phosphorylation to the so-called nucleotides: the monophosphate, diphosphate and triphosphate form. Only in the form of a triphosphate, the pyrimidine analogue is available for incorporation into DNA or RNA.

It is useful to gain insight into the intracellular pharmacokinetics of the nucleotides of pyrimidine analogues. This provides knowledge about the amount of 'activated drug' that reaches the site of action. Information that could ultimately lead to more substantiated, pharmacology-based, dosage regimens. In addition, the measurement of the intracellular nucleotide concentrations could provide insight into the mechanisms underlying treatment resistance or the differences in treatment responses between patients. 
For cytarabine and gemcitabine, the intracellular pharmacokinetics have been extensively studied over the years [3-20]. However, for the other pyrimidine analogues, our knowledge about their intracellular metabolism is almost entirely based on in vitro studies and animal studies. For 5-FU, azacitidine, decitabine and trifluridine, the intracellular pharmacokinetics in patients treated with these agents were not, or only very minimally investigated [21-25]. This is directly related to the lack of suitable bio-analytical assays.

A number of obstacles play a role here. First, the intracellular concentrations of pyrimidine analogue nucleotides are low, thus highly sensitive measuring equipment is required. Second, the pyrimidine analogue nucleotides are very similar to the physiological pyrimidine nucleotides, thus it is not easy to distinguish the pyrimidine analogue nucleotides from their natural counterparts. These two issues proved to be greater obstacles for the quantification of the nucleotides of 5-FU, azacitidine and decitabine than they had been for the quantification of the nucleotides of cytarabine and gemcitabine.

Thanks to continuous developments in the field of bio-analysis, we are getting increasingly sensitive and selective analytical techniques. The first objective of this thesis was to use the latest analytical techniques to develop suitable bio-analytical assays for the quantification of the intracellular nucleotides of azacitidine and 5-FU in cells of patients that are treated with these agents. The second objective of this thesis was to explore the intracellular pharmacokinetics of the 5-FU nucleotides and the nucleotides of gemcitabine and its deaminated metabolite 2',2'-difluoro-2'-deoxyuridine ( $\mathrm{dFdU}$ ) in patients that were treated with capecitabine and gemcitabine, respectively.

As an introduction to this thesis, Chapter 1 presents a comprehensive overview of the clinical studies that measured the intracellular nucleotide concentrations of pyrimidine analogues in cancer patients. Chapter 2 focuses on azacitidine and decitabine. Chapter 2.1 presents a concise drug review of azacitidine and decitabine, which describes how these pyrimidine analogues were rediscovered in the previous decade due to their DNA demethylating properties. Chapter 2.2 describes the development of a bio-analytical assay for the quantitative determination of azacitidine triphosphate in peripheral blood mononuclear cells. Because azacitidine triphosphate is very similar to the endogenous 
nucleotides uridine triphosphate and the naturally occurring $\left[{ }^{13} \mathrm{C}\right]-$ and $\left[{ }^{15} \mathrm{~N}\right]-$ isotopes of cytidine triphosphate, and azacitidine triphosphate also has almost the same mass as these endogenous nucleotides ( $\Delta<0.02 \mathrm{Da}$ ), highresolution mass spectrometry was required to quantify azacitidine triphosphate in cells. Chapter 3 focuses on the fluoropyrimidines 5-FU and capecitabine. Chapter 3.1 describes the development of an LC-MS/MS assay for quantitative determination of the three intracellular 5-FU nucleotides that are responsible for the antineoplastic effects of 5-FU. In Chapter 3.2 this assay is deployed to explore the intracellular pharmacokinetics of the 5-FU nucleotides in patients treated with capecitabine. In Chapter 4 the intracellular pharmacokinetics of gemcitabine, its deaminated metabolite dFdU and their nucleotides are studied. Finally, in the Summary, Conclusions and Perspectives all results are discussed and put in perspective.

Altogether this thesis presents the development of two sophisticated analytical assays, using the latest bio-analytical techniques, which make it possible for the first time to measure the intracellular concentrations of the pharmacologically active nucleotides of azacitidine and 5-FU, respectively. In addition, the assay for the intracellular quantification of the 5-FU nucleotides and a previously developed assay for the quantification of gemcitabine, dFdU and their nucleotides were deployed to study the intracellular pharmacokinetics of the nucleotides involved in patients treated with capecitabine and gemcitabine, respectively. 


\section{REFERENCES}

[1] European Medicines Agency. https://www. ema.europa.eu/en/medicines. Accessed: August 28, 2019.

[2] CBG-MEB Medicines evaluation board. https://www.geneesmiddeleninformatiebank. nl/en. Accessed: August 28, 2019.

[3] Liliemark JO, Plunkett W, Dixon DO. Relationship of 1-beta-Darabinofuranosylcytosine in plasma to 1 -beta-D-arabinofuranosylcytosine 5'-triphosphate levels in leukemic cells during treatment with high-dose 1-betaD-arabinofuranosylcytosine. Cancer Res 1985;45:5952-7.

[4] Plunkett W, Liliemark JO, Adams $T M$, et al. Saturation of 1-beta-Darabinofuranosylcytosine 5'-triphosphate accumulation in leukemia cells during highdose 1-beta-D-arabinofuranosylcytosine therapy. Cancer Res 1987;47:3005-11.

[5] Gandhi V, Plunkett W, Du M, et al. Prolonged infusion of gemcitabine: Clinical and pharmacodynamic studies during a phase I trial in relapsed acute myelogenous leukemia. J Clin Oncol 2002;20:665-673.

[6] Tempero M, Plunkett W, Ruiz van Haperen VW, et al. Randomized Phase II Comparison of Dose-Intense Gemcitabine: Thirty-Minute Infusion and Fixed Dose Rate Infusion in Patients With Pancreatic Adenocarcinoma. J Clin Oncol 2003;21:3402-3408.

[7] Cattel L, Airoldi M, Delprino L, et al. Pharmacokinetic evaluation of gemcitabine and 2', 2'-difluorodeoxycytidine-5'triphosphate after prolonged infusion in patients affected by different solid tumors. Ann Oncol 2006;17 Suppl 5:v142-7.

[8] Soo RA, Wang LZ, Tham LS, et al. A multicentre randomised phase II study of carboplatin in combination with gemcitabine at standard rate or fixed dose rate infusion in patients with advanced stage non-small-cell lung cancer. Ann Oncol 2006;17:1128-1133.

[9] Nieto Y, Aldaz A, Rifón J, et al. Phase I and Pharmacokinetic Study of Gemcitabine Administered at Fixed-Dose Rate, Combined with Docetaxel/Melphalan/Carboplatin, with Autologous Hematopoietic ProgenitorCell Support, in Patients with Advanced Refractory Tumors. Biol Blood Marrow Transplant 2007;13:1324-1337.
[10] Veltkamp SA, Jansen RS, Callies S, et al. Oral administration of gemcitabine in patients with refractory tumors: a clinical and pharmacologic study. Clin Cancer Res 2008;14:3477-86.

[11] Poplin E, Feng Y, Berlin J, et al. Phase III, Randomized Study of Gemcitabine and Oxaliplatin Versus Gemcitabine (fixed-dose rate infusion) Compared With Gemcitabine (30-minute infusion) in Patients With Pancreatic Carcinoma E6201: A Trial of the Eastern Cooperative Oncology Group. J Clin Oncol 2009;27:3778-3785.

[12] Caffo O, Fallani S, Marangon E, et al. Pharmacokinetic study of gemcitabine, given as prolonged infusion at fixed dose rate, in combination with cisplatin in patients with advanced non-small-cell lung cancer. Cancer Chemother Pharmacol 2010;65:1197-202.

[13] Plunkett W, Liliemark JO, Estey E, et al. Saturation of ara-CTP accumulation during high-dose ara-C therapy: pharmacologic rationale for intermediate-dose ara-C. Semin Oncol 1987;14:159-66.

[14] Estey EH, Keating MJ, McCredie KB, et al. Cellular ara-CTP pharmacokinetics, response, and karyotype in newly diagnosed acute myelogenous leukemia. Leukemia 1990;4:959.

[15] Abbruzzese JL, Grunewald R, Weeks EA, et al. A phase I clinical, plasma, and cellular pharmacology study of gemcitabine. J Clin Oncol 1991;9:491-8.

[16] Grunewald R, Kantarjian H, Du M, et al. Gemcitabine in leukemia: a phase I clinical, plasma, and cellular pharmacology study. $J$ Clin Oncol 1992;10:406-13.

[17] Gandhi V, Xu YZ, Estey E. Accumulation of arabinosyluracil 5'-triphosphate during arabinosylcytosine therapy in circulating blasts of patients with acute myelogenous leukemia. Clin Cancer Res 1998;4:1719-26.

[18] Kroep JR, Giaccone G, Voorn DA, et al. Gemcitabine and paclitaxel: pharmacokinetic and pharmacodynamic interactions in patients with non-small-cell lung cancer. J Clin Oncol 1999;17:2190-7. 
[19] van Moorsel CJ, Kroep JR, Pinedo HM, et al. Pharmacokinetic schedule finding study of the combination of gemcitabine and cisplatin in patients with solid tumors. Ann Oncol 1999;10:441-448.

[20] Patel SR, Gandhi V, Jenkins J, et al. Phase II clinical investigation of gemcitabine in advanced soft tissue sarcomas and window evaluation of dose rate on gemcitabine triphosphate accumulation. J Clin Oncol 2001;19:3483-9.

[21] Spears CP, Gustavsson BG, Mitchell MS, et al. Thymidylate synthetase inhibition in malignant tumors and normal liver of patients given intravenous 5-fluorouracil. Cancer Res 1984;44:4144-50.

[22] Spears CP, Gustavsson BG, Berne M, et al. Mechanisms of innate resistance to thymidylate synthase inhibition after 5-fluorouracil. Cancer Res 1988;48:58945900.

[23] Peters GJ, Lankelma J, Kok RM, et al. Prolonged retention of high concentrations of 5 -fluorouracil in human and murine tumors as compared with plasma. Cancer Chemother Pharmacol 1993;31:269-76.

[24] Jansen RS, Rosing H, Wijermans PW, et al. Decitabine triphosphate levels in peripheral blood mononuclear cells from patients receiving prolonged low-dose decitabine administration: A pilot study. Cancer Chemother Pharmacol 2012;69:1457-1466.

[25] Wang $\mathrm{H}$, Chen P, Wang J, et al. In vivo quantification of active decitabinetriphosphate metabolite: a novel pharmacoanalytical endpoint for optimization of hypomethylating therapy in acute myeloid leukemia. AAPS J 2013;15:242-9. 


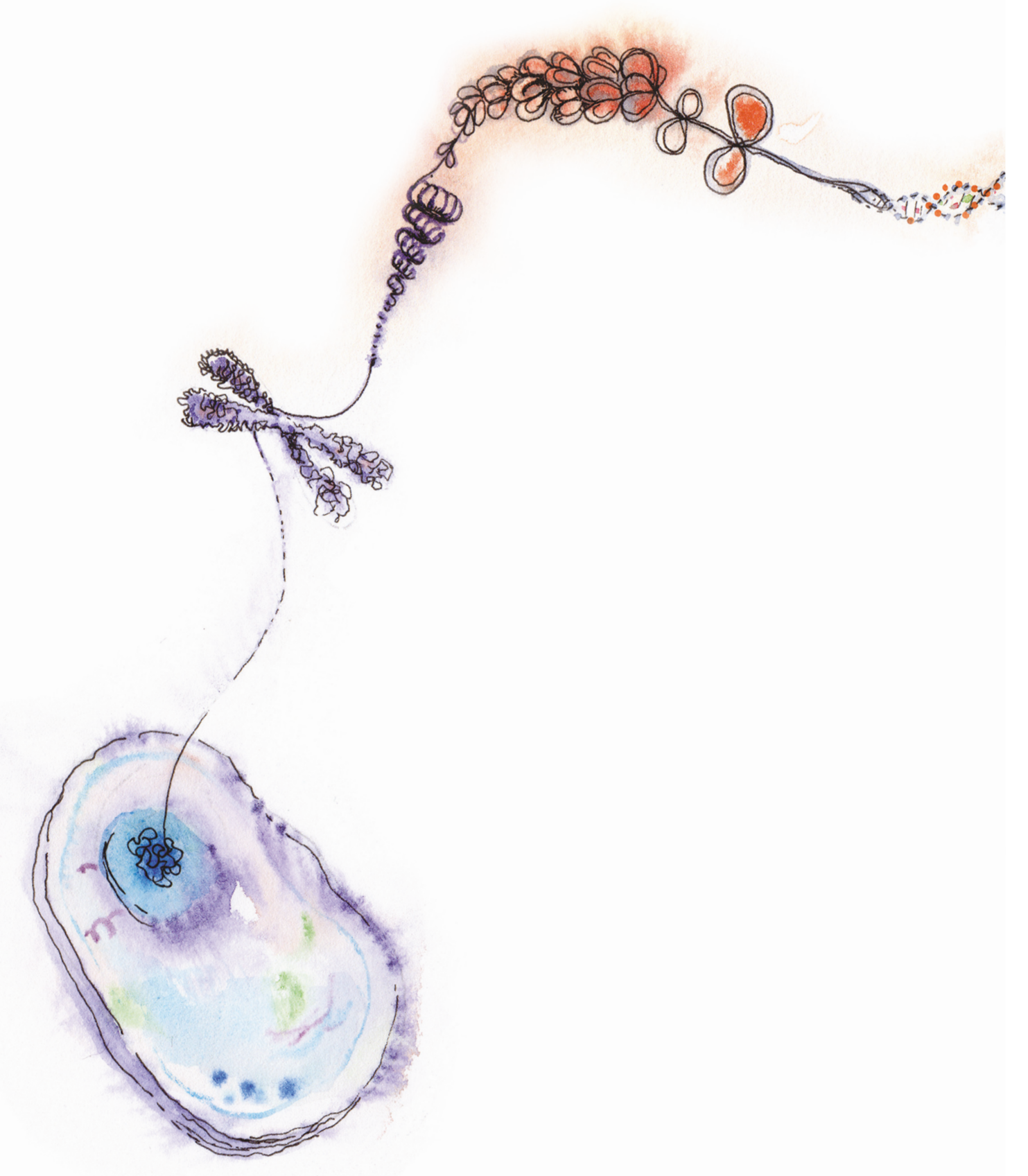


मों

in

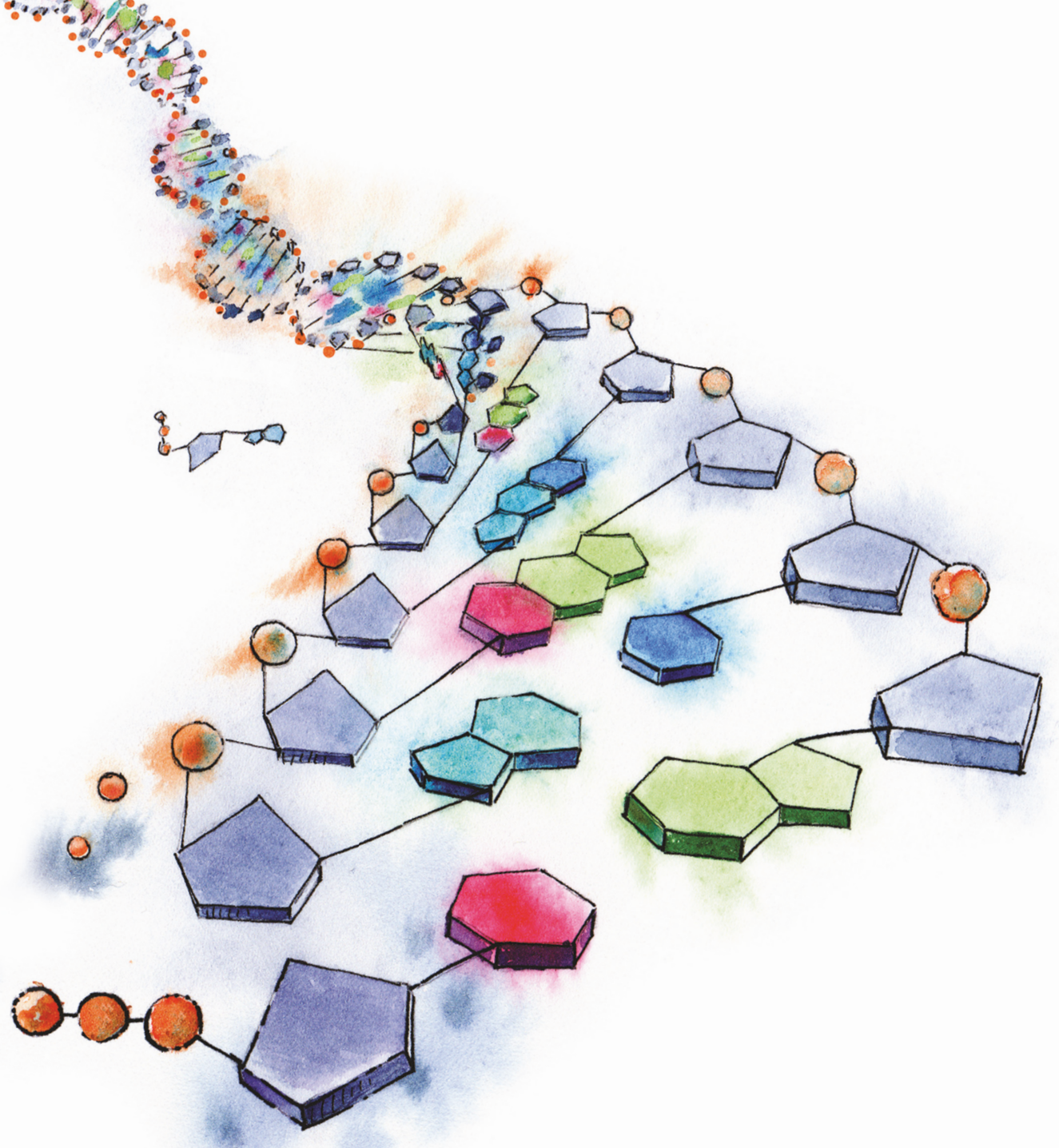

CHAPTER 1 


\section{CHAPTER 1}

Intracellular Pharmacokinetics of Pyrimidine Analogues used in Oncology and the Correlation with Drug Action

- Review Article -

Submitted for Publication

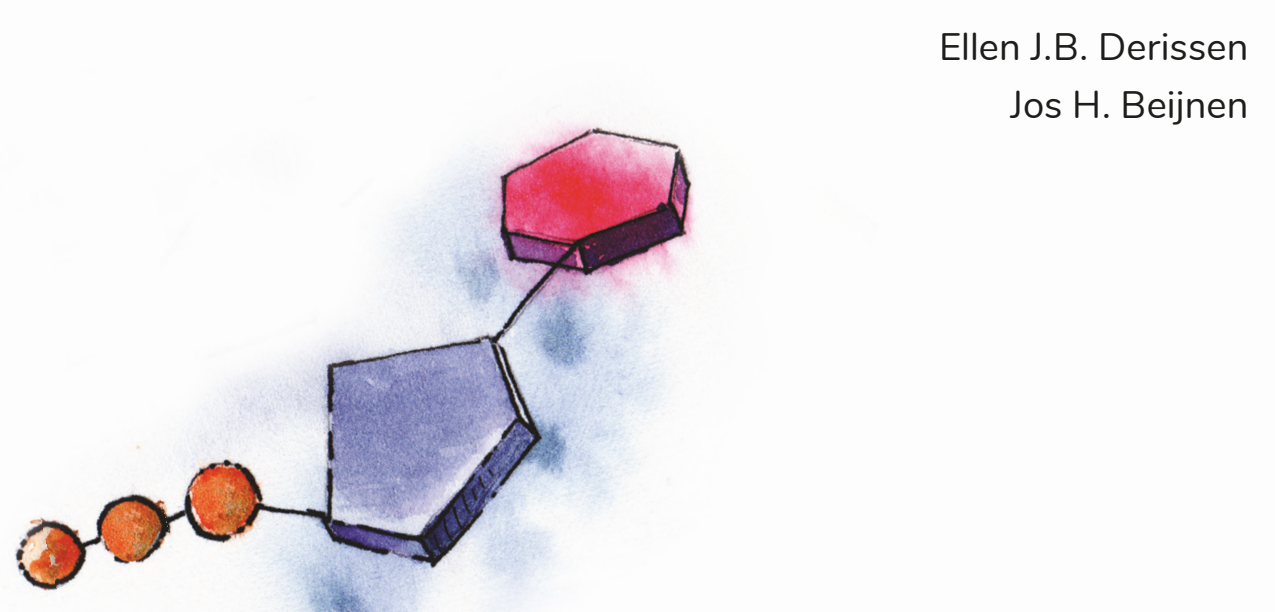




\section{CONTENTS}

\section{Introduction}

1. 5-Fluorouracil and Capecitabine

1.1. Plasma PK of 5-FU and capecitabine

1.2. Intracellular metabolism and mechanism of action

1.3. Clinical studies investigating the intracellular 5-FU nucleotide concentrations

1.4. 5-Fluorouracil and capecitabine in conclusion

2.

\section{Cytarabine}

2.1. Plasma PK of cytarabine

2.2. Intracellular metabolism and mechanism of action

2.3. Clinical studies investigating the intracellular cytarabine nucleotide concentrations

2.3.1. Relationship between cytarabine dose, plasma concentrations and intracellular ara-CTP concentrations

2.3.2. Saturation of the intracellular accumulation of ara-CTP

2.3.3. Optimization of dosing regimens in relation to the intracellular accumulation of ara-CTP

2.3.4. Relationship between intracellular ara-CTP concentrations and efficacy

2.3.5. The role of arabinosyluracil triphosphate (ara-UTP) during cytarabine therapy

2.4. Cytarabine in conclusion 


\section{Gemcitabine}

3.1. Plasma PK of gemcitabine

3.2. Intracellular metabolism and mechanism of action

3.3. Intracellular PK of gemcitabine - Important lessons from preclinical studies

3.4. Clinical studies investigating the intracellular gemcitabine nucleotide concentrations

3.4.1. Saturation of the intracellular accumulation of dFdCTP

3.4.2. Increasing the intracellular accumulation of dFdCTP by prolonging the infusion time

3.4.3. Relationship between intracellular dFdCTP concentrations and efficacy

3.4.4. Relationship between intracellular dFdCTP concentrations and toxicity

3.4.5. Interpretation and future perspectives

3.4.6. The role of $d F d U$ nucleotides during gemcitabine therapy

3.4.7. Combination regimens and intracellular PK interactions

3.5. Gemcitabine in conclusion

4. Azacitidine and Decitabine

4.1. Plasma PK of azacitidine and decitabine

4.2. Intracellular metabolism and mechanism of action

4.3. Clinical studies investigating the intracellular azacitidine and decitabine nucleotide concentrations

4.4. Azacitidine and decitabine in conclusion

\section{Discussion}

Keywords: intracellular pharmacokinetics; pyrimidine analogues; nucleotides; 5-fluorouracil; capecitabine; cytarabine; gemcitabine; azacitidine; decitabine. 


\section{INTRODUCTION}

Pyrimidine analogues form a classical group of cytostatic drugs that still have a very important place in the treatment of both solid and hematologic malignancies. Pyrimidine analogues are structural analogues of one of the physiological pyrimidine nucleobases: cytosine, uracil or thymine or of their respective nucleosides: (deoxy)cytidine, uridine and thymidine (Fig. 1).

The first important pyrimidine analogue, 5-fluorouracil (5-FU), was synthesized in 1957 and still has an important place in the treatment of various solid tumours including colorectal and breast cancer. After 5-FU, several other pyrimidine analogues followed. In 1969, cytarabine was introduced for the treatment of acute leukaemia. Today, cytarabine is still one of the cornerstones in the treatment of acute myeloid leukaemia (AML). Moreover, it has a place in the treatment of various other haematological malignancies. The success of cytarabine as an anti-leukemic drug stimulated the research for other cytidine analogues that had anti-tumour activity and led to the first preclinical studies with azacitidine and decitabine in the 1960s [1]. Although azacitidine and decitabine initially seemed promising, the first clinical trials were not very impressive, limiting enthusiasm for these drugs as anti-tumour agents [2-4]. In 1995, gemcitabine was introduced. Although the structure of gemcitabine was similar to that of cytarabine, it had a different spectrum of anti-tumour activity [5]. Unlike cytarabine, gemcitabine exhibited activity in solid tumours. Today, gemcitabine has an important place in the treatment of non-small cell lung cancer, pancreatic cancer, bladder cancer and ovarian cancer. In the 1990s, capecitabine was developed as a more convenient oral alternative to intravenous 5-FU therapy. As a prodrug of 5-FU, capecitabine was rationally designed to mimic continuous infusion of 5-FU with a certain degree of tumour selectivity [6]. In this decade, there was also renewed interest in azacitidine and decitabine. This time because of their DNA demethylating properties in patients with myelodysplastic syndrome (MDS) [7-10]. Today, azacitidine and decitabine have gained their place in the treatment of MDS and AML [11].

Pyrimidine analogues can be considered as prodrugs, like their natural counterparts, they have to be activated within the cell. The intracellular activation involves several metabolic steps among which sequential phosphorylation of the 


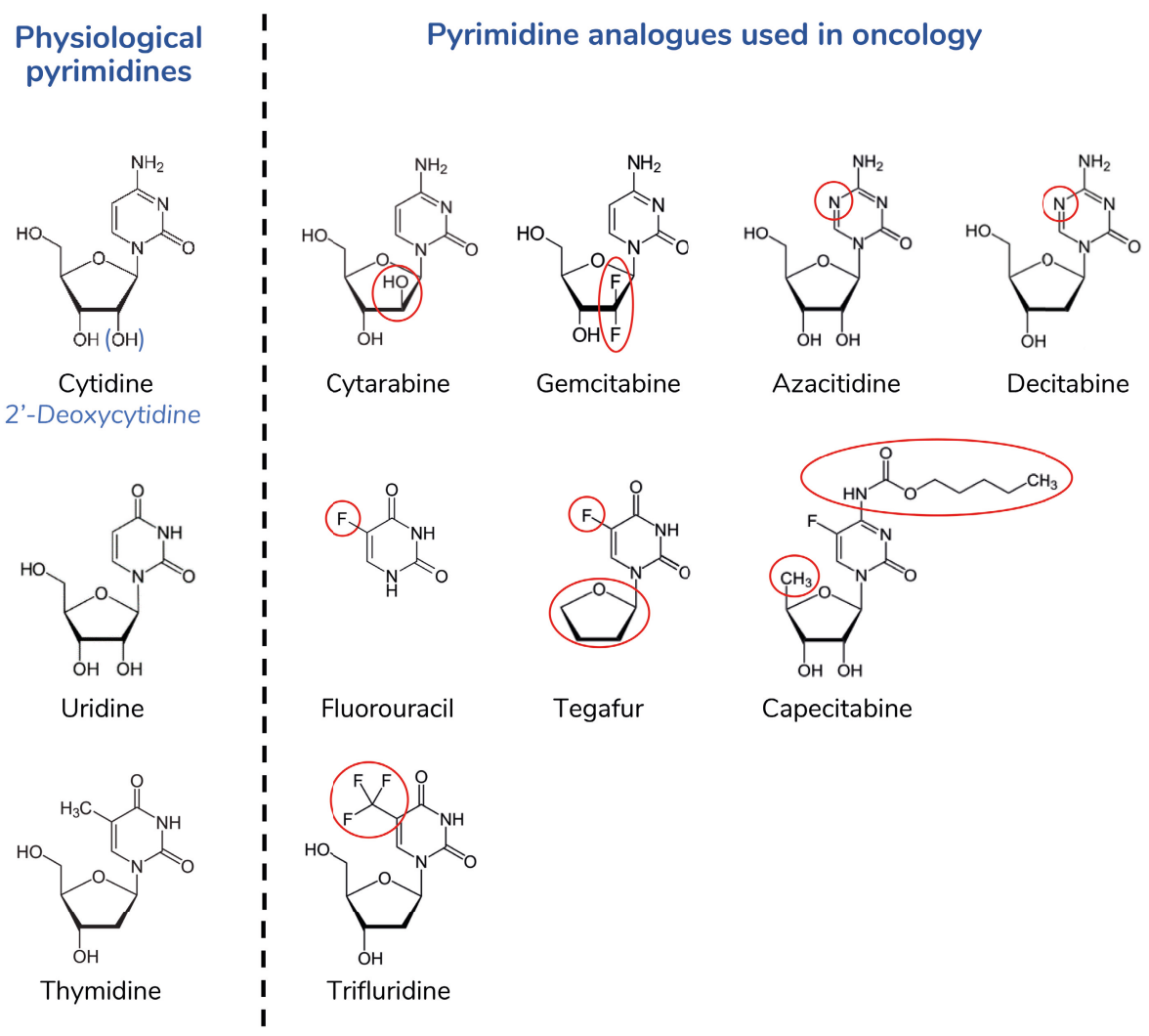

Figure 1. Chemical structures of the physiological pyrimidines (left) and the pyrimidine analogues used as anticancer drugs (right).

pyrimidine analogue to its monophosphate, diphosphate and triphosphate (Fig. 2). Ultimately, these intracellularly phosphorylated anabolites, called nucleotides, are responsible for the pharmacological effects of the pyrimidine analogue. For instance, as the triphosphate form can compete with its natural counterpart for incorporation into the DNA or RNA, thereby interfering with the DNA or RNA synthesis and with their normal functions. For 5-FU, 5-fluorodeoxyuridine monophosphate is an important anabolite, as it inhibits the enzyme thymidylate synthase.

Since the intracellularly formed nucleotides are ultimately responsible for the effect of the pyrimidine anticancer drugs, it would be useful to understand the intracellular pharmacokinetics (PK) of these nucleotides during treatment. 
In this review, we summarize the findings of more than 30 clinical studies that measured the intracellular concentrations of pyrimidine nucleotides in cancer patients. These studies were intended to get insight into the intracellular PK of the pyrimidine analogues. A number of studies was also aimed at finding dose schedules that would maximize the intracellular triphosphate concentrations in order to optimize the anticancer effect of the drug. Although, on a pharmacological basis, the accumulation and retention of nucleotides appears critical to the cytotoxicity of a pyrimidine drug, only a few studies have actually investigated the relationship between the intracellular nucleotide concentrations measured in cancer patients and the anti-tumour effect or the occurrence of toxicities.

The objective of this review was to gain more insight into the parallels between the different pyrimidine analogues with regard to their intracellular pharmacokinetics. In this way, we can learn the most from what is already known about the intracellular PK of the other drugs in this group. This information could ultimately be very useful to optimize current treatment regimens.

In this review, we included only clinical studies in which intracellular nucleotide concentrations were measured in cancer patients during and after the treatment with a pyrimidine analogue. Although much of our knowledge about the intracellular pharmacology of the pyrimidine analogues comes from in vitro and animal studies, these studies were deliberately not included in this review as it remains difficult to extrapolate these results to the clinical situation. However, knowledge derived from preclinical studies, which was considered relevant for the interpretation of the clinical studies, was briefly summarized at the beginning of each chapter.

This review consists of four chapters, in which the six pyrimidine analogues are discussed that are currently considered the most important. Chapter 1: 5-Fluorouracil and Capecitabine. Chapter 2: Cytarabine. Chapter 3: Gemcitabine and Chapter 4: Azacitidine and Decitabine. Each chapter begins with a brief summary of the plasma PK of the pyrimidine analogue in question. This is followed by a description of the intracellular metabolism and the mechanisms of action. Then, where deemed useful, essential lessons about the intracellular PK obtained from preclinical studies are summarized. In the body of each chapter, 


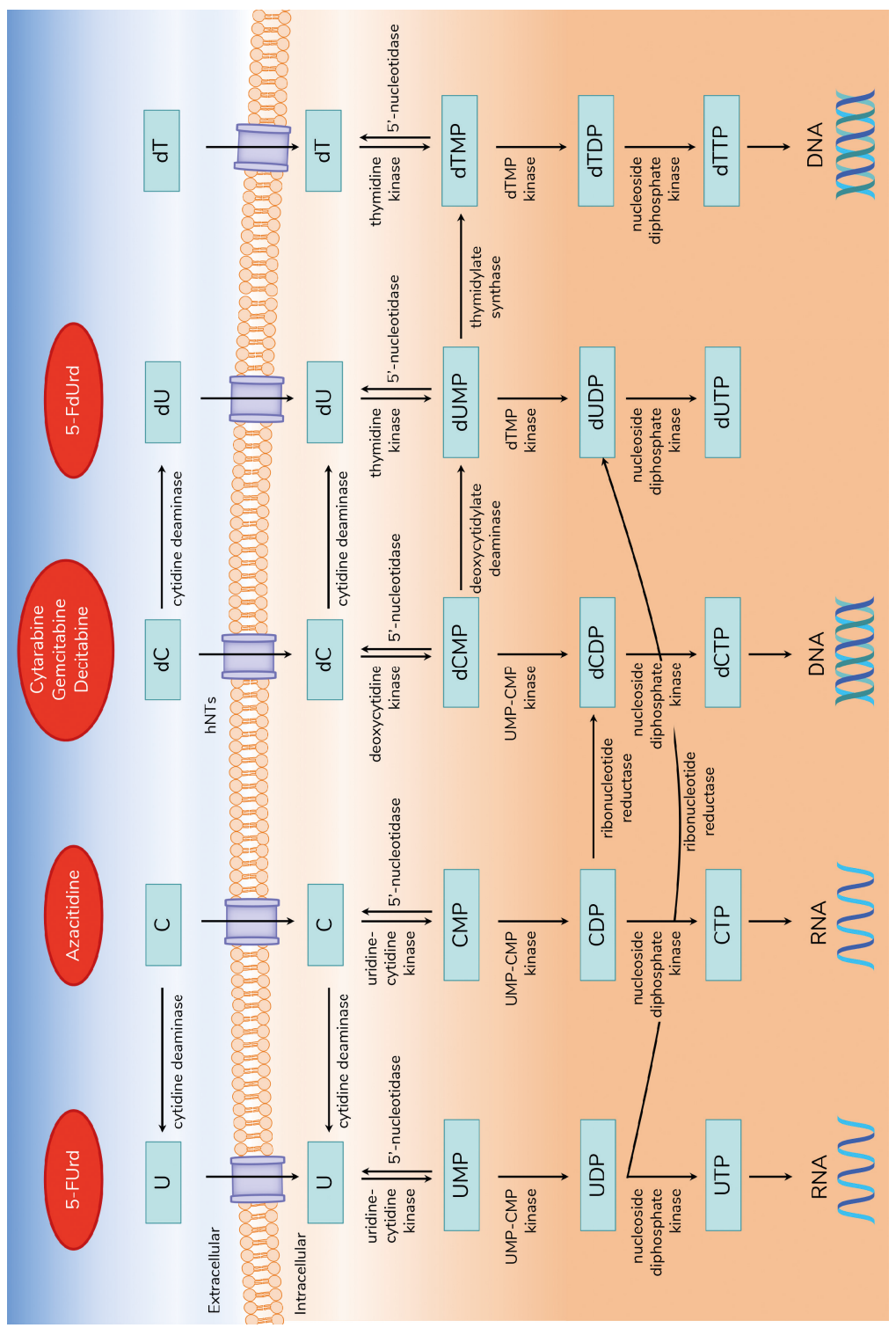

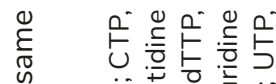

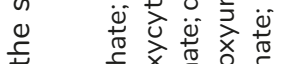

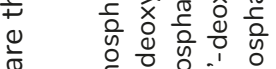

$\frac{\frac{1}{n}}{\frac{1}{n}}$

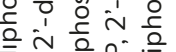

文

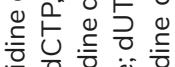

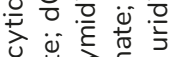

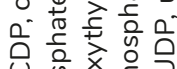

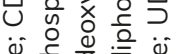

to

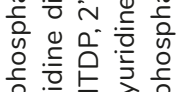

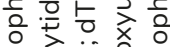
ปे $\varepsilon$ X 응 흥

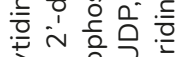
ปิํํ응

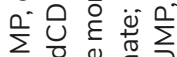
U

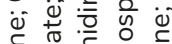

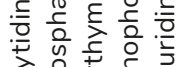

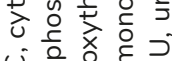
$\cup$ 웅 孚 旁是

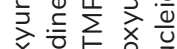

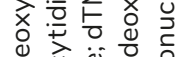
论过 기을

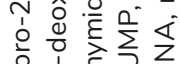

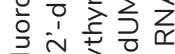
L เ

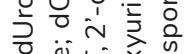

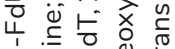
ᄂ बं 흐 $\vec{x} \cdot \frac{u}{0} \bar{y}^{\circ}$ 亏웡 ㅎำ

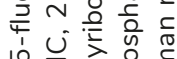

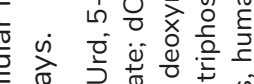
ते

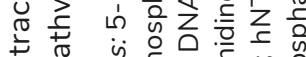
吾 i.

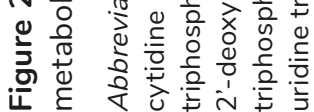


all clinical studies that examined the intracellular PK of the pyrimidine analogue in question are discussed. The results of this review are also summarized in Tables 1 and 2.

\section{5-FLUOROURACIL AND CAPECITABINE}

The first important pyrimidine analogue, 5-fluorouracil (5-FU), was synthesized in 1957. 5-FU was developed following the observation that radiolabelled uracil was more readily incorporated into rat hepatomas than into healthy liver tissue $[12,13]$. Presuming the preferential incorporation of uracil in tumour tissue, 5-FU was rationally designed. The hydrogen atom at the C-5 position of uracil was replaced by fluorine (Fig. 1). This specific substitution was selected as it might inhibit the conversion of deoxyuridine nucleotides to thymidine nucleotides, by preventing the addition of a methyl group at the C-5 position. Therefore, the replacement of hydrogen in this position was thought to have a good chance of inhibiting DNA synthesis and, thus, growth. The selection of fluorine to replace the hydrogen in uracil was based on their similar van der Waals radii [14].

Today, 5-FU still has an important place in the treatment of a range of cancer types, including those originating into the gastrointestinal tract, breast and head and neck region. Moreover, after 5-FU, several other fluoropyrimidines have been investigated. In the 1990 s capecitabine was developed as an oral alternative to intravenous 5-FU therapy. As a prodrug of 5-FU, capecitabine was rationally designed to mimic continuous infusion of 5-FU and deliver 5-FU preferentially to tumour tissue [6]. Following absorption, capecitabine is rapidly converted to 5-FU via a three-step enzymatic cascade. 5-FU is generated preferentially in tumour by exploiting the higher activity of the enzyme thymidine phosphorylase in tumour tissue compared with normal tissue [15]. Like 5-FU, capecitabine is still a widely used cytostatic agent today, which has an important place in the treatment of several malignancies, including colorectal, gastric, pancreatic, breast and head and neck cancer. 


\section{Plasma PK of 5-FU and capecitabine}

Many different schedules have been used for the intravenous administration of 5-FU. After administration, 5-FU is rapidly eliminated from the plasma with an elimination half-life $\left(t_{1 / 2}\right)$ of only 5-20 min [16]. Approximately $80 \%$ of $5-F U$ is inactivated in the liver by the enzyme dihydropyrimidine dehydrogenase (DPD) [16]. DPD is the rate-limiting enzyme in 5-FU catabolism and converts 5-FU to the inactive metabolite dihydrofluorouracil. Dihydrofluorouracil is further degraded to a-fluoroureido propionic acid and the last catabolite a-fluoro- $\beta$ alanine $[17,18]$. This means that only a small fraction of the administered $5-F U$ is available for intracellular anabolism to the active nucleotides.

Large inter-individual differences in the clearance of 5-FU have been found [19]. The DPD activity is shown to be highly variable between individuals [20-22]. Population studies demonstrated that the DPD activity exhibited a Gaussian distribution, in which the high- and low-end activities varied by a factor 10 [20$22]$. Approximately $3-5 \%$ of the population is partially DPD deficient $[20,21]$.

The clearance of 5-FU by DPD was found to be saturable [23]. At higher 5-FU doses, the relative clearance decreases. Therefore, plasma concentrations do not only depend on the dose, but also on the administration rate. Continuous infusion of 5-FU leads to a more efficient plasma clearance than bolus injections and, therefore, permits the administration of higher total 5-FU doses [23]. Furthermore, it is assumed that by continuous administration of 5-FU more cells are reached during the S-phase of the cell cycle $[23,24]$. Indeed, continuous infusion was shown to be more effective than bolus administration of 5-FU [25]. Also the toxicity pattern of 5-FU was found to be dependent on the velocity of administration. An intravenous bolus typically causes myelotoxicity, whereas continuous intravenous infusions often lead to more severe stomatitis and handfoot syndrome $[25,26]$.

Capecitabine was rationally designed to mimic continuous infusion of 5-FU $[23,24]$. After oral administration, capecitabine is rapidly and almost completely absorbed from the gastrointestinal tract. Subsequently, it is converted into 5-FU through a series of three metabolic steps. First, capecitabine is converted to 5'-deoxy-5-fluorocytidine (5'-dFCR) by carboxylesterase, an enzyme located primarily in the liver. 5 '-dFCR is then converted to 5'-deoxy-5-fluorouridine 
(5'-dFUR) by cytidine deaminase, which is principally located in the liver and in tumour tissue. In the third step, 5'-dFUR is converted to 5-FU by thymidine phosphorylase. This enzyme is present at higher concentrations in solid tumour tissue than in normal tissues $[27,28]$. Therefore, the third activation step preferentially takes place in tumour tissue rather than normal tissue, which confers a degree of tumour specificity to capecitabine [15,27-29].

The recommended dose of capecitabine is $1000-1250 \mathrm{mg} / \mathrm{m}^{2}$ twice daily for two weeks, followed by a 1-week rest period, given in 3 week cycles. It is recommended to take capecitabine at the end of a meal. Food intake delays and reduces the absorption [15]. Following the administration of capecitabine, maximum plasma concentrations $\left(\mathrm{C}_{\max }\right)$ of both capecitabine and 5-FU were reached after $2 \mathrm{~h}$, indicating that capecitabine is very rapidly metabolized to 5-FU [15]. The 5-FU plasma concentrations reached after capecitabine administration were considerably lower than those after therapeutic doses of intravenous 5-FU [15]. The $\mathrm{C}_{\max }$ and AUC of capecitabine were proportional to the administered capecitabine dose. However, for 5-FU, dose proportionality was only present up to capecitabine doses of $829 \mathrm{mg} / \mathrm{m}^{2}$. At higher doses, the increase in AUC of 5-FU was larger than proportional due to saturation of DPD $[15,30]$. Capecitabine has a short $t_{1 / 2}$ of approximately $50 \mathrm{~min}$. The apparent $t_{1 / 2}$ of $5-F U$, after capecitabine administration, was similar to that of the parent compound, as the elimination of 5-FU was rate-limited by its formation [15]. The interpatient variability of the pharmacokinetic parameters of capecitabine and 5-FU was high due to variability in the activity of the enzymes involved in the capecitabine metabolism [15].

\section{Intracellular metabolism and mechanism of action}

5-FU rapidly enters the cell using the same transport mechanism as uracil $[16,31]$. Once in the cell, $5-\mathrm{FU}$ has to be activated by ribosylation and sequential phosphorylation. The activation of 5-FU to its nucleotides can proceed via three pathways [32,33]. 1.) 5-FU can be converted directly to 5-fluorouridine monophosphate (FUMP) by the enzyme orotate phosphoribosyl transferase. 2.) Alternatively, 5-FU can be converted to FUMP in two steps, via 5-fluorouridine, through the sequential actions of uridine phosphorylase and uridine kinase. FUMP is further phosphorylated to 5-fluorouridine diphosphate (FUDP) 
and the active metabolite 5-fluorouridine triphosphate (FUTP) by uridine monophosphate kinase and uridine diphosphate kinase, respectively. FUDP might also be converted to 5-fluoro-2'-deoxyuridine diphosphate (FdUDP) by ribonucleotide reductase. 3.) The third activation pathway involves the conversion of 5-FU to 5-fluoro-2'-deoxyuridine by thymidine phosphorylase. 5-fluoro-2'-deoxyuridine can then be phosphorylated to the active metabolite 5-fluoro-2'-deoxyuridine monophosphate (FdUMP) by thymidine kinase. FdUMP can also be further phosphorylated to FdUDP and the third active metabolite 5-fluoro-2'-deoxyuridine triphosphate (FdUTP). These reactions are catalysed by uridine monophosphate kinase and uridine diphosphate kinase, respectively. Conversely, the nucleotides may also be degraded to their inactive nucleosides by 5 '-nucleotidases and phosphatases [32,33].

Ultimately, three intracellularly formed metabolites (nucleotides) are held responsible for the antineoplastic effects of 5-FU. These are FUTP, FdUTP, and FdUMP. FUTP is incorporated into RNA and interferes with normal RNA processing and function. FdUTP is incorporated into DNA, leading to DNA damage and ultimately cell death. FdUMP inhibits thymidylate synthase, the enzyme that catalyses the methylation of deoxyuridine monophosphate (dUMP) to deoxythymidine monophosphate (dTMP). Inhibition of thymidylate synthase by FdUMP leads to accumulation of deoxyuridine triphosphate (dUTP) and depletion of deoxythymidine triphosphate (dTTP). This imbalance has deleterious consequences for DNA synthesis and repair, ultimately leading to cell death $[24,34,35]$.

\section{Clinical studies investigating the intracellular 5-FU nucleotide concentrations}

Most of our knowledge about the intracellular metabolism of 5-FU and its mechanisms of action was derived from in vitro experiments and animal studies [36-39]. Little is known about the formation of 5-FU nucleotides in patients that are treated with 5-FU or capecitabine. In the 1980s and 1990s, several studies investigated the intracellular FdUMP concentrations in patients that were treated with an intravenous 5-FU bolus [40-42]. 
Spears et al. determined the intracellular FdUMP concentrations in tumour biopsies obtained from 21 patients with cancer, mainly originating from the gastrointestinal tract, who had previously received an intravenous 5-FU bolus of $500 \mathrm{mg} / \mathrm{m}^{2}$ [40]. Intraoperative biopsy specimens were obtained 20 min to $4 \mathrm{~h}$ after 5-FU administration. In 9 patients, synchronous biopsies of normal liver tissue were obtained along with samples of hepatic metastases. For each patient, biopsies were obtained at a single time point after 5-FU administration. However, if the measured FdUMP levels for the total group of patients were expressed against the time interval between 5-FU administration and tissue biopsy, a graph was obtained that could be interpreted as a pharmacokinetic profile. FdUMP levels were highest (up to $1000 \mathrm{pmol} / \mathrm{g}$ ) at the earliest time interval studied, 20-30 min after 5-FU administration, indicating that 5-FU is rapidly converted to FdUMP. Subsequently, FdUMP levels appeared to decrease exponentially through $120 \mathrm{~min}$. For the time points beyond $120 \mathrm{~min}$, greater scatter was found. The FdUMP levels measured in gastro-intestinal tumours were higher than those found in normal liver tissue (mean \pm SD; $238 \pm 559$ pmol/g vs. $35 \pm 45 \mathrm{pmol} / \mathrm{g}$ ) [40].

In a subsequent study, Spears et al. obtained surgical tumour biopsies from 54 patients with metastatic adenocarcinoma who had received an intravenous $5-\mathrm{FU}$ bolus of $500 \mathrm{mg} / \mathrm{m}^{2}$ [41]. Biopsies were obtained 20-400 min after administration of the 5-FU. The intracellular FdUMP and dUMP concentrations were measured and expressed against the observed percentages of thymidylate synthase inhibition. This suggested that a minimum FdUMP concentration of $75 \mathrm{pmol} / \mathrm{g}$ and a minimal FdUMP/dUMP ratio of 0.001 was needed to achieve maximal TS inhibition [41].

Peters et al. measured the 5-FU and FdUMP concentrations in liver and colon biopsy specimens (of both healthy and tumour tissue) obtained from 39 patients after they had received an intravenous 5-FU bolus of $500 \mathrm{mg} / \mathrm{m}^{2}$ [42]. Although most of the 5-FU was rapidly eliminated from the plasma, very low 5-FU plasma concentrations, ranging from 0.01 to $1 \mu \mathrm{M}$, could be measured for several days after the 5-FU bolus. However, the 5-FU concentrations measured in tumour tissue were substantially higher. Between 4 to $48 \mathrm{~h}$ after administration, the 5-FU tissue-to-plasma concentration ratio was at least 10. The highest intracellular FdUMP concentrations were observed shortly 
after 5-FU administration. 1-5 $\mathrm{h}$ after 5-FU administration, the intracellular FdUMP concentrations measured in tumour tissue ranged from 10 to 1700 $\mathrm{pmol} / \mathrm{g}$. FdUMP remained present in the cell for a long time. 19-27 h after 5-FU administration, FdUMP concentrations were below $200 \mathrm{pmol} / \mathrm{g}$. After $48 \mathrm{~h}, \mathrm{FdUMP}$ was measurable only in a few samples. The concentrations of FdUMP measured in tissue samples obtained the same time points after the 5-FU bolus showed large inter-individual variation. Moreover, this study confirmed that the FdUMP concentrations in tumour tissue were higher than those observed in healthy tissue from the same patient [42].

In the above studies, the intracellular FdUMP concentrations were determined using a competitive-binding assay with bacterial thymidylate synthase as a binding protein. The quantification of FdUMP was based on the displacement of a known amount of radiolabelled $\left[6-{ }^{3} \mathrm{H}\right]-\mathrm{FdUMP}$, measured by scintillation counting [40-43].

Although this "isotope-dilution" assay for the quantification of FdUMP was available since 1979, a sufficiently sensitive assay for the quantification of the other two active nucleotides, FUTP and FdUTP, in cells of patients that were treated with 5-FU was lacking for a long time. Therefore, we developed an ultrasensitive liquid chromatography-tandem mass spectrometry (LC-MS/ MS) assay for simultaneous quantification of the three pharmacologically active 5-FU nucleotides FUTP, FdUTP and FdUMP in peripheral blood mononuclear cells (PBMCs) [44]. The advent of this assay made it possible to get insight into the intracellular 5-FU nucleotide concentrations in samples of patients who were treated with 5-FU or capecitabine.

As a start, the intracellular 5-FU nucleotide concentrations were measured in PBMC samples of five patients that were treated with an intravenous 5-FU bolus of $400 \mathrm{mg} / \mathrm{m}^{2}$ infused over $30 \mathrm{~min}$. Thirty minutes after the end of the infusion, FUTP concentrations between 4.7 and $11 \mu \mathrm{M}$ were measured. FdUMP concentrations were much lower and ranged from 0.02 to $0.6 \mu \mathrm{M}$. The FdUTP concentrations were below the detection limits of the assay [44].

In a subsequent study, we explored the intracellular pharmacokinetics of the three active 5-FU nucleotides during capecitabine treatment [45]. Only 
FUTP could be measured in the PBMC samples (Table 2). Both the FdUTP and FdUMP concentrations were below the detection limits of the assay. No significant correlation was found between the plasma 5-FU exposure and the intracellular FUTP exposure. The FUTP concentration-time profiles demonstrated considerable inter-individual variation. The study also showed that FUTP remained present in the cell for a long time. Twenty-four hours after a single capecitabine dose of 1000 mg, intracellular FUTP concentrations were on average still $41 \%$ of the maximum concentrations found. In accordance with this finding, after 14 days of treatment with capecitabine $\left(850 \mathrm{mg} / \mathrm{m}^{2}\right.$ twice daily), accumulation of FUTP was demonstrated in PBMCs. The area under the FUTP concentration-time curve was significantly increased on day 14 of the treatment compared to day 1 . Remarkably, the intracellular FUTP concentrations measured on day 14 of capecitabine treatment were in the same range (0.6-14 $\mu \mathrm{M})$ as the concentrations observed $30 \mathrm{~min}$. after an intravenous 5 -FU bolus (400 mg/m² in $30 \mathrm{~min}$ ) [45].

As usual in these studies, PBMCs were selected as a surrogate matrix to measure the intracellular nucleotide concentrations. It is important to realize that, by measuring in PBMCs, the tumour-specific activation of capecitabine is not taken into account and that the concentrations in tumour cells might be different.

So far, to the best of our knowledge, it has not been possible to measure FdUTP in cells of patients that were treated with 5-FU or capecitabine. Perhaps an even more sensitive assay is needed to measure these FdUTP concentrations.

\section{5-Fluorouracil and capecitabine in conclusion}

Most of our knowledge about the intracellular metabolism of 5-FU originates from in vitro experiments and animal studies. Little is known about the formation of 5-FU nucleotides in patients that are treated with 5-FU or capecitabine. In the 1980s and 1990s, several studies determined the intracellular FdUMP concentrations in healthy liver and tumour tissue biopsies obtained from patients that were treated with an intravenous 5-FU bolus [40-42]. These studies demonstrated that 5-FU plasma concentrations do not reflect intracellular FdUMP concentrations. Although 5-FU is rapidly eliminated from 
plasma, FdUMP is retained considerably longer in cells and was still measurable 19-27 h after 5-FU administration in most patients [42]. The intracellular FdUMP concentrations showed high inter-individual variation. The FdUMP concentrations in tumour tissue were higher than those observed in healthy tissue from the same patient $[40,42]$. These studies determined the intracellular FdUMP concentrations using a $\left[{ }^{3} \mathrm{H}\right]-$ FdUMP isotope dilution assay with bacterial thymidylate synthase as a binding protein [43].

Until 2015, there was no sufficiently sensitive assay for the quantification of the other two active nucleotides, FUTP and FdUTP, in patient samples. This prompted us to develop an LC-MS/MS assay for the quantification of FdUMP, FdUTP and FUTP in PBMCs and to explore the intracellular pharmacokinetics of these nucleotides during capecitabine treatment $[44,45]$. After treatment with capecitabine, only FUTP could be measured in PBMCs. Apparently, capecitabine is mainly converted into FUTP and to a lesser extent into FdUTP and FdUMP. No significant correlation was found between the plasma 5-FU exposure and the intracellular FUTP exposure. The FUTP concentration-time profiles demonstrated considerable inter-individual variation. FUTP remained much longer present in PBMCs than 5-FU in plasma. During 14 days of treatment with capecitabine twice daily, intracellular accumulation of FUTP was found in PBMCs. 


\section{CYTARABINE}

Cytarabine has been used as a chemotherapeutic agent since 1969 and nowadays has an important place in the treatment of acute myeloid leukaemia ( $A M L)$, acute lymphocytic leukaemia (ALL), chronic myelogenous leukaemia (CML) and non-Hodgkin's lymphoma. Cytarabine (1- $\beta$-Darabinofuranosylcytosine, ara-C) is a deoxycytidine analogue as it combines a cytosine base with an arabinose sugar. The difference with respect to cytidine is that the 2' hydroxyl group of cytarabine is oriented in the trans position, resulting in metabolism similar to that of a deoxyribose nucleoside.

\section{Plasma PK of cytarabine}

After administration, cytarabine is rapidly converted to $1-\beta-D$ arabinofuranosyluracil (ara-U) by the enzyme cytidine deaminase. Cytidine deaminase is ubiquitous in the human body and catalyses the hydrolytic deamination of (deoxy)cytidine to (deoxy)uridine (Fig. 2). Cytidine deaminase is present in plasma, but also in the liver and kidneys, where high levels are found [46].

Over the years, cytarabine has been used in many different dosing schedules. After high-dose cytarabine $\left(3000 \mathrm{mg} / \mathrm{m}^{2}\right.$ infused intravenously in $3 \mathrm{~h}$ ), maximum plasma concentrations of cytarabine were reached within $1 \mathrm{~h}$ and were maintained steadily throughout the infusion [47]. After the end of the infusion, the cytarabine concentration declined rapidly. The elimination pattern is biphasic, with an initial plasma half-life $\left(t_{1 / 2 a}\right)$ of 7 to 20 minutes and a terminal half-life $\left(t_{1 / 2 \beta}\right)$ of 2 to 3 hours $[47,48]$.

Plasma ara- $U$ concentrations generally peaked 0.5 to $2 \mathrm{~h}$ after the end of the infusion and were 5 to 30 times higher than the peak concentrations of cytarabine $[47,49,50]$. Plasma ara- $U$ was rapidly cleared. The $t_{1 / 2}$ value for ara- $U$ elimination from plasma was about $6 \mathrm{~h}$ [47]. Ara- $U$ is excreted into the urine. After high-dose cytarabine, only 4-6\% of the dose was excreted unchanged in the urine. $63-73 \%$ appeared as ara- $U$ within the first $24 \mathrm{~h}$ [47]. 


\section{Intracellular metabolism and mechanism of action}

Like the other pyrimidine analogues, cytarabine has to enter the tumour cell, where it has to be activated by phosphorylation (Fig. 2). Cellular uptake of physiological nucleosides and nucleoside analogues is mediated by a family of integral membrane proteins termed human nucleoside transporters (hNTs). Two types of hNTs are distinguished: equilibrative and concentrative nucleoside transporters (hENTs and hCNTs, respectively). hENTs mediate the equilibrative movements of nucleosides in two directions. hCNTs co-transport nucleosides and sodium down their electrochemical gradients to concentrate nucleosides in the cells. Four hENTs (hENT1, hENT2, hENT3, and hENT4) and three hCNTs (hCNT1, hCNT2, and hCNT3) have been identified [51-53]. Cytarabine is transported into the cell primarily by hENT 1 [54].

Once in the cell, cytarabine is phosphorylated to its monophosphate (ara-CMP) by deoxycytidine kinase ( $d C K$ ) and then to cytarabine diphosphate (ara-CDP) by pyrimidine nucleoside monophosphate kinase (UMP-CMP kinase). The final phosphorylation step to cytarabine triphosphate (ara-CTP) is catalysed by nucleoside diphosphate kinase.

Ara-CTP competes with deoxycytidine triphosphate (dCTP) for incorporation into the DNA. Once incorporated into DNA, further extension of the DNA strand by DNA polymerase is inhibited. Correlations have been found between cytarabine incorporation into DNA and cytotoxicity [55-57].

Also within the cell, cytarabine is subject to deamination by cytidine deaminase. Besides, ara-CMP can be deaminated by deoxycytidylate deaminase [58].

\section{Clinical studies investigating the intracellular cytarabine nucleotide concentrations}

\section{Relation between cytarabine dose, plasma concentrations and intracellular ara-CTP concentrations}

Several clinical studies investigated the relationship between the cytarabine dose, the plasma PK and the intracellular PK of ara-CTP in leukemic cells [59-62]. A linear relationship was found between the cytarabine dose and 
the steady-state concentrations and AUCs of cytarabine and ara- $U$ in plasma. However, the intracellular pharmacokinetics of ara-CTP were much more heterogeneous [60-62]. No correlation was found between cytarabine plasma concentrations and the intracellular concentrations or AUC of ara-CTP [59]. Patients who had all received an identical cytarabine dose demonstrated substantial differences in the intracellular concentrations and AUCs of araCTP $[60,61]$. However within the same patient, measurements of the ara-CTP concentrations were reproducible with subsequent treatments [63]. The ability of leukemic cells to phosphorylate cytarabine is characteristic to each individual [59]. Inter-individual differences in the ability of patients to phosphorylate cytarabine and retain ara-CTP are related to the ability to transport cytarabine into the cell and the activities of deoxycytidine kinase, cytidine deaminase and dephosphorylating enzymes.

When examining the PK of intracellular ara-CTP there are also commonalities. The accumulation of ara-CTP in leukemic cells was dependent on the dose regimen, however, for an individual patient, the cellular elimination rate of araCTP remained constant under all infusion conditions. The elimination of ara-CTP from leukemic cells was monophasic. The elimination rate differed substantially between patients. In the study of Liliemark et al. a median $\mathrm{t}_{1 / 2}$ of $3.4 \mathrm{~h}$ (range, 1.25 to $18.9 \mathrm{~h}$ ) was found [59].

\section{Saturation of the intracellular accumulation of ara-CTP}

The accumulation of ara-CTP in leukemic cells was dependent on the dose regimen. In vitro studies in human leukaemia cells demonstrated that the intracellular accumulation of ara-CTP was saturated at cytarabine concentrations above $10 \mu \mathrm{mol} / \mathrm{L}[58,64,65]$. Plunkett et al. (1987) studied the saturation of intracellular ara-CTP formation in patients and investigated which cytarabine infusion-rate produced steady-state plasma concentrations that saturate the intracellular ara-CTP accumulation [60]. To this end, the intracellular ara-CTP accumulation in leukemic cells of 11 patients with refractory leukaemia was studied after different cytarabine doses infused over 2 hours. Varying the cytarabine dose between 500 and $3000 \mathrm{mg} / \mathrm{m}^{2}$ infused over 2 hours did not substantially change the accumulation rate and AUC of ara-CTP in leukemic cells. Only when the infusion rate was further reduced to 400 or $300 \mathrm{mg} / \mathrm{m}^{2}$ 
over 2 hours, resulting in steady-state cytarabine plasma concentrations of less than $7 \mu \mathrm{M}$, the accumulation rate of ara-CTP was substantially reduced as was the AUC of intracellular ara-CTP [60]. This study demonstrated that infusion rates of 200 to $250 \mathrm{mg} / \mathrm{m}^{2} / \mathrm{h}$ produce adequate levels of cytarabine in plasma to saturate the intracellular ara-CTP accumulation.

\section{Optimization of dosing regimens in relation to the intracellular accumulation of ara-CTP}

It is interesting to evaluate how the dosing regimens of cytarabine have evolved over time and how this relates to what we know about the intracellular accumulation of ara-CTP. As an example, we will focus on the cytarabine dosing regimens used in the treatment of $A M L$. Originally, cytarabine was used in remission induction therapy at a dose of $100-200 \mathrm{mg} / \mathrm{m}^{2}$ for $7-10$ days [66]. In the later literature this is referred to as conventional-dose cytarabine therapy.

In the 1980s, investigators began evaluating the use of high-dose cytarabine therapy with a dose of $3000 \mathrm{mg} / \mathrm{m}^{2}$ twice daily infused over 1 to 3 hours [6771]. These high-dose regimens resulted in peak plasma concentrations which were approximately 5 - to 15 -fold higher than the plasma concentrations that were found to saturate the accumulation of ara-CTP in leukemic cells [59,6769]. Substantial toxicities were associated with these high-dose cytarabine regimens [72-76]. It is likely that plasma cytarabine in excess of the amount that can be phosphorylated by leukemic cells, will contribute to the observed toxicity, either directly or after deamination to ara- $U[61,77]$.

After the study of Plunkett et al. in 1987, it would have been appropriate, from a pharmacological point of view, to evaluate intermediate infusion rates of 200 to $250 \mathrm{mg} / \mathrm{m}^{2} / \mathrm{h}$ as an alternative to the substantially higher cytarabine infusion rates that were used until then. The idea is that these intermediate infusion rates would still have saturated the intracellular ara-CTP formation in leukemic cells, but would have resulted in a substantial decrease of the peak plasma levels of cytarabine and ara- $U$ and, therefore, possibly might have led to less toxicity [78]. Nevertheless, the leading studies of that time continued to give high-dose cytarabine at a high infusion rate. Typically used high-dose regimens delivered $3 \mathrm{~g} / \mathrm{m}^{2}$ over 1 to $3 \mathrm{~h}$ at infusion rates of $1000-3000 \mathrm{mg} / \mathrm{m}^{2} / \mathrm{h}$. 
In the years after, these high-dose cytarabine regimens proved to be more effective than conventional-dose cytarabine [79-81]. High-dose cytarabine therapy administered at a high infusion rate became common practice in the treatment of AML, especially in patients younger than 60 years, either for remission induction or consolidation [82-84].

More than two decades later, investigators wondered whether such a large dose increase (from a conventional-dose of 200 to a high-dose of $3000 \mathrm{mg} / \mathrm{m}^{2}$ ) was really necessary. Due to the severe side effects of high-dose cytarabine observed in some patients with AML, clinical investigators initiated studies with an intermediate dose of cytarabine in the range of 1000 to $2000 \mathrm{mg} / \mathrm{m}^{2}$ [85]. In 2011, the HOVON-SAKK cooperative group performed a study in which two remission induction regimens, a high-dose and an intermediate-dose regimen, were compared in 860 patients with newly diagnosed AML between 18 and 60 years of age [86]. Patients were treated with two consecutive induction cycles. The high-dose group received cytarabine $1000 \mathrm{mg} / \mathrm{m}^{2}$ as a $3 \mathrm{~h}$-infusion twice daily for 5 days (cycle 1 ) and $2000 \mathrm{mg} / \mathrm{m}^{2}$ as a $6 \mathrm{~h}$-infusion twice daily for 4 days (cycle 2). The intermediate-dose group received cytarabine $200 \mathrm{mg} / \mathrm{m}^{2}$ by continuous infusion for 7 days (cycle 1 ) and $1000 \mathrm{mg} / \mathrm{m}^{2}$ as a $3 \mathrm{~h}$-infusion twice daily for 6 days (cycle 2). Patients with a complete response did not receive additional cytarabine but received consolidation therapy in a third cycle of chemotherapy (mitoxantrone-etoposide) or underwent autologous or allogeneic stem-cell transplantation. Evaluating the effect, no significant differences were found between the high- and the intermediate-dose group. However, the high-dose regimen did result in higher incidences of grade 3/4 toxicity, delayed neutrophil and platelet recovery and prolonged hospitalization [86]. Remarkably, the infusion durations in this study were chosen so that the maximum infusion rate, for both the high-dose and the intermediate-dose group, was $333 \mathrm{mg} / \mathrm{m}^{2} / \mathrm{h}$. This is just above the infusion rate of $250 \mathrm{mg} / \mathrm{m}^{2} / \mathrm{h}$, which would presumably saturate the intracellular ara-CTP formation [60]. This means for both dosing regimens that high plasma peaks were avoided and that both dosages could be effectively phosphorylated. Nevertheless, the intermediate dose already produced maximal anti-leukemic effects. This suggests a plateau in the dose-response relationship above a dose level of 1000 $\mathrm{mg} / \mathrm{m}^{2}$. Higher cytarabine doses resulted in excessive toxic effects without therapeutic benefit. After this study, intermediate-dose cytarabine regimens 
became common practice in the remission induction therapy of AML patients younger than 60 years.

Looking back on the studies aimed at optimizing the dosing regimen of cytarabine in AML, it is clear that a lot of attention has been paid to the administered dose, which was categorized as low, intermediate or high. However, much less attention has been paid to the administration rates. In a number of studies the infusion rates were not even mentioned. This is particularly striking in view of the pharmacology of cytarabine and the preclinical and clinical studies, performed in the 1980s, that demonstrated saturation of the intracellular ara-CTP formation. Not only the dose determines how much cytarabine is converted into active metabolites in the leukemic and healthy cells. The rate of administration is also decisive, particularly in view of the relatively short plasma half-life of cytarabine. This seems to have been forgotten for a considerable time.

\section{Relationship between intracellular ara-CTP concentrations and efficacy}

Several studies investigated the relationship between the accumulation of araCTP in leukemic cells and the response to cytarabine therapy.

Kantarjian et al. (1986) and Estey et al. (1987) investigated the relationship between the intracellular PK of ara-CTP in circulating blasts and the response of patients with refractory $A M L$ to remission induction therapy $[63,87]$. Patients were treated with 'high-dose cytarabine' and received $3000 \mathrm{mg} / \mathrm{m}^{2}$ infused over 2 hours every 12 hours for 4-12 doses. Intracellular ara-CTP levels were measured after the first dose and differed largely among patients. In the first study a trend was observed and in the follow-up study a significant correlation was found between the AUC of intracellular ara-CTP and the complete remission rate. Besides the AUC, also a long half-life of ara-CTP and a high araCTP trough concentration correlated with a high complete remission rate. The number of patients achieving a complete remission was too small to investigate the association between the PK of ara-CTP and the remission duration $[63,87]$.

By contrast, in the subsequent study of Estey et al. (1990) no correlation was found between the AUC of intracellular ara-CTP and the remission rate or remission duration [88]. This discrepancy is probably related to the fact that 
the PK of ara-CTP was investigated for all patients after a single gift of highdose cytarabine (3000 mg/m² infused over 2 hours), whereas patients were subsequently treated according to three different treatment protocols (i.e. continuous infusion of a conventional dose, bolus infusion of a high dose or continuous infusion of a high dose of cytarabine), which would probably result in different intracellular ara-CTP levels. For instance, for the subgroup of patients treated with high-dose cytarabine by continuous infusion, the steady state concentrations of ara-CTP did correlate with the remission rate and remission duration [88].

Rustum (1979) and Preisler (1985 and 1987) did not measure the intracellular ara-CTP concentrations during cytarabine therapy, but isolated leukemic blasts from more than 80 patients with AML prior to therapy, incubated these blasts with $\left[{ }^{3} \mathrm{H}\right]$-cytarabine and determined the percentage of ara-CTP that was retained after 4 hours [89-91]. Then, they studied the relationship between the in vitro retention of ara-CTP in the isolated blasts and patients' response to subsequent therapy with cytarabine and an anthracycline. Since the araCTP formation in leukemic blasts was studied after incubation and not during treatment with cytarabine, this series of three studies did not meet the inclusion criteria of this review. Therefore the results were not included in Tables 1 and 2. Nevertheless, we would like to discuss the results of this series here, since they have contributed to a better understanding of the relationship between intracellular ara-CTP retention and the efficacy of cytarabine therapy. The design of the studies was not appropriate to examine the relationship between the initial ara-CTP formation and the probability of achieving a complete remission, since patients received continuous cytarabine infusions during induction therapy. However, all three studies did find a significant correlation between the in vitro retention of ara-CTP in blasts and the duration of remission of patients receiving cytarabine as part of their remission induction and maintenance therapy [89-91].

The third study in this series compared the efficacy of three treatment regimens that were used for the treatment of AML between 1975 and 1983 [91]. In this study, the remission induction phase was similar for all patients, but after complete remission was achieved three follow-up regimens were compared. Regimen A consisted of two courses of consolidation therapy followed by five 
years of maintenance therapy, in which conventional subcutaneous cytarabine doses of $100 \mathrm{mg} / \mathrm{m}^{2}$ BID were administered for 5 consecutive days. Regimen $B$ was a bit more intensive and consisted of three consolidation cycles followed by three years of maintenance therapy, in which cytarabine was administered for 7 consecutive days. Regimen C was the most intensive as continuous cytarabine infusions were applied as well as high cytarabine doses of 3000 $\mathrm{mg} / \mathrm{m}^{2}$ BID during four cycles of consolidation therapy, followed by no further therapy. Prior to treatment, bone marrow cells were collected from all patients and incubated with cytarabine to study the intracellular ara-CTP formation and retention. Based on this incubation experiment, patients were divided into a low $(<20 \%)$ and high ( $\geq 20 \%)$ ara-CTP retention group. When treated with follow-up regimen $A$ or $B$, patients whose leukemic cells exhibited high retention of ara-CTP had longer remissions than individuals in the low-retention group. In contrast, ara-CTP retention was not of prognostic significance for patients treated with the more intensive regimen $\mathrm{C}$. This study demonstrated that intensification of the dose regimen improved the median remission duration. Median remission durations were 12, 17, and 22 months, respectively for the three treatment groups. The major benefit derived from increasing the intensity of therapy was experienced by patients with low ara-CTP retention. Whereas, the remission durations for patients in the high ara-CTP retention category were quite similar regardless of the treatment protocol. This study demonstrated that the adverse prognostic significance of low leukemic cell ara-CTP retention can be overcome by intensive consolidation therapy, especially therapy in which cytarabine is given in high doses [91].

\section{The role of arabinosyluracil triphosphate (ara-UTP) during cytarabine therapy}

Gandhi et al. (1998) demonstrated that, during cytarabine (1- $\beta$-Darabinosylcytosine; ara-C) therapy, also arabinosyluracil 5'-triphosphate (araUTP) was formed in circulating leukemic blasts [50]. The intracellular PK of araUTP was investigated in six patients with relapsed $A M L$ receiving cytarabine infusions of $500 \mathrm{mg} / \mathrm{m}^{2} / \mathrm{h}$ during 2 to $4 \mathrm{~h}$. The intracellular peak concentrations of ara-UTP ranged from 6 to $50 \mu \mathrm{M}$ and were between 2 and $10 \%$ of the peak concentrations that were measured for ara-CTP. Ara-UTP was maintained for several hours after the end of the cytarabine infusion. The intracellular ara- 
UTP peak levels were not related to the peak levels of cytarabine and ara- $U$ in plasma. However, a linear relationship was found between the intracellular ara-CTP and ara-UTP peak levels. These data indicate that the formation of ara-UTP may be dependent on ara-CTP and not on the free nucleoside [50].

This is in line with previous in vitro studies. Incubation of leukemic blasts with up to $100 \mu \mathrm{M}\left[{ }^{3} \mathrm{H}\right]$-ara-U did not result in ara-UTP accumulation, indicating that ara- $U$ is not phosphorylated directly in these cells [92]. In vitro studies, investigating the route of ara-UTP formation, showed that ara-UTP was formed through the deamination of ara-CMP to ara-UMP by deoxycytidylate deaminase, followed by phosphorylation to ara-UTP [50].

Question is whether ara-UTP also contributes to the cytotoxic effects of cytarabine. In vitro studies in leukemic cells demonstrated that ara-UTP served as a good alternative substrate for dTTP for incorporation into DNA [93] and inhibited DNA polymerase $[94,95]$. Hence, it could be postulated that ara-UTP may play a role in cytarabine-induced cytotoxicity $[94,96]$.

\section{Cytarabine in conclusion}

Although a linear relationship exists between the cytarabine dose and the plasma exposure, no correlation was found between the plasma exposure and the intracellular exposure of leukemic cells to ara-CTP [59-62]. Patients who all received an identical cytarabine dose demonstrated substantial differences in the intracellular concentrations and AUCs of ara-CTP $[60,61]$. What could be generally stated was that ara-CTP remained in the cell for a longer time than that cytarabine was present in the plasma. The median intracellular half-life $\left(t_{1 / 2}\right)$ of ara-CTP was $3.4 \mathrm{~h}$ (range, 1.25 to $18.9 \mathrm{~h}$ ), whereas the initial plasma $t_{1 / 2}$ of cytarabine was just 7 to 20 minutes $[47,48,59]$.

The accumulation of ara-CTP in leukemic cells depends also on the cytarabine dose regimen. Plunkett et al. demonstrated in 1987 that infusion rates of 200 to $250 \mathrm{mg} / \mathrm{m}^{2} / \mathrm{h}$ produced steady state plasma concentrations, just above $7 \mu \mathrm{M}$, that saturated the intracellular accumulation of ara-CTP in leukemic cells [60]. 
Despite this finding, high-dose cytarabine regimens, giving $3000 \mathrm{mg} / \mathrm{m}^{2}$ twice daily over 1 to $3 \mathrm{~h}$, were common practice in the treatment of AML for a long time. These regimens resulted in peak plasma concentrations that were approximately 5 - to 15 -fold higher than the plasma concentrations that were found to saturate the intracellular ara-CTP formation [59,67-69]. Substantial toxicities were associated with these high-dose regimens [72-76]. It was not until 2011, that intermediate-dose remission induction regimens were compared with these high-dose regimens and proved to be equally effective, but were associated with less toxicities [86]. Although many studies have been conducted over the last 30 years to find the most suitable cytarabine doses, little attention has been paid to the infusion rates. Yet, these infusion rates also determine how much cytarabine is converted intracellularly into ara-CTP.

Several studies demonstrated a clear relationship between the accumulation of ara-CTP in leukemic cells and the response to cytarabine therapy [87]. The AUC of ara-CTP measured in leukemic blasts during high-dose cytarabine therapy was correlated with the probability of achieving a complete remission in patients with refractory acute leukaemia [87]. In addition, the in vitro retention of araCTP in isolated leukemic blasts was correlated with the duration of remission in patients subsequently treated with cytarabine and an anthracycline [89-91]. However, the adverse prognostic significance of low leukemic cell ara-CTP retention could be overcome by intensive consolidation therapy, which included high cytarabine doses or the administration of conventional-dose cytarabine by continuous infusion [91]. 


\section{GEMCITABINE}

Gemcitabine has been used as a chemotherapeutic agent since 1995. Although structurally similar to cytarabine, gemcitabine has a different spectrum of anti-tumour activity, and shows cytotoxicity in a variety of solid tumours. Nowadays gemcitabine has an important place in the treatment of advanced pancreatic, bladder, breast, ovarian and non-small cell lung cancer. Gemcitabine (2',2'-difluoro-2'-deoxycytidine, dFdC) is a deoxycytidine analogue in which the two hydrogen atoms at the 2'-position of the deoxyribose moiety are replaced by fluorine atoms.

\section{Plasma PK of gemcitabine}

During standard gemcitabine infusions, administered over 30 minutes, gemcitabine plasma concentrations generally reached a plateau level within 15 minutes from start of the infusion [97]. The plasma concentration and the AUC of gemcitabine in plasma were proportional to the dose that was administered (over a dose range of 53 to $1000 \mathrm{mg} / \mathrm{m}^{2}$ ) [97]. Gemcitabine is rapidly cleared from plasma with a median $t_{1 / 2}$ of $8 \mathrm{~min}$. Metabolic clearance by deamination was identified as the major form of elimination. More than $90 \%$ of the gemcitabine dose is converted into 2',2'-difluoro-2'-deoxyuridine (dFdU) by cytidine deaminase [97]. The activity of cytidine deaminase is the most important factor determining the elimination rate of gemcitabine [46]. The $\mathrm{C}_{\max }$ of $\mathrm{dFdU}$ was generally observed 5 to 15 minutes after the end of the gemcitabine infusion and was also proportional to the dose. $\mathrm{dFdU}$ is excreted into the urine. Plasma kinetics of dFdU exhibited a biphasic elimination, characterized by a median initial $t_{1 / 2}$ of 27 min and a terminal elimination phase that was generally quite long (median $t_{1 / 2 \beta}, 14 \mathrm{~h}$ ), with considerable variation among patients (range, 2.5 to $>24$ h) [97].

\section{Intracellular metabolism and mechanism of action}

Like the other pyrimidine analogues, gemcitabine has to enter the tumour cell, where it has to be activated by phosphorylation (Fig. 2). Cellular uptake of gemcitabine is largely mediated by hENT 1 and, to a lesser extent, also by hENT2 and hCNT 1 and 3 [51,52]. Once inside the cell, gemcitabine (dFdC) 
is phosphorylated in the cytoplasm by dCK to its monophosphate (dFdCMP) and then by pyrimidine nucleoside monophosphate kinase (UMP-CMP kinase) to gemcitabine diphosphate (dFdCDP). The enzyme responsible for the final phosphorylation step, the phosphorylation of dFdCDP to gemcitabine triphosphate (dFdCTP) is unclear, although nucleoside diphosphate kinase may play this role [98].

Intracellularly formed dFdCTP and dFdCDP are responsible for the cytotoxic effects of gemcitabine. dFdCTP competes with dCTP for incorporation into the DNA. Incorporation of dFdCTP into DNA leads to inhibition of the DNA synthesis and apoptosis of the cell $[99,100]$. The mechanism of action of gemcitabine is self-potentiated by inhibition of two enzymes involved in the deoxynucleotide metabolism. dFdCDP inhibits the enzyme ribonucleotide reductase which catalyses the reduction of ribonucleotides to deoxyribonucleotides [101]. By inhibiting this enzyme, the intracellular deoxyribonucleotide triphosphate (dNTP) pool is depleted. This increases the dFdCTP : dCTP ratio, which favours the incorporation of dFdCTP into the DNA. This effect is enhanced in several ways. dFdCTP directly inhibits the enzyme deoxycytidylate deaminase [102]. The same enzyme is also inhibited indirectly by the reduced intracellular dNTP pool. Since dCK activity is also regulated by dCTP, lowering the dNTP pool also promotes gemcitabine phosphorylation $[103,104]$.

Like in plasma, also in the cell, a major form of elimination of gemcitabine is deamination to dFdU by cytidine deaminase. Likewise, dFdCMP is deaminated by deoxycytidylate deaminase $[102,105]$. Gemcitabine can also become inactivated by dephosphorylation of the monophosphate form by 5'-nucleotidases (5'-NTs), converting nucleotides back to nucleosides [103].

\section{Intracellular PK of gemcitabine - Important lessons from preclinical studies}

In vitro studies demonstrated that the cellular uptake of gemcitabine by hENT1, hENT2 and hCNT1 was saturable and conformed to Michaelis-Menten kinetics $[51,52,106]$. Apparent Michaelis-Menten constant $\left(K_{m}\right)$ values of 160,740 and $24 \mu \mathrm{M}$ were found for hENT1, hENT2 and hCNT1, respectively [106]. 
In vitro studies with radioactive gemcitabine demonstrated that the triphosphate was the major intracellular metabolite. Relatively small concentrations of either the monophosphate or the diphosphate were present in human cells [102]. This indicated that phosphorylation of gemcitabine to dFdCMP by dCK was the rate-limiting step in the formation of dFdCTP $[107,108]$ Gemcitabine was found to be a good substrate for phosphorylation by dCK, having a $K_{m}$ value of 5-10 $\mu \mathrm{M}[107,109]$. The rate of phosphorylation became saturated at gemcitabine concentrations above $20 \mu \mathrm{M}[107,108]$.

In vitro and in vivo studies with murine tumours demonstrated a biphasic elimination of dFdCTP, with a terminal $t_{1 / 2}$ of $12 \mathrm{~h} \mathrm{[102].} \mathrm{The} \mathrm{elimination} \mathrm{was}$ found to be dependent of the intracellular concentration of dFdCTP. Cells incubated with dFdCTP concentrations up to $100 \mu \mathrm{M}$ demonstrated monophasic elimination, whereas cells that contained more than $100 \mu \mathrm{M} \mathrm{dFdCTP}$ tended to exhibit biphasic elimination patterns with more prolonged terminal elimination phases. Deamination was found to be the predominant means of catabolism in cells containing less than $100 \mu \mathrm{M}$ dFdCTP, whereas dephosphorylation and excretion of gemcitabine was the major elimination route in cells containing higher triphosphate levels [102].

\section{Clinical studies investigating the intracellular gemcitabine nucleotide concentrations}

\section{Saturation of the intracellular accumulation of dFdCTP}

The intracellular metabolism of gemcitabine follows the same pathways as that of cytarabine (Fig. 2). Therefore, in the early 90 s, the intracellular accumulation of dFdCTP was expected to exhibit similar saturation kinetics as previously demonstrated for ara-CTP [110]. Grunewald et al. confirmed that the ability of PBMCs to convert gemcitabine into dFdCTP was saturable. In a phase I study, including 22 patients with solid tumours, gemcitabine doses between 53 and $1000 \mathrm{mg} / \mathrm{m}^{2}$ (all infused over $30 \mathrm{~min}$ ) were compared. The rate of dFdCTP accumulation in PBMCs initially increased with higher doses, but was found to be maximal at a dose of $350 \mathrm{mg} / \mathrm{m}^{2}$ infused over $30 \mathrm{~min}$. At this dose rate, gemcitabine steady state plasma concentrations of 15-20 $\mu \mathrm{M}$ were measured. Although the study demonstrated considerable inter-individual variation, above 
a gemcitabine steady state concentration of $20 \mu \mathrm{M}$, no further increase of the dFdCTP AUC was found for the majority of patients [97,110].

A subsequent phase I study, involving 17 patients with leukaemia, attempted to maximize the accumulation of dFdCTP in leukemic cells [111]. To this end, gemcitabine was administered at an infusion rate that would presumably saturate the intracellular dFdCTP formation. An infusion rate of $10 \mathrm{mg} / \mathrm{m}^{2} / \mathrm{min}$ was selected. This infusion rate was previously found to produce steady state plasma concentrations above 15-20 $\mu \mathrm{M}$ [112]. In this study the dose intensity was increased by prolonging the duration of the infusion at this fixed dose rate (from 120 to 640 minutes in six steps). The maximum-tolerated dose found in this study was $4800 \mathrm{mg} / \mathrm{m}^{2}$ infused over 480 minutes weekly for three weeks. The mean steady state plasma concentration of gemcitabine during infusion at $10 \mathrm{mg} / \mathrm{m}^{2} / \mathrm{min}$ was $26.5 \mu \mathrm{M}$ with a range of 13.0 to $53.5 \mu \mathrm{M}$. Only four of the 17 patients had steady state plasma levels below $20 \mu \mathrm{M}$. For 12 of the 15 assessable patients, linear accumulation of intracellular dFdCTP was seen during the infusion of gemcitabine. In the remaining three patients, the intracellular dFdCTP accumulation reached a plateau during the infusion. The accumulation rate of dFdCTP in circulating leukemic cells varied considerably among individuals, ranging from 9.7 to $789 \mu \mathrm{mol} / \mathrm{L} / \mathrm{h}$. Consistent with findings from preclinical studies, the elimination of $\mathrm{dFdCTP}$ was related to its intracellular concentration. Blasts with more than $450 \mu \mathrm{M}$ dFdCTP showed biphasic elimination, whereas blasts with lower dFdCTP concentrations demonstrated linear elimination [111].

\section{Increasing the intracellular accumulation of dFdCTP by prolonging the infusion time}

The aforementioned clinical trials suggested that the intracellular accumulation of dFdCTP could be increased by giving more prolonged gemcitabine infusions at an infusion rate that maintains gemcitabine plasma concentrations which saturate the formation of dFdCTP. Since then, abundant evidence has been published that the intracellular accumulation of dFdCTP in PBMCs and leukemic blasts indeed increased by prolonging the infusion time (Table 2) [113-115]. 
Patel et al. performed a cross-over study, in which seven evaluable patients received gemcitabine $1000 \mathrm{mg} / \mathrm{m}^{2}$ as a standard 30 -minute infusion in week 1 and as a 150-minutes infusion in week 2 [113]. After the 30-minute infusion, the dFdCTP concentration measured in PBMCs reached a plateau within 30-120 min. Whereas during the 150-minutes infusion, the dFdCTP accumulation remained linear throughout the entire infusion. Maximum dFdCTP concentrations were significantly higher following the 150-minutes infusion compared with the 30-minute infusion (median increase 1.4-fold, range 1.0 to 2.6-fold) [113].

Cattel et al. compared gemcitabine $300 \mathrm{mg} / \mathrm{m}^{2}$ infused over 1,2 or $3 \mathrm{~h}$ with a conventional gemcitabine dose of $1000 \mathrm{mg} / \mathrm{m}^{2}$ infused in $30 \mathrm{~min}$ [115]. Twenty patients affected by different solid tumours were randomized over the four treatment arms. Measurements of the intracellular dFdCTP concentrations in leukocytes showed that gemcitabine $300 \mathrm{mg} / \mathrm{m}^{2}$ infused over $3 \mathrm{~h}$ produced the highest accumulation of dFdCTP. Indicating that, to achieve the highest possible dFdCTP levels, a prolonged infusion time was more important than a high dose administered as a short infusion [115].

Grimison et al. performed a cross-over study in which 31 patients received a gemcitabine dose of $1000 \mathrm{mg} / \mathrm{m}^{2}$ over either 30 or $100 \mathrm{~min}$ [116]. At the second week, each patient received the alternate schedule. Intra-patient comparison showed that the intracellular dFdCTP exposure in leucocytes was significantly higher following the 100-minute infusion. However, the effect was confounded by an increase in dFdCTP exposure in the second week compared to the first week, which was consistent with self-induction of the gemcitabine metabolism. Because of this effect, prolonged infusion only resulted in a significant increase of the dFdCTP exposure when the 30-minute infusion was given in week 1. Reversing the order of administration abolished the increase. Moreover, significant variations in intracellular dFdCTP exposure were observed both between and within individuals. We have to realize that self-induction of the gemcitabine metabolism (among others due to dCK activation) implicates that the optimal dose rate for saturation of the intracellular accumulation changes over time. Due to self-induction and inter-individual differences, a dose rate of $10 \mathrm{mg} / \mathrm{m}^{2} / \mathrm{min}$ will not be optimal for all patients all of the time [116]. 


\section{Relationship between intracellular dFdCTP concentrations and efficacy}

Although the abovementioned studies have focused on maximization of the intracellular dFdCTP concentration, no clear relationship in patients has been demonstrated between this intracellular dFdCTP concentration and effect or toxicity. This relationship has only been demonstrated in tumour cell lines and xenograft studies [117]. Various clinical studies compared the efficacy of prolonged administration of gemcitabine at a fixed dose rate (FDR) (e.g. 10 $\mathrm{mg} / \mathrm{m}^{2} / \mathrm{min}$ ) with the conventional 30-minute infusion of gemcitabine (1000$1250 \mathrm{mg} / \mathrm{m}^{2}$ ) [118-124]. However, although these studies were all based on the principle that prolonged infusion would maintain gemcitabine plasma concentrations that result in saturation of the intracellular dFdCTP formation, only a few studies actually measured the intracellular concentrations of dFdCTP (Table 2) $[116,118,121,124]$.

The majority of the performed phase II studies that compared FDR infusion of gemcitabine with conventional 30-minute infusion found no clear difference in efficacy [120-122]. It is important to note that the sample size of some of these studies was also not sufficient to compare efficacy [121]. In addition, the comparison was hampered as in some studies not only the dose-rate was different between the treatment arms, but also the total gemcitabine dose that was administered $[118,121]$. Only one randomized phase II study showed a survival advantage with prolonged infusion [118]. In this study, standard gemcitabine infusion ( $2200 \mathrm{mg} / \mathrm{m}^{2}$ in $30 \mathrm{~min}$ ) was compared with FDR infusion (1500 mg/m² in $150 \mathrm{~min}$ ) in 92 patients with advanced pancreatic cancer. The dose levels for both regimens were based on the maximum tolerated doses established in previous studies [125-127]. Measurement of the dFdCTP concentrations in PBMCs confirmed that the rate of dFdCTP accumulation decreased markedly after the end of the 30-minute infusion. Maximum dFdCTP concentrations were reached one hour after the end of the infusion and the median $\mathrm{C}_{\max }$ was $188 \mu \mathrm{M}$. In contrast, during the 150-minute FDR infusion, the dFdCTP concentration increased linearly during the infusion up to a median concentration of $398 \mu \mathrm{M}$ at the end of the infusion. Unfortunately, intracellular dFdCTP concentrations were not monitored over a longer period of time, thus the AUC values of dFdCTP could not be compared. Median survival was longer for patients in the FDR arm than for those in the standard arm (8.0 vs. 5.0 months; $p=.013$ ), and also the 1 - and 2-year survival rates were significantly 
higher following FDR infusion. It should be noted here that the comparison might be confounded by the fact that twice as many patients in the FDR arm as in the standard arm received second-line chemotherapy, which might have influenced survival. On the other hand, it is possible that more patients receiving FDR gemcitabine were able to proceed to second-line treatment based on a better performance status [118].

However, the confirmatory phase III trial in 832 patients with advanced pancreatic cancer failed to show a significant advantage with a prolonged infusion schedule [124]. In this study, three treatment arms were compared, namely standard gemcitabine $1000 \mathrm{mg} / \mathrm{m}^{2}$ infused in $30 \mathrm{~min}$ (GEM), FDR gemcitabine $1500 \mathrm{mg} / \mathrm{m}^{2}$ infused in $150 \mathrm{~min}$ (GEM-FDR), and gemcitabine $1000 \mathrm{mg} / \mathrm{m}^{2}$ infused in $100 \mathrm{~min}$ in combination with oxaliplatin (GEMOX). It should be noted that patients received a 1.5-fold higher gemcitabine dose in the GEM-FDR arm. The AUC of intracellular dFdCTP measured in PBMCs was significantly increased for both GEM-FDR and GEMOX compared to standard gemcitabine infusion (GEM; Table 2). However, neither GEM-FDR nor GEMOX resulted in a significantly improved overall survival, progression free survival or response rate compared to standard gemcitabine infusion [124].

In the phase III study of Gridelli et al., 440 patients with advanced non-small cell lung cancer were treated with a combination of cisplatin and gemcitabine and the effect of FDR gemcitabine $\left(1200 \mathrm{mg} / \mathrm{m}^{2}\right)$ infusion in $120 \mathrm{~min}$ was compared with standard 30-minute infusion. Also in this patient population, FDR infusion of gemcitabine did not improve overall survival, progression-free survival or the response rate compared with standard infusion. Unfortunately, this study did not examine the pharmacokinetics of gemcitabine or dFdCTP [123].

So far, there is thus no clear evidence that prolonging the infusion time of gemcitabine results in better treatment efficacy.

\section{Relationship between intracellular dFdCTP concentrations and toxicity}

Grimison et al. found a relationship between the AUC of dFdCTP in leukocytes and the degree of myelosuppression. When the dFdCTP samples were divided at the median $(81.0 \mu \mathrm{mol} /$ million cells $\times \mathrm{h}$ ) into a high and a low 
group, the high dFdCTP group was associated with a significant greater degree of myelosuppression. No correlation was found between the degree of myelosuppression and the $\mathrm{C}_{\max }$ or AUC of gemcitabine in plasma. The degree of myelosuppression after the first treatment week was greater for the group that received gemcitabine $1000 \mathrm{mg} / \mathrm{m}^{2}$ infused over 100-min than for the group that received 30-minute infusions, however this difference did not reach statistical significance [116]. In the study of Soo et al., the AUC of intracellular dFdCTP was similar after FDR infusion of gemcitabine and standard 30-minute infusion of a $25 \%$ higher gemcitabine dose. In this study, no correlation was found between the $\mathrm{C}_{\max }$ or AUC of intracellular dFdCTP and the percentage change in neutrophils or platelets [121].

Studies that did not examine the relationship between the intracellular dFdCTP accumulation and toxicity, but just compared the toxicity after FDR infusion versus conventional gemcitabine infusion, were not consistent. Some studies showed increased haematological toxicity after FDR infusion. In the study of Tempero et al., patients who received an FDR infusion (1500 mg/m² in $150 \mathrm{~min}$ ) experienced more haematological toxicities than patients treated with standard gemcitabine $\left(2200 \mathrm{mg} / \mathrm{m}^{2}\right.$ in $30 \mathrm{~min}$ ) [118]. Also in the study of Cappuzzo et al., FDR dosing $\left(1500 \mathrm{mg} / \mathrm{m}^{2}\right.$ in $\left.150 \mathrm{~min}\right)$ resulted in a higher incidence of grade 3 or 4 neutropenia than standard infusion of the same gemcitabine dose in 30 min (49.2\% vs. 17.9\%; $p<.001)$ [122]. In the phase III study of Poplin et al., FDR-infusion of gemcitabine also caused more grade 3/4 neutropenia and thrombocytopenia than standard 30-minute infusion, but the total gemcitabine dose that was administered during the FDR-infusion was 1.5 times higher [124]. However, there were also studies that showed no significant difference in toxicity $[119,123]$. In the phase III study of Gridelli et al., no significant difference was noted in the occurrence and severity of most toxicities. Only vomiting and hair loss were significantly more frequent in the FDR-arm [123]. In the other studies, non-haematological toxicities were similar for both infusion strategies and consisted mainly of nausea and vomiting [118,119,121,122,124].

As mentioned, it is difficult to assess the separate effect of the infusion rate, since in many studies, also the total dose that was administered is different for the compared treatment arms [118,121,124]. It is evident that there is a strong link between the maximum tolerated dose (MTD) and the infusion 
schedule. Considering the phase I dose-escalation studies performed in a chemotherapy-naive patient population, it can be said that FDR infusion of gemcitabine resulted in a lower MTD than conventional 30-minute infusion (1500-1800 mg/m²/week vs. $2200 \mathrm{mg} / \mathrm{m}^{2} /$ week; for 3 weeks every 4 weeks) due to myelotoxicity [125-127].

\section{Interpretation and future perspectives}

Prolonged gemcitabine infusions at a FDR aim at a more efficient accumulation of the intracellular anabolites dFdCDP and dFdCTP. If we assume that both the antineoplastic effects and the toxic effects of gemcitabine are equally and exclusively mediated by dFdCDP and dFdCTP, then prolonged infusion would result in a lower maximum tolerated dose (MTD). When, in the outlined situation, FDR infusion is adjusted to this lower MTD, no increase in efficacy is to be expected.

Based on this rationale, prolonged FDR infusions would only be useful if the mechanisms underlying toxicity do not completely parallel the mechanisms that are responsible for the antineoplastic effects of gemcitabine. One could think of differences in accumulation rate between target tissues and healthy tissues due to different membrane transporter capacities or enzyme capacities, differences in cell division rate, but also of other gemcitabine metabolites that might contribute to the dose-limiting toxicities. In this light, the role of dFdU could be interesting.

\section{The role of $\mathrm{dFdU}$ nucleotides during gemcitabine therapy}

Although dFdU was traditionally believed to be an inactive metabolite, in vitro studies demonstrated that dFdU has cytotoxic activity in tumour cell lines [128130]. The $I C_{50}$ for $d F d U$ in gemcitabine sensitive tumour cell lines was at least a 1000 to 2000 fold higher than for gemcitabine [128-130]. However, during gemcitabine treatment, the exposure to dFdU highly exceeds the exposure to gemcitabine [97,131]. Therefore, the question is what is the contribution of dFdU to the cytotoxic effect of gemcitabine. 
In vitro studies in various cell lines demonstrated that dFdU is taken up into cells, for instance by hCNT1 [129]. In the cell, dFdU can be phosphorylated to its monophosphate (dFdUMP), diphosphate (dFdUDP) and triphosphate (dFdUTP) [129]. Alternatively, dFdCMP can be deaminated to dFdUMP, by deoxycytidylate deaminase, and then further phosphorylated [102,105]. In vitro studies also demonstrated that dFdUTP is incorporated into both DNA and RNA and that the extent of incorporation correlated with the cytotoxicity of dFdU [129].

In 2008 Veltkamp et al. performed a feasibility study with orally administered gemcitabine and demonstrated for the first time that, besides dFdCTP, also dFdUTP was formed in patient PBMC samples [132]. After oral administration, the systemic exposure to gemcitabine proved to be very low due to extensive first-pass metabolism to dFdU. Consistently, the intracellular dFdUTP exposure measured in PBMCs was significantly higher than the dFdCTP exposure. One patient died during the study experiencing grade 4 hepatic failure. It was noted that the exposure to dFdUTP was excessively high in this patient. This suggested that dFdUTP could have played a role in the toxicity experienced by this patient [132].

Because little was known about the intracellular formation of dFdUTP during intravenous gemcitabine treatment, we performed a study to explore the intracellular pharmacokinetics of both the $\mathrm{dFdC}$ nucleotides and the $\mathrm{dFdU}$ nucleotides (i.e. dFdUMP, dFdUDP and dFdUTP) during gemcitabine treatment [131]. Plasma and PBMC samples were collected from 38 patients receiving gemcitabine, at multiple time-points after infusion. Both in plasma and in PBMCs, dFdU was present in higher concentrations than gemcitabine. However, the dFdUMP, dFdUDP and dFdUTP concentrations in PBMCs were low in all patients; much lower than the dFdCDP and dFdCTP concentrations. The mean $A_{U-24 h}$ for dFdUTP was $312 \mu M^{*} h$ vs. $2640 \mu M^{*} h$ for dFdCTP. This finding suggests that $\mathrm{dFdU}$ might play a limited role in the cytotoxic effect of gemcitabine, because of limited intracellular activation to dFdU nucleotides [131]. 


\section{Combination regimens and intracellular PK interactions}

A number of studies that examined combination regimens with gemcitabine also assessed possible interaction effects on the intracellular PK of dFdCTP. Cisplatin was found to increase the intracellular accumulation of dFdCTP in PBMCs. Cisplatin induces DNA damage and thereby nucleotide excision repair-processes, which up-regulate several enzymes including deoxycytidine kinase [133]. Paclitaxel, which was administered 3 hours prior to gemcitabine, was also found to increase the intracellular accumulation of dFdCTP dosedependently compared to single-agent gemcitabine. No effects on the plasma pharmacokinetics of gemcitabine or dFdU were found [134]. The mechanism of this interaction has not been fully elucidated and is beyond the scope of this review.

\section{Gemcitabine in conclusion}

Its noteworthy that almost all intracellular PK studies were focused on dFdCTP. Although preclinical studies have shown that dFdCDP also contributes to the cytotoxic effect of gemcitabine, we have not found any clinical studies investigating the intracellular PK of dFdCDP.

Intracellular PK studies demonstrated that the accumulation of dFdCTP in PBMCs is saturable and reached a plateau when the gemcitabine plasma concentration exceeded 15 to $20 \mu \mathrm{M}$ [97,110]. Pilot studies with leukaemia patients suggested that a fixed-dose rate (FDR) of $10 \mathrm{mg} / \mathrm{m}^{2} / \mathrm{min}$ would produce plasma gemcitabine concentrations greater than $20 \mu \mathrm{M}$ and thus would maximize the rate of dFdCTP accumulation [112]. This plasma level and infusion rate have been shown to increase intracellular accumulation in PBMCs when compared with a standard 30-minute infusion $[113,115]$. However, to date there is no clear evidence that FDR administration leads to a significantly better treatment outcome compared with standard gemcitabine infusion [135]. Two issues might play a role here: I.) If mechanisms of action and toxicity are completely parallel, both equally and exclusively dependent of the intracellular accumulation of dFdCDP and dFdCTP, FDR infusion is associated with a lower maximum tolerated dose, abolishing the therapeutic benefits. II.) Large interindividual variations were demonstrated. One study that evaluated a $10 \mathrm{mg} /$ $\mathrm{m}^{2} / \mathrm{min}$ schedule found that 8 of 13 patients (62\%) achieved target plasma 
concentrations [136], which suggests that individualization of the dose rate may be required to achieve optimal dosing. Understanding and predicting the cause of this inter-patient variation could lead to individualized dosing and improved therapeutic efficacy. 


\section{AZACITIDINE AND DECITABINE}

In the last chapter of this review we will focus on the demethylating agents azacitidine (aza-C) and decitabine (5-aza-2'-deoxycytidine; aza-dC). Azacitidine and decitabine are analogues of cytidine and deoxycytidine respectively, in which the carbon atom at position 5 of the pyrimidine ring has been replaced by a nitrogen atom. The anti-leukemic effects of azacitidine and decitabine have been studied since the 1960s. However, it took until 2004 before azacitidine received the first market approval, followed by decitabine in 2006 . Both drugs were approved by the U.S. Food and Drug Administration (FDA) for the treatment of myelodysplastic syndrome (MDS). In 2008, azacitidine was also approved by the European Medicines Agency (EMA). In Europe, azacitidine is approved for the treatment of adult patients with higher-risk MDS, certain forms of chronic myelomonocytic leukaemia (CMML) and $A M L$, who are not eligible for hematopoietic stem cell transplantation. For decitabine, it took until 2012 before it received EMA approval. Interestingly, in Europe, decitabine was not approved for the treatment of MDS, but for the treatment of adult patients with newly diagnosed AML who are not candidates for standard induction chemotherapy.

\section{Plasma PK of azacitidine and decitabine}

Azacitidine is given as a subcutaneous injection into the upper arm, thigh or abdomen. The recommended starting dose is $75 \mathrm{mg} / \mathrm{m}^{2}$ daily for 7 consecutive days, with a cycle duration of 28 days. After subcutaneous administration, azacitidine is rapidly absorbed. Peak plasma concentrations were reached after 15-30 min $[137,138]$. The bioavailability of subcutaneous azacitidine relative to IV azacitidine was approximately 89\% [137]. The mean volume of distribution following subcutaneous administration was 259 L [138]. High interpatient variability was observed for the azacitidine plasma concentrations $[137,138]$. Azacitidine is cleared rapidly from plasma. It undergoes spontaneous hydrolysis and deamination to 5-aza-uridine (aza-U) mediated by cytidine deaminase $[139,140]$. Following subcutaneous administration of azacitidine, the mean $t_{1 / 2}$ values found in the two main studies were $41 \mathrm{~min}$ and $72 \mathrm{~min}$, respectively $[137,138]$. No plasma accumulation occurs after subcutaneous administration of $75 \mathrm{mg} / \mathrm{m}^{2}$ azacitidine once daily for 7 days [138]. Dose-proportionality was evaluated for subcutaneous azacitidine doses from 25 to $100 \mathrm{mg} / \mathrm{m}^{2} /$ day. Within 
this dose-range, the areas under the curve and maximum plasma concentrations were approximately proportional with the dose [138].

Other than azacitidine, decitabine is given by intravenous infusion. In Europe, the approved decitabine dosing schedule for the treatment of $A M L$ is $20 \mathrm{mg} / \mathrm{m}^{2}$ infused over $1 \mathrm{~h}$ daily for 5 consecutive days, with a cycle duration of 28 days. The approved decitabine dose in the U.S. for the treatment of MDS is $15 \mathrm{mg} / \mathrm{m}^{2}$ administered by intravenous infusion over $3 \mathrm{~h}$, repeated every $8 \mathrm{~h}$ for 3 days, with a cycle duration of 6 weeks. The plasma PK of decitabine was examined for the latter dosing schedule [141]. By the end of the infusion, steady state plasma concentrations appeared to have been reached. The mean volume of distribution at steady state was $75 \mathrm{~L} / \mathrm{m}^{2}$, indicating distribution into peripheral tissues [141]. Decitabine was rapidly eliminated from the plasma with a mean $t_{1 / 2}$ of 41 min [141]. The primary route of metabolism is through deamination to 5-aza-2'deoxyuridine (aza-dU) by cytidine deaminase $[139,142]$. Maximum plasma concentrations and AUCs remained unchanged for the repeated decitabine doses during the 3 days. Thus, the repeated dosing did not result in plasma accumulation of the drug. Also from cycle to cycle, the plasma PK remained unchanged [141].

\section{Intracellular metabolism and mechanism of action}

Cellular uptake of azacitidine is mediated by all seven human nucleoside transporters, with hCNT3 showing the highest transport rates [53]. Decitabine is transported into cells by hENT1 and 2 [53]. Since azacitidine is an analogue of cytidine and decitabine an analogue of deoxycytidine, different enzymes are involved in their intracellular metabolism (Fig. 2). Once in the cell, azacitidine is phosphorylated to 5-azacytidine monophosphate (aza-CMP) by uridinecytidine kinase and then to 5-azacytidine diphosphate (aza-CDP) and 5 -azacytidine triphosphate (aza-CTP) by UMP-CMP kinase and nucleoside diphosphate kinase, respectively. A small fraction, about 10\%-20\%, of the azaCDP is instead converted to 5-aza-2'-deoxycytidine diphosphate (aza-dCDP) via the enzyme ribonucleotide reductase, followed by further phosphorylation to 5-aza-2'-deoxycytidine triphosphate (aza-dCTP) [143-145]. Decitabine is, after uptake in the cell, converted to its monophosphate (aza-dCMP) by deoxycytidine kinase. dCMP kinase and nucleoside diphosphate kinase convert 
the monophosphate to its diphosphate form (aza-dCDP) and triphosphate form (aza-dCTP), respectively [142]. Like in plasma, azacitidine and decitabine are also in the cell primarily eliminated by deamination by cytidine deaminase. Likewise, aza-dCMP is deaminated to 5-aza-2'-deoxyuridine monophosphate (aza-dUMP) by deoxycytidylate deaminase [142].

Intracellularly formed aza-CTP and aza-dCTP are responsible for the pharmacological effects of azacitidine and decitabine. Aza-CTP is incorporated into RNA. This ultimately disrupts mRNA metabolism and protein synthesis, leading to apoptosis $[144,145]$. Besides incorporation of aza-CTP into RNA inhibits ribonucleotide reductase, leading to a reduced deoxyribonucleotide pool, which has deleterious consequences for DNA synthesis and repair [144]. Aza-dCTP competes with endogenous dCTP for DNA incorporation. At high doses, aza-dCTP incorporation into DNA leads to inhibition of DNA synthesis and cell death [142,146]. However, at lower doses, a smaller amount of azadCTP is incorporated into the DNA, which primarily causes inhibition of DNA methylation [142]. DNA methylation has an important role in the regulation of gene expression. Aberrant DNA methylation has a place in the pathogenesis of several malignancies including MDS and AML $[147,148]$. The most studied change of DNA methylation is the silencing of tumour suppressor genes by hypermethylation of the CpG islands within their promoter regions [149]. The enzymes responsible for DNA methylation are DNA methyltransferases (DNMTs). When aza-dCTP is incorporated into the DNA, covalent bonds are formed between the aza-dC in the DNA and these DNMTs. At high doses, the DNA is not able to recover and cell death occurs. However, at lower doses the formed DNA-DNMT adducts are degraded by the proteosome, after which the DNA is restored. DNA synthesis is then resumed in the absence of DNMTs. As a consequence the aberrant DNA hypermethylation patterns, which play an important role in the pathogenesis of the mentioned hematologic abnormalities, can no longer be reproduced toward the daughter strands [150]. In this way, a low dose of azacitidine or decitabine is able to induce re-expression of previously silenced genes. Reactivation of cell cycle-regulating genes (i.e. tumour suppressor genes), that were initially silenced due to hypermethylation, may restore cell differentiation and normal growth control in the daughter cells $[145,151,152]$. 


\section{Clinical studies investigating the intracellular azacitidine and decitabine nucleotide concentrations}

In the 1970s and 1980s, the anti-leukemic effects of azacitidine and decitabine were studied in high doses based on the maximum tolerated dose. The antineoplastic effect of these high doses was based on cytotoxicity. From the 1990s, researchers also started to study low azacitidine and decitabine doses based on the idea of their demethylating effect [153-156]. It was only at these low doses that the therapeutic benefits of azacitidine and decitabine were demonstrated $[157,158]$. Both drugs were therefore registered in low doses. Few clinical studies have examined the intracellular pharmacokinetics of the active metabolites aza-CTP and aza-dCTP. Other than for the other pyrimidine analogues described in this review, for low-dose azacitidine and decitabine therapy, we are not interested in maximizing intracellular triphosphate concentrations to maximize the effect. The optimal dose is more nuanced and comes down to the dose that maximizes the demethylating effect without the cytotoxic effect prevailing.

Only recently, three studies investigated the intracellular pharmacokinetics of the active metabolites aza-CTP and aza-dCTP in patients that were treated with azacitidine or decitabine [159-161]. The main aim of these studies was to gain insight into inter-individual differences in intracellular pharmacokinetics. Especially since some patients are resistant to treatment with azacitidine or decitabine or become resistant at some point [162].

Jansen et al. (2012) measured the intracellular aza-dCMP, aza-dCDP and azadCTP concentrations in PBMCs obtained from three patients diagnosed with MDS that were treated with decitabine $15 \mathrm{mg} / \mathrm{m}^{2}$ administered as a 4-h infusion repeated every $8 \mathrm{~h}$ for 3 days with a cycle duration of 6 weeks [159]. The highest concentrations were found for aza-dCTP. Aza-dCMP and aza-dCDP accounted for $2-29 \%$ and $2-21 \%$ of the total decitabine nucleotide concentration, respectively. The authors also searched for the possible presence of deaminated decitabine nucleotides, i.e. aza-dU nucleotides, but these were not observed. In contrast to plasma decitabine, intracellular aza-dCTP was found to accumulate during the treatment cycle of nine infusions. Two patients responded well to decitabine therapy. Remarkably, considerable aza-dCTP concentrations were 
measured in the PBMCs from these two patients, whereas only low levels were present in the third patient who did not respond [159].

Wang et al. (2013) measured the intracellular aza-dCTP concentrations in bone marrow cells and PBMCs from seven AML patients that were treated with decitabine $20 \mathrm{mg} / \mathrm{m}^{2}$ infused in $1 \mathrm{~h}$ for 10 days [160]. Samples were collected on days 1 and 5 within $3 \mathrm{~h}$ after completion of the decitabine infusion. Other than in the study by Jansen et al., repeated dosing did not result in accumulation of intracellular aza-dCTP during the treatment cycle. Aza-dCTP levels were found to be relatively stable from day 1 to 5 . In line with the study by Jansen et al., higher intracellular aza-dCTP levels appeared to be associated with clinical response also in this study. Patients who eventually achieved a complete response had higher aza-dCTP levels in both bone marrow and PBMCs than those who failed to respond to the treatment. However, also in this explorative study, the number of patients was too small to draw definitive conclusions [160].

Following these two studies with decitabine triphosphate (aza-dCTP), we developed an assay to quantify azacitidine triphosphate (aza-CTP) in PBMCs [161]. To quantify aza-CTP we had to use high-resolution mass spectrometry, because regular LC-MS/MS could not distinguish between aza-CTP and some of the endogenous nucleotides. Interference by highly abundant endogenous nucleotides proved to be more complex for aza-CTP than it had been for azadCTP. The developed assay was applied to quantify aza-CTP in PBMCs of two patients diagnosed with MDS who were treated with azacitidine $75 \mathrm{mg} /$ $\mathrm{m}^{2}$ subcutaneously for 7 days, every 4 weeks. The aza-CTP concentrations measured were about 10-25 times higher than the aza-dCTP concentrations presented by Jansen et al. and Wang et al. (Table 2) [159,160]. To our knowledge, this was the first time that aza-CTP concentrations were measured in cell material from patients treated with azacitidine [161].

We have not found any other studies that determined the intracellular nucleotide concentrations after azacitidine therapy. Nevertheless, the recent study by Unnikrishnan et al. (2018) is very interesting to mention in this light [162]. This study examined the successive steps in the pharmacology of azacitidine in eight patients diagnosed with MDS or CMML, and showed that responders to azacitidine therapy demonstrated more aza-dC incorporation into DNA and 
more DNA demethylation than non-responders. Unfortunately, the intracellular aza-CTP and aza-dCTP concentrations were not measured in this study. Instead, all intracellular nucleotides were enzymatically dephosphorylated during sample preparation, after which the total intracellular aza-C and aza-dC concentrations were measured. Interestingly, the non-responders showing minimal DNA incorporation and minimal DNA demethylation showed high intracellular aza- $\mathrm{C}$ and aza-dC concentrations (after dephosphorylation) as well as high aza-C incorporation into RNA. This means that the low aza-dC DNA incorporation could not be explained by limited cellular uptake and that the intracellular metabolism (at least the RNA pathway) was also intact. The authors suggest that aza-dC incorporation into DNA might be low in patients if an increased proportion of the bone marrow cells is quiescent and not undergoing DNA replication [162]. It would be interesting to measure the aza-CTP and aza-dCTP concentrations in these patients and examine if they correlate with DNA incorporation and response.

\section{Azacitidine and decitabine in conclusion}

The number of intracellular PK studies performed for azacitidine and decitabine is limited. Nevertheless, the two exploratory studies conducted so far for decitabine both suggest a relationship between intracellular aza-dCTP levels and clinical response to decitabine therapy. This relationship should be further investigated in larger numbers of patients and should also be examined for azacitidine. If a relationship between intracellular triphosphate levels and response is confirmed, the intracellular levels of aza-CTP or aza-dCTP can serve as a biomarker for early identification of individuals who are unlikely to respond to treatment with azacitidine or decitabine. Further studies should show whether these patients would benefit from a higher dose of azacitidine or decitabine or whether they should switch to other therapies. 


\section{DISCUSSION}

\section{Differences between pyrimidine analogues}

Although the intracellular nucleotide concentrations during cytarabine and gemcitabine treatment have been extensively studied, the intracellular nucleotide concentrations during treatment with 5-FU, capecitabine, azacitidine, and decitabine have only been investigated to a limited extent. This is remarkable. For a long time, there were no suitable bio-analytical assays for quantifying the active nucleotides of the latter pyrimidine analogues. This prompted us to develop these assays $[44,159,161]$. We hope that the developed assays will be widely used to gain more insight into the intracellular pharmacokinetics of the less extensively studied pyrimidine analogues.

The intracellular nucleotide concentrations measured in different studies are sometimes difficult to compare because different units were used (Table 2). The intracellular concentrations were reported in pmol/106 PBMCs, ng/ mg protein or were converted to $\mu \mathrm{M}$ based on cell volume. In this review, data were presented as reported in the original publications. Units were not converted, because not all required information, for instance the measured cell volume, was published in the original articles. Nevertheless, Table 2 shows that the intracellular nucleotide concentrations during treatments with 5-FU, capecitabine, azacitidine, and decitabine (in the dosages studied) were very low compared with the intracellular nucleotide concentrations during treatment with cytarabine or gemcitabine.

\section{Relationship between the intracellular nucleotide concentrations and the antineoplastic effect}

Based on pharmacology, a relationship is expected between the intracellular concentrations of the active nucleotides and the therapeutic effect of pyrimidine analogues. This relationship has been demonstrated in in vitro studies and animal studies [117]. However, not many clinical studies have investigated the relationship between intracellular nucleotide concentrations and the clinical outcome in patients. Only for cytarabine, a relationship was demonstrated between the intracellular triphosphate concentrations in leukemic cells and the 
response rate in patients with AML [87]. Future clinical studies should show, for the other pyrimidine analogues, whether there is a relationship between the intracellular nucleotide concentrations and the therapeutic effect in patients.

\section{Optimization of dosing regimens}

Research that examined the intracellular pharmacokinetics of cytarabine and gemcitabine focused primarily on the saturation aspect of the intracellular triphosphate formation $[60,61,97,110]$. Attempts to improve the dosing regimen of gemcitabine were aimed at maximizing the intracellular gemcitabine triphosphate concentrations [113-116,118,121,124]. However, if the dose is based on the maximum tolerated dose and the mechanisms underlying the therapeutic effect and toxicity are exactly the same, this strategy does not make sense. Efficient administration of gemcitabine, aimed at maximum triphosphate formation, also means that less gemcitabine can be administered before dose limiting toxicities are achieved [125-127]. In all cases, the dosage is limited by the 'maximum tolerated intracellular triphosphate exposure'.

If we really want to improve the dosing regimens of pyrimidine analogues, we will have to examine if there are areas where the mechanism of action and the mechanism of toxicity are not completely parallel. One could think of differences in cellular uptake or phosphorylation capacity between healthy cells and tumour cells. It is also useful to investigate the effects of high plasma peaks which far exceed plasma concentrations that saturate the intracellular triphosphate formation. What happens to the excess of pyrimidine analogue that is not phosphorylated intracellularly? What is, for instance, the effect of deamination products such as ara- $U$ and dFdU? A recent study in which we investigated the intracellular pharmacokinetics of gemcitabine showed that dFdU was only phosphorylated to a very limited extent in PBMCs [131]. This suggests that the contribution of dFdU to the cytotoxic effect of gemcitabine is limited.

\section{Inter-individual differences}

For all pyrimidine analogues, a linear relationship was found between the dose and the plasma concentration [15,59-62,97,138]. However, no correlation was found between the plasma concentration and the intracellular nucleotide 
concentration $[45,59,110]$. The concentration-time curves for the intracellular nucleotides showed considerable inter-individual variation. This is not unexpected as many different proteins are involved in cellular uptake and the extensive intracellular metabolism of the pyrimidine analogues. The activity of all these membrane transporters and enzymes might vary from patient to patient.

Given the considerable inter-individual differences observed for the intracellular nucleotide concentrations, the question arises whether pyrimidine analogue therapy should be more individualized. Future research should show which intracellular nucleotide concentrations are worth pursuing and whether dose individualization is useful to achieve these concentrations. In addition, the possibility of using the intracellular nucleotide concentration as a predictive marker for the therapeutic effect might be investigated. In this way, potential non-responders, due to inadequate intracellular metabolism, could be recognized early. 


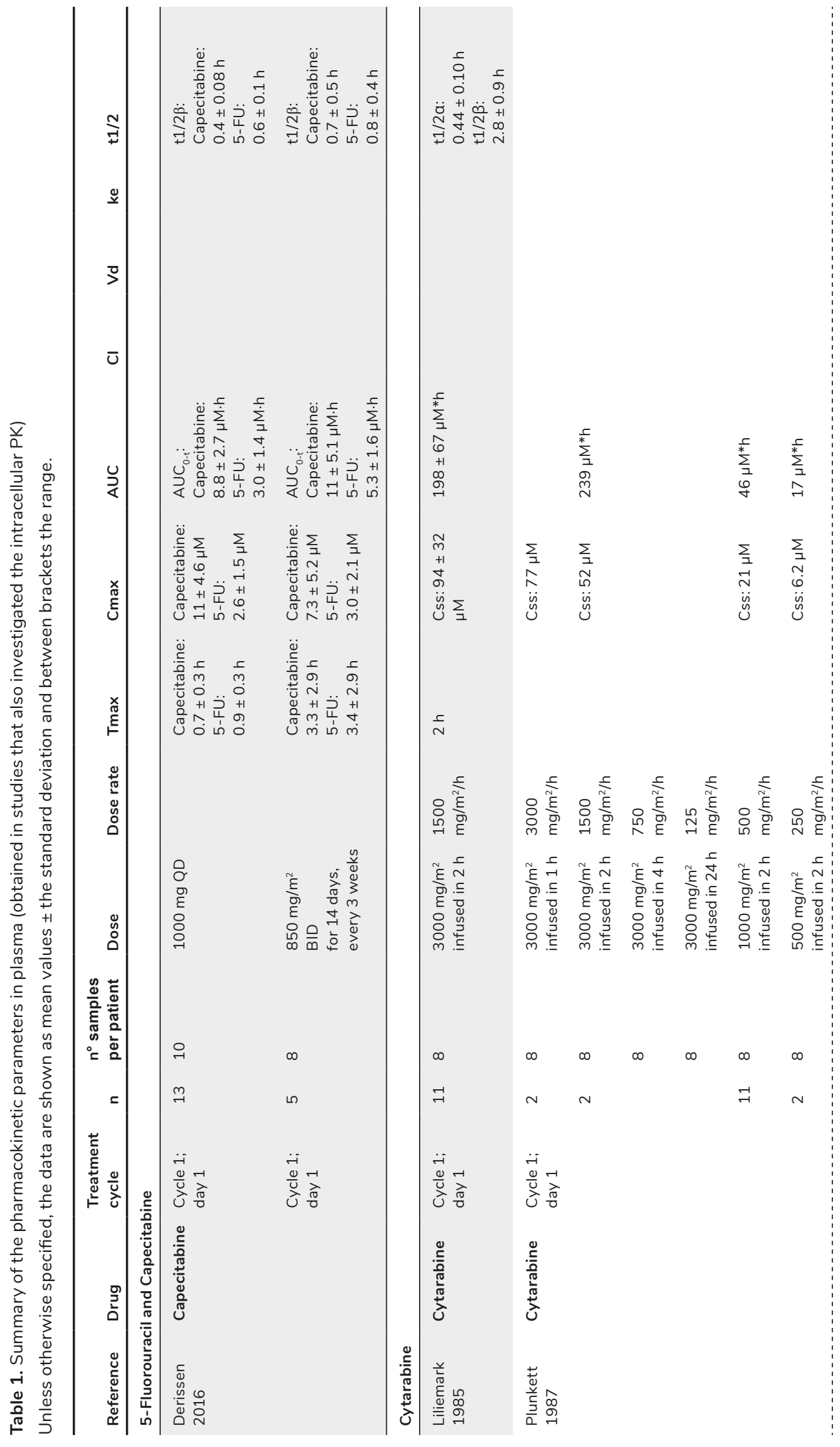




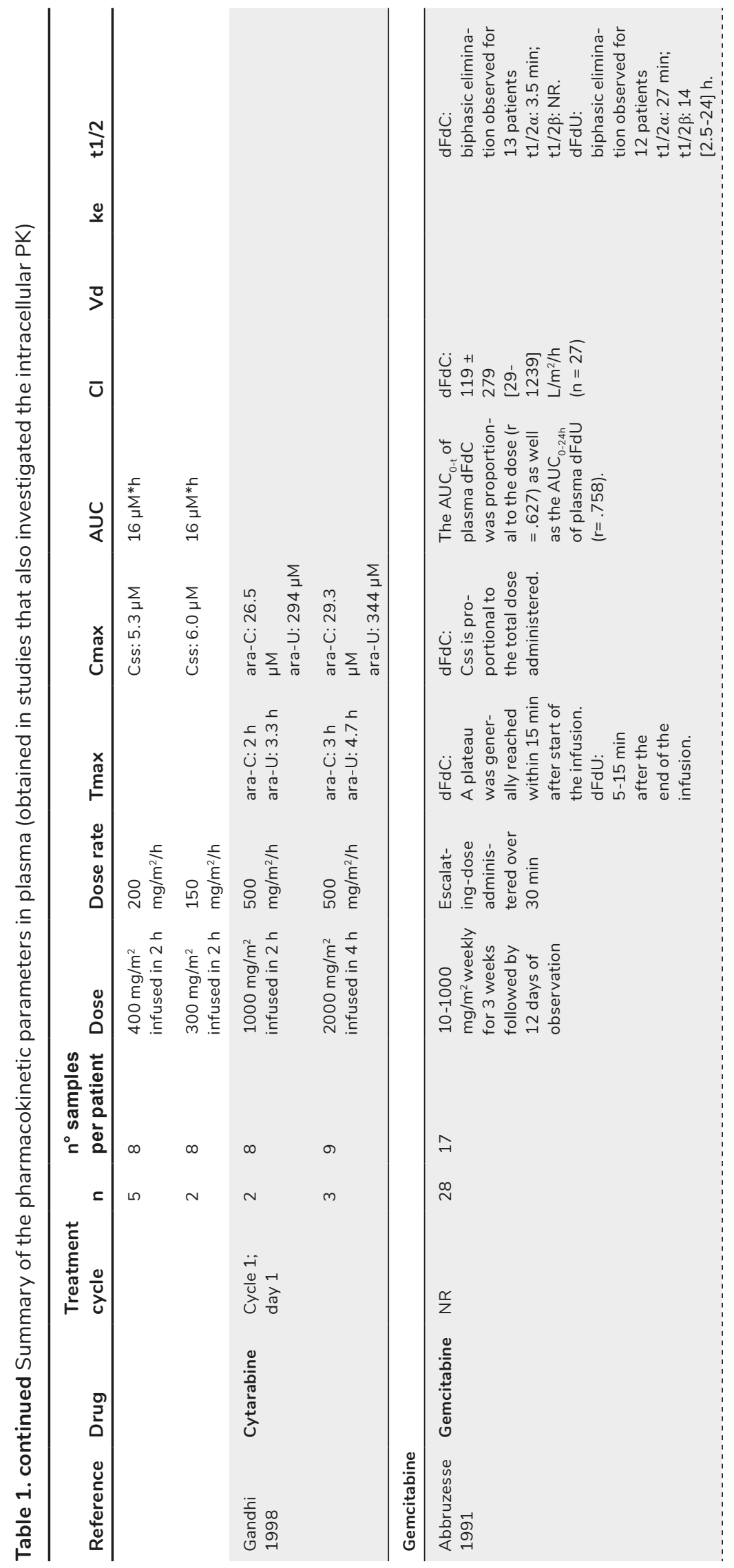




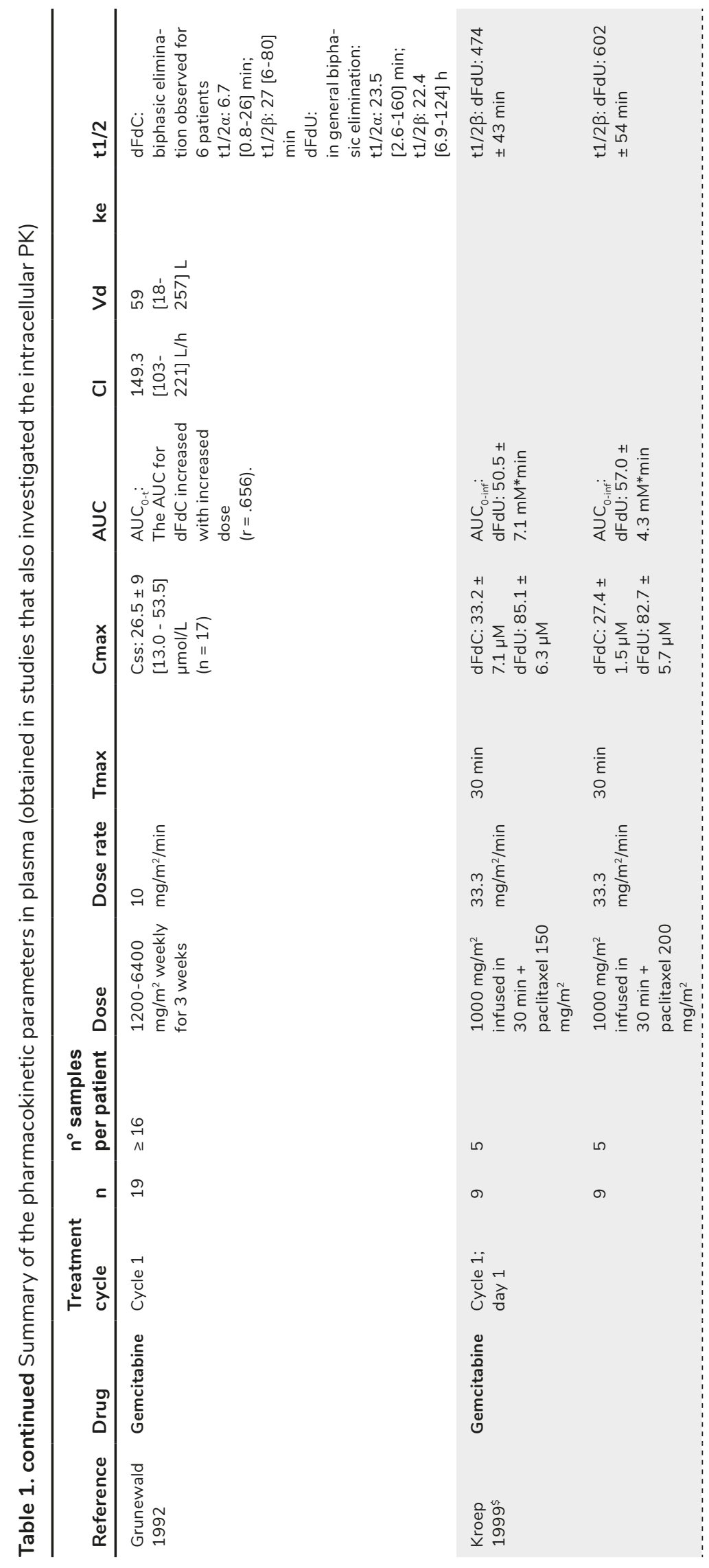




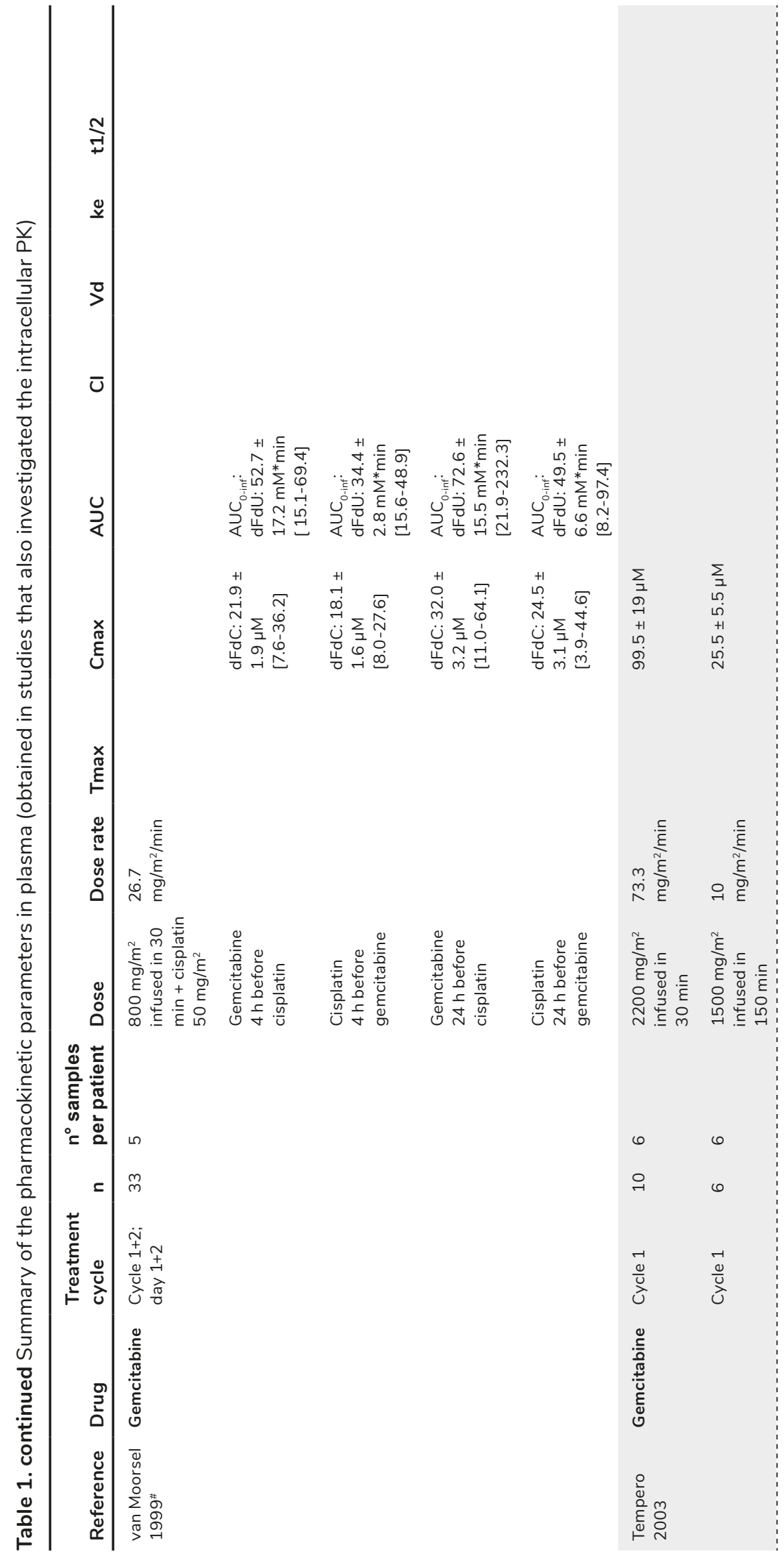




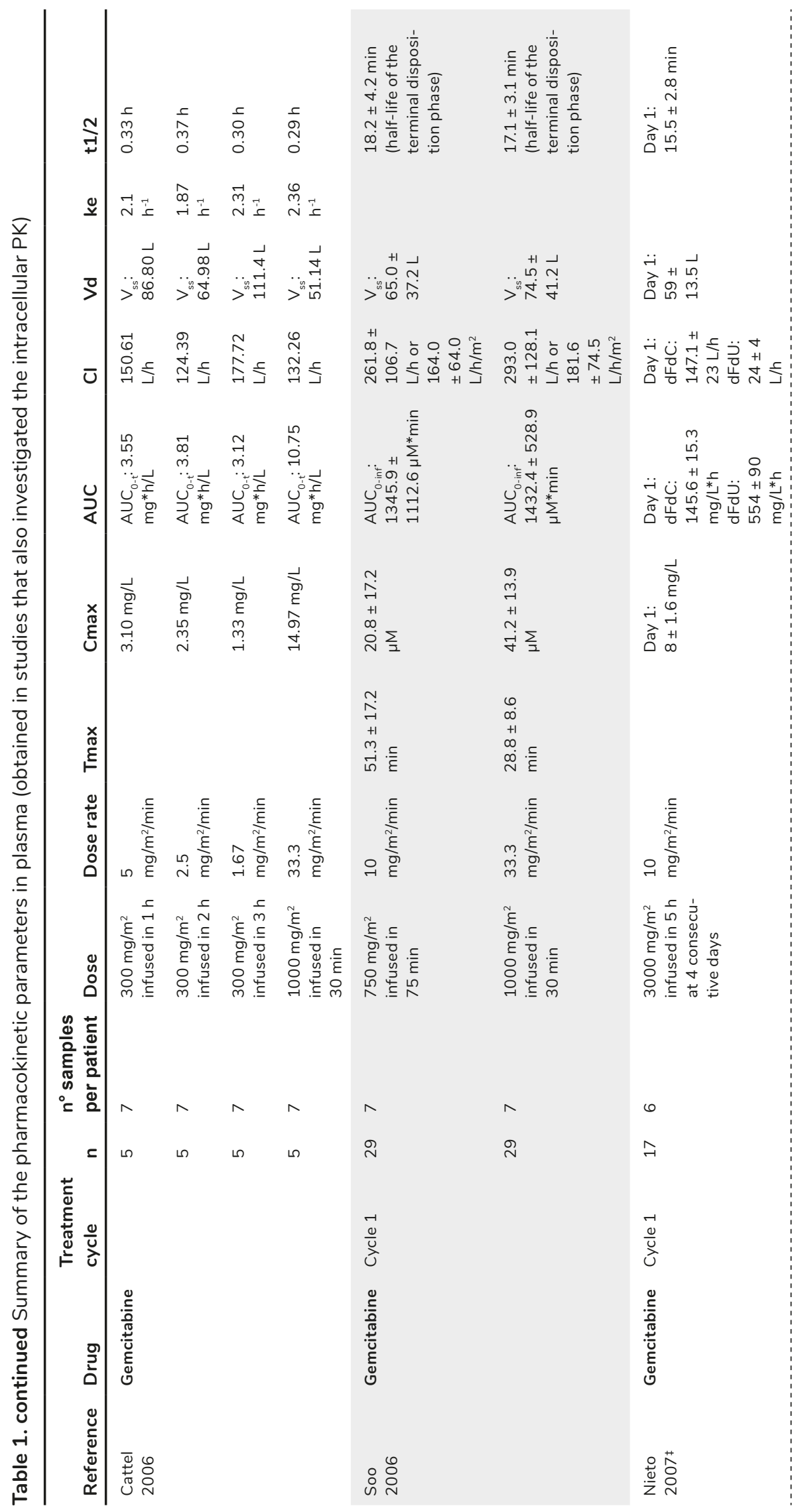




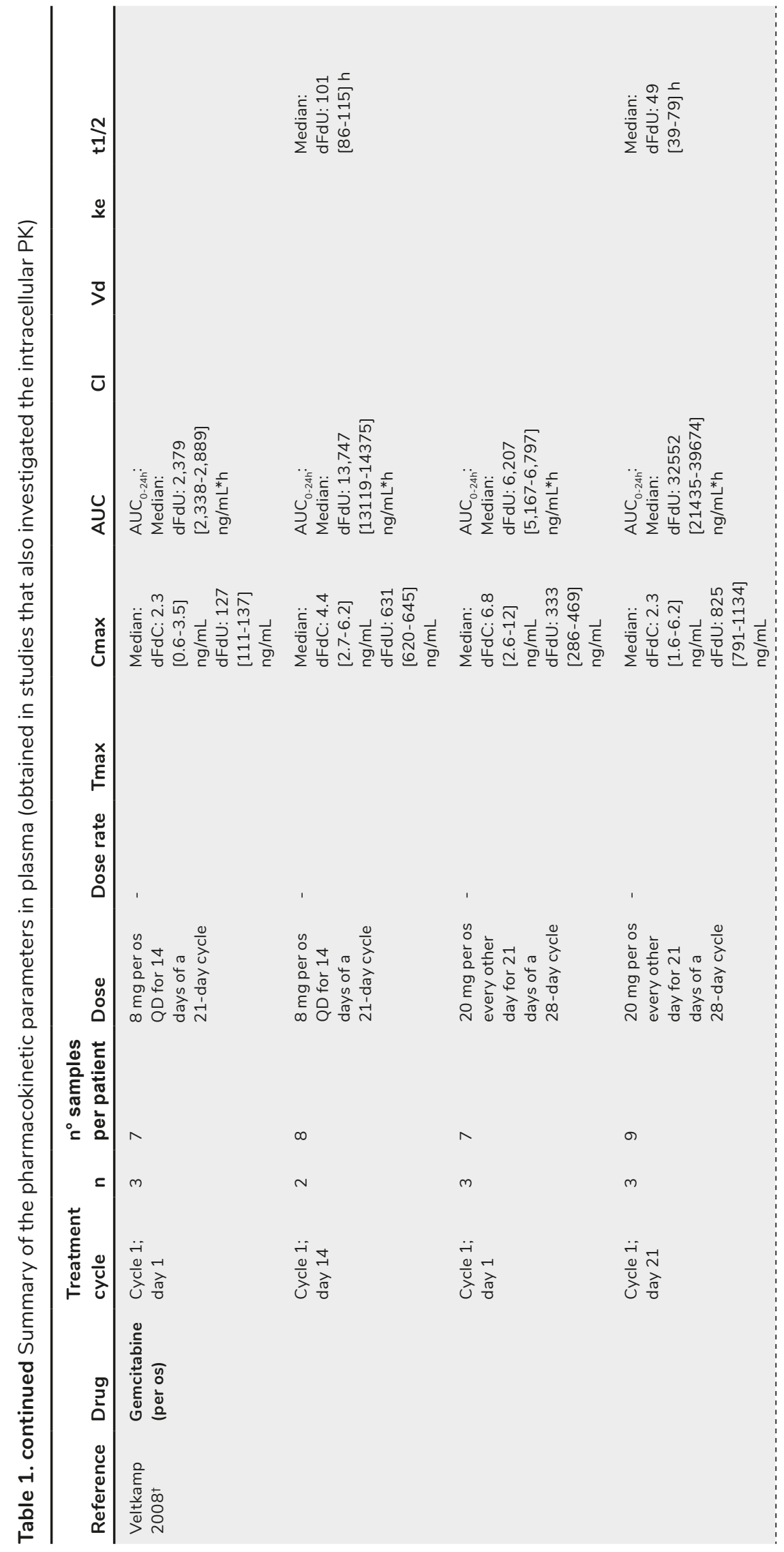




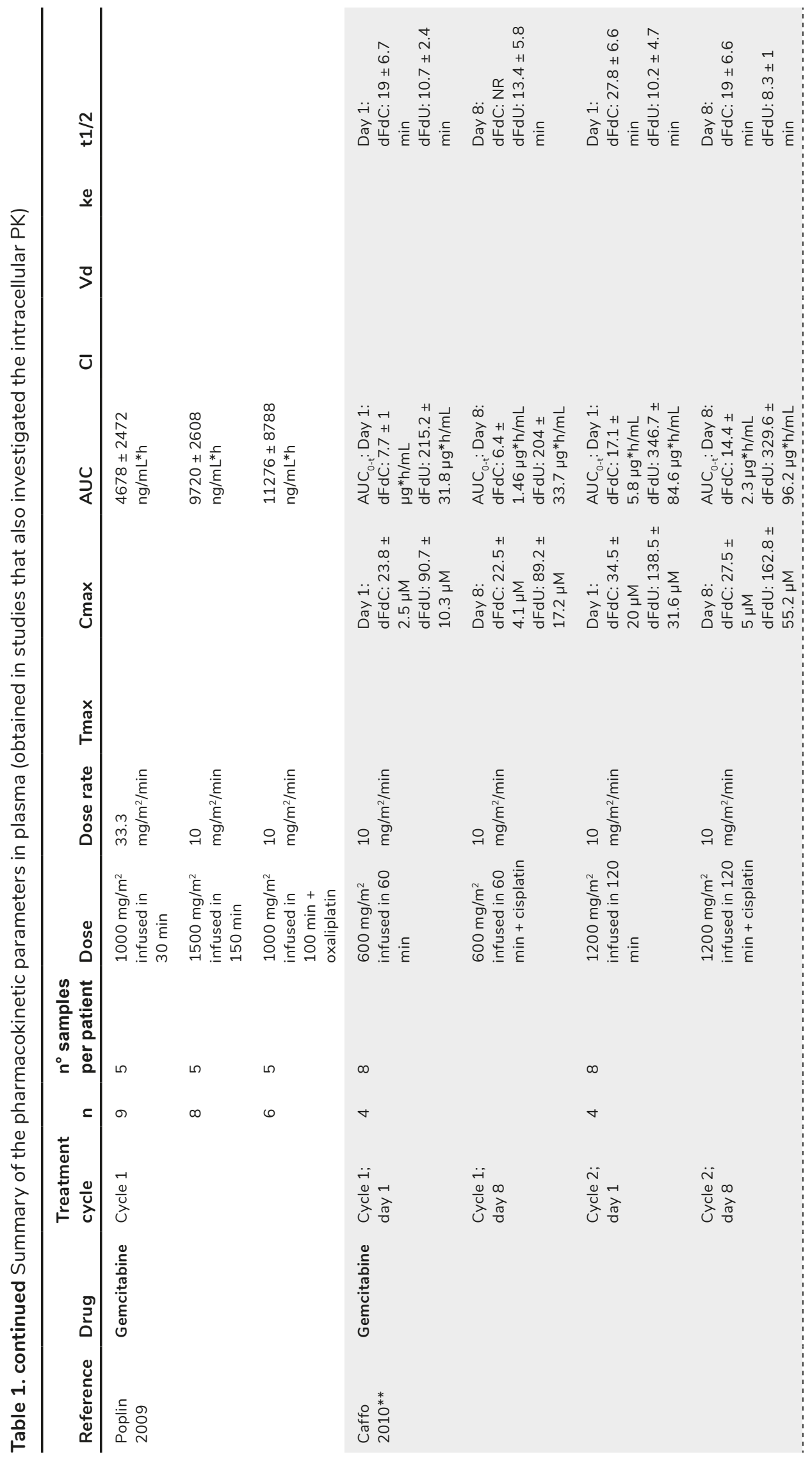




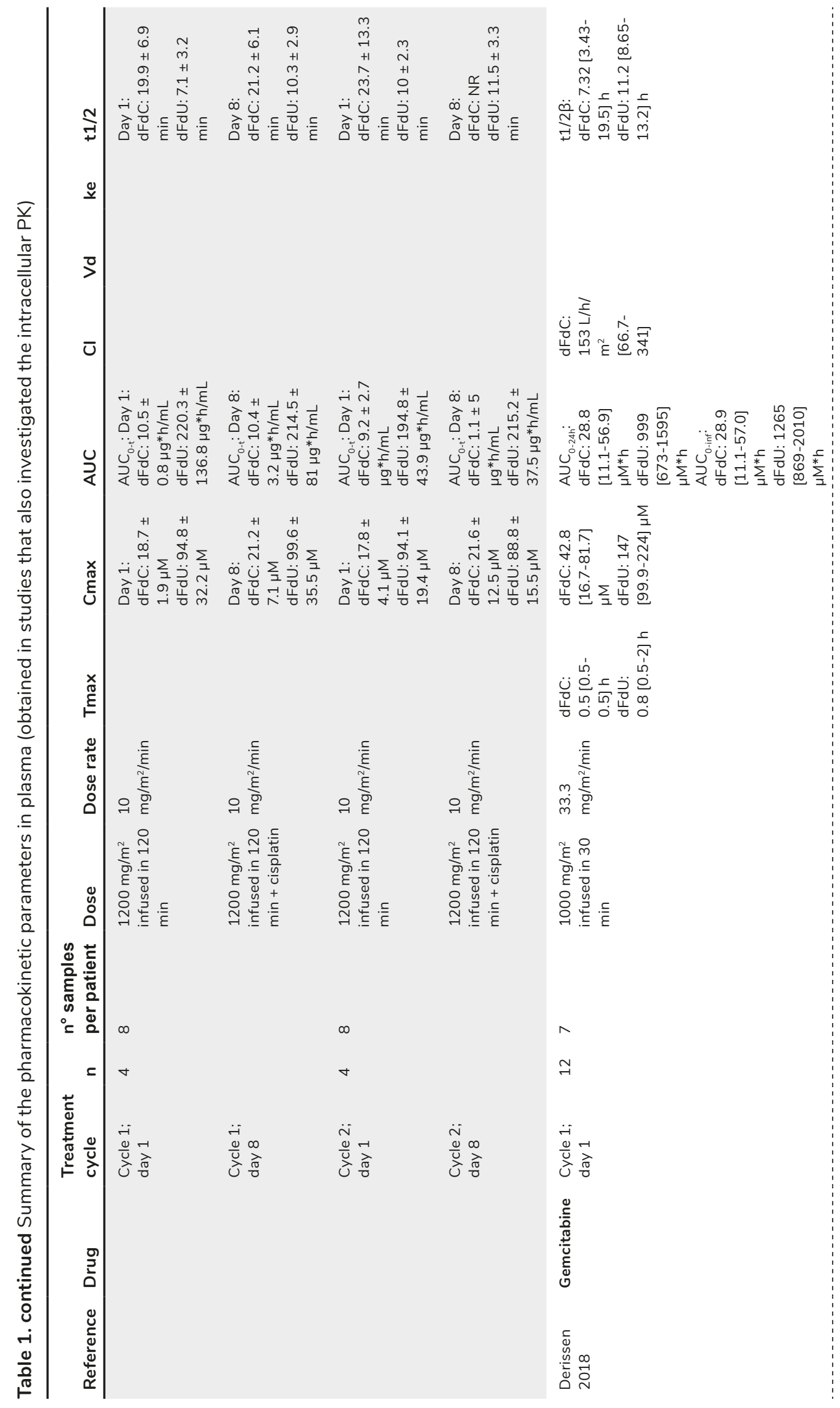




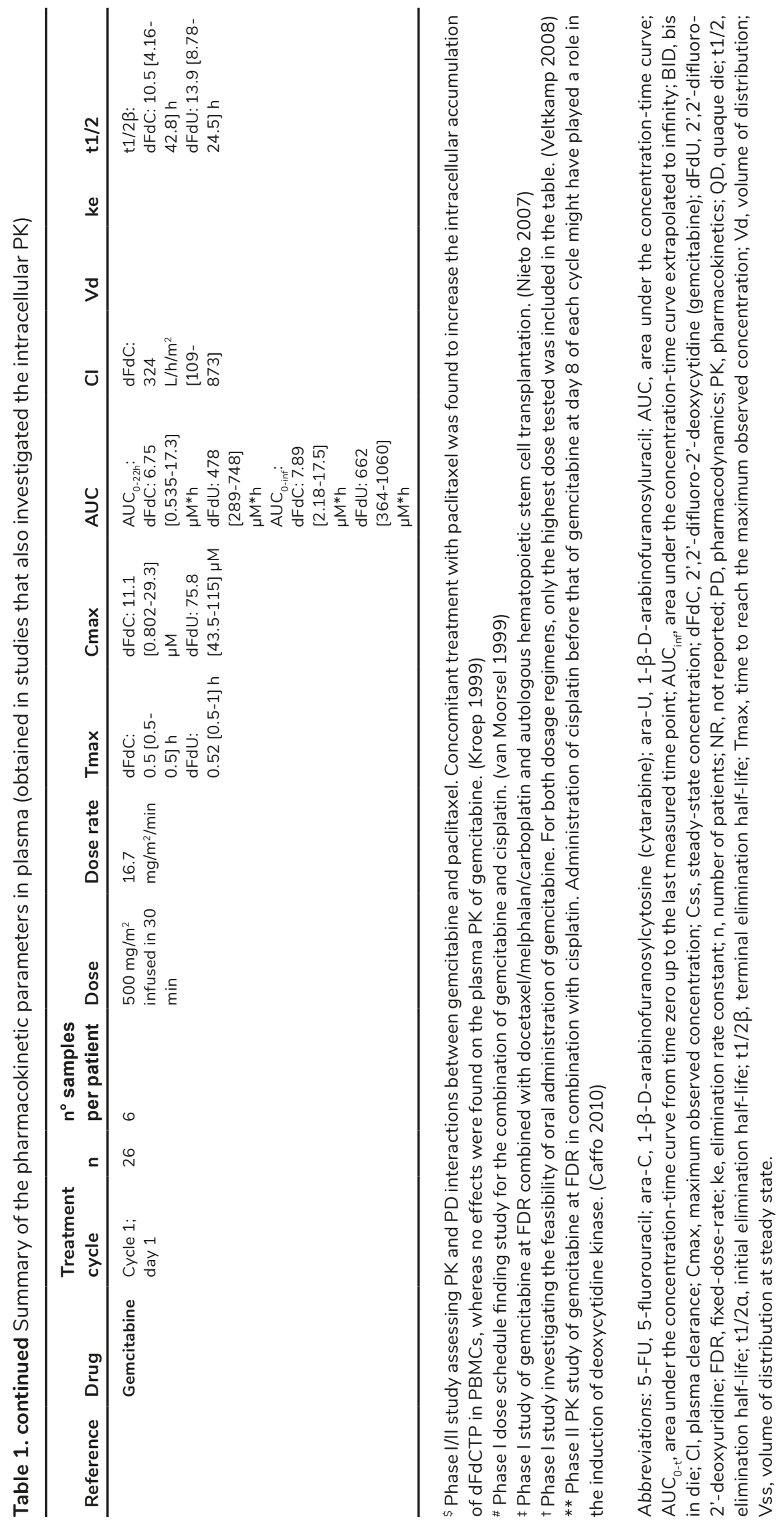




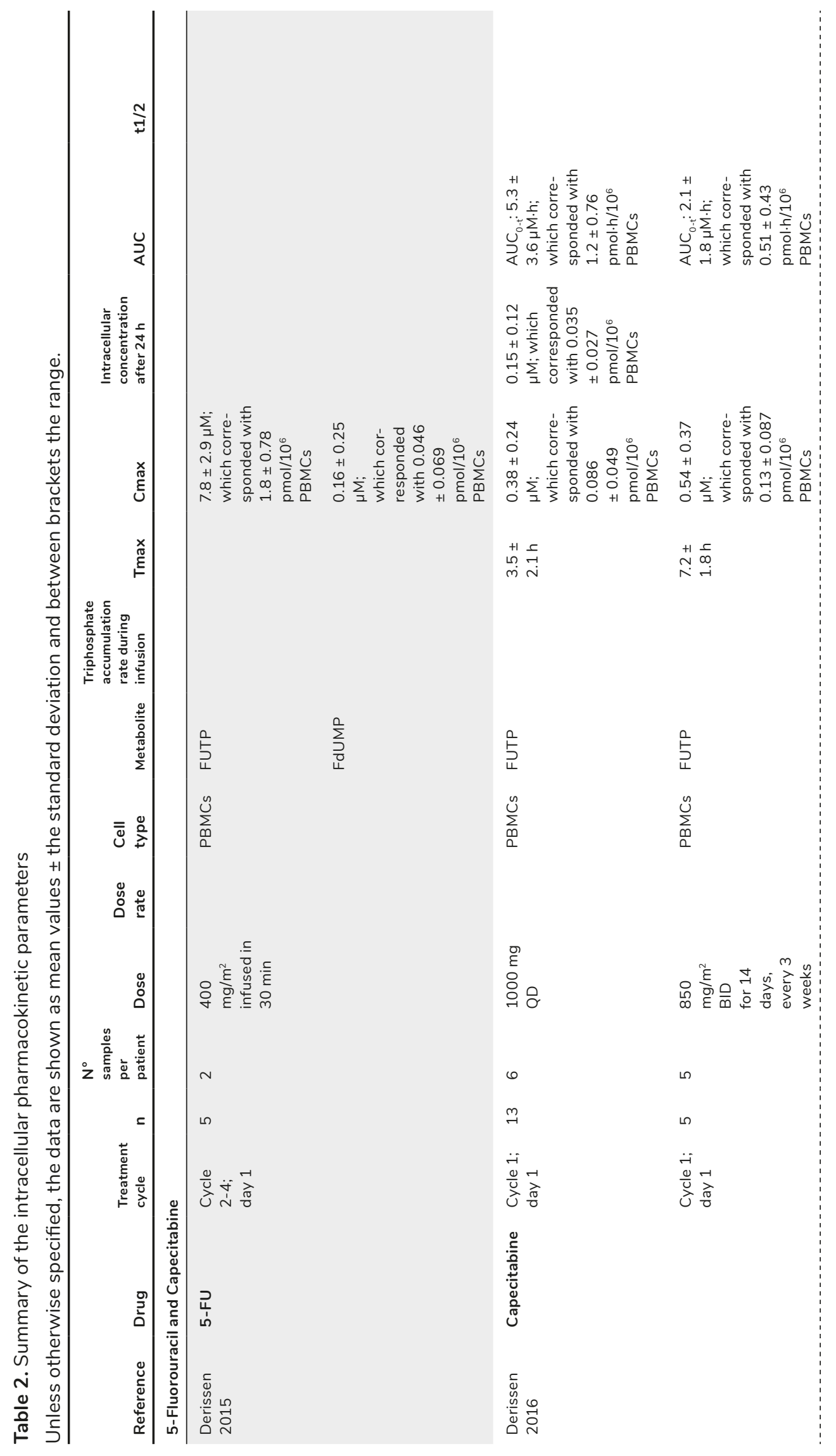




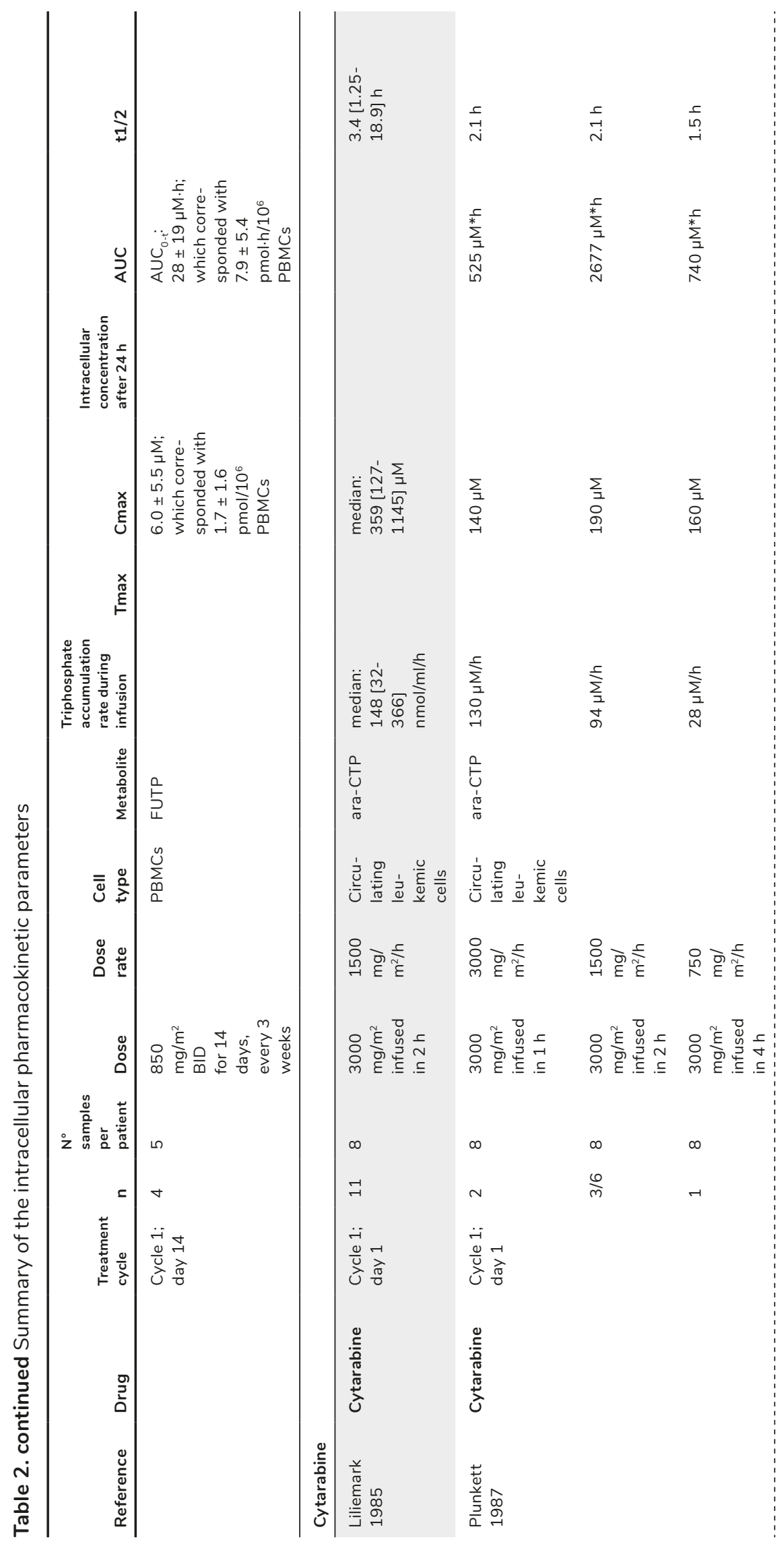




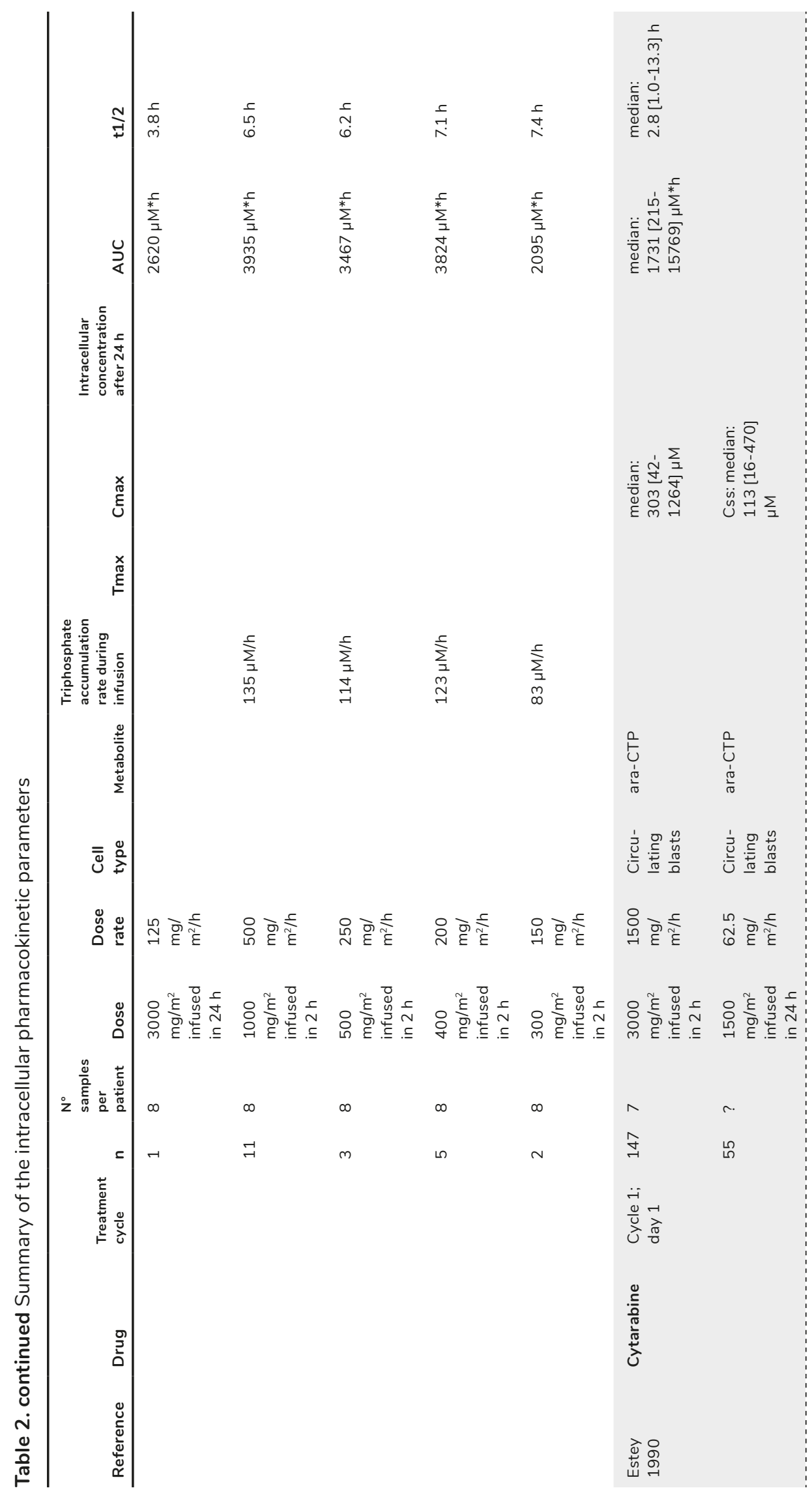




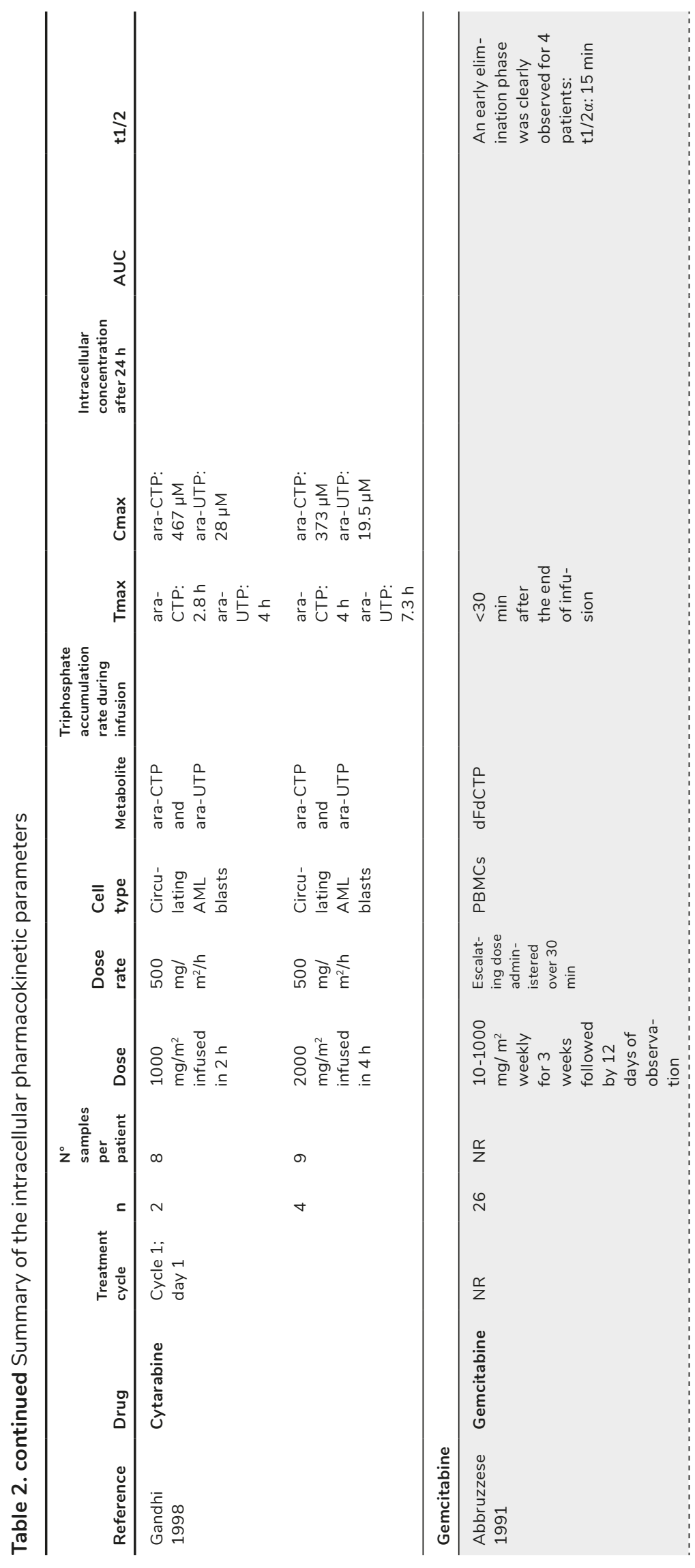




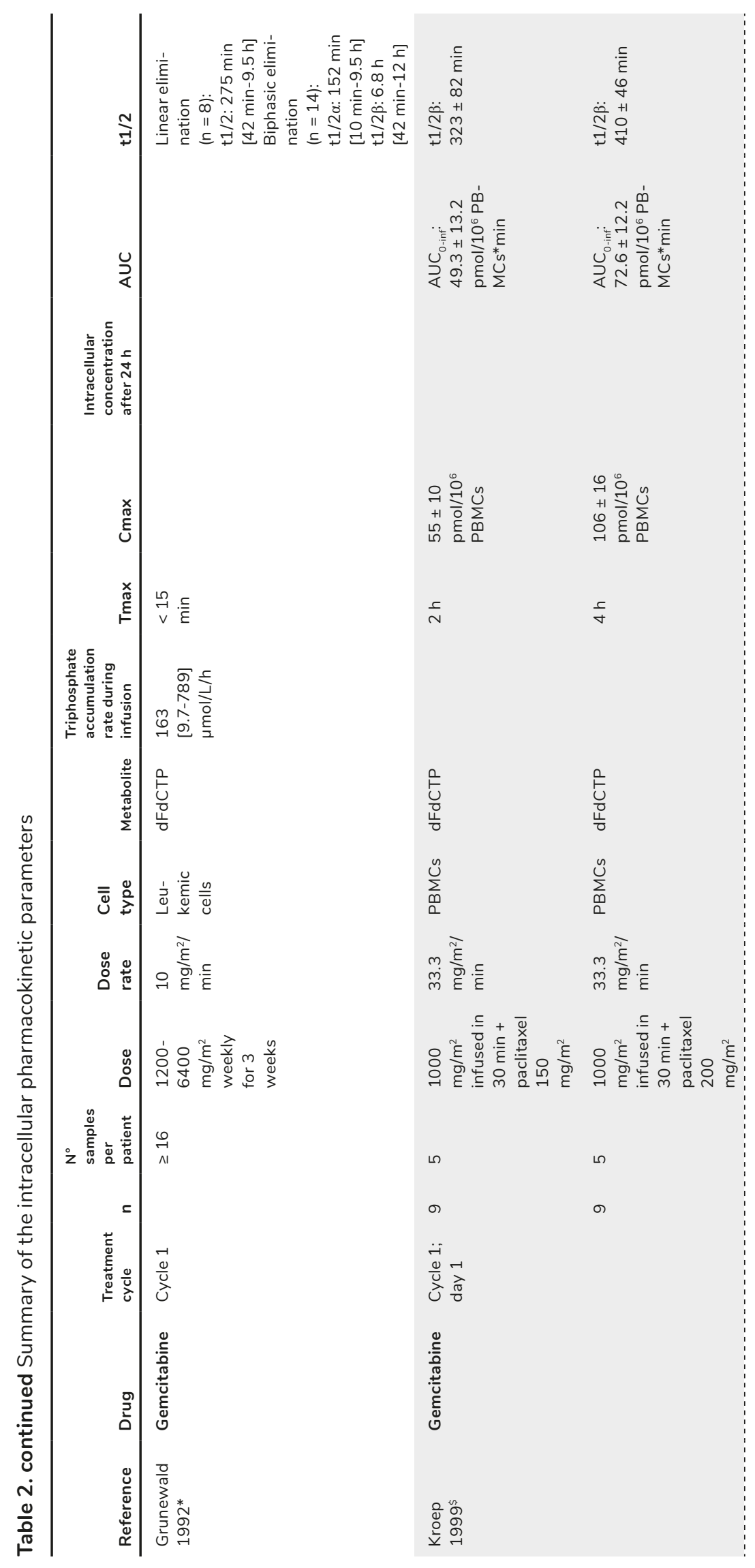




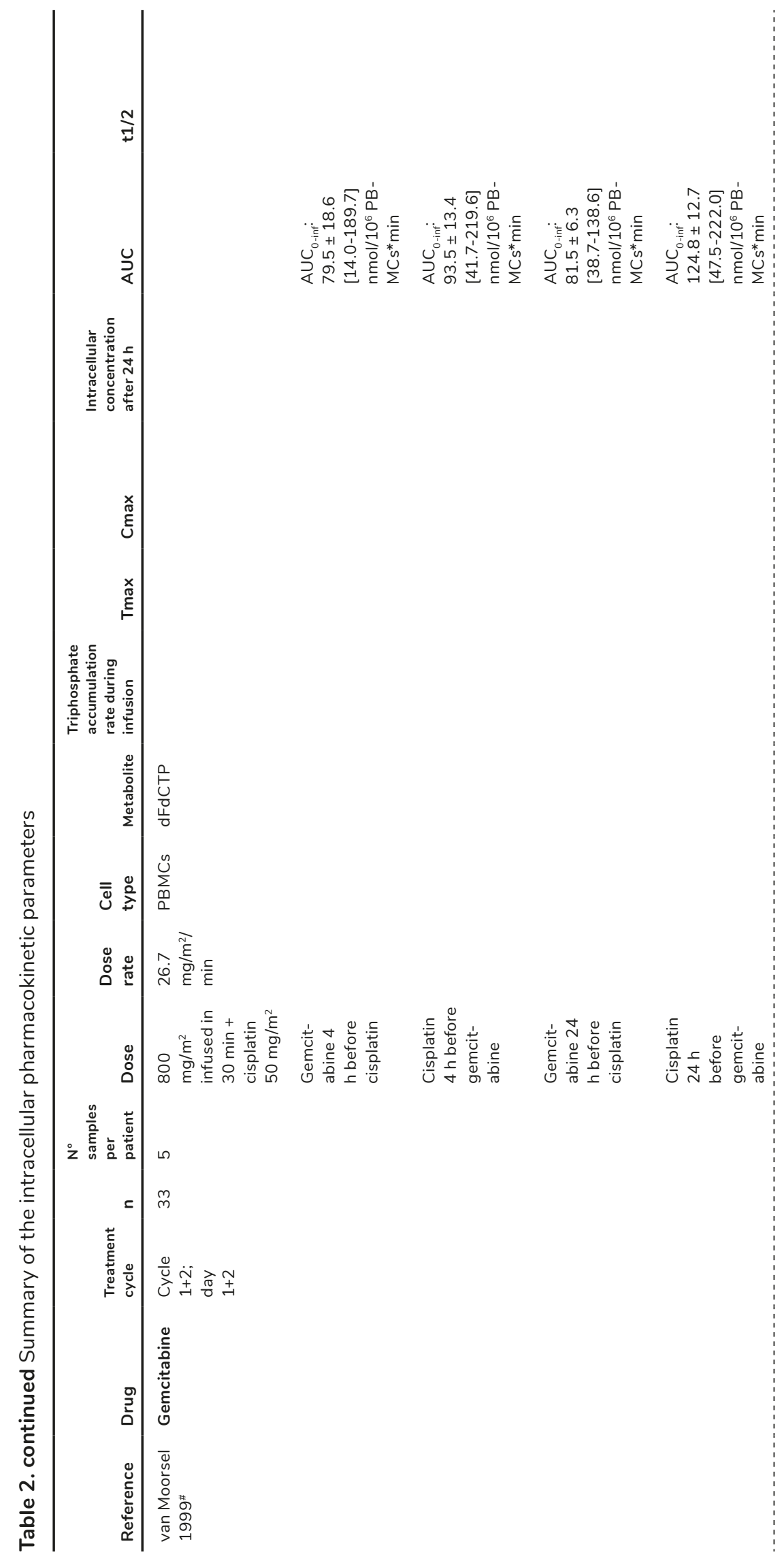




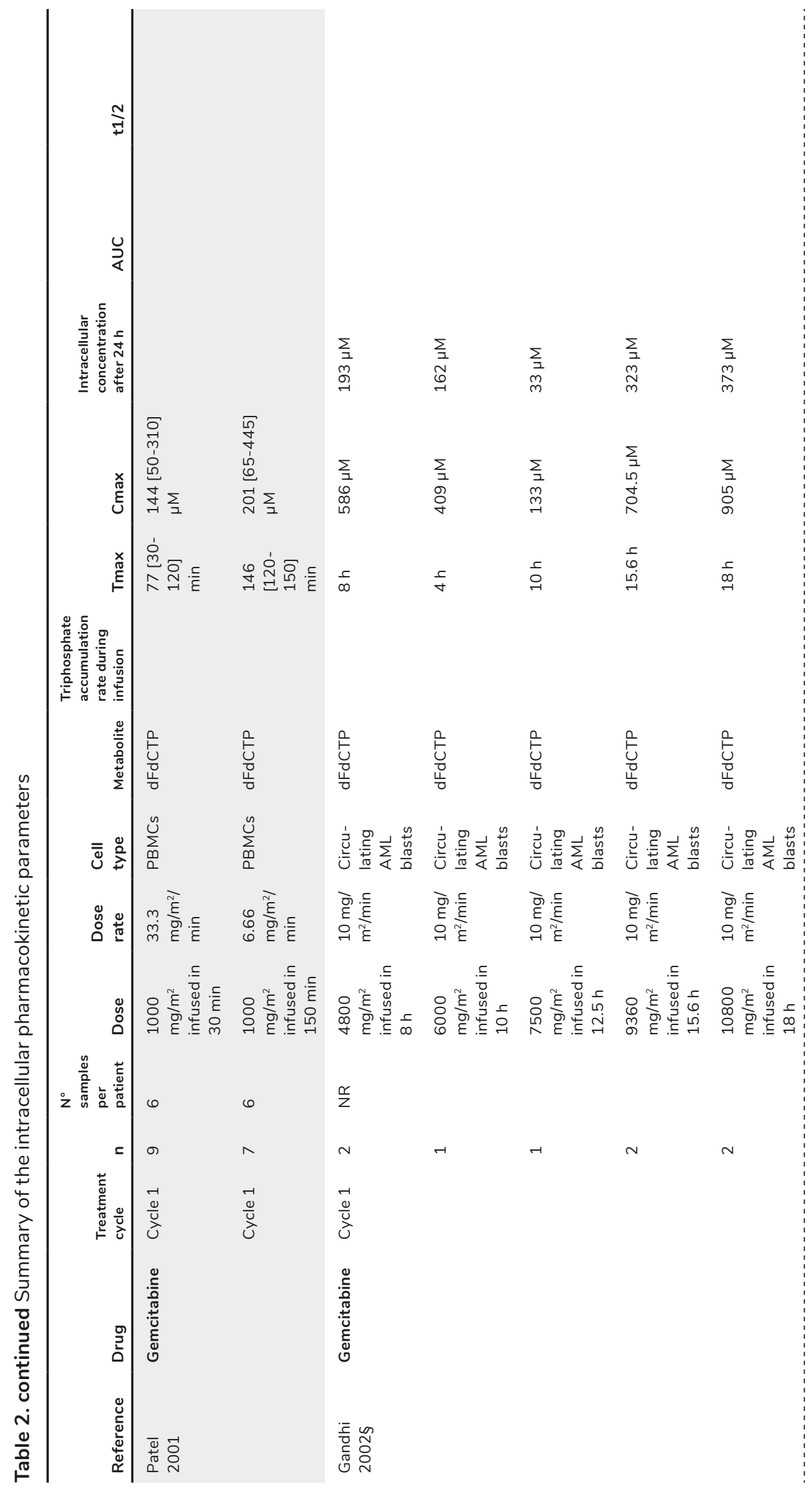




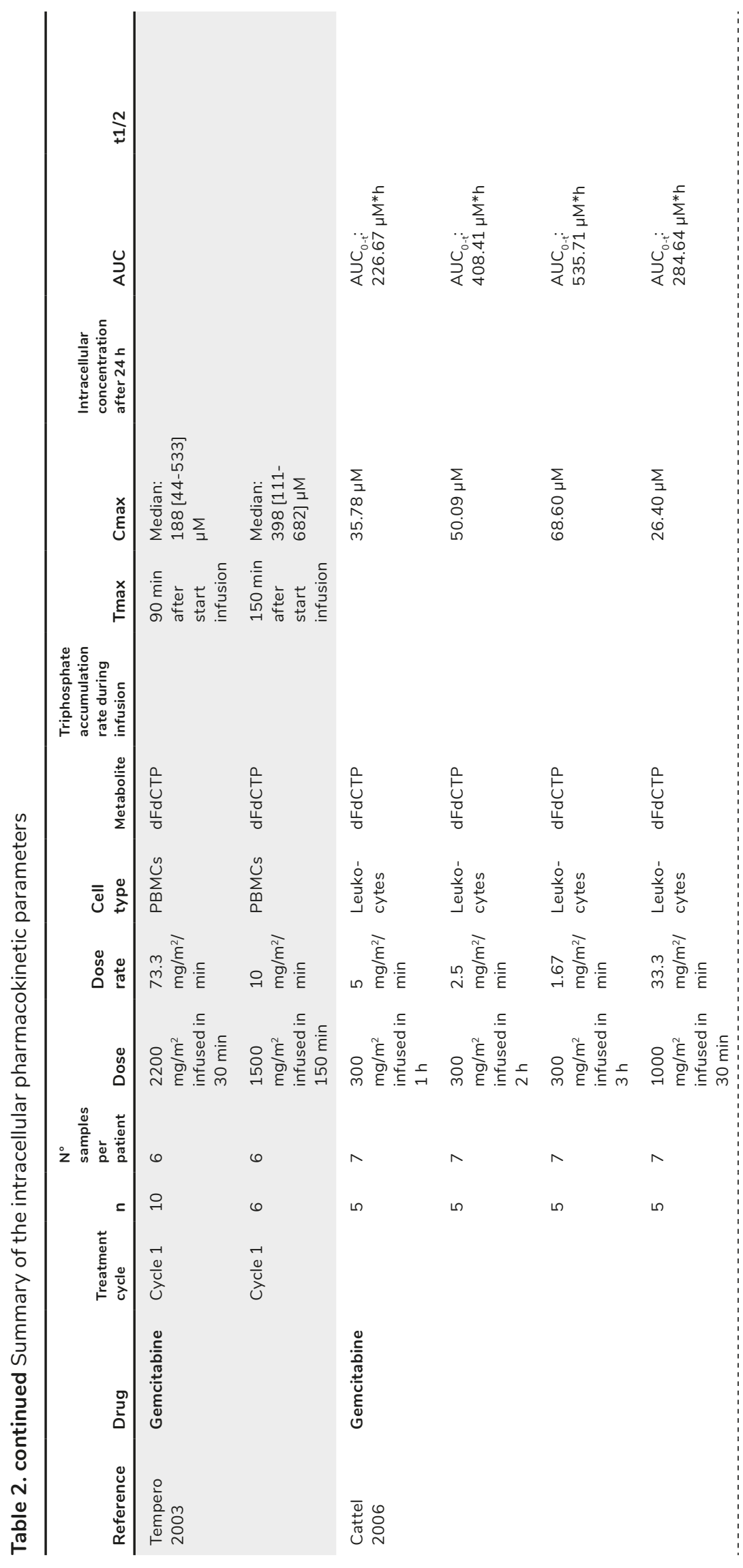




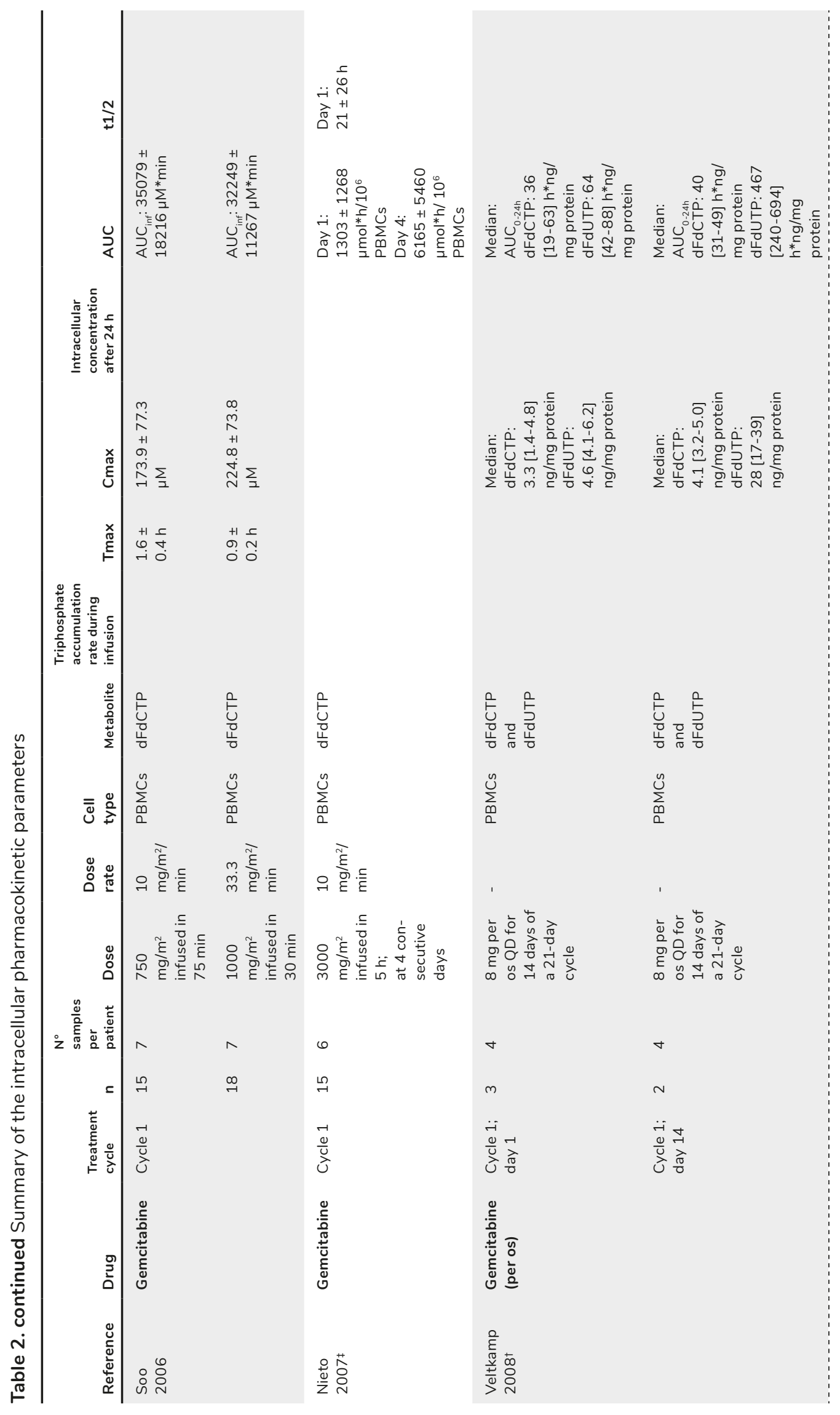




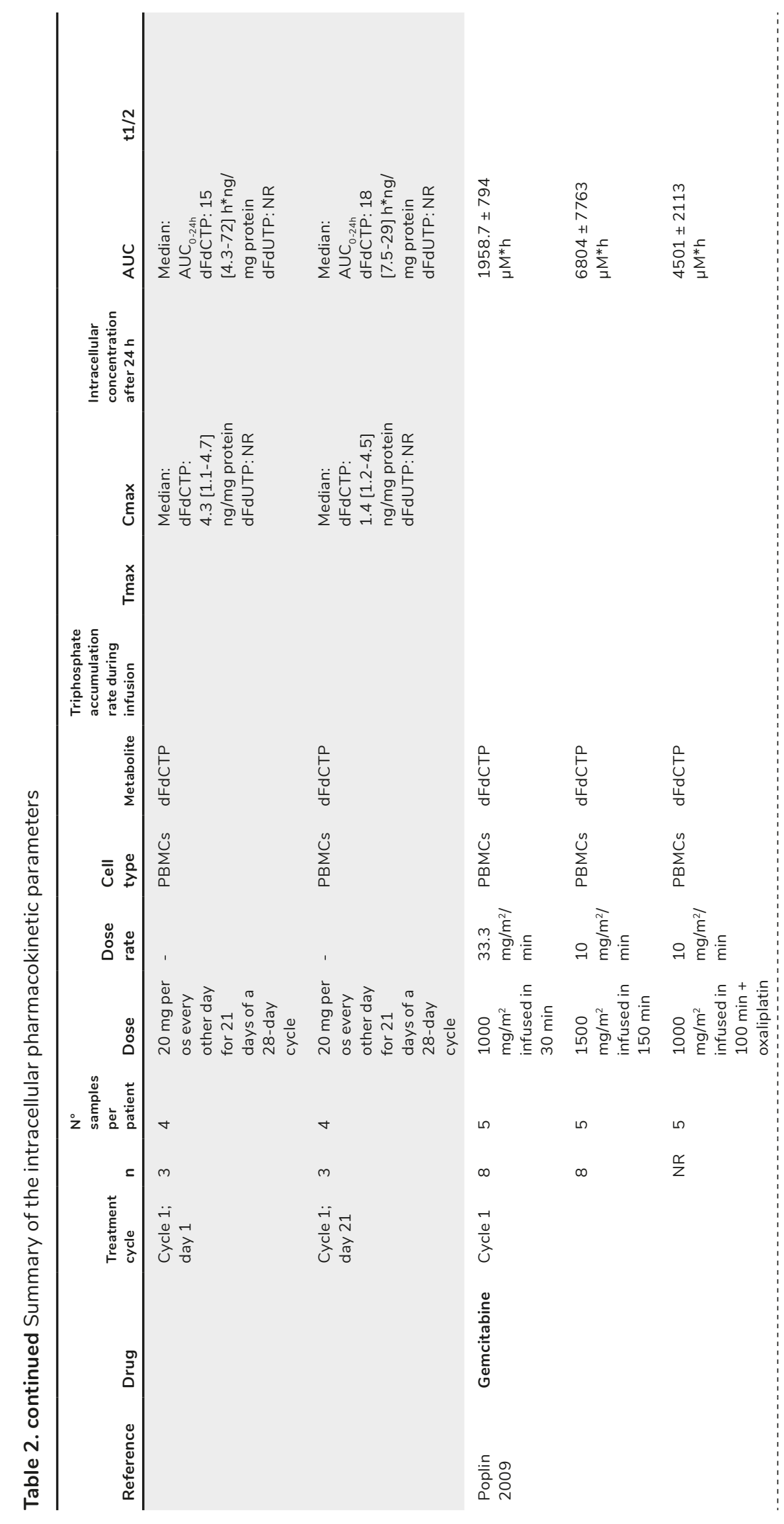




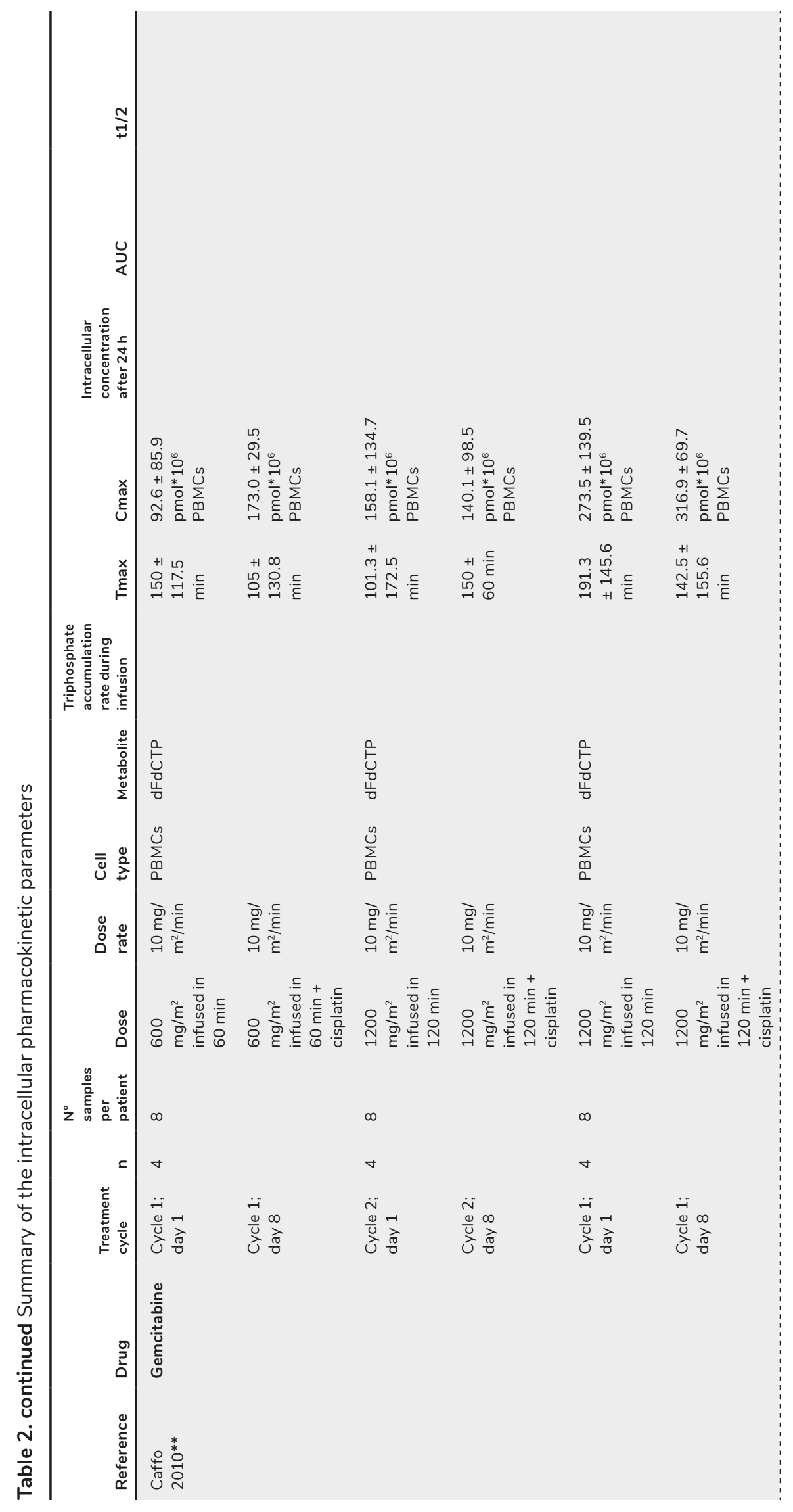




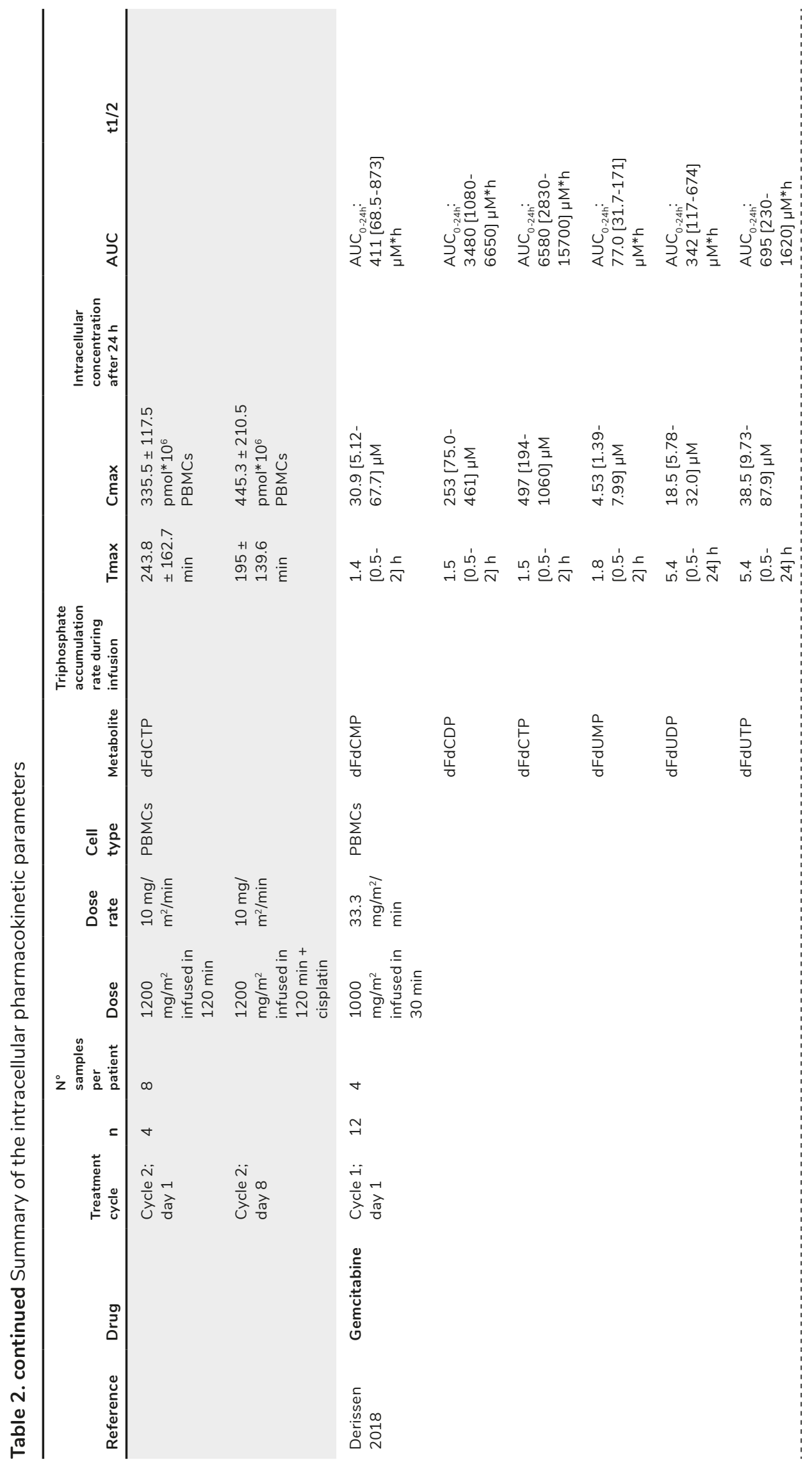




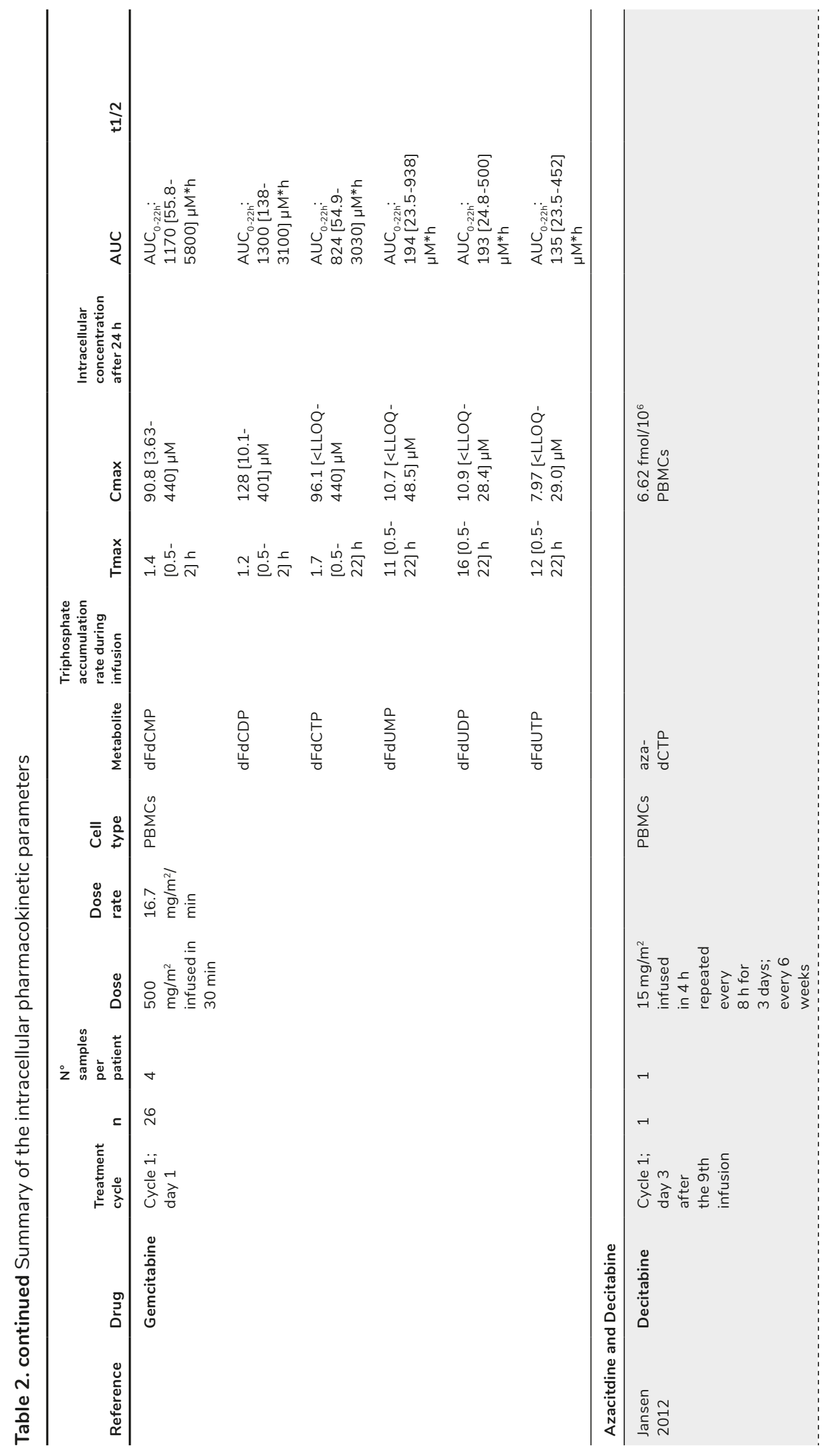




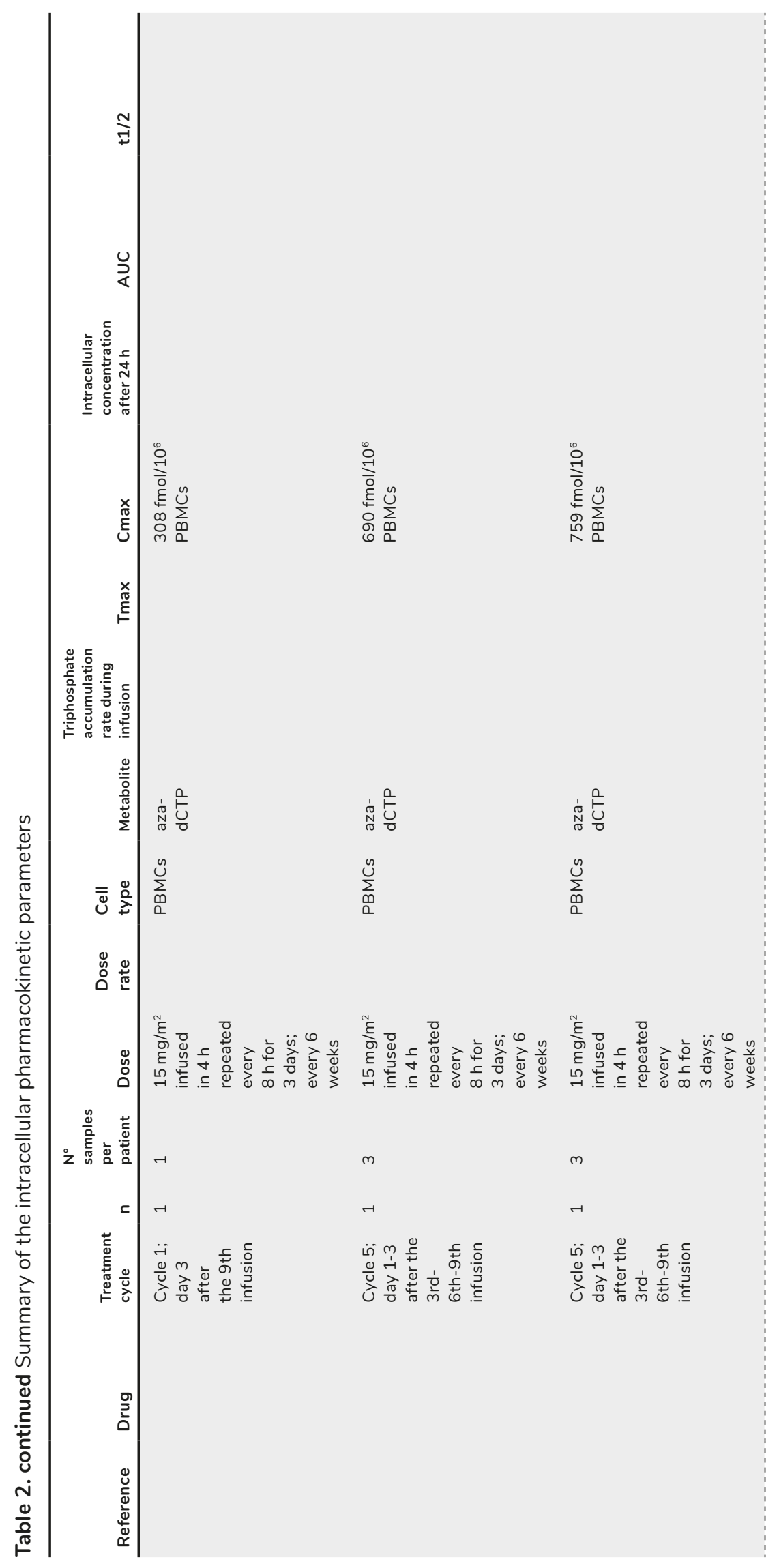




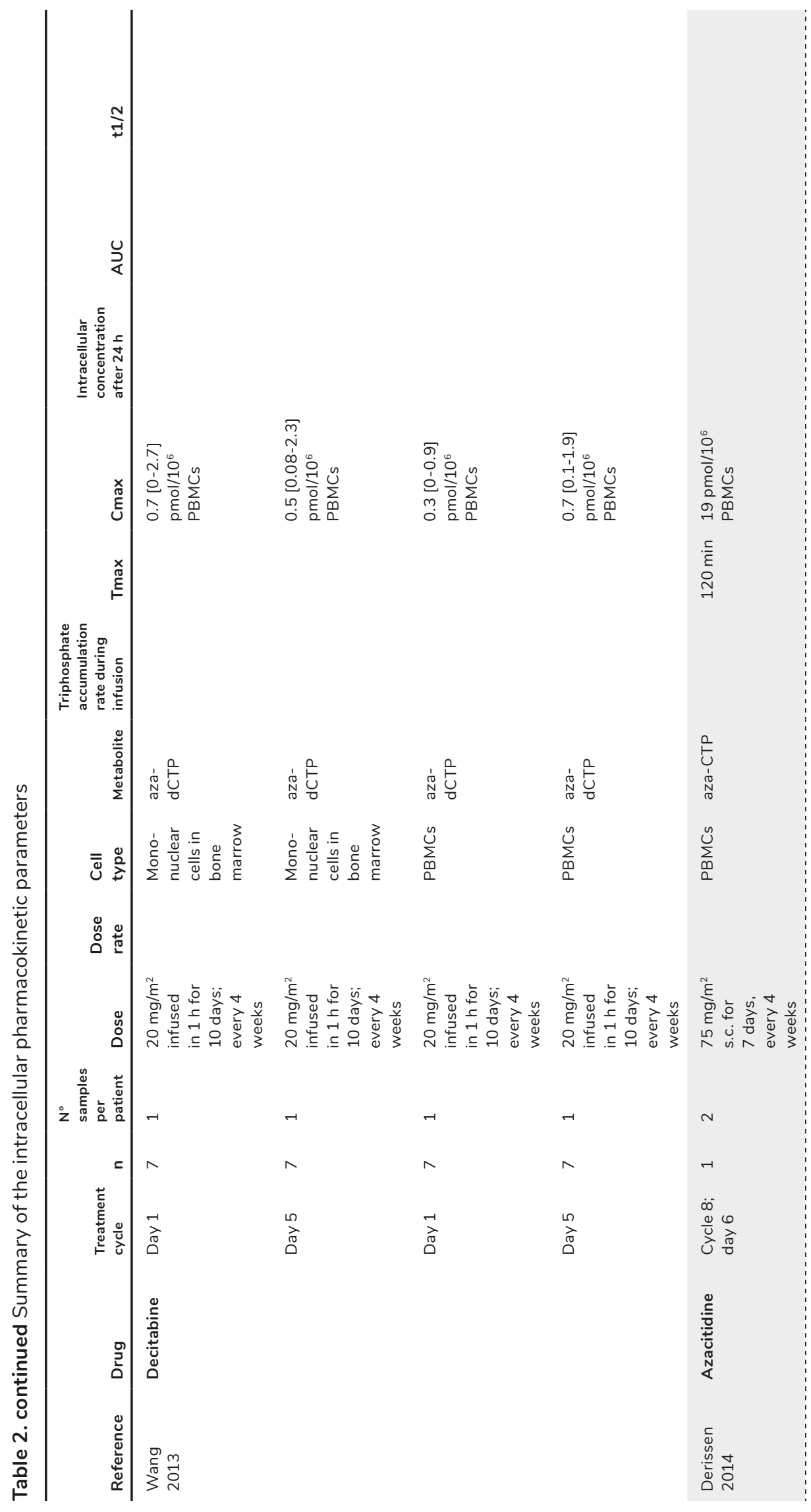




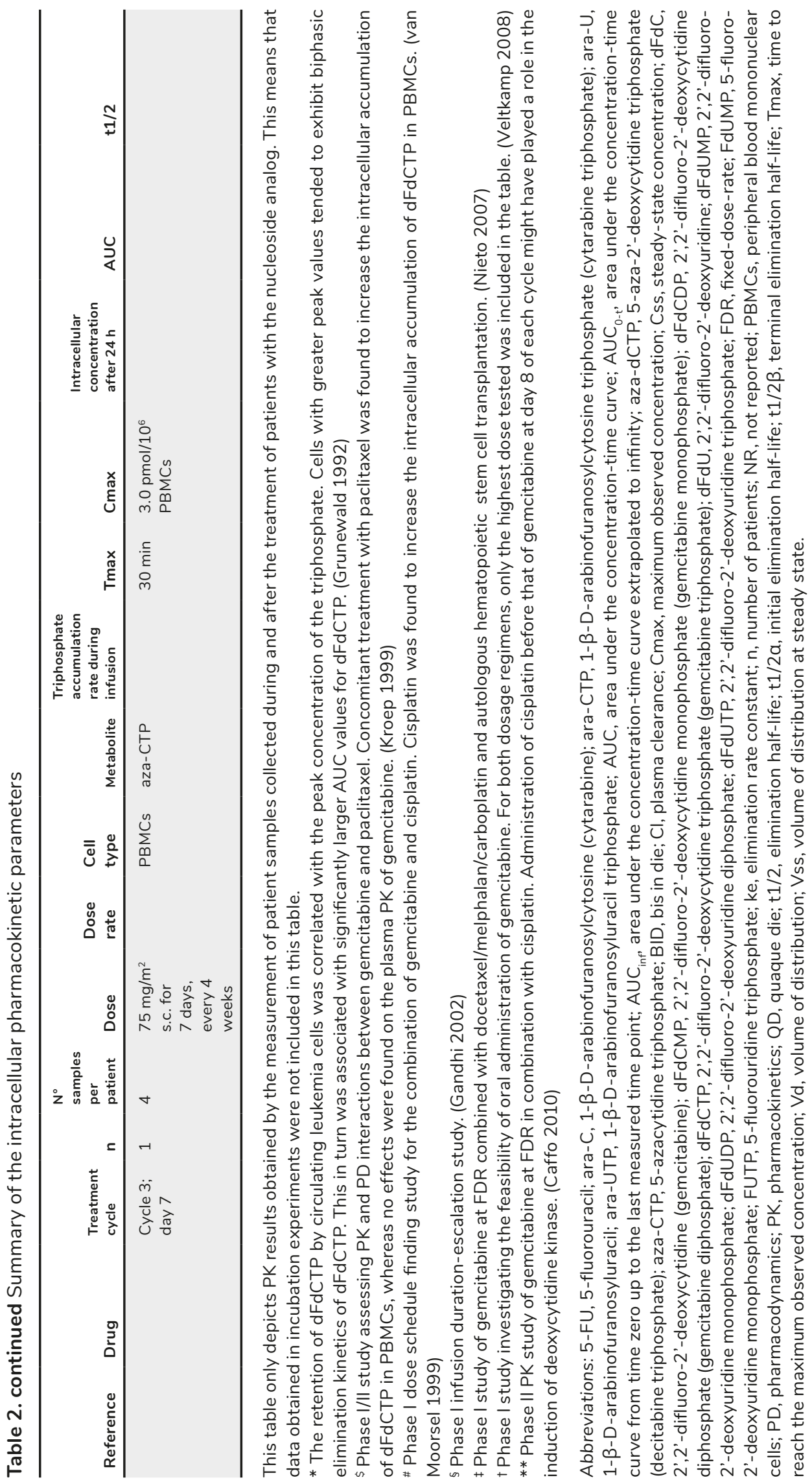




\section{REFERENCES}

[1] de Vos D, van Overveld W. Decitabine: a historical review of the development of an epigenetic drug. Ann Hemato/ 2005;84 Suppl 1:3-8.

[2] McCredie KB, Bodey GP, Burgess MA, et al. Treatment of acute leukemia with 5-azacytidine (NSC-102816). Cancer Chemother reports 1973;57:319-23.

[3] Vogler WR, Miller DS, Keller JW. 5-Azacytidine (NSC 102816): a new drug for the treatment of myeloblastic leukemia. Blood 1976;48:3317.

[4] Abele R, Clavel M, Dodion P, et al. The EORTC Early Clinical Trials Cooperative Group experience with 5-aza-2'-deoxycytidine (NSC 127716) in patients with colo-rectal, head and neck, renal carcinomas and malignant melanomas. Eur J Cancer Clin Oncol 1987;23:1921-4.

[5] Merriman RL, Hertel LW, Schultz RM, et al. Comparison of the antitumor activity of gemcitabine and ara- $\mathrm{C}$ in a panel of human breast, colon, lung and pancreatic xenograft models. Invest New Drugs 1996;14:243-7.

[6] Hoff PM, Cassidy J, Schmoll HJ. The evolution of fluoropyrimidine therapy: from intravenous to oral. Oncologist 2001;6 Suppl 4:3-11.

[7] Wijermans PW, Krulder JW, Huijgens PC, et al. Continuous infusion of low-dose 5-Aza-2'deoxycytidine in elderly patients with highrisk myelodysplastic syndrome. Leukemia 1997;11 Suppl 1:S19-23.

[8] Wijermans P, Lübbert M, Verhoef G, et al. Low-dose 5-aza-2'-deoxycytidine, a DNA hypomethylating agent, for the treatment of high-risk myelodysplastic syndrome: a multicenter phase II study in elderly patients. J Clin Oncol 2000;18:956-62.

[9] Silverman LR, Holland JF, Weinberg RS, et al. Effects of treatment with 5-azacytidine on the in vivo and in vitro hematopoiesis in patients with myelodysplastic syndromes. Leukemia 1993;7 Suppl 1:21-9.

[10] Silverman LR, Demakos EP, Peterson BL, et al. Randomized controlled trial of azacitidine in patients with the myelodysplastic syndrome: a study of the cancer and leukemia group B. J Clin Oncol 2002;20:2429-40.
[11] Derissen EJB, Beijnen JH, Schellens JHM. Concise drug review: azacitidine and decitabine. Oncologist 2013;18:619-24.

[12] Rutman RJ, Cantarow A, Paschkis KE. Studies in 2-acetylaminofluorene carcinogenesis. III. The utilization of uracil-2-C14 by preneoplastic rat liver and rat hepatoma. Cancer Res 1954;14:119-23.

[13] Grem JL. 5-Fluorouracil: forty-plus and still ticking. A review of its preclinical and clinical development. Invest New Drugs 2000;18:299-313.

[14] Kufe D, Pollock R, Weichselbaum R, et al. Pyrimidine analogs. In: Cancer Medicine. 2003.

[15] Reigner B, Blesch K, Weidekamm E. Clinical pharmacokinetics of capecitabine. Clin Pharmacokinet 2001;40:85-104.

[16] Diasio RB, Harris BE. Clinical pharmacology of 5-fluorouracil. Clin Pharmacokinet 1989;16:215-37.

[17] Chaudhuri NK, Mukherjee KL, Heidelberger C. Studies on fluorinated pyrimidines: VII - The degradative pathway. Biochem Pharmacol 1959;1:328-341.

[18] Ardalan B, Glazer R. An update on the biochemistry of 5-fluorouracil. Cancer Treat Rev 1981;8:157-67.

[19] Saif MW, Choma A, Salamone SJ, et al. Pharmacokinetically guided dose adjustment of 5-fluorouracil: A rational approach to improving therapeutic outcomes. J Natl Cancer Inst 2009;101:1543-1552.

[20] Etienne MC, Lagrange JL, Dassonville O, et al. Population study of dihydropyrimidine dehydrogenase in cancer patients. J Clin Oncol 1994;12:2248-53.

[21] Lu Z, Zhang R, Diasio RB. Dihydropyrimidine dehydrogenase activity in human peripheral blood mononuclear cells and liver: population characteristics, newly identified deficient patients, and clinical implication in 5-fluorouracil chemotherapy. Cancer Res 1993;53:5433-8.

[22] Mattison LK, Fourie J, Desmond RA, et al. Increased prevalence of dihydropyrimidine dehydrogenase deficiency in AfricanAmericans compared with Caucasians. Clin Cancer Res 2006;12:5491-5495. 
[23] De Bono JS, Twelves CJ. The oral fluorinated pyrimidines. Invest New Drugs 2001;19:4159.

[24] Álvarez P, Marchal JA, Boulaiz $\mathrm{H}$, et al. 5-Fluorouracil derivatives: a patent review. Expert Opin Ther Pat 2012;22:107-23.

[25] Piedbois P. Efficacy of intravenous continuous infusion of fluorouracil compared with bolus administration in advanced colorectal cancer. J Clin Oncol 1998;16:301-308.

[26] Gamelin EC, Danquechin-Dorval EM, Dumesnil YF, et al. Relationship between 5-fluorouracil (5-FU) dose intensity and therapeutic response in patients with advanced colorectal cancer receiving infusional therapy containing 5-FU. Cancer 1996;77:441-51.

[27] Miwa M, Ura M, Nishida M, et al. Design of a novel oral fluoropyrimidine carbamate, capecitabine, which generates 5 -fluorouracil selectively in tumours by enzymes concentrated in human liver and cancer tissue. Eur J Cancer 1998;34:1274-81.

[28] Ishikawa T, Utoh M, Sawada N, et al. Tumor selective delivery of 5 -fluorouracil by capecitabine, a new oral fluoropyrimidine carbamate, in human cancer xenografts. Biochem Pharmacol 1998;55:1091-7.

[29] Schüller J, Cassidy J, Dumont E, et al. Preferential activation of capecitabine in tumor following oral administration to colorectal cancer patients. Cancer Chemother Pharmacol 2000;45:291-297.

[30] Budman DR, Meropol NJ, Reigner B, et al. Preliminary studies of a novel oral fluoropyrimidine carbamate: capecitabine. J Clin Oncol 1998;16:1795-802.

[31] Wohlhueter RM, Mclvor RS, Plagemann PG. Facilitated transport of uracil and 5-fluorouracil, and permeation of orotic acid into cultured mammalian cells. I Cell Physiol 1980;104:309-19.

[32] Longley DB, Harkin DP, Johnston PG. 5-Fluorouracil: Mechanisms of Action and Clinical Strategies. Nat Rev Cancer 2003;3:330-8.

[33] Nies AT, Magdy T, Schwab M, et al. Role of $A B C$ transporters in fluoropyrimidine-based chemotherapy response. Adv Cancer Res 2015;125:217-43.

[34] Grem J. Mechanisms of Action and Modulation of Fluorouracil. Semin Radiat Oncol 1997;7:249-259.
[35] Wilson PM, Danenberg P V., Johnston PG, et al. Standing the test of time: Targeting thymidylate biosynthesis in cancer therapy. Nat Rev Clin Oncol 2014;11:282-298.

[36] Peters GJ, Laurensse E, Leyva A, et al. Sensitivity of human, murine, and rat cells to 5-fluorouracil and 5'-deoxy-5-fluorouridine in relation to drug-metabolizing enzymes. Cancer Res 1986;46:20-8.

[37] Ciccolini J, Peillard L, Evrard a, et al. Enhanced antitumor activity of 5-fluorouracil in combination with 2'-deoxyinosine in human colorectal cell lines and human colon tumor xenografts. Clin Cancer Res 2000;6:15291535.

[38] Ciccolini J, Peillard L, Aubert C, et al. Monitoring of the intracellular activation of 5 -fluorouracil to deoxyribonucleotides in HT29 human colon cell line: application to modulation of metabolism and cytotoxicity study. Fundam Clin Pharmacol 2000;14:147154.

[39] Kamm YJL, Peters GJ, Hull WE, et al. Correlation between 5-fluorouracil metabolism and treatment response in two variants of $\mathrm{C} 26$ murine colon carcinoma. $\mathrm{Br} J$ Cancer 2003;89:754-62.

[40] Spears CP, Gustavsson BG, Mitchell MS, et al. Thymidylate synthetase inhibition in malignant tumors and normal liver of patients given intravenous 5-fluorouracil. Cancer Res 1984;44:4144-50.

[41] Spears CP, Gustavsson BG, Berne M, et al. Mechanisms of innate resistance to thymidylate synthase inhibition after 5-fluorouracil. Cancer Res 1988;48:58945900.

[42] Peters GJ, Lankelma J, Kok RM, et al. Prolonged retention of high concentrations of 5 -fluorouracil in human and murine tumors as compared with plasma. Cancer Chemother Pharmacol 1993;31:269-76.

[43] Moran RG, Spears CP, Heidelberger C. Biochemical determinants of tumor sensitivity to 5-fluorouracil: ultrasensitive methods for the determination of 5-fluoro2'-deoxyuridylate, 2'-deoxyuridylate, and thymidylate synthetase. Proc Natl Acad SciU S A 1979;76:1456-60.

[44] Derissen EJB, Hillebrand MJX, Rosing H, et al. Development of an LC-MS/MS assay for the quantitative determination of the intracellular 5 -fluorouracil nucleotides responsible for the anticancer effect of 5-fluorouracil. J Pharm Biomed Anal 2015;110:58-66. 
[45] Derissen EJB, Jacobs BAW, Huitema ADR, et al. Exploring the intracellular pharmacokinetics of the 5-fluorouracil nucleotides during capecitabine treatment. Br J Clin Pharmacol 2016;81:949-57.

[46] Maring JG, Wachters FM, Slijfer M, et al. Pharmacokinetics of gemcitabine in nonsmall-cell lung cancer patients: Impact of the 79A $>$ C cytidine deaminase polymorphism. Eur J Clin Pharmacol 2010;66:611-617.

[47] DeAngelis LM, Kreis W, Chan K, et al. Pharmacokinetics of ara-C and ara- $U$ in plasma and CSF after high-dose administration of cytosine arabinoside. Cancer Chemother Pharmacol 1992;29:173-177.

[48] Hamada A, Kawaguchi T, Nakano M. Clinical pharmacokinetics of cytarabine formulations. Clin Pharmacokinet 2002;41:705-718.

[49] Burk M, Heyll A, Arning $M$, et al. Pharmacokinetics of high-dose cytarabine and its deamination product--a reappraisal. Leuk Lymphoma 1997;27:321-7.

[50] Gandhi V, Xu YZ, Estey E. Accumulation of arabinosyluracil 5'-triphosphate during arabinosylcytosine therapy in circulating blasts of patients with acute myelogenous leukemia. Clin Cancer Res 1998;4:1719-26.

[51] Mackey JR, Mani RS, Selner M, et al. Functional nucleoside transporters are required for gemcitabine influx and manifestation of toxicity in cancer cell lines. Cancer Res 1998;58:4349-57.

[52] Ritzel MW, Ng AM, Yao SY, et al. Recent molecular advances in studies of the concentrative $\mathrm{Na}+$-dependent nucleoside transporter (CNT) family: identification and characterization of novel human and mouse proteins ( $\mathrm{hCNT3}$ and $\mathrm{mCNT3}$ ) broadly selective for purine and pyrimidine nucleosides. Mol Membr Biol 2001;18:65-72.

[53] Damaraju VL, Mowles D, Yao S, et al. Role of human nucleoside transporters in the uptake and cytotoxicity of azacitidine and decitabine. Nucleosides, Nucleotides and Nucleic Acids 2012;31:236-255.

[54] Clarke ML, Mackey JR, Baldwin SA, et al. The role of membrane transporters in cellular resistance to anticancer nucleoside drugs. Cancer Treat Res 2002;112:27-47.

[55] Kufe DW, Major PP, Egan EM, et al. Correlation of cytotoxicity with incorporation of ara-C into DNA. J Biol Chem 1980;255:8997-9000.
[56] Major PP, Egan EM, Beardsley GP, et al. Lethality of human myeloblasts correlates with the incorporation of arabinofuranosylcytosine into DNA. Proc Natl Acad Sci U S A 1981;78:3235-9.

[57] Kufe D, Spriggs D, Egan EM, et al. Relationships among Ara-CTP pools, formation of (Ara-C) DNA, and cytotoxicity of human leukemic cells. Blood 1984;64:54-8.

[58] Chou TC, Arlin Z, Clarkson BD, et al. Metabolism of 1-beta-Darabinofuranosylcytosine in human leukemic cells. Cancer Res 1977;37:3561-70.

[59] Liliemark JO, Plunkett W, Dixon DO. Relationship of 1-beta-Darabinofuranosylcytosine in plasma to 1-beta-D-arabinofuranosylcytosine 5 '-triphosphate levels in leukemic cells during treatment with high-dose 1-betaD-arabinofuranosylcytosine. Cancer Res 1985;45:5952-7.

[60] Plunkett W, Liliemark JO, Adams TM, et al. Saturation of 1-beta-Darabinofuranosylcytosine 5'-triphosphate accumulation in leukemia cells during highdose 1-beta-D-arabinofuranosylcytosine therapy. Cancer Res 1987;47:3005-11.

[61] Plunkett W, Liliemark JO, Estey E, et al. Saturation of ara-CTP accumulation during high-dose ara-C therapy: pharmacologic rationale for intermediate-dose ara-C. Semin Oncol 1987;14:159-66.

[62] Hiddemann W, Schleyer E, Unterhalt $M$, et al. Differences in the intracellular pharmacokinetics of cytosine arabinoside (AraC) between circulating leukemic blasts and normal mononuclear blood cells. Leukemia 1992;6:1273-80.

[63] Kantarjian HM, Estey EH, Plunkett W, et al. Phase I-II clinical and pharmacologic studies of high-dose cytosine arabinoside in refractory leukemia. Am J Med 1986;81:387394.

[64] Harris AL, Grahame-Smith DG. Variation in sensitivity of DNA synthesis to ara-C in acute myeloid leukaemia. $\mathrm{Br} J$ Haematol 1980;45:371-9.

[65] Köhl U, Schwabe D, Montag E, et al. Formation of cytosine arabinoside-5'-triphosphate in different cultured lymphoblastic leukaemic cells with reference to their drug sensitivity. Eur J Cancer 1995;31A:209-14. 
[66] Löwenberg B, Downing JR, Burnett A. Acute myeloid leukemia. N Engl J Med 1999;341:1051-62.

[67] Breithaupt $H$, Pralle H, Eckhardt T, et al. Clinical results and pharmacokinetics of high-dose cytosine arabinoside (HD ARA-C). Cancer 1982;50:1248-57.

[68] Early AP, Preisler HD, Slocum H, et al. A pilot study of high-dose 1-beta-Darabinofuranosylcytosine for acute leukemia and refractory lymphoma: clinical response and pharmacology. Cancer Res 1982;42:1587-94.

[69] Capizzi RL, Yong JL, Cheng E. Alteration of the pharmacokinetics of high-dose Ara- $\mathrm{C}$ by its metabolite, high Ara- $U$ in patients with acute leukemia. J Clin Oncol 1983;1:763-771.

[70] Herzig RH, Lazarus HM, Wolff SN, et al. Highdose cytosine arabinoside therapy with and without anthracycline antibiotics for remission reinduction of acute nonlymphoblastic leukemia. J Clin Oncol 1985;3:992-7.

[71] Wolff SN, Marion J, Stein RS, et al. Highdose cytosine arabinoside and daunorubicin as consolidation therapy for acute nonlymphocytic leukemia in first remission: a pilot study. Blood 1985;65:1407-11.

[72] Lazarus HM, Herzig RH, Herzig GP, et al. Central nervous system toxicity of highdose systemic cytosine arabinoside. Cancer 1981;48:2577-82.

[73] Salinsky MC, Levine RL, Aubuchon JP, et al. Acute cerebellar dysfunction with high-dose ARA-C therapy. Cancer 1983;51:426-9.

[74] Andersson BS, Cogan BM, Keating MJ, et al. Subacute pulmonary failure complicating therapy with high-dose Ara-C in acute leukemia. Cancer 1985;56:2181-4.

[75] Hwang TL, Yung WK, Estey EH, et al. Central nervous system toxicity with high-dose Ara-C. Neurology 1985;35:1475-9.

[76] Nand S, Messmore HL, Patel R, et al. Neurotoxicity associated with systemic high-dose cytosine arabinoside. J Clin Oncol 1986;4:571-5.

[77] Lopez JA, Agarwal RP. Acute cerebellar toxicity after high-dose cytarabine associated with CNS accumulation of its metabolite, uracil arabinoside. Cancer Treat Rep 1984;68:130910.
[78] Mantovani L, Hasenclever D, Krahl R, et al. Intermediate-dose Cytarabine Treatment Delivered at Moderate Infusion Rates for De Novo Acute Myeloid Leukemia-Results of a phase 1-II Study. Leuk Lymphoma 2002;43:265-274.

[79] Mayer RJ, Davis RB, Schiffer CA, et al. Intensive postremission chemotherapy in adults with acute myeloid leukemia. Cancer and Leukemia Group B. N Engl J Med 1994;331:896-903.

[80] Bishop JF, Matthews JP, Young GA, et al. A randomized study of high-dose cytarabine in induction in acute myeloid leukemia. Blood 1996;87:1710-7.

[81] Weick JK, Kopecky KJ, Appelbaum FR, et al. A randomized investigation of high-dose versus standard-dose cytosine arabinoside with daunorubicin in patients with previously untreated acute myeloid leukemia: a Southwest Oncology Group study. Blood 1996;88:2841-2851.

[82] Farag SS, Ruppert AS, Mrózek K, et al. Outcome of induction and postremission therapy in younger adults with acute myeloid leukemia with normal karyotype: a cancer and leukemia group B study. J Clin Oncol 2005;23:482-93.

[83] Büchner T, Berdel WE, Schoch C, et al. Double induction containing either two courses or one course of high-dose cytarabine plus mitoxantrone and postremission therapy by either autologous stem-cell transplantation or by prolonged maintenance for acute myeloid leukemia. J Clin Oncol 2006;24:2480-2489.

[84] Döhner H, Estey EHE, Amadori S, et al. Diagnosis and management of acute myeloid leukemia in adults: recommendations from an international expert panel, on behalf of the European LeukemiaNet. Blood 2010;115:453474.

[85] Löwenberg B. Sense and nonsense of highdose cytarabine for acute myeloid leukemia. Blood 2013;121:26-8.

[86] Löwenberg B, Pabst T, Vellenga E, et al. Cytarabine dose for acute myeloid leukemia. N Engl J Med 2011;364:1027-1036.

[87] Estey E, Plunkett W, Dixon D, et al. Variables predicting response to high dose cytosine arabinoside therapy in patients with refractory acute leukemia. Leukemia 1987;1:580-3. 
[88] Estey EH, Keating MJ, McCredie KB, et al. Cellular ara-CTP pharmacokinetics, response, and karyotype in newly diagnosed acute myelogenous leukemia. Leukemia 1990;4:959.

[89] Rustum YM, Preisler HD. Correlation between leukemic cell retention of 1-beta-Darabinofuranosylcytosine 5'-triphosphate and response to therapy. Cancer Res 1979;39:429.

[90] Preisler HD, Rustum Y, Priore RL. Relationship between leukemic cell retention of cytosine arabinoside triphosphate and the duration of remission in patients with acute nonIymphocytic leukemia. Eur J Cancer Clin Oncol 1985;21:23-30.

[91] Preisler HD, Rustum YM, Azarnia N, et al. Abrogation of the prognostic significance of low leukemic cell retention of cytosine arabinoside triphosphate by intensification of therapy and by alteration in the dose and schedule of administration of cytosine arabinoside. Cancer Chemother Pharmacol 1987;19:69-74.

[92] Gandhi V, Huang P, Plunkett W. Metabolism and action of arabinosyluracil 5'-triphosphate in acute myelogenous leukemia blasts. Proc Am Assoc Cancer Res 1992;33:545.

[93] Gandhi V, Chapman A, Huang P, et al. Sequence specific inhibition of DNA polymerase a by 5'-triphosphates of 1- $\beta$-D-arabinofuranosylcytosine and arabinosyluracil. Proc Am Assoc Cancer Res 1993;34:349.

[94] Müller WE, Zahn RK. Metabolism of 1-beta-Darabinofuranosyluracil in mouse L5178Y cells. Cancer Res 1979;39:1102-7.

[95] Ono K, Ohashi A, Ogasawara M, et al. Inhibition of deoxyribonucleic acid polymerases from murine cells and oncornavirus by 5-alkylated derivatives of 1-beta-Darabinofuranosyluracil 5'-triphosphate: substituent effects on inhibitory action. Biochemistry 1981;20:5088-93.

[96] Yang JL, Cheng EH, Capizzi RL, et al. Effect of uracil arabinoside on metabolism and cytotoxicity of cytosine arabinoside in L5178Y murine leukemia. J Clin Invest 1985;75:141-6.

[97] Abbruzzese JL, Grunewald R, Weeks EA, et al. A phase I clinical, plasma, and cellular pharmacology study of gemcitabine. J Clin Oncol 1991;9:491-8.
[98] Wong A, Soo RA, Yong W-P, et al. Clinical pharmacology and pharmacogenetics of gemcitabine. Drug Metab Rev 2009;41:7788.

[99] Gandhi V, Legha J, Chen F, et al. Excision of 2',2'-difluorodeoxycytidine (gemcitabine) monophosphate residues from DNA. Cancer Res 1996;56:4453-4459.

[100] Huang P, Chubb S, Hertel L, et al. Action of 2', 2'-difluorodeoxycytidine on DNA synthesis. Cancer Res 1991;51:6110-6117.

[101] Heinemann V, Xu YZ, Chubb S, et al. Inhibition of ribonucleotide reduction in CCRF-CEM cells by 2',2'-difluorodeoxycytidine. Mol Pharmacol 1990;38:567-572.

[102] Heinemann V, Xu YZ, Chubb S, et al. Cellular elimination of 2',2'-difluorodeoxycytidine 5'-triphosphate: A mechanism of selfpotentiation. Cancer Res 1992;52:533-539.

[103] de Sousa Cavalcante L, Monteiro G. Gemcitabine: Metabolism and molecular mechanisms of action, sensitivity and chemoresistance in pancreatic cancer. Eur J Pharmacol 2014;741:8-16.

[104] Hunsucker SA, Mitchell BS, Spychala J. The 5 '-nucleotidases as regulators of nucleotide and drug metabolism. Pharmacol Ther 2005;107:1-30.

[105] Xu Y-Z, Plunkett W. Modulation of deoxycytidylate deaminase in intact human leukemia cells. Action of 2',2'-difluorodeoxycytidine. Biochem Pharmacol 1992;44:1819-1827.

[106] Mackey JR, Yao SY, Smith KM, et al. Gemcitabine transport in xenopus oocytes expressing recombinant plasma membrane mammalian nucleoside transporters. I NatI Cancer Inst 1999;91:1876-1881.

[107] Shewach DS, Reynolds KK, Hertel L. Nucleotide specificity of human deoxycytidine kinase. Mol Pharmacol 1992;42:518-24.

[108] Plunkett W, Huang P, Searcy CE, et al. Gemcitabine: preclinical pharmacology and mechanisms of action. Semin Oncol 1996;23:3-15.

[109] Bouffard DY, Laliberte J, Momparler RL. Kinetic studies on 2',2'-difluorodeoxycytidine (gemcitabine) with purified human deoxycytidine kinase and cytidine deaminase. Biochem Pharmacol 1993;45:1857-1861. 
[110] Grunewald R, Abbruzzese JL, Tarassoff P, et al. Saturation of 2',2'-difluorodeoxycytidine 5'-triphosphate accumulation by mononuclear cells during a phase I trial of gemcitabine. Cancer Chemother Pharmacol 1991;27:258262.

[111] Grunewald R, Kantarjian H, Du M, et al. Gemcitabine in leukemia: a phase I clinical, plasma, and cellular pharmacology study. J Clin Oncol 1992;10:406-13.

[112] Grunewald R, Kantarjian H, Keating $\mathrm{MJ}$, et al. Pharmacologically directed design of the dose rate and schedule of 2',2'-difluorodeoxycytidine (Gemcitabine) administration in leukemia. Cancer Res 1990;50:6823-6.

[113] Patel SR, Gandhi V, Jenkins J, et al. Phase II clinical investigation of gemcitabine in advanced soft tissue sarcomas and window evaluation of dose rate on gemcitabine triphosphate accumulation. J Clin Oncol 2001;19:3483-9.

[114] Gandhi V, Plunkett W, Du M, et al. Prolonged infusion of gemcitabine: Clinical and pharmacodynamic studies during a phase I trial in relapsed acute myelogenous leukemia. J Clin Oncol 2002;20:665-673.

[115] Cattel L, Airoldi M, Delprino L, et al. Pharmacokinetic evaluation of gemcitabine and 2', 2'-difluorodeoxycytidine-5'triphosphate after prolonged infusion in patients affected by different solid tumors. Ann Oncol 2006;17 Suppl 5:v142-7.

[116] Grimison P, Galettis P, Manners S, et al. Randomized crossover study evaluating the effect of gemcitabine infusion dose rate: evidence of auto-induction of gemcitabine accumulation. J Clin Oncol 2007;25:5704-9.

[117] Van Haperen VWTR, Veerman G, Boven E, et al. Schedule dependence of sensitivity to 2',2'-difluorodeoxycytidine (gemcitabine) in relation to accumulation and retention of its triphosphate in solid tumour cell lines and solid tumours. Biochem Pharmacol 1994;48:1327-1339.

[118] Tempero M, Plunkett W, Ruiz van Haperen VW, et al. Randomized Phase II Comparison of Dose-Intense Gemcitabine: Thirty-Minute Infusion and Fixed Dose Rate Infusion in Patients With Pancreatic Adenocarcinoma. J Clin Oncol 2003;21:3402-3408.
[119] Guan Z, Wang Y, Maoleekoonpairoj S, et al. Prospective randomised phase II study of gemcitabine at standard or fixed dose rate schedule in unresectable hepatocellular carcinoma. BrJ Cancer 2003;89:1865-1869.

[120] Ceribelli A, Gridelli C, De Marinis F, et al. Prolonged gemcitabine infusion in advanced non-small cell lung carcinoma: A randomized Phase II study of two different schedules in combination with cisplatin. Cancer 2003;98:337-343.

[121] Soo RA, Wang LZ, Tham LS, et al. A multicentre randomised phase II study of carboplatin in combination with gemcitabine at standard rate or fixed dose rate infusion in patients with advanced stage non-small-cell lung cancer. Ann Oncol 2006;17:1128-1133.

[122] Cappuzzo F, Novello S, De Marinis F, et al. A randomized phase II trial evaluating standard (50 $\mathrm{mg} / \mathrm{min}$ ) versus low $(10 \mathrm{mg} /$ $\min$ ) infusion duration of gemcitabine as first-line treatment in advanced non-small-cell lung cancer patients who are not eligible for platinum-based chemotherapy. Lung Cancer 2006;52:319-325.

[123] Gridelli C, Gallo C, Ceribelli A, et al. Factorial phase III randomised trial of rofecoxib and prolonged constant infusion of gemcitabine in advanced non-small-cell lung cancer: the GEmcitabine-COxib in NSCLC (GECO) study. Lancet Oncol 2007;8:500-512.

[124] Poplin E, Feng Y, Berlin J, et al. Phase III, Randomized Study of Gemcitabine and Oxaliplatin Versus Gemcitabine (fixed-dose rate infusion) Compared With Gemcitabine (30-minute infusion) in Patients With Pancreatic Carcinoma E6201: A Trial of the Eastern Cooperative Oncology Group. J Clin Oncol 2009;27:3778-3785.

[125] Fossella F V, Lippman SM, Shin DM, et al. Maximum-tolerated dose defined for singleagent gemcitabine: a phase I dose-escalation study in chemotherapy-naive patients with advanced non-small-cell lung cancer. J Clin Oncol 1997;15:310-6.

[126] Brand R, Capadano M, Tempero M. A phase I trial of weekly gemcitabine administered as a prolonged infusion in patients with pancreatic cancer and other solid tumors. Invest New Drugs 1997;15:331-341.

[127] Touroutoglou N, Gravel D, Raber MN, et al. Clinical results of a pharmacodynamicallybased strategy for higher dosing of gemcitabine in patients with solid tumors. Ann Oncol 1998;9:1003-1008. 
[128] Ruiz van Haperen VW, Veerman G, Eriksson S, et al. Development and molecular characterization of a 2',2'-difluorodeoxycytidine-resistant variant of the human ovarian carcinoma cell line A2780. Cancer Res 1994:54:4138-43.

[129] Veltkamp SA, Pluim D, van Eijndhoven MAJ, et al. New insights into the pharmacology and cytotoxicity of gemcitabine and 2',2'-difluorodeoxyuridine. Mol Cancer Ther 2008;7:2415-25.

[130] Honeywell RJ, Ruiz Van Haperen VWT, Veerman G, et al. Inhibition of thymidylate synthase by 2',2'-difluoro-2'-deoxycytidine (Gemcitabine) and its metabolite 2',2'-difluoro-2'-deoxyuridine. Int J Biochem Cell Biol 2015;60:73-81.

[131] Derissen EJB, Huitema ADR, Rosing $H$, et al. Intracellular pharmacokinetics of gemcitabine, its deaminated metabolite 2',2'-difluorodeoxyuridine and their nucleotides. $\mathrm{Br} J \mathrm{Clin}$ Pharmacol 2018;84:1279-1289.

[132] Veltkamp SA, Jansen RS, Callies S, et al. Oral administration of gemcitabine in patients with refractory tumors: a clinical and pharmacologic study. Clin Cancer Res 2008;14:3477-86.

[133] van Moorsel CJ, Kroep JR, Pinedo HM, et al. Pharmacokinetic schedule finding study of the combination of gemcitabine and cisplatin in patients with solid tumors. Ann Oncol 1999:10:441-448.

[134] Kroep JR, Giaccone G, Voorn DA, et al. Gemcitabine and paclitaxel: pharmacokinetic and pharmacodynamic interactions in patients with non-small-cell lung cancer. J Clin Oncol 1999;17:2190-7.

[135] Veltkamp SA, Beijnen JH, Schellens JHM. Prolonged versus standard gemcitabine infusion: translation of molecular pharmacology to new treatment strategy. Oncologist 2008;13:261-76.

[136] Shord SS, Faucette SR, Gillenwater $\mathrm{HH}$, et al. Gemcitabine pharmacokinetics and interaction with paclitaxel in patients with advanced non-small-cell lung cancer. Cancer Chemother Pharmacol 2003;51:328-336.

[137] Marcucci G, Silverman L, Eller M, et al. Bioavailability of azacitidine subcutaneous versus intravenous in patients with the myelodysplastic syndromes. J Clin Pharmacol 2005;45:597-602.
[138] Laille E, Goel S, Mita AC, et al. A phase I study in patients with solid or hematologic malignancies of the dose proportionality of subcutaneous azacitidine and its pharmacokinetics in patients with severe renal impairment. Pharmacotherapy 2014;34:440451.

[139] Chabner BA, Drake JC, Johns DG. Deamination of 5-azacytidine by a human leukemia cell cytidine deaminase. Biochem Pharmacol 1973;22:2763-5.

[140] Chabot GG, Bouchard J, Momparler RL. Kinetics of deamination of 5-aza-2'deoxycytidine and cytosine arabinoside by human liver cytidine deaminase and its inhibition by 3 -deazauridine, thymidine or uracil arabinoside. Biochem Pharmacol 1983;32:1327-1328.

[141] Cashen AF, Shah AK, Todt L, et al. Pharmacokinetics of decitabine administered as a 3-h infusion to patients with acute myeloid leukemia (AML) or myelodysplastic syndrome (MDS). Cancer Chemother Pharmacol 2008;61:759-766.

[142] Momparler RL. Pharmacology of 5-Aza-2'deoxycytidine (decitabine). Semin Hematol 2005;42:S9-16.

[143] Li LH, Olin EJ, Buskirk HH, et al. Cytotoxicity and mode of action of 5-azacytidine on L1210 leukemia. Cancer Res 1970;30:2760-9.

[144] Aimiuwu J, Wang $\mathrm{H}$, Chen $\mathrm{P}$, et al. RNAdependent inhibition of ribonucleotide reductase is a major pathway for 5 -azacytidine activity in acute myeloid leukemia. Blood 2012;119:5229-5238.

[145] Kaminskas E, Farrell AT, Wang Y-C, et al. FDA drug approval summary: azacitidine (5-azacytidine, Vidaza) for injectable suspension. Oncologist 2005;10:176-82.

[146] Momparler RL. Molecular, cellular and animal pharmacology of 5-aza-2'-deoxycytidine. Pharmacol Ther 1985;30:287-99.

[147] Uchida T, Kinoshita T, Nagai H, et al. Hypermethylation of the p15INK4B gene in myelodysplastic syndromes. Blood 1997:90:1403-9.

[148] Christiansen DH, Anderson MK, PedersenBjergaard J. Methylation of p15 INK4B is common, is associated with deletion of genes on chromosome arm 7q and predicts a poor prognosis in therapy-related myelodysplasia and acute myeloid leukemia. Leukemia 2003;17:1813-1819. 
[149] Issa J-P. Epigenetic changes in the myelodysplastic syndrome. Hematol Oncol Clin North Am 2010;24:317-30.

[150] McCabe MT, Brandes JC, Vertino PM. Cancer DNA methylation: Molecular mechanisms and clinical implications. Clin Cancer Res 2009;15:3927-3937.

[151] Christman JK. 5-Azacytidine and 5-aza2'-deoxycytidine as inhibitors of DNA methylation: Mechanistic studies and their implications for cancer therapy. Oncogene 2002;21:5483-5495.

[152] EPAR Dacogen. European Medicines Agency. EMA/620205/2012, http://www.ema.europa. eu/docs/en_GB/document_library/EPAR_-_ Public_assessment_report/human/002221/ WC500133571.pdf.

[153] Kantarjian HM, Issa J-PJ. Decitabine dosing schedules. Semin Hematol 2005;42:S17-22.

[154] Oki Y, Aoki E, Issa JPJ. Decitabine-Bedside to bench. Crit Rev Oncol Hematol 2007;61:140152 .

[155] Jabbour E, Issa JP, Garcia-Manero G, et al. Evolution of decitabine development: Accomplishments, ongoing investigations, and future strategies. Cancer 2008;112:23412351.

[156] O'Dwyer K, Maslak P. Azacitidine and the beginnings of therapeutic epigenetic modulation. Expert Opin Pharmacother 2008;9:1981-1986.
[157] Fenaux P, Mufti GJ, Hellstrom-Lindberg E, et al. Efficacy of azacitidine compared with that of conventional care regimens in the treatment of higher-risk myelodysplastic syndromes: a randomised, open-label, phase III study. Lancet Oncol 2009;10:223-232.

[158] Kantarjian HM, Thomas XG, Dmoszynska A, et al. Multicenter, randomized, open-label, phase III trial of decitabine versus patient choice, with physician advice, of either supportive care or low-dose cytarabine for the treatment of older patients with newly diagnosed acute myeloid leukemia. J Clin Onco/ 2012;30:26707.

[159] Jansen RS, Rosing H, Wijermans PW, et al. Decitabine triphosphate levels in peripheral blood mononuclear cells from patients receiving prolonged low-dose decitabine administration: A pilot study. Cancer Chemother Pharmacol 2012;69:1457-1466.

[160] Wang $H$, Chen $P$, Wang J, et al. In vivo quantification of active decitabinetriphosphate metabolite: a novel pharmacoanalytical endpoint for optimization of hypomethylating therapy in acute myeloid leukemia. AAPS J 2013;15:242-9.

[161] Derissen EJB, Hillebrand MJX, Rosing H, et al. Quantitative determination of azacitidine triphosphate in peripheral blood mononuclear cells using liquid chromatography coupled with high-resolution mass spectrometry. J Pharm Biomed Anal 2014;90:7-14.

[162] Unnikrishnan A, Vo ANQ, Pickford R, et al. AZA-MS: A novel multiparameter mass spectrometry method to determine the intracellular dynamics of azacitidine therapy in vivo. Leukemia 2018;32:900-910. 


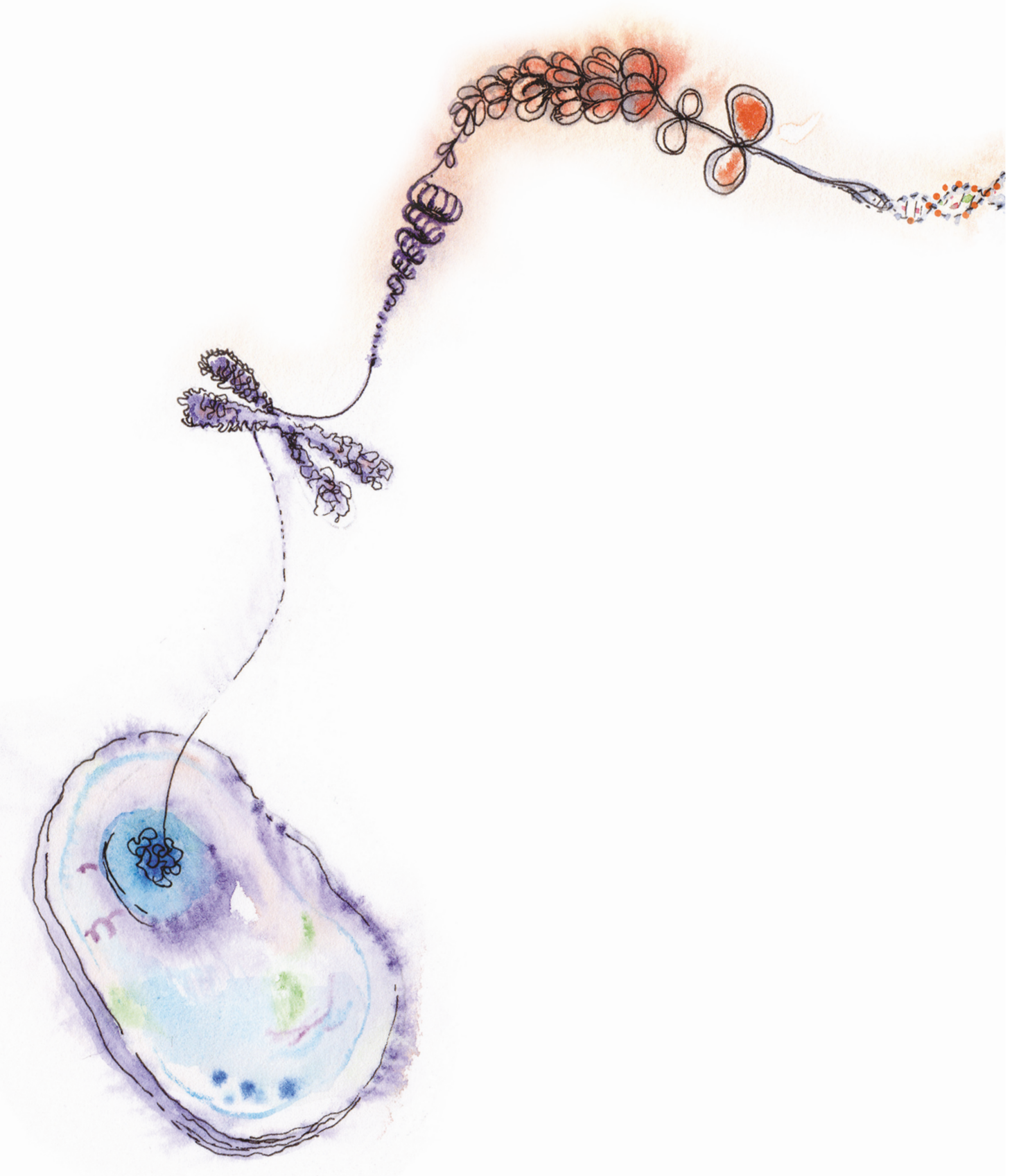




\section{Azacitidine and Decitabine}

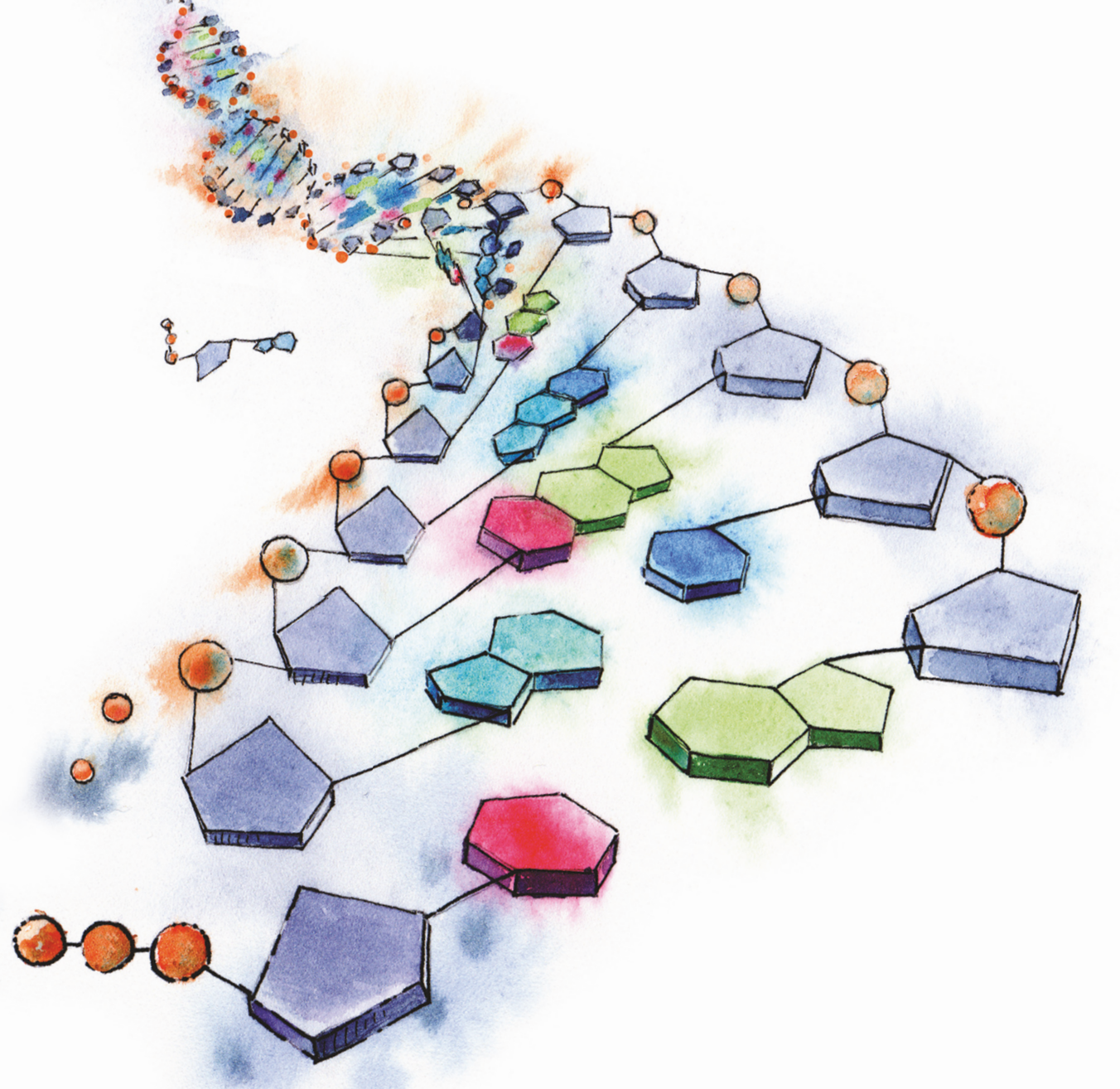




\section{CHAPTER 2.1}

Concise drug review: azacitidine and decitabine

The Oncologist 2013;18:619-624.

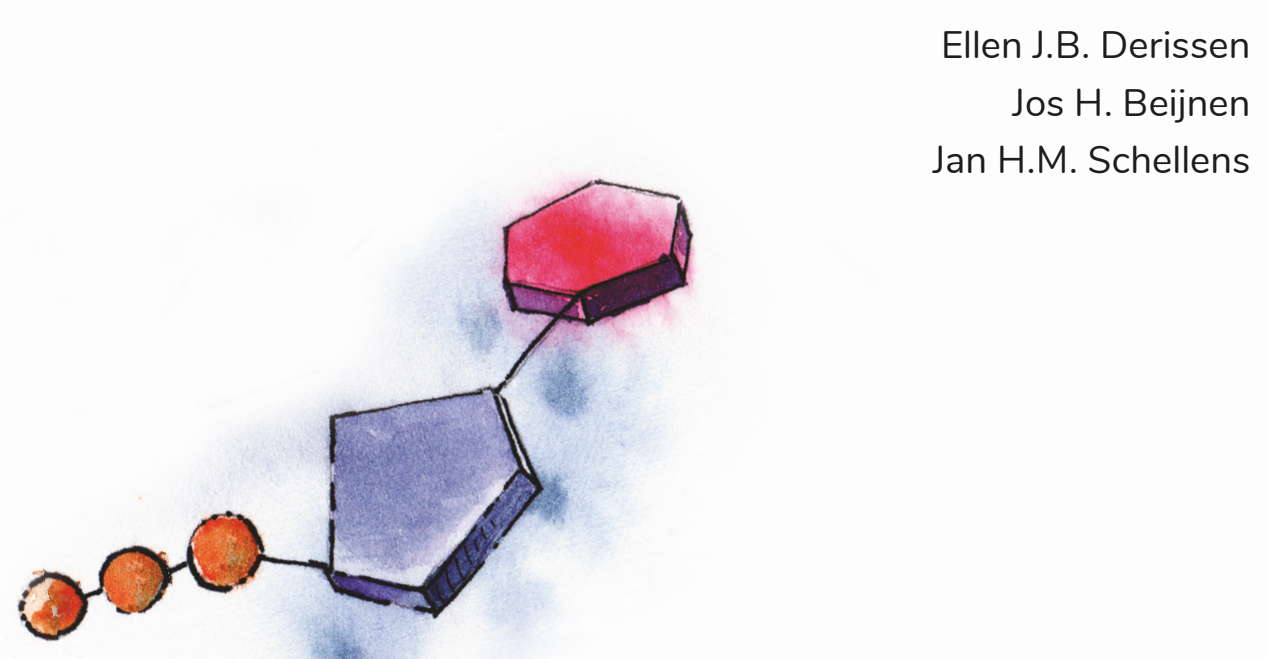




\section{IMPLICATIONS FOR PRACTICE}

The introduction of the hypomethylating agents azacitidine and decitabine has been a major advancement in the treatment of patients with higher-risk myelodysplastic syndromes, chronic myelomonocytic leukemia, and acute myeloid leukemia who are ineligible for more intensive treatments, such as allogeneic stem cell transplantation and induction chemotherapy. However, a number of uncertainties remain. Because only about $50 \%$ of patients respond to therapy with hypomethylating agents, identification of response-predicting biomarkers is warranted. In addition, the majority of responders relapse within two years; thus, identification of mechanisms of resistance is pivotal for further treatment optimization. This concise drug review summarizes the current state of treatment with the hypomethylating agents azacitidine and decitabine.

Keywords: azacitidine, decitabine, drug profile, DNA methyltransferase inhibitor, hypomethylating drug 


\section{INTRODUCTION}

The hypomethylating agents azacitidine (Vidaza $\left.{ }^{\circledR}\right)$ and decitabine (5-aza-2'deoxycytidine; Dacogen ${ }^{\circledR}$ ) are currently approved for the treatment of several specific forms of myelodysplastic syndromes (MDS), chronic myelomonocytic leukemia (CMML), and acute myeloid leukemia (AML), as depicted in Table 1.

The only potentially curative therapy for patients with higher-risk MDS (International Prognostic Scoring System intermediate-2 risk and high risk) is an allogeneic stem cell transplantation (allo-SCT). However, for the majority of patients, allo-SCT is not an option because of advanced age and/or comorbidities. In addition, the results obtained with intensive chemotherapy (when feasible) are often disappointing. For patients with MDS, the probability of complete remission after intensive chemotherapy is generally lower and the remission duration shorter than for patients with primary AML [1]. Therefore, the introduction of the hypomethylating agents has been a major advancement in the treatment of patients with higher-risk MDS who are ineligible for alloSCT. Table 2 summarizes the key pharmacologic features of these two agents.

The pivotal phase III trial investigating azacitidine treatment in patients with higher-risk MDS demonstrated a significant improvement of overall survival compared to supportive care or low-dose cytarabine. However, when compared to intensive chemotherapy, there was no significant difference in overall survival [2]. In the absence of undisputed, comparative data, it remains uncertain whether hypomethylating agents should always be preferred over intensive chemotherapy [3]. The main results of the phase III trials with azacitidine and decitabine are summarized in the Supplementary table at the end of this chapter.

In contrast to azacitidine, decitabine showed no beneficial effect on overall survival or time to $A M L$ in patients with MDS, even though the response percentages obtained with azacitidine and decitabine were almost the same $[2,4,5]$. The fact that a survival benefit in patients with MDS was found for azacitidine and not for decitabine does not necessarily indicate a discrepant pharmacology. The difference may also be explained by the differences in study 
Table 1. Summary of approved indications

\begin{tabular}{|c|c|c|}
\hline & EMA registration & FDA registration \\
\hline Azacitidine & $\begin{array}{l}\text { Treatment of adult patients, not } \\
\text { eligible for hematopoietic stem cell } \\
\text { transplantation, with: } \\
\text { - intermediate- } 2 \text { and high-risk } \\
\text { MDS, } \\
\text { - } \text { CMML with } 10-29 \% \\
\text { marrow blasts and without } \\
\text { myeloproliferative disorder, } \\
\text { - AML with } 20-30 \% \text { blasts and } \\
\text { multi-lineage dysplasia. }\end{array}$ & $\begin{array}{l}\text { Treatment of MDS according to } \\
\text { the FAB classification. }\end{array}$ \\
\hline \multirow[t]{2}{*}{ Decitabine } & $\begin{array}{l}\text { Not approved for the treatment of } \\
\text { MDS. }\end{array}$ & $\begin{array}{l}\text { Treatment of patients with } \\
\text { MDS of all FAB subtypes, and } \\
\text { intermediate-1, intermediate-2, } \\
\text { and high-risk IPSS groups. }\end{array}$ \\
\hline & $\begin{array}{l}\text { Treatment of older patients ( } \geq 65 \\
\text { years) with } A M L \text { who are not eligible } \\
\text { for treatment with standard induction } \\
\text { chemotherapy. }\end{array}$ & $\begin{array}{l}\text { Not approved for treatment of } \\
\text { elderly patients with AML. }\end{array}$ \\
\hline
\end{tabular}

Abbreviations: AML, acute myeloid leukemia; CMML, chronic myelomonocytic leukemia; EMA, European Medicines Agency; FAB, French-American-British; FDA, U.S. Food and Drug Administration; IPSS, International Prognostic Scoring System; MDS, myelodysplastic syndrome.

design, in particular the inclusion criteria, the number of treatment cycles, and whether or not post-progression treatment was allowed.

Azacitidine and decitabine have also been studied for the treatment of elderly patients with AML. Elderly patients with AML have a poor prognosis with intensive chemotherapy. Because of high therapy-related mortality, less aggressive therapies are often used. A phase III study in elderly patients ( $\geq 65$ years) with AML who were unfit for intensive chemotherapy compared lowdose decitabine with conventional treatments, including low-dose cytarabine and supportive care. The study demonstrated a significant survival benefit for decitabine compared to conventional treatment (7.7 vs. 5.0 months; hazard ratio [HR]: 0.82; 95\% confidence interval [Cl]: 0.68-0.99; $p=.037)$. Decitabine improved the complete remission rate $(17.8 \%$ vs. $7.8 \%$, respectively; $p=.001)$ without hampering treatment safety [6]. Based on these results, in September 2012, decitabine was registered by the European Medicines Agency (EMA) for 
Table 2. Summary table

\begin{tabular}{|c|c|c|}
\hline Generic name & Azacitidine & Decitabine \\
\hline Synonym & 5-azacytidine & 5-aza-2'-deoxycytidine \\
\hline Commercial name & $\begin{array}{l}\text { Vidaza }{ }^{\circledR} \text { (Celgene Europe, } \\
\text { Windsor, U.K.) }\end{array}$ & $\begin{array}{l}\text { Dacogen } ® \text { (MGI Pharma, } \\
\text { Bloomington, MN) }\end{array}$ \\
\hline Molecular weight & $244.2 \mathrm{Da}$ & $228.2 \mathrm{Da}$ \\
\hline Mechanism of action & $\begin{array}{l}\text { Inhibition of DNA } \\
\text { methyltransferase }\end{array}$ & $\begin{array}{l}\text { Inhibition of DNA } \\
\text { methyltransferase }\end{array}$ \\
\hline Cell-cycle specificity & S-phase & S-phase \\
\hline Route of administration & Subcutaneous injection & Intravenous infusion \\
\hline Bioavailability & $89 \%$ & $100 \%$ \\
\hline Metabolism & $\begin{array}{l}\text { Deamination mediated } \\
\text { by cytidine deaminase, } \\
\text { principally found in the liver } \\
\text { but also in granulocytes, } \\
\text { intestinal epithelium and } \\
\text { whole blood. } \\
\text { The metabolism is not } \\
\text { mediated by cytochrome } \\
\text { P450 isoenzymes. }\end{array}$ & As azacitidine \\
\hline Elimination & $\begin{array}{l}\text { Primarily via urine }(50-85 \%) \text {, } \\
\text { small amount via feces }(<1 \%) \text {. }\end{array}$ & Primarily via urine \\
\hline Terminal half-life & About 41 minutes & 37-47 minutes \\
\hline Main toxicities & $\begin{array}{l}\text { As decitabine; } \\
\text { plus, specific for azacitidine, } \\
\text { injection site adverse } \\
\text { reactions, mainly erythema. }\end{array}$ & $\begin{array}{l}\text { Myelosuppression } \\
\text { (mainly neutropenia } \\
\text { and thrombocytopenia); } \\
\text { gastrointestinal toxicities (nausea, } \\
\text { vomiting, diarrhea, constipation). }\end{array}$ \\
\hline Unique features & $\begin{array}{l}\text { Reversal of DNA } \\
\text { hypermethylation }\end{array}$ & $\begin{array}{l}\text { Reversal of DNA } \\
\text { hypermethylation }\end{array}$ \\
\hline Dose adaptations & $\begin{array}{l}\text { Based on hematological } \\
\text { response and toxicity, } \\
\text { a dose delay or reduction } \\
\text { may be necessary. } \\
\text { Renal insufficiency: no } \\
\text { specific modification to the } \\
\text { starting dose recommended, } \\
\text { however monitor closely and } \\
\text { reduce dose if necessary. }\end{array}$ & $\begin{array}{l}\text { Myelosuppression }(\mathrm{ANC}<1,000 / \\
\mu \mathrm{L} \text { and platelets }<50,000 / \mu \mathrm{L}) \text { : } \\
\text { delay of the subsequent cycle and } \\
\text { (in case of the original } 6 \text {-weekly } \\
\text { treatment regimen) temporary } \\
\text { dose reduction. } \\
\text { Renal insufficiency: as azacitidine }\end{array}$ \\
\hline
\end{tabular}

Abbreviation: ANC, absolute neutrophil count. 
the treatment of elderly patients ( $\geq 65$ years) with AML who are not eligible for treatment with standard induction chemotherapy.

An important question is whether therapy with hypomethylating agents in elderly patients with AML may also improve outcome compared with intensive chemotherapy. A retrospective study in 671 elderly patients ( $\geq 65$ years) with newly diagnosed $A M L$ demonstrated that treatment with hypomethylating agents resulted in a lower complete response (CR) rate and overall response rate (ORR) than intensive chemotherapy (CR: $28 \%$ vs. $42 \%$; ORR: $29 \%$ vs. $47 \%$, respectively). However the two-year relapse-free survival rates ( $40 \%$ vs. $30 \%, p=.843$ ) and median survival times (6.5 vs. 6.7 months, $p=.413$ ) were similar in the two groups. This suggests that the long-term outcomes for elderly patients with AML who were treated with hypomethylating agents are similar to the long-term outcomes after intensive chemotherapy [7].

\section{CLINICAL USE}

Azacitidine is available as a white lyophilized powder, which has to be reconstituted with Water for Injection immediately prior to administration to obtain a $25 \mathrm{mg} / \mathrm{mL}$ suspension. The azacitidine suspension should be injected subcutaneously into the upper arm, thigh or abdomen. The site of the injection should be rotated. The recommended starting dose for the first treatment cycle of azacitidine (for all patients regardless of baseline hematology laboratory values) is $75 \mathrm{mg} / \mathrm{m}^{2}$ daily for 7 days, followed by a rest period of 21 days. Cycles should be repeated every 4 weeks. In subsequent treatment cycles, the dose should be adjusted based on hematologic response and toxicity. A delay in starting the next cycle or a dose reduction may be necessary [8].

In addition to this 7-day dosing schedule, alternative azacitidine schedules that omit the weekend doses have been evaluated. Although these regimens showed similar benefits regarding transfusion independence and hematologic improvement, effects on time to AML transformation or overall survival were not compared. Furthermore, the study population consisted primarily of patients with lower-risk MDS [9]. Therefore, it remains unknown whether 
these alternative regimens yield comparable efficacy as the approved 7-day azacitidine schedule.

Decitabine is also available as a white lyophilized powder. It has to be reconstituted with $10 \mathrm{~mL}$ sterile Water for Injection in order to obtain a $5 \mathrm{mg} /$ $\mathrm{mL}$ solution. Immediately after reconstitution, this solution should be further diluted with $0.9 \%$ sodium chloride solution for injection, $5 \%$ glucose solution for injection, or lactated Ringer's solution for injection to a final drug concentration of $0.1-1.0 \mathrm{mg} / \mathrm{mL}[10]$.

At present, two regimens for decitabine administration are approved by the U.S. Food and Drug Administration: the original 6-weekly regimen of $15 \mathrm{mg} / \mathrm{m}^{2}$ administered by continuous intravenous infusion over 3 hours repeated every 8 hours for 3 days, and the later approved 4 -weekly regimen of $20 \mathrm{mg} / \mathrm{m}^{2}$ by continuous intravenous infusion over 1 hour repeated daily for 5 days [10]. The main advantage of this new regimen is the reduced infusion time, which enables treatment in the outpatient setting [11]. This 4-weekly decitabine regimen is also the dose scheme recently approved by the EMA for treatment of elderly patients ( $\geq 65$ years) with AML who are not eligible for standard induction chemotherapy [6].

Complete blood counts should be determined at least prior to each cycle to monitor response and toxicity. If myelosuppression is present (absolute neutrophil count $<1,000 / \mu \mathrm{L}$ and platelets $<50,000 / \mu \mathrm{L}$ ), subsequent decitabine cycles should be delayed; for the original 6-weekly treatment regimen, the dose should be temporarily reduced [10].

\section{DURATION OF TREATMENT}

Azacitidine treatment should be continued for a minimum of six courses before evaluating the effects of treatment. Response to treatment is assessed according to the International Working Group criteria, distinguishing three response categories: complete response, partial response, and hematological improvement [12]. Silverman et al. found that in $91 \%$ of the responding 
patients, initial response was seen within six cycles [13]. Continued azacitidine treatment after initial response further improved the response in $48 \%$ of patients. Maximum response was achieved by $92 \%$ of responders by cycle 12 [13]. Similarly for decitabine, a broad range was found for the time to initial response. The median time to response was more than three months $[4,5]$. It is recommended to continue decitabine treatment for a minimum of four cycles (i.e., six months), provided that patients are monitored for hematologic and renal toxicities [10]. Treatment with azacitidine or decitabine should be continued as long as the patient shows continued benefit $[8,10]$. The median duration of the hematological response was about 13 months in both azacitidine studies $[2,14,15]$ and $9-10$ months in both decitabine studies $[4,5]$.

The possibility of retreatment with decitabine at the moment of disease recurrence after initial treatment was investigated. Retreatment with decitabine was found to result in objective responses in $45 \%$ of the patients who previously responded to decitabine. However, the quality and duration of the second disease remission were found to be inferior. Therefore, patients who respond to decitabine might possibly derive more clinical benefit from continuation of the initial treatment [16].

\section{MECHANISM OF ACTION}

Epigenetic changes, such as aberrant DNA methylation, have an important place in the pathogenesis of MDS and AML. The most studied change of DNA methylation is the silencing of tumor suppressor genes by hypermethylation of the CpG islands within the promoter region [17]. In contrast to structural changes such as mutation or deletion causing permanent loss of gene expression, epigenetic changes can be pharmacologically reversed, resulting in gene re-expression and restoration of normal cellular functions [18].

Azacitidine and decitabine (5-aza-2'-deoxycytidine) are cytidine analogs in which the carbon atom at position 5 in the pyrimidine ring has been replaced by a nitrogen atom (Fig. 1). Originally, they were intended as cytotoxic drugs. However, it was discovered that a low dose of these drugs could cause DNA 


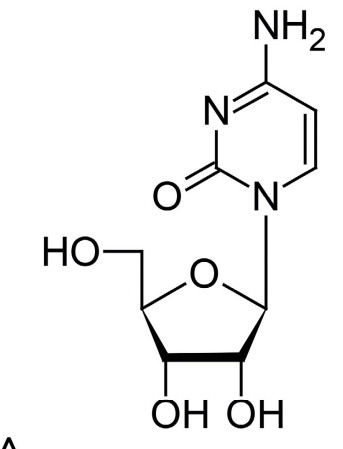

A.

B.<smiles>Nc1ncn(C2OC3C(O)C(CO)OC32)c(=O)n1</smiles>

C.<smiles>Nc1ncn(C2C[C@H](O)[C@H](CO)O2)c(=O)n1</smiles>

Figure 1. Chemical structures of cytidine (A), azacitidine (B) and decitabine (5-aza-2'deoxycytidine) (C).

demethylation by inactivation of DNA methyltransferase-1 (DNMT-1), the enzyme responsible for methylation of the DNA [19].

Following cellular uptake, azacitidine and decitabine are converted into their monophosphates, diphosphates, and triphosphates. Decitabine triphosphate is a deoxyribonucleotide that is incorporated only into DNA. Azacitidine is mainly converted to azacitidine triphosphate, which is incorporated into the RNA. A smaller portion of the administered azacitidine, about $10 \%-20 \%$, is converted to 5-aza-2'-deoxycytidine triphosphate via the enzyme ribonucleotide reductase and is available for incorporation into the DNA. Incorporation into the DNA results in the formation of adducts between the DNA and DNMT-1. At high doses, the DNA is not able to recover and cell death occurs. However, at lower doses the formed adducts are degraded by the proteosome, after which the DNA is restored. DNA synthesis is then resumed in the absence of DNMT1. As a consequence the aberrant DNA methylation pattern can no longer be reproduced toward the daughter strands [20]. In this way, a low dose of azacitidine or decitabine is able to induce re-expression of previously silenced genes. Reactivation of cell cycle-regulating genes that were initially silenced due to hypermethylation may induce cell differentiation, reduce proliferation, and/ or increase apoptosis of the daughter cells [21]. 


\section{RESPONSE PREDICTION}

Several reports have dealt with response prediction, focusing on two different outcomes: response to treatment with hypomethylating agents and longer-term endpoints (mainly overall survival). For patients with higher-risk MDS, several factors were associated with a slightly superior response to hypomethylating therapy: no prior therapy, shorter MDS duration [22], longer MDS duration prior to treatment [5], normal karyotype, a bone marrow blast percentage $<15 \%$, and no previous exposure to low-dose cytarabine [23].

For prediction of the overall survival following azacitidine therapy, Itzykson et al. proposed a three-category prognostic classification system based on independent prognostic factors. Predictors for a favorable overall survival were a performance status $<2$, high-risk cytogenetics, absence of circulating blasts, and no red blood cell transfusion dependency ( $<4$ units per 8 weeks). The prognostic score, based on these four factors, discriminated three groups with median overall survival times of 6,15 , and $>26$ months, respectively [23]. The obtained prognostic score was validated in several independent patient cohorts receiving azacitidine $[24,25]$. Moreover, platelet doubling after the first cycle of azacitidine was found to predict a longer overall survival time for patients with MDS, CMML, or AML [25].

It is nevertheless important to further improve identification of prognostic factors of response to hypomethylating agents. Mutations in the TET2 and DNMT3A genes (both regulating DNA methylation) and the ASXL1 gene (regulating histone modifications) are associated with a favorable response of patients with higher-risk MDS to azacitidine treatment. However, these genetic factors have not yet been clearly associated with differences in survival $[26,27]$.

\section{TREATMENT FAILURE}

Only about $50 \%$ of patients respond to therapy with hypomethylating agents, and the majority of the responders relapse within 2 years [2]. The outcome after failure of hypomethylating therapy is poor. Retrospective analysis of the 
outcome of 435 patients with higher-risk MDS who experienced azacitidine treatment failure demonstrated a median overall survival of 5.6 months and a 2 -year survival probability of $15 \%$ [28]. For 87 patients with MDS or CMML, a median overall survival time of 4.3 months was reported after decitabine failure, with an estimated 1-year survival rate of $28 \%$ [29]. The outcome of patients with secondary AML arising from MDS after azacitidine failure was even worse, with a median overall survival time of 3.6 months and a 1-year survival rate of only $8 \%$ [30]. There is no standard of care for salvage therapy after failure of hypomethylating therapy. Because of the limited therapeutic options, this is a challenging cohort of patients in a setting that constitutes an important area of research.

One might question whether it could be useful to switch from one hypomethylating agent to the other after treatment failure. Considering the slightly different mechanisms of action of azacitidine and decitabine, as well as the distinct metabolic activation routes concerned, there is not necessarily complete cross-resistance between both hypomethylating agents. Failure of one drug may not exclude activity of the other. A small study investigated the value of decitabine treatment after azacitidine failure [31]. Of the 14 patients with MDS who were analyzed, three patients achieved complete remission and one patient showed hematologic improvement, which corresponded to an overall response rate of $28 \%$. Of the responders, prior azacitidine therapy was stopped because of disease progression ( $n=1)$, lack of response $(n=2)$, and severe skin toxicity $(n=1)$ [31], which suggests that changing hypomethylating therapy from azacitidine to decitabine may have some clinical utility. No studies were found investigating a switch in the opposite direction (i.e., the use of azacitidine in decitabine failures).

\section{COMBINATION THERAPIES}

Numerous reports have dealt with the combination of hypomethylating agents and other agents, ranging from growth factors to histone deacetylase inhibitors. A fully comprehensive overview of all combination strategies currently investigated is beyond the scope of this review. However, we would 
like to highlight the promising combination of a hypomethylating agent with the immunomodulating drug lenalidomide. A phase II study demonstrated that the combination of azacitidine $\left(75 \mathrm{mg} / \mathrm{m}^{2}\right.$ daily for 5 days) and lenalidomide $(10 \mathrm{mg} /$ day for 21 days, 28-day cycle) was well tolerated and highly active in patients with higher-risk MDS. Using this combination, $44 \%$ of patients achieved CR and the ORR was 72\% [32]. These results seem to be better than the response rates obtained with azacitidine monotherapy in the AZA-001 trial (CR: 17\%, ORR: 49\%) [2], although the discrepant response rates might be attributable to differences between the study populations. Randomized trials comparing this combination regimen, as well as others, with azacitidine monotherapy, are currently ongoing.

\section{BIOANALYSIS}

The bioanalysis of azacitidine and decitabine in human plasma can be exerted by high-performance liquid chromatography coupled with tandem mass spectrometry detection $[33,34]$.

\section{PHARMACOKINETICS}

\section{Absorption}

Azacitidine is rapidly absorbed after subcutaneous administration. Following a standard dose of $75 \mathrm{mg} / \mathrm{m}^{2}$ subcutaneously, maximum plasma concentration $(750 \pm 403 \mathrm{ng} / \mathrm{mL})$ is reached after 30 minutes. The absolute bioavailability of azacitidine after subcutaneous administration is approximately $89 \%$ compared to intravenous infusion [35].

For decitabine, maximum plasma concentration is generally observed at the end of infusion. Following a 3-hour intravenous infusion of $15 \mathrm{mg} / \mathrm{m}^{2}$, the maximum plasma concentration is $64.8-77.0 \mathrm{ng} / \mathrm{mL}$. At this time, steady-state plasma concentration is reached as well [36]. 


\section{Distribution}

Both azacitidine and decitabine are widely distributed over tissues. For azacitidine, the mean volume of distribution after intravenous administration was about $76 \mathrm{~L}$ [35]. For decitabine, a volume of distribution of $63-89 \mathrm{~L} / \mathrm{m}^{2}$ was found at steady-state [36].

\section{Metabolism}

Intracellular phosphorylation is a pivotal step for azacitidine and decitabine to become active. Beyond this step, the exact metabolic fate of azacitidine and decitabine is unknown. Both drugs undergo spontaneous hydrolysis in aqueous solution and deamination mediated by cytidine deaminase, which is principally found in the liver but also in granulocytes, intestinal epithelium, and whole blood. In vitro studies indicate that the metabolism of azacitidine and decitabine is not mediated by cytochrome P450 isoenzymes. Clinically significant inhibitory or inductive effects on CYP enzymes are also unlikely [10,37].

\section{Elimination}

Azacitidine is cleared rapidly from plasma. The mean elimination half-life after a standard subcutaneous dose of $75 \mathrm{mg} / \mathrm{m}^{2}$ is about 41 minutes. This is approximately twofold higher than the mean elimination half-life after intravenous administration (22 minutes). The systemic clearance of azacitidine is $147 \pm 47 \mathrm{~L} / \mathrm{h}$ [35]. Urinary excretion is the primary route of elimination of azacitidine and its metabolites. Following intravenous and subcutaneous administration of ${ }^{14} \mathrm{C}$-labeled azacitidine, $50 \%-85 \%$ of the administered radioactivity was recovered in urine, whereas less than $1 \%$ was recovered in the feces. Unfortunately, parent drug and possible drug metabolites could not be distinguished in these ${ }^{14} \mathrm{C}$-azacitidine studies. Nevertheless, the longer half-life calculated based on ${ }^{14} \mathrm{C}$ radioactivity (3.4-6.2 hours) suggested the presence of circulating metabolites [38,39].

The terminal elimination half-life $\left(t_{1 / 2}\right)$ of decitabine is 37-47 minutes. Consequently, steady-state in plasma is reached at the end of the 3-hour infusion. Thereafter, plasma concentrations decline biexponentially and are measurable up to 2 hours post-infusion. The total body clearance of decitabine 
is $125-132 \mathrm{~L} / \mathrm{h} / \mathrm{m}^{2}$. Decitabine plasma pharmacokinetics remained unchanged upon repeated dosing and even from cycle to cycle [36].

\section{Alterations for special populations}

Because azacitidine, decitabine, and/or their metabolites are primarily excreted by the kidneys, caution is needed in patients with renal impairment. Liver function tests and serum creatinine should be determined prior to initiation of therapy and prior to each treatment cycle. No specific modification to the starting dose is recommended in patients with renal insufficiency prior to starting treatment. However, patients should be closely monitored for toxicity and dose reductions should be implemented if necessary [8]. To our knowledge, there has only been one retrospective study that examined the feasibility of therapy with azacitidine or decitabine in patients with renal insufficiency (creatinine clearance $\leq 59 \mathrm{~mL} / \mathrm{min}$ ) [40]. Toxicity rates were comparable to those in previous reports in patients with adequate renal function. Most patients were able to tolerate the hypomethylating agents at standard doses. However, dose reductions and treatment interruptions were required for some patients, particularly those with severe renal insufficiency (i.e., creatinine clearance $\leq$ $30 \mathrm{~mL} / \mathrm{min}$ ). This group experienced more treatment-related toxicities, mainly myelosuppression and worsening of the renal function [40]. A prospective study may be warranted to better define the pharmacokinetics of hypomethylating agents in patients with renal insufficiency and to determine if dose adjustments at the beginning of treatment would be needed. One trial (NCT00652626) is currently recruiting patients to study the effect of renal impairment on the pharmacokinetics of azacitidine.

\section{TOXICITY}

Azacitidine and decitabine are generally well tolerated and have a manageable toxicity profile. The most common toxicity is myelosuppression, mainly displaying as neutropenia and thrombocytopenia. However, toxicity can be difficult to assess for patients with severe cytopenias from their disease [2,14,41]. Adverse events related to myelosuppression typically occur in the third week of the 
treatment cycle. Most patients achieve hematologic recovery prior to the next treatment cycle. Otherwise, a delay in starting the next cycle or a dose reduction may be necessary. Hematologic adverse events were most frequently observed during the first two treatment cycles and nadir values for hematologic parameters generally improved during subsequent cycles [4,41]. Data about an elevated risk for infections or bleeding caused by hypomethylating agents were not very consistent [41]. Patients should be advised to promptly report febrile episodes and to be observant for signs and symptoms of bleeding [8].

The most common nonhematological adverse events were gastrointestinal toxicities such as nausea, vomiting, diarrhea, and constipation, which generally occurred in the first week of the treatment cycle. These events were generally mild and transient. They could be managed with concomitant medications, including antiemetics and antidiarrheals $[2,4]$.

Azacitidine administration can result in adverse reactions at the site of injection, mainly erythema. Most of these reactions are transient. A minority of patients $(<12 \%)$ required treatment with corticosteroids and/or antihistamines. The incidence of adverse reactions at the site of injection might be reduced by changing the needle used to load the syringe to a clean needle (i.e., without azacitidine residue) before injection. Additionally, use of warm compresses after injection may alleviate symptoms [41].

\section{CONTRAINDICATIONS OR SPECIAL PRECAUTIONS}

According to the product information, azacitidine is contraindicated in patients with advanced malignant hepatic tumors. There are no adequate data on the use of azacitidine or decitabine in pregnant women. Studies in mice have shown reproductive toxicity. Based on these results and their mechanism of action, azacitidine and decitabine should not be used during pregnancy, especially during the first trimester, unless clearly necessary. Men and women of childbearing potential must use effective contraception during and up to 3 months after treatment. It is not known whether azacitidine, decitabine, or their metabolites are excreted in human milk. Because of potential serious adverse 
reactions in nursing infants, breastfeeding is contraindicated during treatment. The safety and effectiveness in children younger than 18 years have not been established $[8,10]$.

\section{CLINICAL MONITORING}

Complete blood counts should be performed at least prior to each cycle to monitor response and toxicity. Possible consequences for the administered dose were previously described. Liver function tests and serum creatinine should be determined prior to initiation of therapy and prior to each treatment cycle. No dose reduction is recommended for patients with hepatic or renal insufficiency prior to starting treatment, but patients should be closely monitored for toxicity $[8,10]$. For patients treated with azacitidine, the serum bicarbonate concentrations should be monitored. If unexplained reductions in serum bicarbonate ( $<20 \mathrm{mmol} / \mathrm{L}$ ) or elevations of serum creatinine or blood urea nitrogen (at least twofold above baseline and above the upper limit of normal) occur, the dose should be reduced or administration delayed [8]. Decitabine treatment should be postponed if any of the following nonhematologic toxicities are present: serum creatinine $\geq 177 \mu \mathrm{mol} / \mathrm{L}(2 \mathrm{mg} / \mathrm{dL}$ ); alanine aminotransferase or total bilirubin at least two times the upper limit of normal; or active or uncontrolled infection. Decitabine treatment should not be restarted until the toxicity is resolved [10]. 


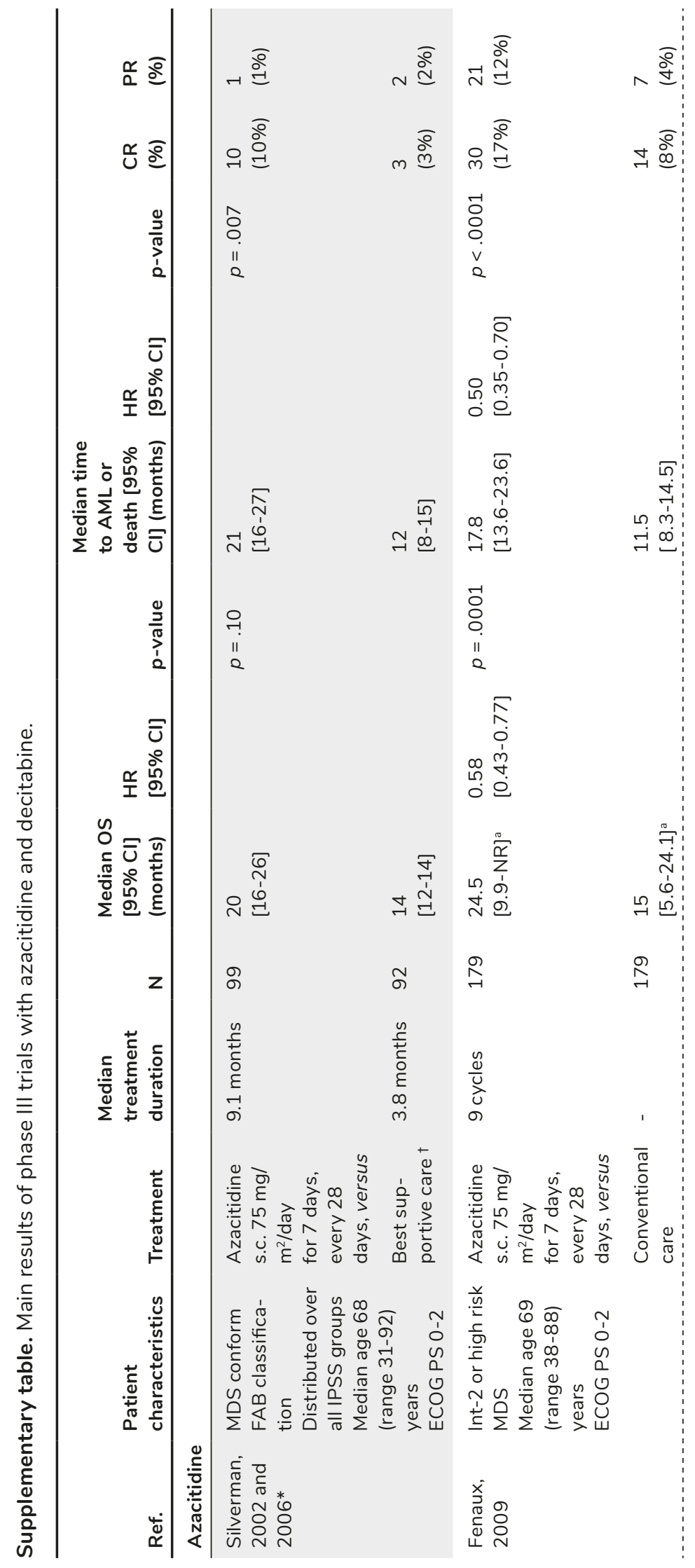




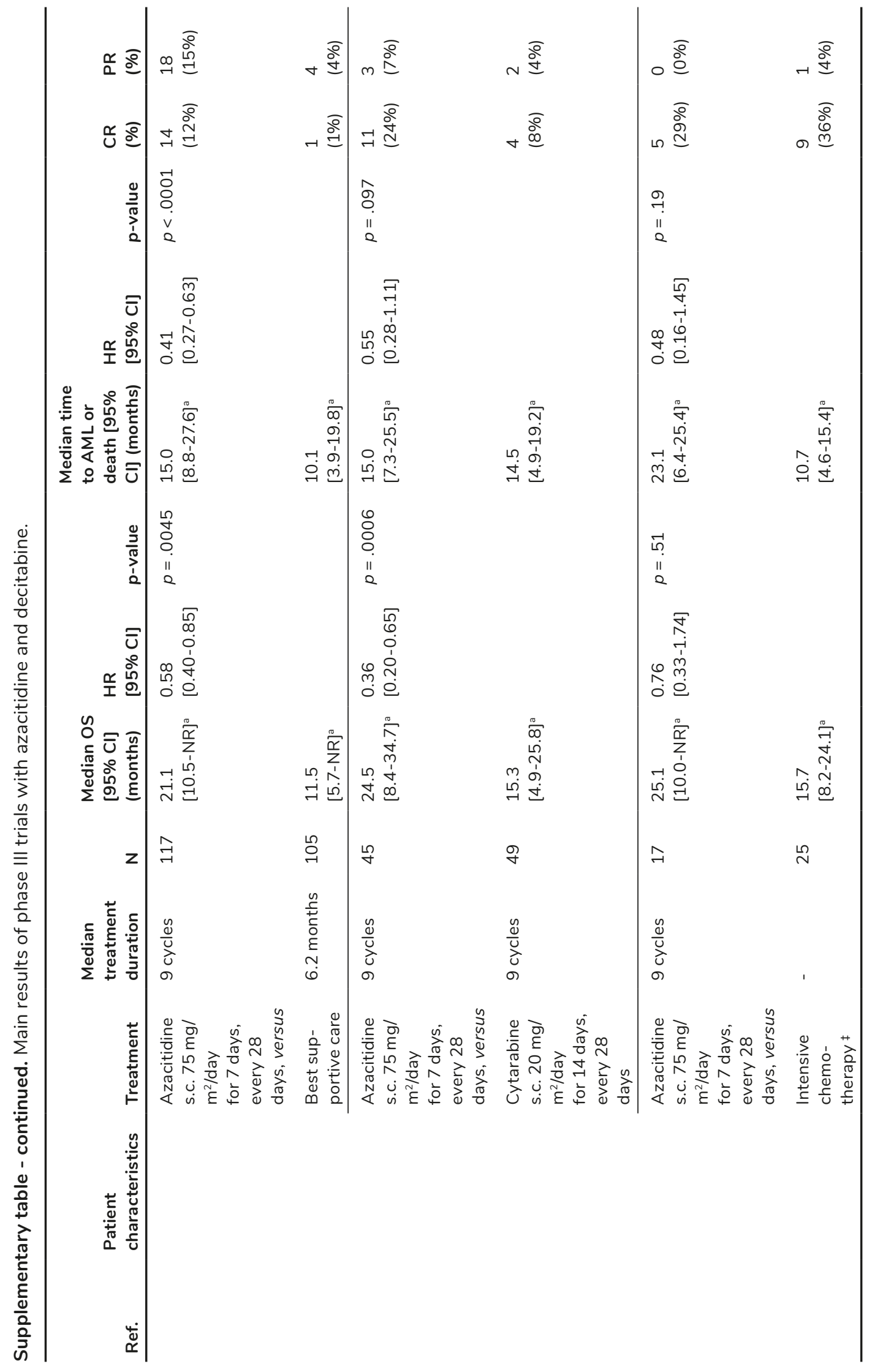




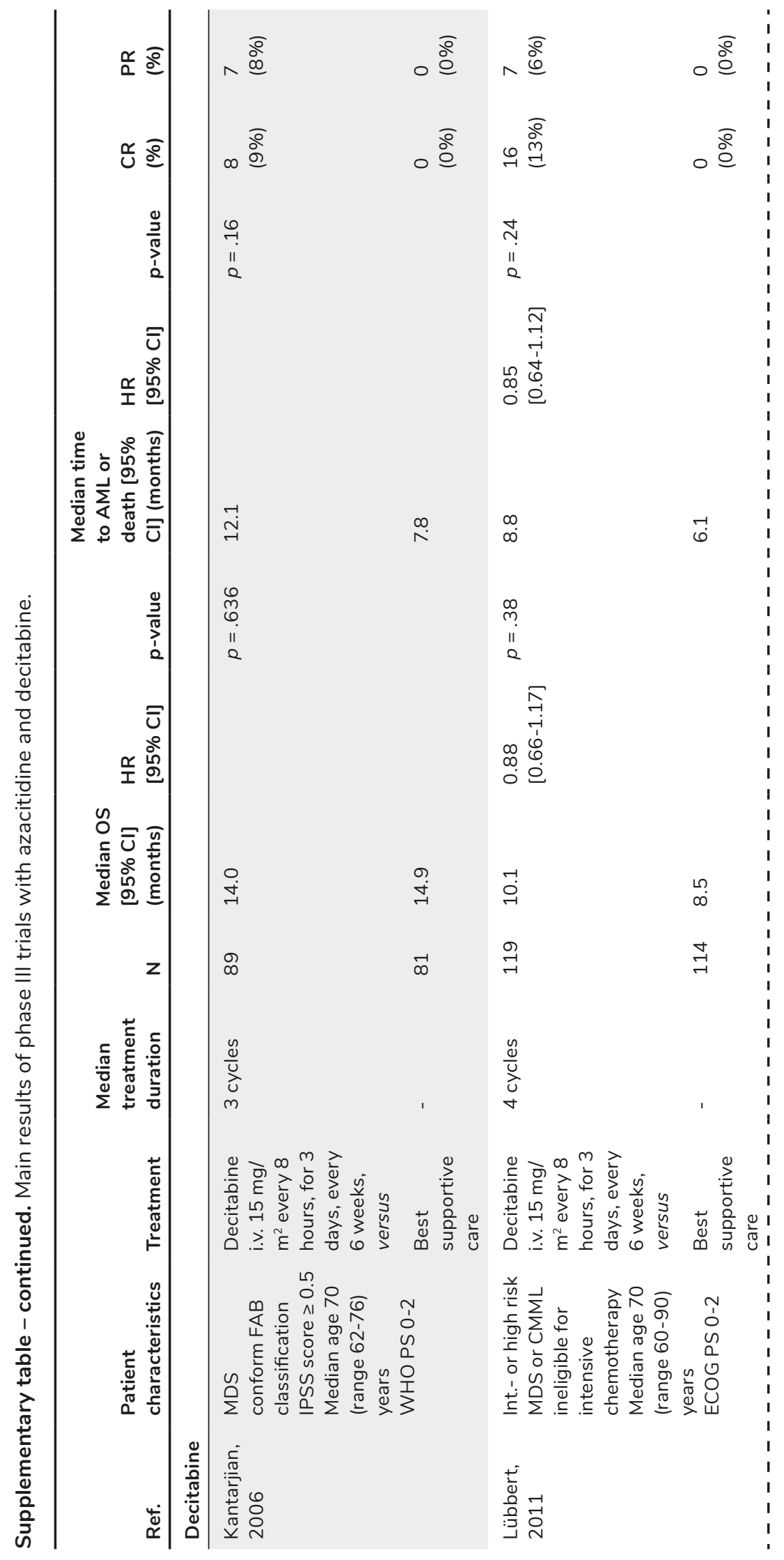




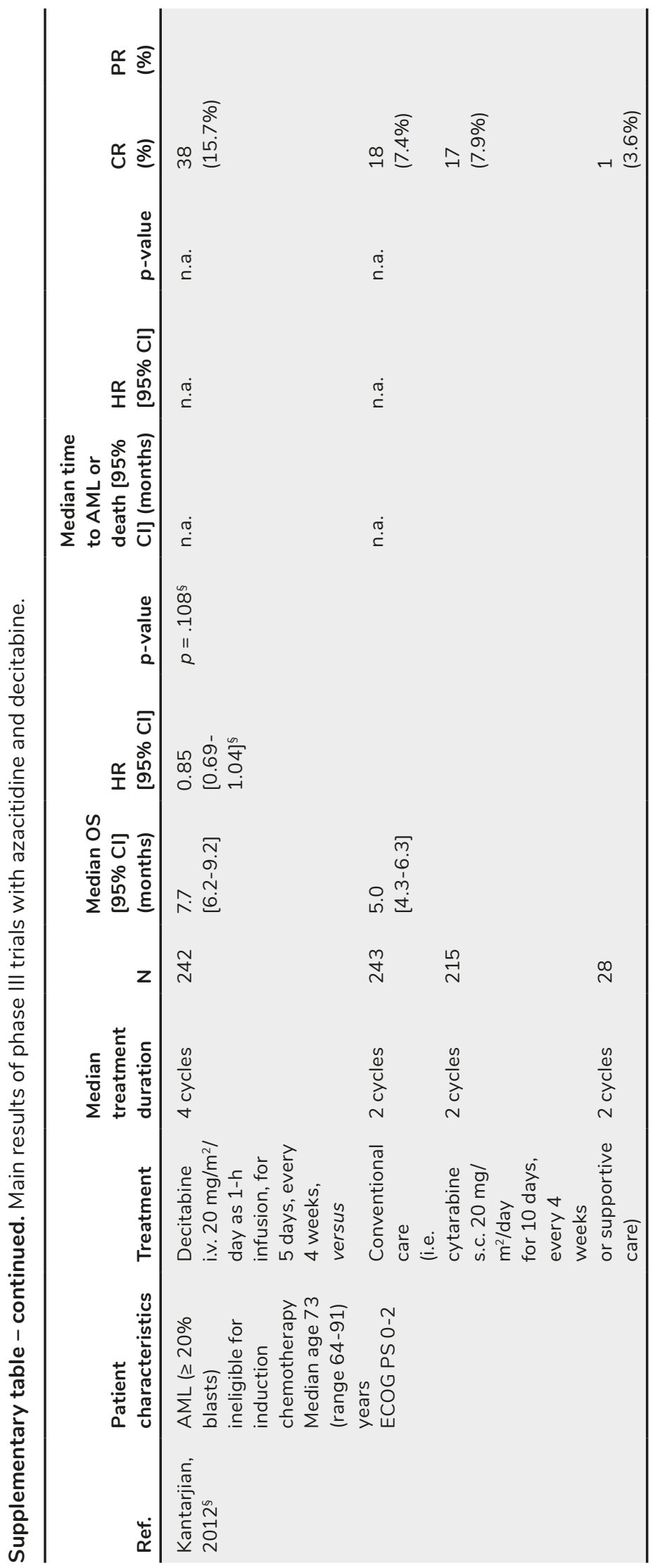

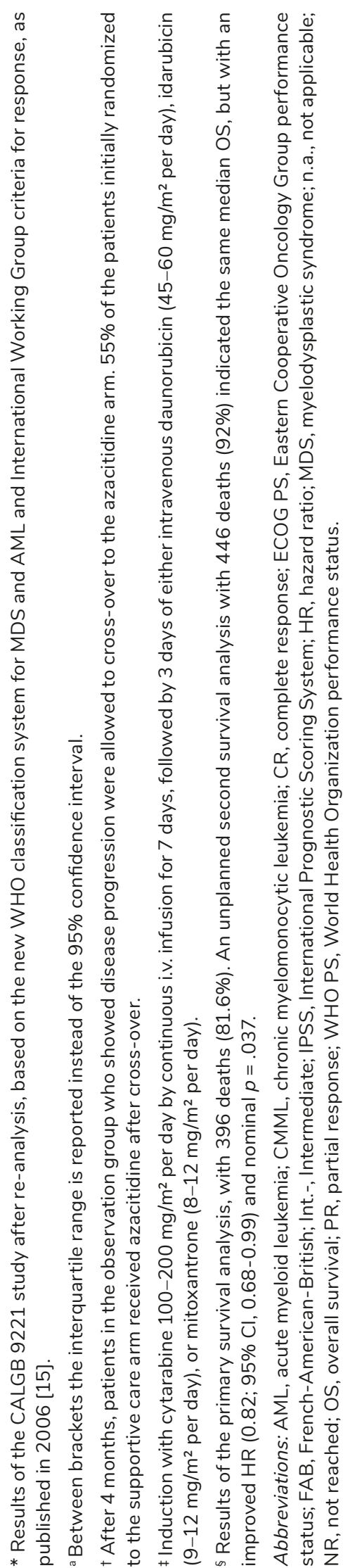




\section{REFERENCES}

[1] Beran M. Intensive chemotherapy for patients with high-risk myelodysplastic syndrome. Int J Hematol 2000;72(2):139-150.

[2] Fenaux P, Mufti GJ, Hellstrom-Lindberg E et al. Efficacy of azacitidine compared with that of conventional care regimens in the treatment of higher-risk myelodysplastic syndromes: a randomized, open-label, phase III study. Lancet Oncol 2009;10(3):223-232.

[3] Itzykson R, Fenaux P. Optimizing hypomethylating agents in myelodysplastic syndromes. Curr Opin Hematol 2012;19(2):65-70.

[4] Kantarjian H, Issa JP, Rosenfeld CS, et al. Decitabine improves patient outcomes in myelodysplastic syndromes: results of a phase III randomized study. Cancer 2006;106(8):1794-1803.

[5] Lübbert M, Suciu S, Baila L, et al. Low-Dose Decitabine Versus Best Supportive Care in Elderly Patients With Intermediate- or High-Risk Myelodysplastic Syndrome (MDS) Ineligible for Intensive Chemotherapy: Final Results of the Randomized Phase III Study of the European Organization for Research and Treatment of Cancer Leukemia Group and the German MDS Study Group. J Clin Oncol 2011;29(15):1987-1996.

[6] Kantarjian HM, Thomas XG, Dmoszynska A, et al. Multicenter, Randomized, Open-Label, Phase III Trial of Decitabine Versus Patient Choice, With Physician Advice, of Either Supportive Care or Low-Dose Cytarabine for the Treatment of Older Patients With Newly Diagnosed Acute Myeloid Leukemia. J Clin Oncol 2012;30(21):2670-2677.

[7] Quintás-Cardama A, Ravandi F, Liu-Dumlao $\mathrm{T}$, et al. Epigenetic therapy is associated with similar survival compared with intensive chemotherapy in older patients with newly diagnosed acute myeloid leukemia. Blood 2012;120(24):4840-4845.

[8] Summary of Product Characteristics Vidaza $25 \mathrm{mg} / \mathrm{ml}$ powder for suspension for injection; http://www.ema.europa.eu/docs. Accessed September 11, 2012.

[9] Lyons RM, Cosgriff TM, Modi SS, et al. Hematologic response to three alternative dosing schedules of azacitidine in patients with myelodysplastic syndromes. J Clin Oncol 2009;27(11):1850-1856.
[10] Approved labeling Dacogen (decitabine) for injection dated 2/5/2006; www.accessdata. fda.gov

[11] Steensma DP, Baer MR, Slack JL, et al. Multicenter study of decitabine administered daily for 5 days every 4 weeks to adults with myelodysplastic syndromes: the alternative dosing for outpatient treatment (ADOPT) trial. J Clin Oncol 2009;27(23):3842-3848.

[12] Cheson BD, Greenberg PL, Bennett JM, et al. Clinical application and proposal for modification of the International Working Group (IWG) response criteria in myelodysplasia. Blood 2006;108(2):419-425.

[13] Silverman LR, Fenaux P, Mufti GJ, et al. Continued azacitidine therapy beyond time of first response improves quality of response in patients with higher-risk myelodysplastic syndromes. Cancer 2011;117(12):2697-2702.

[14] Silverman LR, Demakos EP, Peterson BL, et al. Randomized controlled trial of azacitidine in patients with the myelodysplastic syndrome: a study of the cancer and leukemia group B. $J$ Clin Oncol 2002;20(10):2429-2440.

[15] Silverman LR, McKenzie DR, Peterson BL, et al. Further analysis of trials with azacitidine in patients with myelodysplastic syndrome: studies 8421,8921 , and 9221 by the Cancer and Leukemia Group B. J Clin Oncol 2006;24(24):3895-3903.

[16] Rüter $B$, Wijermans $P W$, Lübbert $M$. Superiority of prolonged low-dose azanucleoside administration? Results of 5-aza-2'-deoxycytidine retreatment in high-risk myelodysplasia patients. Cancer 2006;106(8):1744-1750.

[17] Issa JP. Epigenetic changes in the myelodysplastic syndrome. Hematol Oncol Clin North Am 2010;24(2):317-330.

[18] Issa JP, Kantarjian HM. Targeting DNA methylation. Clin Cancer Res 2009;15(12):3938-3946.

[19] Stresemann C, Lyko F. Modes of action of the DNA methyltransferase inhibitors azacytidine and decitabine. Int J Cancer 2008;123(1):8-13.

[20] McCabe MT, Brandes JC, Vertino PM. Cancer DNA methylation: molecular mechanisms and clinical implications. Clin Cancer Res 2009;15(12):3927-3937. 
[21] Christman JK. 5-Azacytidine and 5-aza2'-deoxycytidine as inhibitors of DNA methylation: mechanistic studies and their implications for cancer therapy. Oncogene 2002;21(35):5483-5495.

[22] Kantarjian HM, O'Brien S, Shan J, et al. Update of the decitabine experience in higher risk myelodysplastic syndrome and analysis of prognostic factors associated with outcome. Cancer 2007; 109(2):265-273.

[23] Itzykson R, Thépot S, Quesnel B, et al. Prognostic factors for response and overall survival in 282 patients with higher-risk myelodysplastic syndromes treated with azacitidine. Blood 2011;117(2):403-411.

[24] Breccia M, Loglisci G, Cannella L, et al. Application of French prognostic score to patients with International Prognostic Scoring System intermediate-2 or high risk myelodysplastic syndromes treated with 5 -azacitidine is able to evaluate overall survival and rate of response. Leuk Lymphoma 2012;53:982-983.

[25] van der Helm LH, Alhan C, Wijermans PW, et al. Platelet doubling after the first azacitidine cycle is a promising predictor for response in myelodysplastic syndromes (MDS), chronic myelomonocytic leukaemia (CMML) and acute myeloid leukaemia (AML) patients in the Dutch azacitidine compassionate named patient programme. Br J Haematol 2011;155:599- 606.

[26] Itzykson R, Kosmider O, Cluzeau T, et al. Impact of TET2 mutations on response rate to azacitidine in myelodysplastic syndromes and low blast count acute myeloid leukemias. Leukemia 2011;25:1147-1152.

[27] Itzykson R, Fenaux P. Predicting the outcome of patients with higher-risk myelodysplastic syndrome treated with hypomethylating agents. Leuk Lymphoma 2012;53(5):760-762.

[28] Prébet T, Gore SD, Esterni B, et al. Outcome of high-risk myelodysplastic syndrome after azacitidine treatment failure. J Clin Oncol 2011;29:3322-3327.

[29] Jabbour E, Garcia-Manero G, Batty N, et al. Outcome of patients with myelodysplastic syndrome after failure of decitabine therapy. Cancer 2010;116:3830-3834.

[30] Prébet T, Gore SD, Thépot S, et al. Outcome of acute myeloid leukaemia following myelodysplastic syndrome after azacitidine treatment failure. Br J Haematol 2012;157(6):764-766.
[31] Borthakur G, Ahdab SE, Ravandi F, et al. Activity of decitabine in patients with myelodysplastic syndrome previously treated with azacitidine. Leuk Lymphoma 2008;49:690-695.

[32] Sekeres MA, Tiu RV, Komrokji R, et al. Phase 2 study of the lenalidomide and azacitidine combination in patients with higherrisk myelodysplastic syndromes. Blood 2012;120(25):4945-4951.

[33] Zhao M, Rudek MA, He P, et al. Quantification of 5-azacytidine in plasma by electrospray tandem mass spectrometry coupled with high-performance liquid chromatography. J Chromatogr B Analyt Technol Biomed Life Sci 2004;813(1-2):81-88.

[34] Patel K, Guichard SM, Jodrell DI. Simultaneous determination of decitabine and vorinostat (Suberoylanalide hydroxamic acid, SAHA) by liquid chromatography tandem mass spectrometry for clinical studies. J Chromatogr B Analyt Technol Biomed Life Sci. 2008;863(1):19-25.

[35] Marcucci G, Silverman L, Eller M, et al. Bioavailability of azacitidine subcutaneous versus intravenous in patients with the myelodysplastic syndromes. J Clin Pharmacol 2005;45(5):597-602.

[36] Cashen AF, Shah AK, Todt L, et al. Pharmacokinetics of decitabine administered as a 3-h infusion to patients with acute myeloid leukemia (AML) or myelodysplastic syndrome (MDS). Cancer Chemother Pharmacol 2008;61(5):759-766.

[37] Chen Y, Liu L, Laille E, et al. In vitro assessment of cytochrome P450 inhibition and induction potential of azacitidine. Cancer Chemother Pharmacol 2010;65(5):995-1000.

[38] Troetel WM, Weiss AJ, Stambaugh JE, et al. Absorption, distribution, and excretion of 5-azacytidine (NSC-102816) in man. Cancer Chemother Rep 1972;56(3):405-411.

[39] Israili ZH, Vogler WR, Mingioli ES, et al. The disposition and pharmacokinetics in humans of 5-azacytidine administered intravenously as a bolus or by continuous infusion. Cancer Res 1976;36(4):1453-1461.

[40] Batty GN, Kantarjian H, Issa JP, et al. Feasibility of therapy with hypomethylating agents in patients with renal insufficiency. Clin Lymphoma Myeloma Leuk 2010;10(3):205210. 
[41] Santini V, Fenaux P, Mufti GJ, et al. Management and supportive care measures for adverse events in patients with myelodysplastic syndromes treated with azacitidine. Eur J Haematol 2010;85(2):130138. 


\section{CHAPTER 2.2}

Quantitative determination of azacitidine triphosphate in peripheral blood mononuclear cells using liquid chromatography coupled with high-resolution mass spectrometry

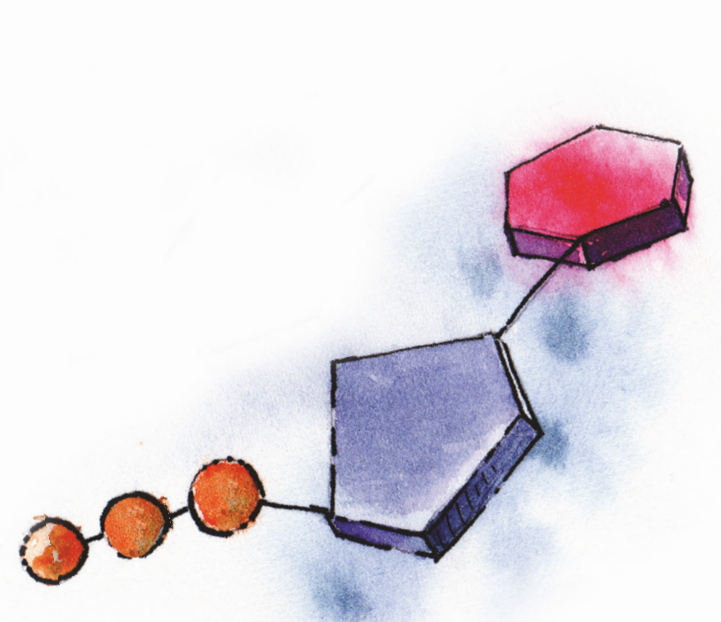

Ellen J.B. Derissen

Michel J.X. Hillebrand Hilde Rosing Hans-Martin M.B. Otten Eric Laille Jan H.M. Schellens Jos H. Beijnen 


\section{ABSTRACT}

Azacitidine is a cytidine analog used in the treatment of myelodysplastic syndromes, chronic myelomonocytic leukemia and acute myeloid leukemia. The pharmacological effect of azacitidine arises after incorporation into the DNA and RNA. To this end, the drug first has to be converted into its triphosphate forms. This paper describes the development of an assay for quantitative determination of azacitidine triphosphate (aza-CTP) in peripheral blood mononuclear cells (PBMCs).

To quantify aza-CTP, separation from the endogenous nucleotides cytidine triphosphate (CTP) and uridine triphosphate (UTP) is required. This was a challenge as the structures of these nucleotides are highly similar and the monoisotopic molecular masses of aza-CTP, UTP and the naturally occurring $\left[{ }^{13} \mathrm{C}\right]$ - and $\left[{ }^{15} \mathrm{~N}\right]$-isotopes of CTP differ less than $0.02 \mathrm{Da}$. Efforts to select a specific $\mathrm{MS}^{2}$-fragment for aza-CTP using a triple quadrupole mass spectrometer remained without success. Therefore, we investigated the feasibility to separate these highly resembling nucleotides based on accurate mass spectrometry using a linear trap quadrupole (LTQ) coupled with an Orbitrap.

The LTQ-Orbitrap was able to differentiate between aza-CTP and the endogenous nucleotides UTP and $\left[{ }^{13} \mathrm{C}\right]$-CTP. There was no baseline resolution between aza-CTP and $\left[{ }^{15} \mathrm{~N}\right]-\mathrm{CTP}$, but the $\left[{ }^{15} \mathrm{~N}\right]-\mathrm{CTP}$ interference was low. For quantification, extracted ion chromatograms were obtained for the accurate $\mathrm{m} / \mathrm{z}$ window of the aza-CTP product ion. The assay was able to determine aza-CTP concentrations in PBMC lysate from 40.7 to $281 \mathrm{nM}$. Assuming that an average cell suspension extracted from $16 \mathrm{~mL}$ blood contains 10 to 42 million PBMCs per $\mathrm{mL}$, this range corresponds with 2.58/10.9-17.8/74.9 pmol aza-CTP per million PBMCs. Intra-assay accuracies were between -1.1 and $9.5 \%$ deviation and coefficient of variation values were $\leq 13.2 \%$.

The assay was successfully applied to quantify aza-CTP in samples from two patients treated with azacitidine. Aza-CTP concentrations up to 19.0 pmol per million PBMCs were measured. This is the first time that aza-CTP concentrations were quantified in PBMCs from patients treated with azacitidine. 
Keywords: azacitidine triphosphate; nucleotides; PBMCs; liquid chromatography-high-resolution mass spectrometry (LC-HRMS); LTQ-Orbitrap 


\section{INTRODUCTION}

The introduction of azacitidine (Vidaza ${ }^{\circledR}$ ) has been a major step forward in the treatment of myelodysplastic syndromes, chronic myelomonocytic leukemia and acute myeloid leukemia [1]. Azacitidine is a cytidine analog in which the carbon atom at position 5 of the pyrimidine ring has been replaced by a nitrogen atom. The effect of azacitidine arises after incorporation into the DNA and RNA. Azacitidine is mainly incorporated in the RNA, where it disrupts RNA and protein metabolism, and to a lesser extent in the DNA where it causes inhibition of DNA methyltransferase-1 [2]. This is the enzyme which is responsible for methylation of the DNA. By inhibition of this enzyme, aberrant DNA methylation patterns, which play an important role in the pathogenesis of the mentioned hematologic abnormalities, will no longer be reproduced toward the daughter cells $[3,4]$.

Before azacitidine can be incorporated into the RNA or DNA, it has to be transported into the cell where it should be converted to one of its triphosphate forms: azacitidine 5'-triphosphate (aza-CTP) or 5-aza-2'-deoxycytidine 5'-triphosphate (aza-dCTP) (Fig. 1) [5]. It is crucial to quantify these intracellular aza-CTP and aza-dCTP levels, because they represent the amount of 'activated' drug available for RNA and DNA incorporation. Understanding the intracellular pharmacokinetics can help to improve the applied dosing regimens. It may also provide insight into inter-individual differences and may aid to identify the most important mechanisms underlying therapy resistance.

Our group previously described an assay for the determination of aza-dCTP concentrations in peripheral blood mononuclear cells (PBMCs) [6]. The present paper will focus on quantitative determination of aza-CTP in PBMCs. In order to develop an accurate assay several obstacles had to be conquered. These issues will be discussed in this paper.

Since aza-CTP was not commercially available, a reference standard had to be synthesized in-house. The small scale synthesis procedure as well as the characterization of the reaction mixture are described. The quantification of the aza-CTP reference standard was complicated by the instability of azacitidine in aqueous solution. 


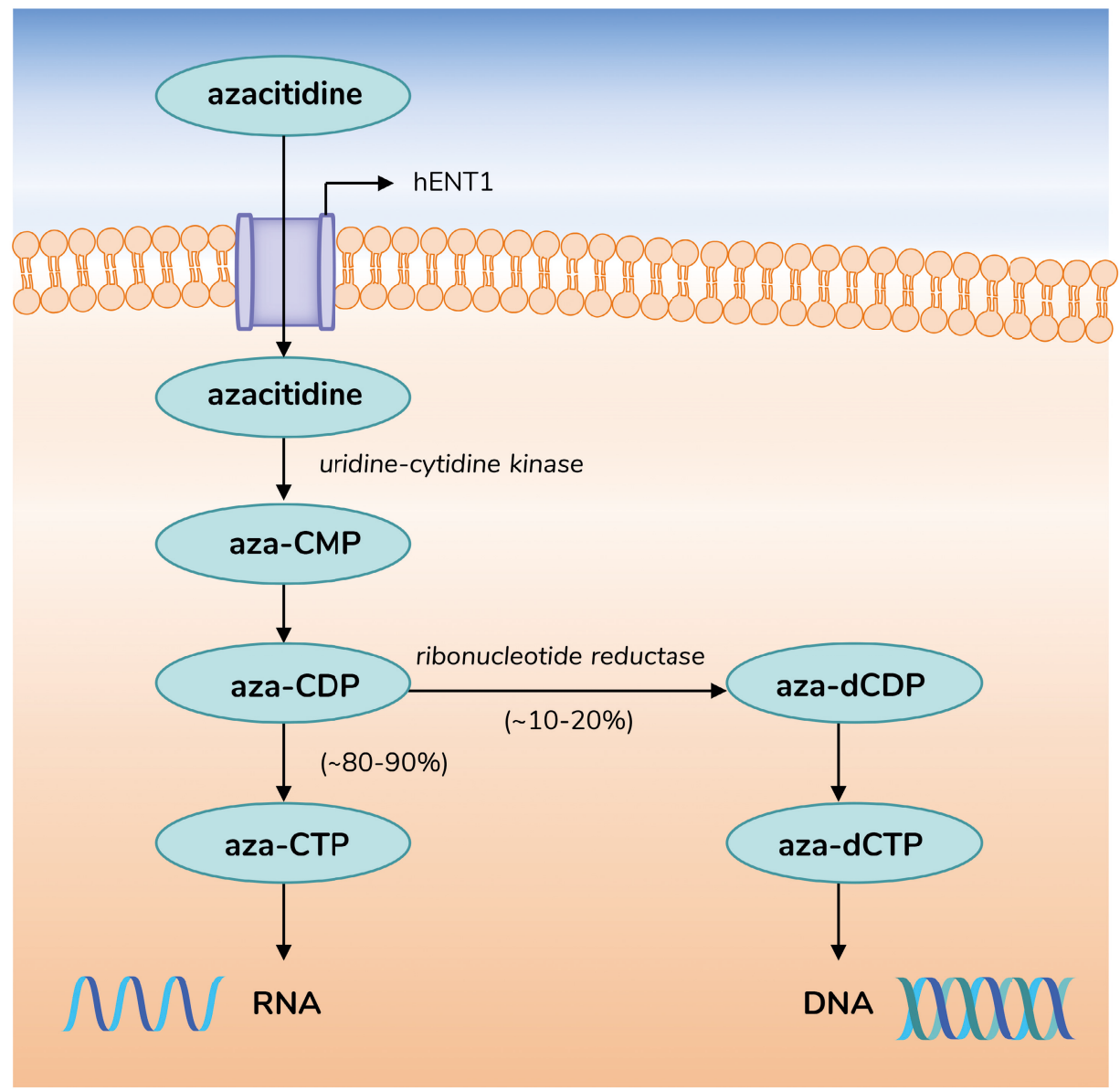

Figure 1. Intracellular activation pathways of azacitidine

Abbreviations: aza-CMP, azacitidine 5'-monophosphate; aza-CDP, azacitidine 5'-diphosphate; aza-CTP, azacitidine 5'-triphosphate; aza-dCDP, 5-aza-2'-deoxycytidine 5'-diphosphate; azadCTP, 5-aza-2'-deoxycytidine 5'-triphosphate; hENT1, human equilibrative nucleoside transporter 1.

Interference by endogenous nucleotides proved to be more complex for azaCTP than it had been for aza-dCTP. The previously published aza-dCTP assay was only hindered by the naturally occurring $\left[{ }^{13} \mathrm{C}\right]$ - and $\left[{ }^{15} \mathrm{~N}\right]$-isotopes of deoxycytidine triphosphate (dCTP). The authors corrected for this interference by measuring the principal isotope of $d C T P$ and calculated the $\left[{ }^{13} \mathrm{C}\right]-$ and $\left[{ }^{15} \mathrm{~N}\right]-$ dCTP interference based on the isotopic distribution [6]. A similar approach was not an option for the determination of aza-CTP, as the interference problem is more extensive here. 


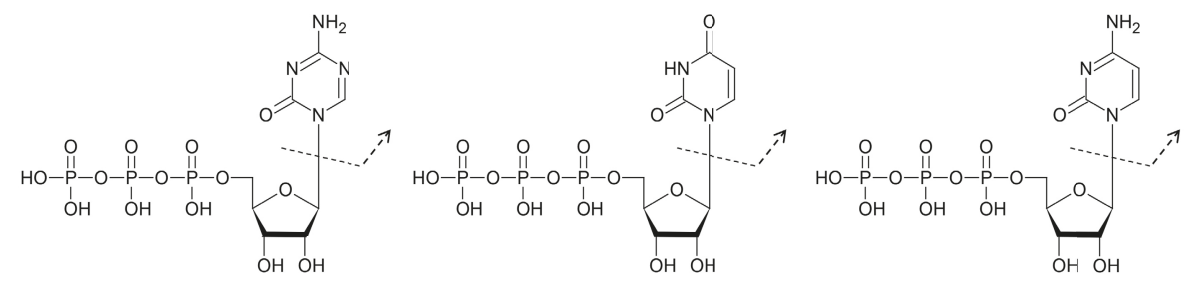

azacitidine 5'-triphosphate

uridine 5'-triphosphate

cytidine 5'-triphosphate

Figure 2. Chemical structures of azacitidine 5'-triphosphate, uridine 5'-triphosphate and cytidine 5'-triphosphate. The arrows indicate the proposed fragmentation patterns.

Table 1. Precursor and product ions of azacitidine 5'-triphosphate, uridine 5 '-triphosphate and the $\left[{ }^{13} \mathrm{C}\right]$ - and $\left[{ }^{15} \mathrm{~N}\right]$-isotopes of cytidine 5 '-triphosphate.

\begin{tabular}{lll}
\hline Compound & Precursor ion $(\mathrm{m} / \mathrm{z})$ & Product ion $(\mathrm{m} / \mathrm{z})$ \\
\hline azacitidine 5'-triphosphate & 484.9876 & 113.0463 \\
uridine 5'-triphosphate & 484.9764 & 113.0351 \\
{$\left[{ }^{13} \mathrm{C}\right]$-isotope of cytidine 5'-triphosphate } & 484.9957 & 113.0544 \\
{$\left[{ }^{15} \mathrm{~N}\right]$-isotope of cytidine 5'-triphosphate } & 484.9894 & 113.0481 \\
\hline
\end{tabular}

The chemical structure of aza-CTP is highly similar to the structures of the endogenous nucleotides cytidine triphosphate (CTP) and uridine triphosphate (UTP) (Fig. 2). These endogenous nucleotides are highly abundant in cells, whereas aza-CTP is only present at low levels. Thus, to quantify aza-CTP in PBMC material without bias, separation from these endogenous nucleotides is required. However, considering the small structural differences at the nucleobase site of the molecule and the influential phosphate groups at the other site, chromatographic separation is difficult. Separation through mass spectrometry is therefore an interesting option. However, the monoisotopic molecular masses of aza-CTP, UTP and the naturally occurring $\left[{ }^{13} \mathrm{C}\right]$ - and $\left[{ }^{15} \mathrm{~N}\right]$-isotopes of CTP differ less than 0.02 Da (Table 1), and efforts to select a specific $\mathrm{MS}^{2}$-fragment for aza-CTP using triple quadrupole mass spectrometry remained without success. Therefore, we investigated whether it was feasible to separate these highly resembling nucleotides based on high-resolution mass spectrometry using a linear trap quadrupole (LTQ) coupled with an Orbitrap. This paper describes this successful approach to quantify aza-CTP in PBMCs. 


\section{MATERIALS AND METHODS}

\subsection{Chemicals}

Azacitidine was kindly provided by Celgene Corporation (Summit, NJ, USA). 1,8-Bis(dimethylamino)naphthalene (Proton sponge), trimethylphosphate (TMP), tributylamine (TBA), N,N-dimethylformamide (DMF) and phosphoric acid crystals were purchased from Sigma-Aldrich (St. Louis, MO, USA). Phosphorous oxychloride $\left(\mathrm{POCl}_{3}\right), 1 \mathrm{M}$ triethylammonium bicarbonate $\mathrm{pH} 8.5$ and $3 \AA$ molecular sieves were from Fluka (St. Louis, MO, USA). All solvents used in the synthesis were purchased as anhydrous and required no further drying.

HPLC supra-gradient-grade methanol and acetonitrile were obtained from Biosolve Ltd (Valkenswaard, The Netherlands). Lichrosolve water for chromatography, ammonium acetate, glacial acetic acid and ammonia solution 25\% all were from Merck (Darmstadt, Germany).

The Ficoll-Paque plus density gradient medium used for the isolation of PBMCs was purchased from GE Healthcare. Phosphate buffered saline (PBS) was from Sigma-Aldrich, and tetrahydrouridine from Calbiochem (La Jolla, CA, USA).

\subsection{Synthesis of aza-CTP}

Since azacitidine 5'-triphosphate (aza-CTP) was not commercially available, a reference standard was synthesized in-house by nucleoside phosphorylation with phosphorous oxychloride $\left(\mathrm{POCl}_{3}\right)$. The development and optimization of this small scale synthesis procedure were extensively described [7] and the procedure was previously applied to synthesize 5-aza-2'-deoxycytidine 5'-triphosphate (aza-dCTP) [6].

About $50 \mu \mathrm{mol}$ azacitidine was dissolved in $2.5 \mathrm{~mL}$ trimethylphosphate. Proton sponge $(100 \mu \mathrm{mol})$ was added and the mixture was cooled on ice. A large excess (32 equivalents) of $\mathrm{POCl}_{3}$ was added dropwise under continuous stirring over a period of 5 hours. Then an excess (20 equivalents) of tributylammonium phosphate (250 mM solution in N,N-dimethylformamide) was added under 
continuous stirring. After 2 hours, the reaction was quenched by pouring it into $15 \mathrm{~mL}$ cold $1 \mathrm{M}$ triethylammonium bicarbonate. The reaction product was lyophilized, dissolved in water and stored at $-70^{\circ} \mathrm{C}$.

\subsubsection{Characterization of the reaction mixture}

The reaction mixture was characterized by liquid chromatography with tandem mass spectrometry detection. The LC-MS/MS system used was previously described for the qualitative analysis of 5-aza-2'-deoxycytidine nucleotides [6]. In brief, a sample volume of $25 \mu \mathrm{L}$ was injected onto a porous graphitic carbon column (Hypercarb, $100 \times 2.1 \mathrm{~mm}$ ID, $5 \mu \mathrm{m}$ particles; Thermo Scientific (part of Thermo Fisher Scientific), Waltham, MA, USA). Separation was achieved using a gradient of $1 \mathrm{mM}$ ammonium acetate in acetonitrile-water (15:85, $\mathrm{v} / \mathrm{v}) \mathrm{pH} 5$ and $25 \mathrm{mM}$ ammonium bicarbonate in acetonitrile-water (15:85, $\mathrm{v} / \mathrm{v}$ ), at a flow of $0.25 \mathrm{~mL} / \mathrm{min}$. Detection was performed using an API4000 triple quadrupole mass spectrometer (AB Sciex, Framingham, MA, USA). The compound dependent scanning conditions were optimized for the azacitidine nucleotides and are summarized in Table 2.

\subsubsection{Quantification of aza-CTP in the reaction mixture}

To determine the aza-CTP concentration in the reaction mixture, the porous graphitic carbon LC-MS/MS system, as described above, was extended with an UV detector (Agilent technologies Inc., Santa Clara, CA, USA). The MS/MS data were used for identification of the relevant peaks. The actual quantification was based on the UV absorption at $242 \mathrm{~nm}$ using azacitidine as a reference standard, based on the assumption that the nucleoside and the nucleotides have identical molar absorptions.

For determination of the aza-CTP concentration, the azacitidine synthesis mixture was diluted 100 times with water. Azacitidine reference standards $(10 \mu \mathrm{g} / \mathrm{mL})$ were freshly prepared in duplicate. All samples were prepared on ice and directly placed in the autosampler at $4{ }^{\circ} \mathrm{C}$ before analysis. 
Table 2. Settings for the API4000 triple quadrupole mass spectrometer for the characterization of the reaction mixture and quantification of aza-CTP in this mixture.

\begin{tabular}{llllll}
\hline Compound & $\begin{array}{l}\text { Precursor } \\
\text { ion } \\
(\mathrm{m} / \mathbf{z})\end{array}$ & $\begin{array}{l}\text { Product ion } \\
(\mathrm{m} / \mathbf{z})\end{array}$ & $\begin{array}{l}\text { Declustering } \\
\text { potential } \\
(\mathrm{V})\end{array}$ & $\begin{array}{l}\text { Collision } \\
\text { energy } \\
(\mathrm{V})\end{array}$ & $\begin{array}{l}\text { Collision cell } \\
\text { exit potential } \\
(\mathrm{V})\end{array}$ \\
\hline azacitidine & 245.2 & 113.2 & 50 & 25 & 10 \\
aza-CMP & 325.1 & 113.2 & 50 & 25 & 10 \\
aza-CDP & 405.1 & 113.2 & 50 & 30 & 10 \\
aza-CTP & 485.1 & 113.2 & 65 & 40 & 7 \\
\hline
\end{tabular}

\subsection{Development of a bio-analytical assay}

\subsubsection{LC-LTQ-Orbitrap system}

Liquid chromatography-high-resolution mass spectrometry analyses were performed on a Dionex Ultimate 3000 series liquid chromatography system consisting of a LPG-3400M pump and a WPS-3000(RS) autosampler (Dionex (part of Thermo Fisher Scientific), Sunnyvale, CA, USA) coupled to an LTQOrbitrap Discovery mass spectrometer (Thermo Scientific (part of Thermo Fisher Scientific), Waltham, MA, USA).

The autosampler was thermostatted at $4{ }^{\circ} \mathrm{C}$ and a sample volume of $25 \mu \mathrm{L}$ was loaded onto a Biobasic AX column $(50 \mathrm{~mm} \times 2.1 \mathrm{~mm}$ ID, particle size 5 $\mu \mathrm{m}$ ) preceded by a $10 \mathrm{~mm}$ drop-in guard cartridge (both Thermo Scientific) maintained at ambient temperatures. Mobile phase $A$ consisted of a mixture of $10 \mathrm{mM} \mathrm{NH}_{4} \mathrm{Ac}$ in $\mathrm{ACN} / \mathrm{H}_{2} \mathrm{O}(30: 70, \mathrm{v} / \mathrm{v})$ adjusted to $\mathrm{pH} 6.0$, and mobile phase $B$ consisted of $1 \mathrm{mM} \mathrm{NH}{ }_{4} \mathrm{Ac}$ in $\mathrm{ACN} / \mathrm{H}_{2} \mathrm{O}(30: 70, \mathrm{v} / \mathrm{v})$ adjusted to $\mathrm{pH} 10.5$. The eluent $\mathrm{pH}$ was increased and the $\mathrm{NH}_{4} \mathrm{Ac}$ concentration was decreased in mobile phase $B$ relative to mobile phase $A$ to obtain a good separation of the monophosphates, diphosphates and triphosphates as previously described [8,9]. The mobile phases were delivered at a flow of $0.25 \mathrm{~mL} / \mathrm{min}$ with the following stepwise gradient: 0-0.5 min: $10 \%$ B; 0.51-1.75 min: $50 \%$ B; 1.76 7.50 min: $100 \%$ B; $7.60-9.50$ min: $10 \%$ B with an increased flow rate of 0.50 $\mathrm{mL} / \mathrm{min}$; 9.50-10.0 min: $10 \% \mathrm{~B}$ with the initial flow rate. A switching valve was used to direct the flow to waste during the first 3 minutes and after 7 minutes. The total run time was 10 minutes. 
The LTQ-Orbitrap mass spectrometer was equipped with an electrospray ionization probe and operated in the positive ion mode. Source settings and ion optics were optimized through continuous infusion and flow injection analysis (FIA). The ionspray voltage was kept at $8 \mathrm{kV}$, with a capillary temperature of $275^{\circ} \mathrm{C}$. The capillary voltage was set at $20 \mathrm{~V}$ and the tube lens at $110 \mathrm{~V}$. The sheath gas, auxiliary gas and sweep gas flow rate were set at 40, 20 and 0 (arbitrary units), respectively. Optimization of the ion optics resulted in the following settings: multipole 00 offset $-2 \mathrm{~V}$, lens 0 voltage $-3.67 \mathrm{~V}$, multipole 0 offset $-5.50 \mathrm{~V}$, lens 1 voltage $-12 \mathrm{~V}$, gate lens voltage $-61 \mathrm{~V}$, multipole 1 offset $-22 \mathrm{~V}$, multipole RF amplitude $770 \mathrm{~V}$, and front lens $-6 \mathrm{~V}$. $\mathrm{MS}^{2}$ scans were performed for parent mass $m / z 485$ with scan range $m / z$ 105-114. The highest signal for the fragment with $\mathrm{m} / \mathrm{z} 113$ was obtained using collision induced dissociation (CID), with a normalized collision energy of 15 , an activation $Q$ of 0.17 , and activation time of $30 \mathrm{~ms}$. The isolation width was $1.0(\mathrm{~m} / \mathrm{z})$. Fragment ions were detected in the Orbitrap at a resolution of 30,000 (full width at half maximum at $\mathrm{m} / \mathrm{z} 400$ ). Data were acquired in both the profile and the centroid mode using Xcalibur (version 2.0) software (Thermo Scientific (part of Thermo Fisher Scientific), Sunnyvale, CA, USA).

\subsubsection{Sample preparation}

Clinical samples were obtained by drawing $16 \mathrm{~mL}$ whole blood in lithium heparin tubes. Immediately after collection a tetrahydrouridine solution was added to a final concentration of $100 \mu \mathrm{g} / \mathrm{mL}$ to prevent ex vivo deamination of azacitidine $[6,10]$. PBMCs were isolated as previously described [11]. In brief, the blood samples were centrifuged, after which the buffy coat was resuspended in phosphate buffered saline (PBS) and layered over Ficoll paque plus density gradient. After centrifugation the PBMCs were collected and washed with PBS. The thus obtained cell pellet was resuspended in $70 \mu \mathrm{L}$ PBS. Resulting in a homogeneous cell suspension with a total volume of approximately 100 $\mu \mathrm{L}$. A $30 \mu \mathrm{L}$ aliquot of this cell suspension was taken for a cell count using a hematology analyzer (Cell-Dyn Sapphire; Abbott Diagnostics, Abbott Park, IL, USA), and a $60 \mu \mathrm{L}$-aliquot of the cell suspension was used for determination of the aza-CTP concentration. For the latter purpose the cells were lysed by the addition of $100 \mu \mathrm{L}$ methanol followed by extensive vortex mixing for 1 minute. 
Then the sample was centrifuged at $3000 \times \mathrm{g}$, for 5 minutes at $4^{\circ} \mathrm{C}$, after which the supernatant (cell lysate) was collected and stored at $-70^{\circ} \mathrm{C}$ until analysis.

For the preparation of blanks, blank human leukocyte buffy coat (Sanquin, Amsterdam, the Netherlands) from $500 \mathrm{~mL}$ whole blood was diluted to 500 $\mathrm{mL}$ with PBS and further treated as whole blood. The obtained blank PBMC suspension was diluted to $42 \times 10^{6} \mathrm{PBMCs} / \mathrm{mL}$ with PBS before lysis. The thus obtained blank lysate suspension was centrifuged for 5 minutes at $3000 \times \mathrm{g}$, after which the supernatant was stored at $-70{ }^{\circ} \mathrm{C}$.

For the preparation of calibration standards and quality control samples, working solutions were prepared by dilution of the aza-CXP synthesis product in water. A volume of $5 \mu \mathrm{L}$ of each working solution was added to $100 \mu \mathrm{L}$ of blank PBMC Iysate (cell suspension:methanol $=60: 100, \mathrm{v} / \mathrm{v}$ ) to obtain aza-CTP calibration standards in the range from 10.1 to $281 \mathrm{nM}$. In the same manner quality control samples were prepared at four concentration levels to assess the accuracy and precision. The calibration standards were prepared and analyzed in duplicate, the quality control samples in fivefold.

To determine the selectivity of the method, PBMCs were isolated from the blood of six different individuals who did not use azacitidine. The thus obtained blank samples were checked for interferences. In addition, the six batches were spiked with aza-CTP at two concentration levels (50.8 and $225 \mathrm{nM}$ ) to assess the influence of endogenous material on the accuracy of the method.

\subsection{Clinical application of the assay}

The described assay was applied for determination of the aza-CTP concentration in PBMC samples obtained from two patients who were treated with azacitidine. Both patients were diagnosed with myelodysplastic syndrome and were treated with $75 \mathrm{mg} / \mathrm{m}^{2}$ azacitidine subcutaneously for seven days (interrupted in the weekends), every four weeks. For patient 1 (male, 62 years) blood samples were collected at day six of the eighth treatment cycle, 60 and 120 minutes after azacitidine administration. For patient 2 (female, 72 years) blood samples were drawn at day seven of the third treatment cycle just before azacitidine administration (pre-dose) and 30,60 and 90 minutes 
after azacitidine administration. Sampling times were chosen considering the plasma pharmacokinetics of azacitidine. Following a standard dose of $75 \mathrm{mg} / \mathrm{m}^{2}$ subcutaneously, maximum plasma concentration $(750 \pm 403 \mathrm{ng} / \mathrm{mL}$ ) is reached after 30 minutes and the mean elimination half-life is about 41 minutes [12]. Blood samples were immediately placed on ice and were further processed as described in Section 2.3.2. Both patients gave informed consent prior to collection of the samples.

The analytical result, expressed as $\mathrm{nM}$ in PBMC lysate, was multiplied with the lysate sample volume to obtain the absolute aza-CTP amount in a sample. This amount was then divided by the number of cells present in the sample to obtain the aza-CTP amount per million PBMCs.

\section{RESULTS AND DISCUSSION}

\subsection{Characterization of the reaction mixture}

After performing the reaction steps as described under 'synthesis of aza-CTP' a mixture of azacitidine, azacitidine monophosphate, azacitidine diphosphate, and azacitidine triphosphate (aza-CXP) was obtained. The MS/MS chromatogram (Fig. 3A1) showed two peaks for the azacitidine monophosphate mass transition. Although previously described that the use of $\mathrm{POCl}_{3}$ in a trimethylphosphate solution results in the selective phosphorylation of the 5'-hydroxyl group of the nucleoside $[13,14]$, the two peaks might indicate that a part of the molecules underwent phosphorylation of one of the other hydroxyl groups. No further experiments were performed to discover the identity of the two peaks in the mass transition of azacitidine monophosphate. Aza-CTP was present in the reaction mixture and the concentration was determined by LC-UV.

\subsection{Quantification of aza-CTP in the reaction mixture}

The quantification process was thwarted by the instability of azacitidine in aqueous solution. Fig. 4 shows the predominant hydrolytic pathway of azacitidine. Beisler et al. (1978) described the formation of $\mathrm{N}$-(formylamidino)- 
A1.

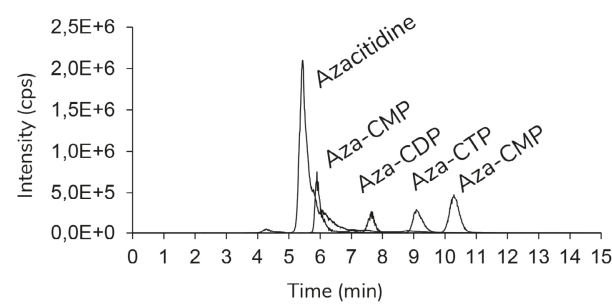

A2.

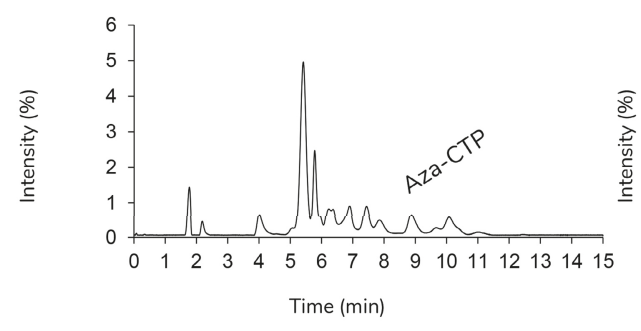

B1.

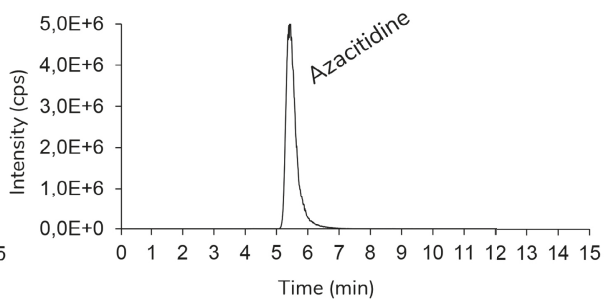

B2.

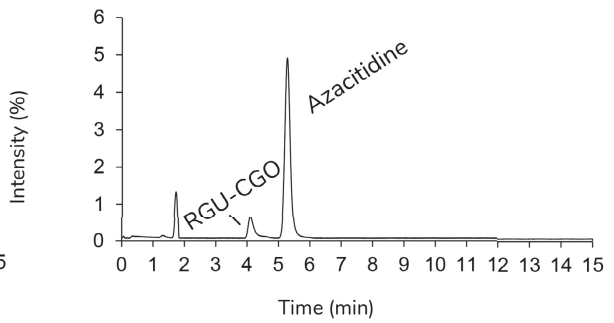

Figure 3. Quantification of aza-CTP in the reaction mixture: chromatograms of the reaction mixture (100 times diluted in water) with MS/MS (A1) and UV (A2) detection, and of azacitidine reference standard $(10 \mu \mathrm{g} / \mathrm{mL})$ with MS/MS (B1) and UV (B2) detection.

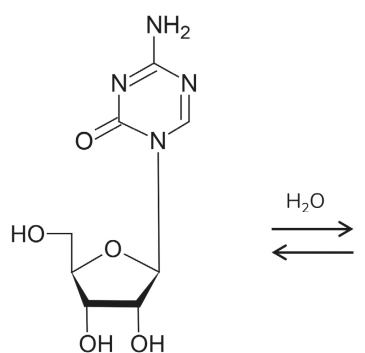

Azacitidine

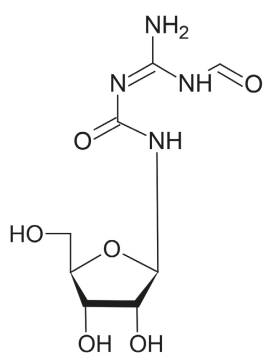

$\mathrm{N}$-(formylamidino)N'- $\beta$-D-ribofuranosylurea (RGU-CHO)<smiles>NC(N)=NC(=O)NC1OC(CO)C(O)C1O</smiles>

1- $\beta$-D-ribofuranosyl-

3-guanylurea (RGU)

Figure 4. Predominant hydrolytic pathway of azacitidine 
$N^{\prime}-\beta$-D-ribofuranosylurea (RGU-CHO) as a consequence of the nucleophilic attack by water on the C- 6 position of azacitidine which was followed by ring opening. $\mathrm{RGU}-\mathrm{CHO}$ readily equilibrates to azacitidine, and is more slowly converted to 1- $\beta$-D-ribofuranosyl-3-guanylurea (RGU) by irreversible loss of the $\mathrm{N}$-formyl group [15].

Fig. 3B2 shows a representative chromatogram of a freshly prepared azacitidine reference solution, which was immediately injected on the HPLC-UV-MS/MS system. Using MS and MS/MS spectra, the peak at 4.12 minutes was identified as RGU-CHO and the peak at 5.37 minutes as azacitidine. In time, the azacitidine peak decreased to the benefit of the RGU-CHO peak: $12 \%$ of azacitidine was lost within 10 hours at $4{ }^{\circ} \mathrm{C}$. This complicated the quantification.

Azacitidine and RGU-CHO do not have the same absorption characteristics. However, after correction based upon the ratio of the extinction coefficients $\left(\varepsilon_{242} \mathrm{RGU}-\mathrm{CHO} / \varepsilon_{242}\right.$ azacitidine $\approx 2.75$ ) [15], the sum of the two peak areas remained constant in time. This 'corrected sum area' [(peak area RGU-CHO / $2.75)$ + peak area azacitidine] was used to quantify aza-CTP in the reaction mixture.

For quantification, the area of the aza-CTP peak detected with UV (Fig. 3A2) was compared with the corrected sum area of the reference standard peaks (Fig. 3B2). The synthesis mixture was found to contain $591 \mu \mathrm{M}$ aza-CTP.

aza- СТP concentration $=$ azacitidine concentration $\times \frac{\text { area aza- CTP peak }}{\text { corrected sum area azacitidine reference peaks }}$

\subsection{Development of a bio-analytical assay}

\subsubsection{Separation from endogenous nucleotides}

Question was whether the LTQ-Orbitrap was able to differentiate between aza-CTP and the endogenous nucleotides UTP, $\left[{ }^{15} \mathrm{~N}\right]-\mathrm{CTP}$ and $\left[{ }^{13} \mathrm{C}\right]-\mathrm{CTP}$ based on the accurate masses. The product ion mass spectrum of $\mathrm{m} / \mathrm{z} 485$ showed three peaks in blank PBMC lysate originating from UTP, $\left[{ }^{15} \mathrm{~N}\right]-\mathrm{CTP}$ and $\left[{ }^{13} \mathrm{C}\right]-$ 
A.

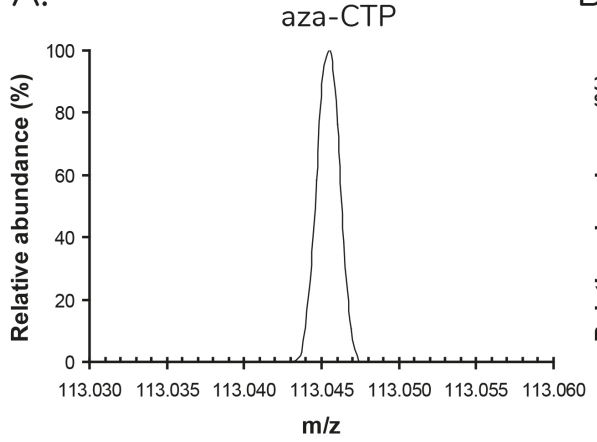

C.

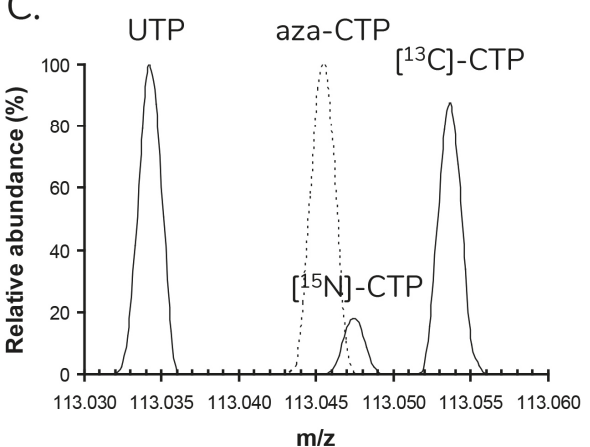

B.

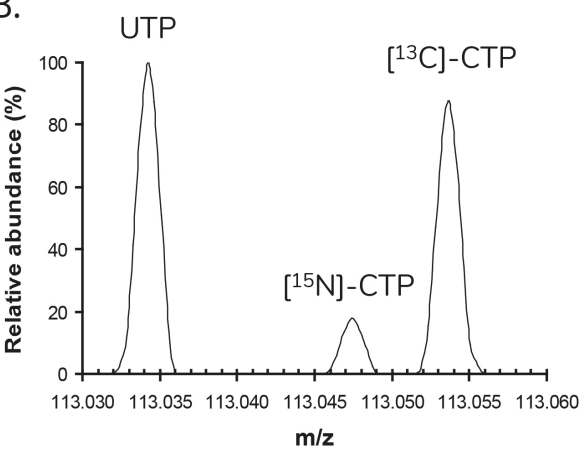

Figure 5. Accurate LTQ-Orbitrap mass measurements: the product ion mass spectra of $\mathrm{m} / \mathrm{z} 485$ (with a scan range of $\mathrm{m} / \mathrm{z} 105-114$ ) from 3.90 to $4.15 \mathrm{~min}$ for aza-CTP (A), blank PBMC lysate (B), and an overlay of both (C).

CTP (Fig. 5B). The overlay plot of this product ion mass spectrum and the mass spectrum obtained from an aqueous aza-CTP solution (Fig. $5 \mathrm{C}$ ) demonstrates that aza-CTP was clearly separated from the UTP and $\left[{ }^{13} \mathrm{C}\right]-C T P$ product ions. However the $\left[{ }^{15} \mathrm{~N}\right]-\mathrm{CTP}$ product ion was not complete baseline separated from the aza-CTP product ion.

These results were consistent with the theoretical expectation based on the mass differences and the resolving power of the Orbitrap instrument. For nearbaseline separation of two adjacent masses, the difference between the two masses should be at least twice of the full width at half maximum (FWHM) [16]. Calculation of the FWHM (with the assumption that the resolving power was 30,000 at $\mathrm{m} / \mathrm{z}$ 113) showed that the mass differences between aza-CTP, UTP 
and $\left[{ }^{13} \mathrm{C}\right]$-CTP were indeed larger than twice the FWHM, whereas the mass difference between aza-CTP and $\left[{ }^{15} \mathrm{~N}\right]-\mathrm{CTP}$ was not.

The Orbitrap acquires product ion spectra from $\mathrm{m} / \mathrm{z} 485$ across a mass range of $m / z 105$ to 114 . To process the data, extracted ion chromatograms for azaCTP (m/z 113.0430-113.0470) were obtained. The accurate mass range of the aza-CTP product ion was determined experimentally by injection of an aqueous aza-CTP solution on the LC-LTQ-Orbitrap system. At the retention time of the peak in the total ion current (TIC) chromatogram, a mass spectrum was obtained in profile mode (Fig. 5A). From this mass spectrum the accurate (i.e. measured) mass of the aza-CTP product ion was established ( $\mathrm{m} / \mathrm{z} 113.0430-113.0470)$.

Extracted ion chromatograms (XICs) were obtained post data acquisition by selecting the accurate transition out of the MS-MS/MS data. Fig. 6 shows the TICs as well as the XICs for blank PBMC lysate and PBMC lysate spiked with aza-CTP at LLOQ level. This figure clearly illustrates that the TICs cannot be used for quantification as a result of the endogenous interferences, whereas the accurate mass XICs potentially can. For all of the six blank PBMC batches, the XIC was free from interferences at the retention time of aza-CTP.

\subsubsection{Quantification of aza-CTP in PBMC lysate}

Calibration curves were constructed based on the peak areas in the accurate mass XICs using linear regression with a $1 / x^{2}$ weighting factor, where $x$ is the concentration. The best results were obtained using the centroid mode for data acquisition. The linearity was evaluated by means of back-calculated concentrations of the calibration standards. A calibration standard was rejected if the deviation from the nominal concentration exceeded $\pm 20 \%$. No more than one third of the calibration standards were allowed to be rejected from the calibration curves. The assay was linear over the concentration range from 40.7 to $281 \mathrm{nM}$ (in cell lysate) with a correlation coefficient $\left(r^{2}\right)$ of 0.9596 . The deviations of the mean back-calculated concentrations from nominal were between -8.3 and $8.9 \%$ with coefficient of variation values $\leq 22.0 \%$. One calibration standard was rejected as the deviation from the nominal concentration exceeded $\pm 20 \%$. 

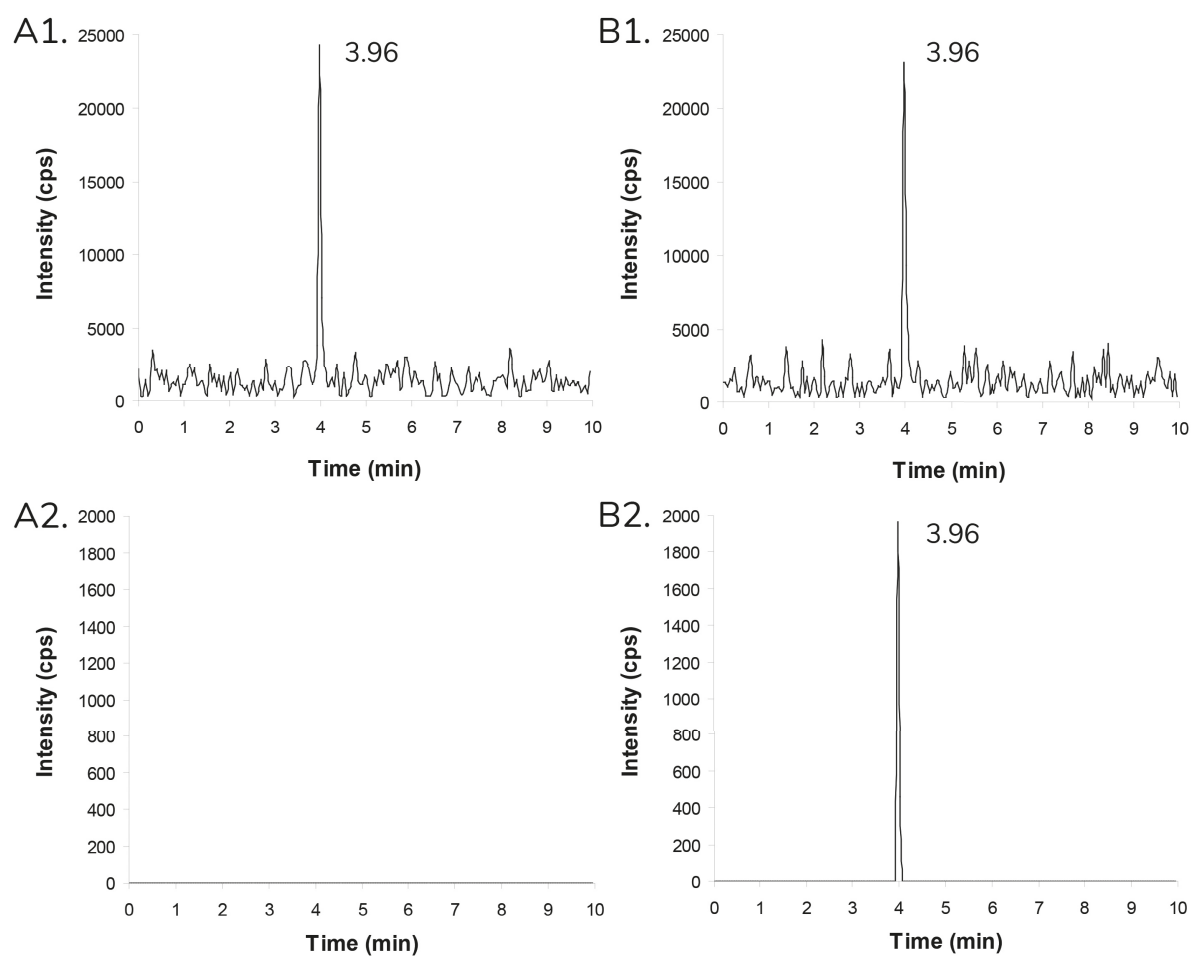

Figure 6. Accurate LTQ-Orbitrap mass measurements: the total ion current chromatograms (1) and extracted ion chromatograms (2) for blank PBMC lysate (A) and PBMC lysate spiked with $40.7 \mathrm{nM}$ aza-CTP (B).

Quality control samples at four levels were analyzed in fivefold in order to assess the accuracy and precision of the assay. The differences between the nominal and the determined concentration were used to calculate the intraassay accuracy and were between -1.1 and $9.5 \%$ deviation at all concentration levels. The coefficients of variation were calculated to assess the intra-assay precision of the method and were all $\leq 13.2 \%$. For the samples processed in six different batches of PBMC lysate, at least 4 out of 6 samples were within $\pm 20 \%$ deviation at both concentration levels.

To examine the matrix effect, the peak areas of PBMC lysate spiked with azaCTP were compared with the peak areas of a methanol/PBS mixture (in the same proportion as for cell lysis, 100:60, v/v) spiked with aza-CTP. The matrix factor was determined in triplicate at four concentrations and was found to be 
constant for the various aza-CTP concentrations. The mean matrix factor was 0.322 with a $C V$ value of $14.1 \%$.

Carry over was assessed by injecting a blank sample after the highest calibration standard. As there was no signal in the XIC of the blank sample, carry over was found to be acceptable.

\subsection{Clinical application of the assay}

The aza-CTP concentrations determined in patient samples are presented in Fig. 7. This shows that the assay can be applied for measurement of the intracellular aza-CTP concentrations in patient samples at least up to 120 minutes after administration. To our knowledge, this is the first time that azaCTP concentrations were measured in cell material from patients treated with azacitidine.

As mentioned, our group previously described an assay for the determination of 5-aza-2'-deoxycytidine triphosphate (aza-dCTP) concentrations in PBMC samples obtained from patients who were treated with decitabine [6]. The azaCTP concentrations presented in the current paper are 2.5 to 55 times higher than the published aza-dCTP concentrations. The difference between the concentrations found reflects the common difference between ribonucleotide and deoxyribonucleotide concentrations. Endogenous ribonucleotide contents are several orders of magnitudes higher than the corresponding deoxyribonucleotide contents $[17,18]$. 


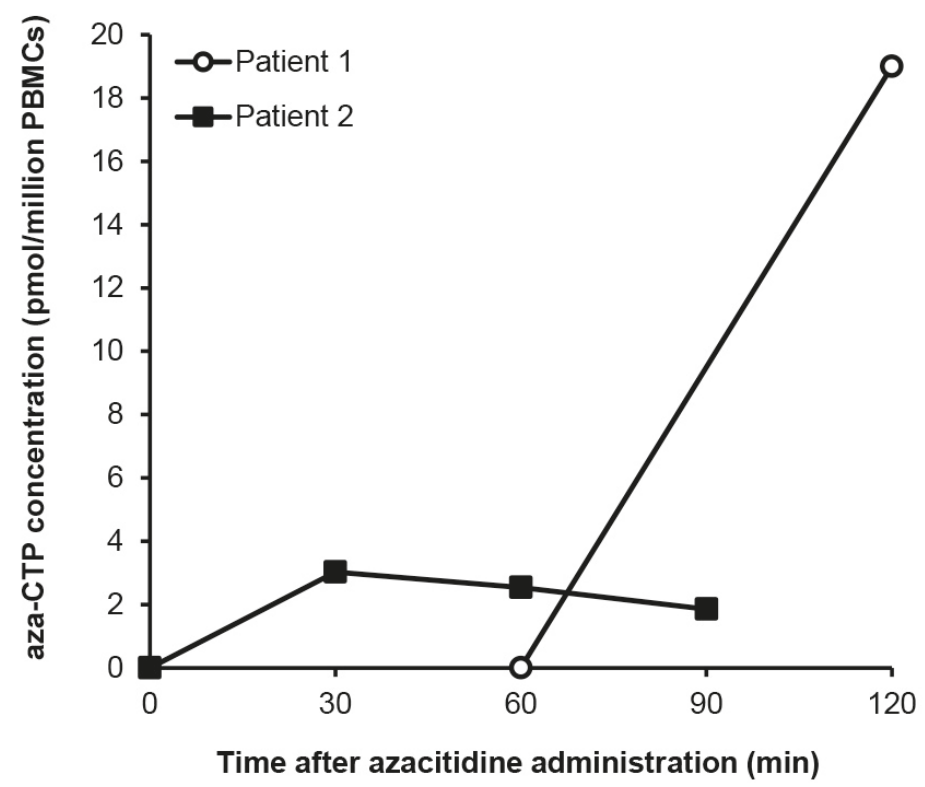

Figure 7. Aza-CTP concentration per million PBMCs measured in patient samples after the subcutaneous administration of $75 \mathrm{mg} / \mathrm{m}^{2}$ azacitidine.

\section{CONCLUSIONS}

This is the first reported assay for determination of the aza-CTP concentration in PBMCs. This highly selective assay has the ability to separate aza-CTP from the endogenous nucleotides UTP and the $\left[{ }^{13} \mathrm{C}\right]$-isotope of CTP based on their accurate masses. Although the resolution was not sufficient to differentiate between aza-CTP and the $\left[{ }^{15} \mathrm{~N}\right]$-isotope of CTP, the contribution of the latter was negligible and did not bias the quantification of aza-CTP. The assay is useful to determine aza-CTP concentrations in PBMC lysate within the range from 40.7 to $281 \mathrm{nM}$. Assuming that an average cell suspension extracted from $16 \mathrm{~mL}$ whole blood contains 10 to 42 million PBMCs per $\mathrm{mL}$, this range corresponds with 2.58/10.9-17.8/74.9 pmol aza-CTP per million PBMCs. We conclude that the present method can be applied for determination of the azaCTP concentration in PBMC samples obtained from patients who were treated with azacitidine. 


\section{REFERENCES}

[1] E.J.B. Derissen, J.H. Beijnen, J.H.M. Schellens, Concise drug review: azacitidine and decitabine, Oncologist 18 (2013) 619-624.

[2] L.H. Li, E.J. Olin, H.H. Buskirk, L.M. Reineke, Cytotoxicity and mode of action of 5 -azacytidine on L1210 leukemia, Cancer Res. 30 (1970) 2760-2769.

[3] C. Stresemann, F. Lyko, Modes of action of the DNA methyltransferase inhibitors azacytidine and decitabine, Int. J. Cancer 123 (2008) 8-13.

[4] J.K. Christman, 5-Azacytidine and 5-aza2'-deoxycytidine as inhibitors of DNA methylation: mechanistic studies and their implications for cancer therapy, Oncogene 21 (2002) 5483-5495.

[5] A. Quintás-Cardama, F.P. Santos, G. GarciaManero, Therapy with azanucleosides for myelodysplastic syndromes, Nat. Rev. Clin. Oncol. 7 (2010) 433-444.

[6] R.S. Jansen, H. Rosing, P.W. Wijermans, R.J. Keizer, J.H.M. Schellens, J.H. Beijnen, Decitabine triphosphate levels in peripheral blood mononuclear cells from patients receiving prolonged low-dose decitabine administration: a pilot study, Cancer Chemother. Pharmacol. 69 (2012) 1457-1466.

[7] R.S. Jansen, H. Rosing, J.H.M. Schellens, J.H. Beijnen, Facile small scale synthesis of nucleoside 5'-phosphate mixtures, Nucleosides Nucleotides Nucleic Acids 29 (2010) 14-26.

[8] R.S. Jansen, H. Rosing, C.J. de Wolf, J.H. Beijnen, Development and validation of an assay for the quantitative determination of cladribine nucleotides in MDCKII cells and culture medium using weak anion-exchange liquid chromatography coupled with tandem mass spectrometry, Rapid Commun. Mass Spectrom. 21 (2007) 4049-4059.

[9] R.S. Jansen, H. Rosing, W. Kromdijk, R. ter Heine, J.H.M. Schellens, J.H. Beijnen, Simultaneous quantification of emtricitabine and tenofovir nucleotides in peripheral blood mononuclear cells using weak anionexchange liquid chromatography coupled with tandem mass spectrometry, J. Chromatogr. B: Anal. Technol. Biomed. Life Sci. 878 (2010) 621-627.
[10] M. Zhao, M.A. Rudek, P. He, C. Hartke, S. Gore, M.A. Carducci, S.D. Baker, Quantification of 5 -azacytidine in plasma by electrospray tandem mass spectrometry coupled with high-performance liquid chromatography, J. Chromatogr. B: Anal. Technol. Biomed. Life Sci. 813 (2004) 81-88.

[11] S.A. Veltkamp, M.J. Hillebrand, H. Rosing, R.S. Jansen, E.R. Wickremsinhe, E.J. Perkins, J.H.M. Schellens, J.H. Beijnen, Quantitative analysis of gemcitabine triphosphate in human peripheral blood mononuclear cells using weak anion-exchange liquid chromatography coupled with tandem mass spectrometry, J. Mass Spectrom. 41 (2006) 1633-1642.

[12] G. Marcucci, L. Silverman, M. Eller, L. Lintz, C.L Beach, Bioavailability of azacitidine subcutaneous versus intravenous in patients with the myelodysplastic syndromes, J. Clin. Pharmacol. 45 (2005) 597-602.

[13] M. Yoshikawa, T. Kato, T. Takenishi, A novel method for phosphorylation of nucleosides to 5'-nucleotides, Tetrahedron Lett. 50 (1967) 5065-5068.

[14] M. Yoshikawa, T. Kato, T. Takenishi, Studies of phosphorylation. III. Selective phosphorylation of unprotected nucleosides, Bull. Chem. Soc. Jpn. 42 (1969) 3505-3508.

[15] J.A. Beisler, Isolation, characterization, and properties of a labile hydrolysis product of the antitumor nucleoside, 5-azacytidine, J. Med. Chem. 21 (1978) 204-208.

[16] J.L. Josephs, HRMS: current usage, future directions and the promise of integration with unified data streams suited to post-acquisition mining, Bioanalysis 4 (2012) 471-476.

[17] P. Chen, Z. Liu, S. Liu, Z. Xie, J. Aimiuwu, J. Pang, R. Klisovic, W. Blum, M.R. Grever, G. Marcucci, K.K. Chan, A LC-MS/MS method for the analysis of intracellular nucleoside triphosphate levels, Pharm. Res. 26 (2009) 1504-1515.

[18] S. Cohen, M. Megherbi, L.P. Jordheim, I. Lefebvre, C. Perigaud, C. Dumontet, J. Guitton, Simultaneous analysis of eight nucleoside triphosphates in cell lines by liquid chromatography coupled with tandem mass spectrometry, J. Chromatogr. B: Anal. Technol. Biomed. Life Sci. 877 (2009) 3831-3840. 


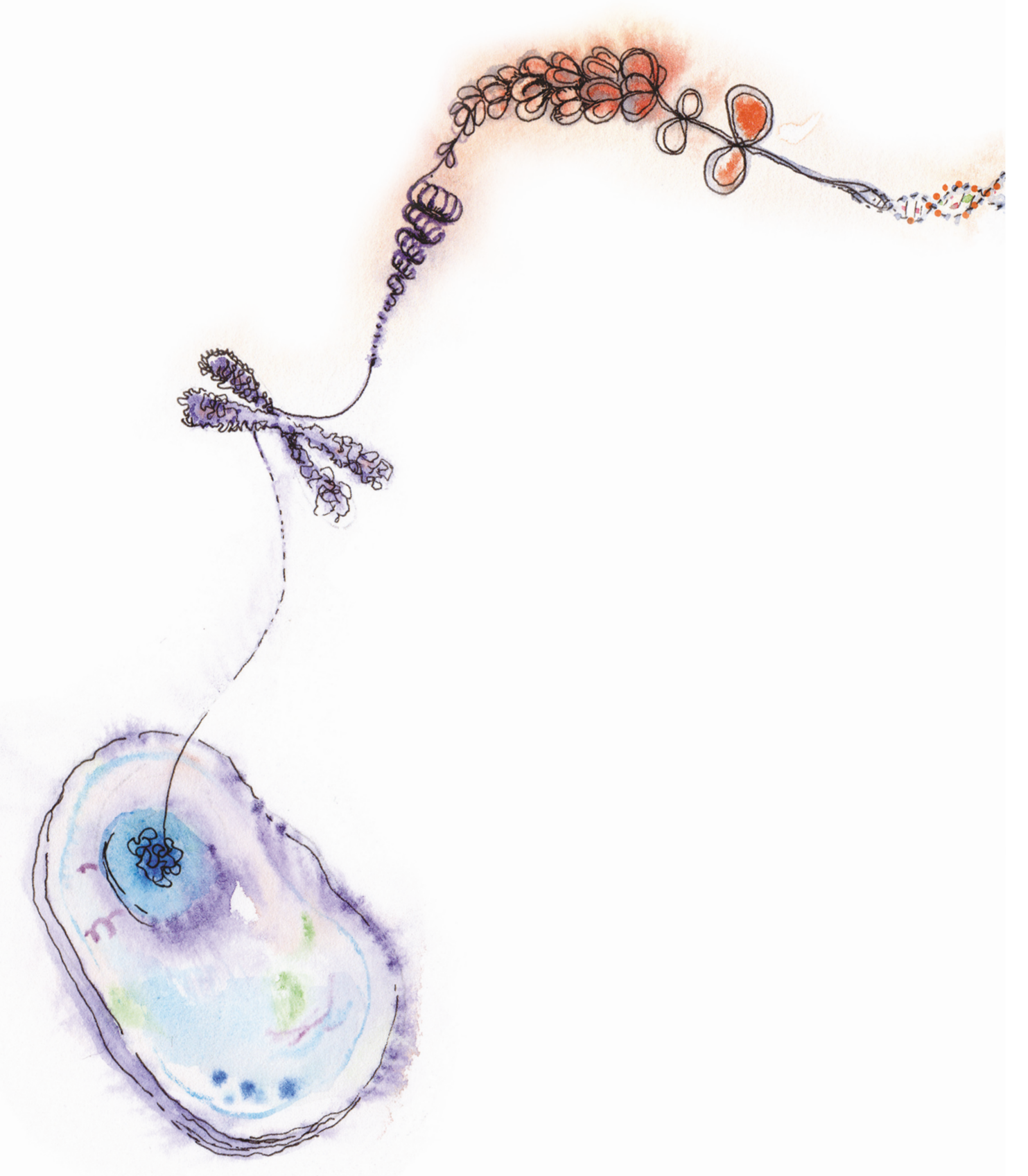




\section{5-Fluorouracil and Capecitabine}

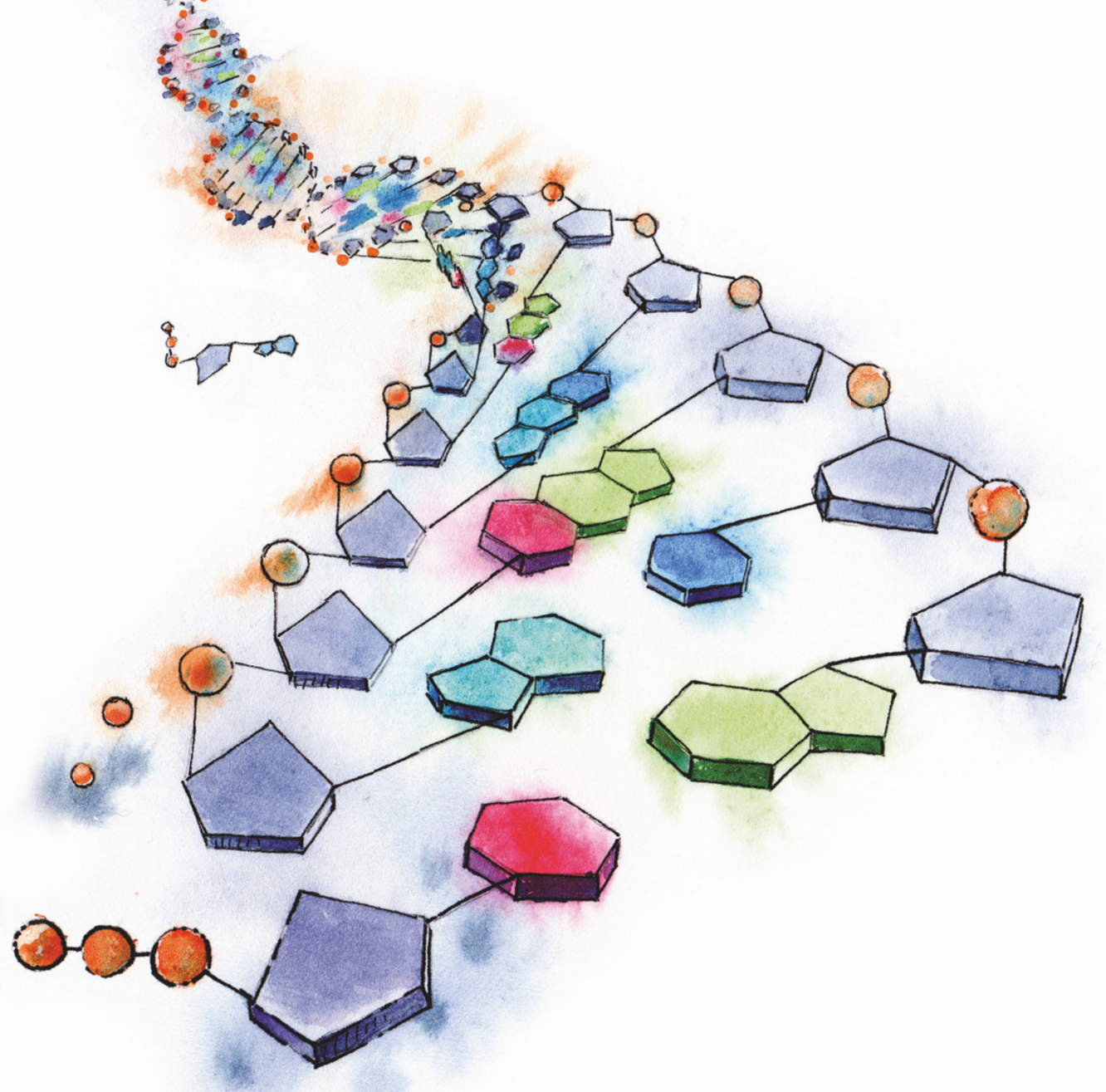




\section{CHAPTER 3.1}

\section{Development of an LC-MS/MS assay for the quantitative determination of the intracellular 5-fluorouracil nucleotides responsible for the anticancer effect of 5-fluorouracil}

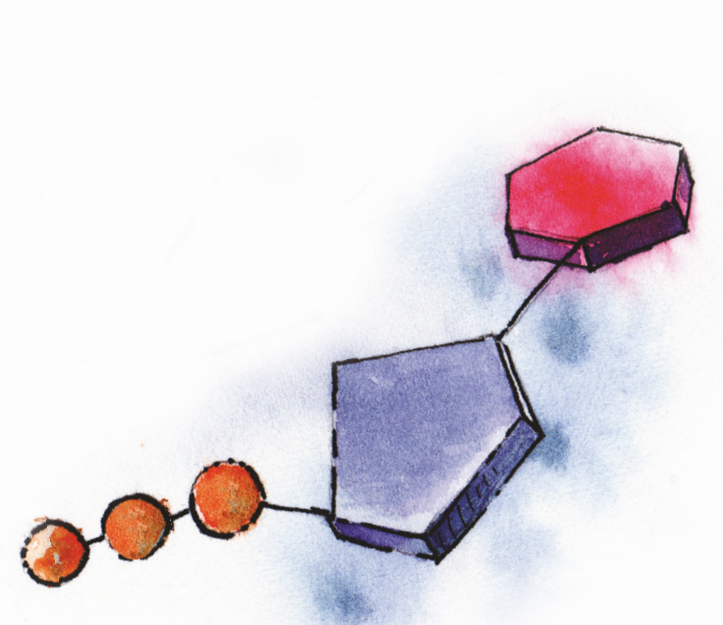

Ellen J.B. Derissen

Michel J.X. Hillebrand Hilde Rosing Jan H.M. Schellens Jos H. Beijnen 


\section{ABSTRACT}

5-Fluorouracil (5-FU) and its oral prodrug capecitabine are among the most widely used chemotherapeutics. For cytotoxic activity, 5-FU requires cellular uptake and intracellular metabolic activation. Three intracellular formed metabolites are responsible for the antineoplastic effect of 5-FU: 5-fluorouridine 5'-triphosphate (FUTP), 5-fluoro-2'-deoxyuridine 5'-triphosphate (FdUTP) and 5-fluoro-2'-deoxyuridine 5'-monophosphate (FdUMP).

In this paper, we describe the development of an LC-MS/MS assay for quantification of these active 5-FU nucleotides in peripheral blood mononuclear cells (PBMCs). Because the intracellular 5-FU nucleotide concentrations were very low, maximization of the release from the cell matrix and minimization of interference were critical factors. Therefore, a series of experiments was performed to select the best method for cell lysis and nucleotide extraction. Chromatography was optimized to obtain separation from endogenous nucleotides, and the effect of different cell numbers was examined.

The assay was validated for the following concentration ranges in PBMC lysate: 0.488-19.9 nM for FUTP, 1.66-67.7 nM for FdUTP and 0.748-30.7 nM for FdUMP. Accuracies were between -2.2 and $7.0 \%$ deviation for all analytes, and the coefficient of variation values were $\leq 4.9 \%$.

The assay was successfully applied to quantify 5-FU nucleotides in PBMC samples from patients treated with capecitabine and patients receiving 5-FU intravenously. FUTP amounts up to $3054 \mathrm{fmol} / 10^{6}$ PBMCs and FdUMP levels up to $169 \mathrm{fmol} / 10^{6}$ PBMCs were measured. The FdUTP concentrations were below the lower limit of quantification. To our knowledge, this is the first time that 5-FU nucleotides were quantified in cells from patients treated with 5-FU or capecitabine without using a radiolabel.

Keywords: 5-fluorouracil; 5-fluorouridine triphosphate (FUTP); 5-fluorodeoxyuridine triphosphate (FdUTP); 5-fluorodeoxyuridine monophosphate (FdUMP); LC-MS/MS 


\section{INTRODUCTION}

The anticancer drug 5-fluorouracil (5-FU) has been in clinical use for more than 50 years and is still one of the most widely used chemotherapeutics. Together with its oral prodrug capecitabine, 5-FU has remained the mainstay in the treatment of a range of cancer types, including those originating in the gastrointestinal tract, breast and head and neck region.

5-FU is a uracil analog with a fluorine atom at the C-5 position in place of a hydrogen. For cytotoxic activity, 5-FU requires cellular uptake and metabolic activation by cellular phosphorylases and kinases (Fig. 1). Based on experiments with radiolabeled 5-FU, three of the intracellular formed metabolites (nucleotides) are responsible for the antineoplastic effect of 5-FU [1]. These metabolites are 5-fluorouridine 5'-triphosphate (FUTP), 5-fluoro2'-deoxyuridine 5'-triphosphate (FdUTP) and 5-fluoro-2'-deoxyuridine 5'-monophosphate (FdUMP; Fig. 2). In brief, FUTP is incorporated into RNA and interferes with normal RNA processing and function. FdUTP is incorporated into DNA, leading to pathological DNA structures and ultimately cell death. FdUMP inhibits thymidylate synthase, the enzyme that catalyzes the transformation of deoxyuridine monophosphate (dUMP) to deoxythymidine monophosphate (dTMP). Inhibition of thymidylate synthase by FdUMP leads to accumulation of dUTP and depletion of dTTP. This imbalance has deleterious consequences for DNA synthesis and repair, ultimately leading to cell death (Fig. 1) [2,3].

It would be useful to quantify the three intracellularly formed nucleotides that are responsible for the antineoplastic effect of $5-\mathrm{FU}$, in cells of patients that are treated with 5-FU or capecitabine. This would provide insight into the amount of 'activated drug' that has reached the site of action, information that could ultimately lead to more substantiated dosage regimens. In addition, the measurement of the intracellular nucleotide concentrations could provide insight into the mechanisms underlying treatment resistance. Given these benefits, it is not surprising that for other nucleoside analogs, measurements of intracellular monophosphates, diphosphates and triphosphates are increasingly being applied [4-8]. For 5-FU, such measurements would also be useful. 


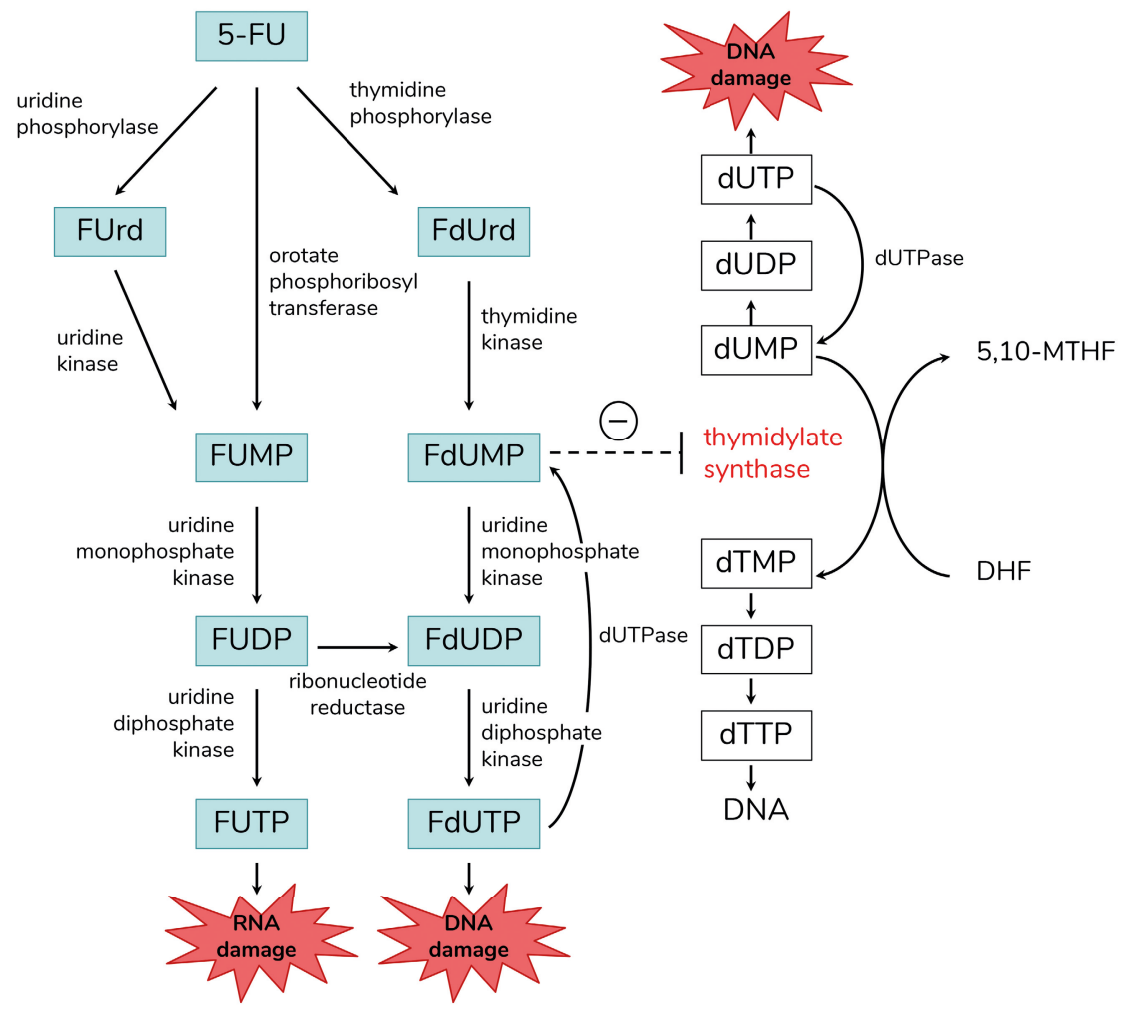

Figure 1. Intracellular metabolism and mechanisms of action of 5-fluorouracil

This figure shows only the anabolic route, which gives rise to the active metabolites. The catabolic route, which inactivates 5-FU and leads to elimination of the drug, is omitted.

Abbreviations: 5-FU, 5-fluorouracil; FUrd, 5-fluorouridine; FdUrd, 5-fluoro-2'-deoxyuridine; FUMP, 5-fluorouridine 5'-monophosphate; FUDP, 5-fluorouridine 5'-diphosphate; FUTP, 5-fluorouridine 5'-triphosphate; FdUMP, 5-fluoro-2'-deoxyuridine 5'-monophosphate; FdUDP, 5-fluoro-2'-deoxyuridine 5'-diphosphate; FdUTP, 5-fluoro-2'-deoxyuridine 5'-triphosphate; dUMP, 2'-deoxyurdine 5'-monophosphate; dUDP, 2'-deoxyurdine 5'-diphosphate; dUTP, 2'-deoxyurdine 5'-triphosphate; dTMP, 2'-deoxythymidine 5'-monophosphate; dTDP, 2'-deoxythymidine 5'-diphosphate; dTTP, 2'-deoxythymidine 5'-triphosphate; DHF, dihydrofolate; 5,10-MTHF, 5,10-methylene-tetrahydrofolate.

In the past decades, various analytical methods for the quantification of 5-FU nucleotides were described. However, most assays are not suitable for the measurement of cell samples obtained from patients that are treated with 5-FU or capecitabine. Some assays lack sensitivity, such as the methods reported for the quantification of FdUMP by HPLC-UV [9] or using capillary electrophoresis with UV-detection [10]. Also fluorine-19 nuclear magnetic resonance ( $\left.{ }^{19} \mathrm{~F}-\mathrm{NMR}\right)$ 
spectroscopy was not sufficiently sensitive for quantification of the individual 5-FU nucleotides in patients samples [11]. In addition, a substantial portion of the reported assays utilized a radioactive label $[1,12]$, which makes these assays also useless for the measurement of samples of patients that are treated with unlabeled drug.

For a long time, competitive-binding assays, wherein quantification was based on the displacement of radiolabeled isotopes, were the only option to measure FdUMP in patient samples [13-15]. However, these assays were quite laborious and not practical for clinical application. In 2009 Carli et al. described an LC-MS/ MS assay for the quantification of FdUMP in cultured cell models [16]. This assay was much easier to apply and, if it is sensitive enough, it might also be used for the determination of FdUMP concentrations in patients samples.

To our knowledge, no LC-MS/MS assays have been reported for the quantification of FdUTP and FUTP. Therefore, our aim was to develop an LC-MS/MS assay for simultaneous quantification of the three 5-FU nucleotides that are responsible for the antineoplastic effect of 5-FU in peripheral blood mononuclear cells (PBMCs). PBMCs were used to reflect the intracellular metabolic activation because these cells can be easily obtained after administration of 5-FU or capecitabine.

In general, the development of an adequate assay for the determination of intracellular nucleotide concentrations remains a challenge. The main obstacles are: 1 .) the very low concentrations present in the cells of patients; 2 .) the extent of release from the cell matrix, which is difficult to assess; and 3.) the presence of endogenous nucleotides, which are highly similar and may cause interference.

For the 5-FU nucleotides, the development of an appropriate assay proved even more difficult than for other nucleotide analogs because intracellular 5-FU nucleotide concentrations, especially those following capecitabine treatment, are much lower $[17,18]$. Maximization of the release from the cell matrix and minimization of endogenous interference were therefore critical factors in obtaining an appropriate assay. This paper describes how the obstacles mentioned above were overcome to develop a selective and sensitive assay for 
the quantification of the three nucleotides that are responsible for the anticancer effect of 5-FU.

\section{MATERIALS AND METHODS}

\subsection{Chemicals}

FUTP and FdUTP were purchased from Sierra Bioresearch (Tucson, AZ, USA). Because isotopically labeled FUTP and FdUTP were not commercially available, ${ }^{13} \mathrm{C}_{9}{ }^{15} \mathrm{~N}_{2}$-labeled uridine 5'-triphosphate (*UTP, Fig. 2) was tested as a potential internal standard. This *UTP was obtained from Sigma-Aldrich (St. Louis, MO, USA), just like uridine, 5-fluorouridine and 5-fluoro-2'-deoxyuridine. UPLCMS grade methanol, acetonitrile and water were obtained from Biosolve Ltd (Valkenswaard, The Netherlands). Ammonium acetate (purity 98\%), glacial acetic acid, $25 \%$ ammonia solution, $50 \%$ sodium hydroxide solution and potassium dihydrogen phosphate (purity $>99.5 \%$ ) were purchased from Merck (Darmstadt, Germany). Tetrabutylammonium dihydrogen phosphate (purity >99\%) was obtained from AppliChem (Darmstadt, Germany). The Ficoll-Paque plus density gradient medium used for the isolation of PBMCs was purchased from GE Healthcare. Phosphate buffered saline pH 7.4 (PBS) was from SigmaAldrich.

\subsection{Preparation and quantification of the reference and internal standard solutions}

Because only the nucleoside triphosphates were commercially available, the nucleoside monophosphates and diphosphates were obtained in-house by thermal degradation. To this end, separate aqueous solutions of FUTP, FdUTP and *UTP (as potential internal standard) were placed in a water bath at 90 ${ }^{\circ} \mathrm{C}$. In this paper the suffix $-\mathrm{XP}$ is used to refer to the obtained mixtures of nucleoside monophosphates, diphosphates and triphosphates.

Thermal degradation of the nucleoside triphosphates into lower phosphates was monitored using the HPLC-UV system previously described for the analysis 


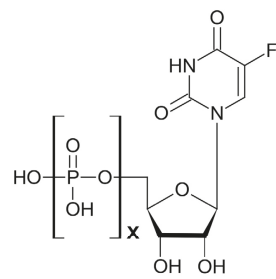

FUXP

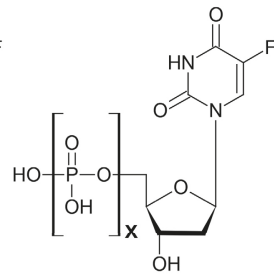

FdUXP

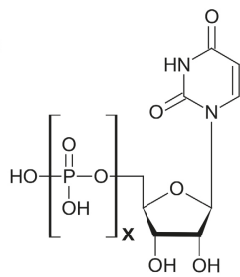

UXP

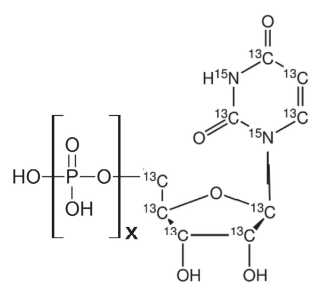

*UXP

Figure 2. Chemical structures of 5-fluorouridine-X-phosphate, 5-fluoro-2'-deoxyuridine-X-phosphate, uridine-X-phosphate and ${ }^{13} \mathrm{C}_{9}{ }^{15} \mathrm{~N}_{2}$-labeled uridine-X-phosphate (tested as internal standard). $X$ may be mono, di or tri.

of emtricitabine and tenofovir with some minor adjustments [19]. The mobile phase consisted of $10 \mathrm{mM}$ tetrabutylammonium dihydrogen phosphate with $70 \mathrm{mM}$ potassium dihydrogen phosphate in water adjusted to $\mathrm{pH} 7$ and $7 \%$ methanol $(\mathrm{v} / \mathrm{v})$, and it was delivered isocratically to a Synergi hydro-RP column (150 mm × $2.0 \mathrm{~mm}$ ID, 4 um particles; Phenomenex, Torrance, CA, USA) with a flow rate of $0.25 \mathrm{~mL} / \mathrm{min}$. A volume of $10 \mu \mathrm{L}$ was injected using the autosampler thermostated at $4{ }^{\circ} \mathrm{C}$. Absorption was measured at $268 \mathrm{~nm}$ for FUXP and FdUXP and at $262 \mathrm{~nm}$ for *UXP.

For each solution, thermal degradation was continued until approximately equal amounts of nucleoside monophosphate, diphosphate and triphosphate were obtained. This was achieved after approximately $4 \mathrm{~h}$ at $90^{\circ} \mathrm{C}$. After the samples were thoroughly cooled on ice, the nucleotide concentrations of the FUXP, FdUXP and *UXP mixtures were determined using the above HPLC-UV system. 5-fluorouridine, 5-fluoro-2'-deoxyuridine and uridine were used as calibration standards based on the assumption that the nucleoside and the nucleotides have identical molar absorptions. To this end, three 5-fluorouridine, 5-fluoro2'-deoxyuridine and uridine reference solutions were prepared from separate weighings. The peak areas obtained by injecting these reference solutions in duplicate were compared to those obtained by injecting the nucleotide solutions with unknown concentrations in triplicate. The FUXP and FdUXP mixtures were combined and diluted in water to obtain working solutions. The *UXP solution was diluted in water to obtain the internal standard working solution. All solutions were stored at $-70^{\circ} \mathrm{C}$. 


\subsection{Sample preparation}

Clinical samples were obtained by drawing $16 \mathrm{~mL}$ whole blood in lithium heparin tubes. Samples were processed immediately after blood collection, and processing was executed on ice. After centrifugation (10 min at $1500 \times \mathrm{g}$ at 4 ${ }^{\circ} \mathrm{C}$ ), the buffy coat was collected and resuspended in cold phosphate buffered saline $\mathrm{pH} 7.4$ (PBS) to a total volume of $6 \mathrm{~mL}$. This diluted buffy coat was then carefully layered over $4 \mathrm{~mL}$ cold Ficoll-Paque plus density gradient, avoiding mixing of the layers. After centrifugation $\left(20 \mathrm{~min}\right.$ at $550 \times \mathrm{g}$ at $4{ }^{\circ} \mathrm{C}$ without brake), a two-layer system was formed. The PBMCs, forming a white interface between the upper and lower layers, were collected and washed with $35 \mathrm{~mL}$ cold PBS. After centrifugation ( $5 \mathrm{~min}$ at $1500 \times \mathrm{g}$ at $4^{\circ} \mathrm{C}$ ), the supernatant was thoroughly removed. The cell pellet was resuspended in $70 \mu \mathrm{L}$ PBS, resulting in a homogeneous cell suspension with a total volume of approximately 100 $\mu \mathrm{L}$. A $30 \mu \mathrm{L}$ aliquot of this cell suspension was 10 times diluted with PBS and then used to perform a cell count using a hematology analyzer (Cell-Dyn Sapphire; Abbott Diagnostics, Abbott Park, IL, USA). A $60 \mu \mathrm{L}$ aliquot of the cell suspension was used for determination of the 5-FU nucleotide concentrations. To this end, cells were lysed by the addition of $100 \mu \mathrm{L}$ methanol, followed by extensive vortex mixing for $1 \mathrm{~min}$. After centrifugation ( $5 \mathrm{~min}$ at $3000 \times \mathrm{g}$ at $4{ }^{\circ} \mathrm{C}$ ), the supernatant (PBMC lysate) was collected and stored at $-70^{\circ} \mathrm{C}$ until analysis.

Directly prior to the LC-MS/MS analysis, after vortex mixing, a $100 \mu \mathrm{L}$ sample was processed by the addition of $5 \mu \mathrm{L}$ internal standard working solution. Then, the samples were again vortex mixed, centrifuged $\left(5 \mathrm{~min}\right.$ at $3000 \times \mathrm{g}$ at $\left.4^{\circ} \mathrm{C}\right)$ and transferred to autosampler vials.

\subsection{Preparation of CALs and QCs}

For the preparation of blanks, a blank human leukocyte buffy coat from 500 $\mathrm{mL}$ whole blood (Sanquin, Amsterdam, The Netherlands) was diluted to 500 $\mathrm{mL}$ with PBS. This diluted buffy coat was layered over cold Ficoll-Paque plus density gradient and further treated as described in Section 2.3. The thus obtained blank PBMC suspension was diluted with PBS to $42 \times 10^{6}$ PBMCs/ $\mathrm{mL}$. This was previously reported as the average cell concentration after processing $15 \mathrm{~mL}$ whole blood [20]. Blank PBMC suspensions were lysed by 
the addition of methanol, wherein the volume ratio of 60:100 was maintained. After centrifugation ( $5 \mathrm{~min}$ at $3000 \times \mathrm{g}$ at $4^{\circ} \mathrm{C}$ ), the supernatant (blank PBMC lysate) was stored at $-70{ }^{\circ} \mathrm{C}$.

Directly prior to the LC-MS/MS analysis, six non-zero calibration standards (CALs) with FUTP concentrations of 0.488 , 0.974, 1.53, 1.99, 9.74 and 19.9 nM, FdUTP concentrations of 1.66, 3.31, 5.22, 6.77, 33.1 and $67.7 \mathrm{nM}$, and FdUMP concentrations of $0.748,1.50,2.36,3.07,15.0$ and $30.7 \mathrm{nM}$ were prepared by adding $5 \mu \mathrm{L}$ of the appropriate working solution to $95 \mu \mathrm{L}$ blank PBMC lysate. In the same manner, quality control samples (QCs) were prepared at mid-level (4.98 nM for FUTP, $16.9 \mathrm{nM}$ for FdUTP and $7.66 \mathrm{nM}$ for FdUMP). The CALs and QCs were further processed as described in Section 2.3.

\subsection{LC-MS/MS system}

The LC-MS/MS analyses were performed on an Acquity ultra performance liquid chromatography (UPLC) system (Waters Corporation, Milford, MA, USA) coupled to a QTrap 5500 mass spectrometer (AB Sciex, Framingham, MA, USA). Samples were kept at $4{ }^{\circ} \mathrm{C}$ in the autosampler tray, and a sample volume of 10 $\mu \mathrm{L}$ was loaded onto a Biobasic AX column (50 mm $\times 2.1 \mathrm{~mm}$ ID, particle size 5 $\mu \mathrm{m}$ ) preceded by a $10 \mathrm{~mm}$ drop-in guard cartridge (both Thermo Scientific). The column oven was maintained at $40^{\circ} \mathrm{C}$. Mobile phase $A$ consisted of a mixture of $10 \mathrm{mMNH}_{4} \mathrm{Ac}$ in $\mathrm{ACN} / \mathrm{H}_{2} \mathrm{O}(30: 70, \mathrm{v} / \mathrm{v})$ adjusted to $\mathrm{pH} 6.0$, and mobile phase $B$ consisted of $1 \mathrm{mM} \mathrm{NH}_{4} \mathrm{Ac}$ in $\mathrm{ACN} / \mathrm{H}_{2} \mathrm{O}(30: 70, \mathrm{v} / \mathrm{v})$ adjusted to $\mathrm{pH} 10.5$. The eluent $\mathrm{pH}$ was increased, and the $\mathrm{NH}_{4} \mathrm{Ac}$ concentration was decreased in mobile phase $B$ relative to mobile phase $A$ to obtain a good separation of the nucleoside monophosphates, diphosphates and triphosphates. The mobile phases were delivered at a flow rate of $0.25 \mathrm{~mL} / \mathrm{min}$ with the following stepwise gradient: initially $10 \% \mathrm{~B}$; 0.1-1.6 min: $60 \% \mathrm{~B} ; 1.65-2.1 \mathrm{~min}: 80 \% \mathrm{~B} ; 2.11-5.0$ min: $100 \%$ B; 5.1-6.9 min: $10 \%$ B with an increased flow rate of $0.50 \mathrm{~mL} / \mathrm{min}$; after which the flow returned to the initial conditions. The total run time was 7 min. A switching valve was used to direct the flow to waste during the first 1.5 $\min$ and after $6.9 \mathrm{~min}$ of the run.

The QTrap 5500 mass spectrometer was equipped with an electrospray ionization probe and was operated in the negative ion mode. The source 
settings and compound-dependent scan conditions were optimized through continuous infusion and flow injection analysis (FIA). The ionspray voltage was kept at $-4500 \mathrm{~V}$ at a temperature of $700{ }^{\circ} \mathrm{C}$. The curtain gas $\left(\mathrm{N}_{2}\right)$ and gas 1 and 2 (zero air) were set at 40 arbitrary units, and the collision-activated dissociation gas $\left(\mathrm{N}_{2}\right)$ was set at 11 arbitrary units. The entrance potential was $-10 \mathrm{~V}$. Scans of $50 \mathrm{~ms}$ were performed for each compound at unit resolution. After optimization, several mass transitions were compared for each analyte, and the mass transitions that resulted in the largest signal to noise ratios were selected (Table 1). Data were acquired and processed using Analyst (version 1.5.2) software (AB Sciex).

Table 1. Selected mass transitions and scan conditions.

\begin{tabular}{llllll}
\hline Compound & $\begin{array}{l}\text { Parent } \\
\text { ion } \\
(\mathrm{m} / \mathrm{z})\end{array}$ & $\begin{array}{l}\text { Product } \\
\text { ion } \\
(\mathrm{m} / \mathrm{z})\end{array}$ & $\begin{array}{l}\text { Declustering } \\
\text { potential } \\
(\mathrm{V})\end{array}$ & $\begin{array}{l}\text { Collision } \\
\text { energy } \\
(\mathrm{V})\end{array}$ & $\begin{array}{l}\text { Collision cell } \\
\text { exit potential } \\
(\mathrm{V})\end{array}$ \\
\hline FUTP & 500.9 & 158.9 & -95 & -42 & -13 \\
\hline FdUTP & 484.8 & 256.8 & -145 & -44 & -21 \\
\hline FdUDP & 404.8 & 275.0 & -105 & -28 & -9 \\
\hline FdUMP & 325.0 & 128.9 & -85 & -26 & -11 \\
\hline *UTP & 493.8 & 158.9 & -165 & -44 & -23 \\
\hline *UMP & 334.1 & 117.2 & -120 & -34 & -9 \\
\hline
\end{tabular}

\section{RESULTS AND DISCUSSION}

\subsection{Development of the assay}

\subsubsection{Maximization of nucleotide release from the cell}

Accurate determination of intracellular nucleotide concentrations requires an efficient extraction from cells. However, evaluation of the extraction recovery is not simple. The intracellular situation cannot be simulated in a spiking experiment because it would not properly reflect cell lysis and extraction from the cell interior. To still be able to select the most suitable method for PBMC Iysis and nucleotide extraction, we performed an experiment with a compound that is naturally present within cells: uridine 5'-triphosphate (UTP). Because the 
chemical structure of UTP is very similar to that of FUTP (Fig. 2), the UTP yields were considered a useful representative for FUTP liberation from the cells.

To compare extraction methods, $60 \mu \mathrm{L}$ PBMC aliquots originating from a single buffy coat were used. Because these aliquots originated from the same buffy coat, their nominal UTP content was presumed to be constant. This provided the means to compare the relative UTP recoveries obtained with different extraction methods. Based on the methods for PBMC lysis and nucleotide extraction found in the literature, different approaches were evaluated. Each method was executed five times. Four extraction solvents were tested: methanol, ethanol, acetonitrile and $1.35 \mathrm{M}$ perchloric acid $\left(\mathrm{HClO}_{4}\right)$. Three different amounts of extraction solvent were compared: 60,100 and $140 \mu \mathrm{L}$. Except for perchloric acid, for which only a volume of $60 \mu \mathrm{L}$ was tested because the perchloric acid treatment required further dilution for neutralization prior to LC-MS/MS analysis (by the addition of $25 \mu \mathrm{L} 1 \mathrm{M}$ ammonium acetate and $10 \mu \mathrm{L} 10 \%$ ammonia). Finally, three different extraction procedures were evaluated. Procedure 1: Addition of extraction solvent to the PBMC suspension, followed by extensive vortex mixing and direct centrifugation, after which the supernatant was collected and stored at $-70^{\circ} \mathrm{C}$. Procedure 2: Addition of extraction solvent to the PBMC suspension, followed by extensive vortex mixing, after which the entire sample (still containing cell debris) was stored at $-70^{\circ} \mathrm{C}$. Thus, centrifugation was suspended until after thawing. Procedure 3: The PBMC suspension was directly stored (as a cell pellet) at $-70^{\circ} \mathrm{C}$. Just prior to LC-MS/MS analysis, the sample was thawed, and extraction solvent was added, followed by extensive vortex mixing, centrifugation and collection of the supernatant. Evaporation of the extraction solvent to concentrate the extracts was not possible due to the instability of the analytes.

The UTP extraction potential of the extraction methods was evaluated using the described LC-MS/MS method. To this end, the method was extended with a mass transition for UTP ( $\mathrm{m} / \mathrm{z} 483 \rightarrow \mathrm{m} / \mathrm{z}$ 159). In addition to a different UTP recovery, the various extraction methods could also cause a different matrix effect by the extraction of diverse other compounds from the cell matrix. However, because this experiment was intended to optimize the sensitivity of the 5-FU nucleotides assay presented in this paper, we were interested in the extraction method with the most appropriate resultant of both matrix effect and 


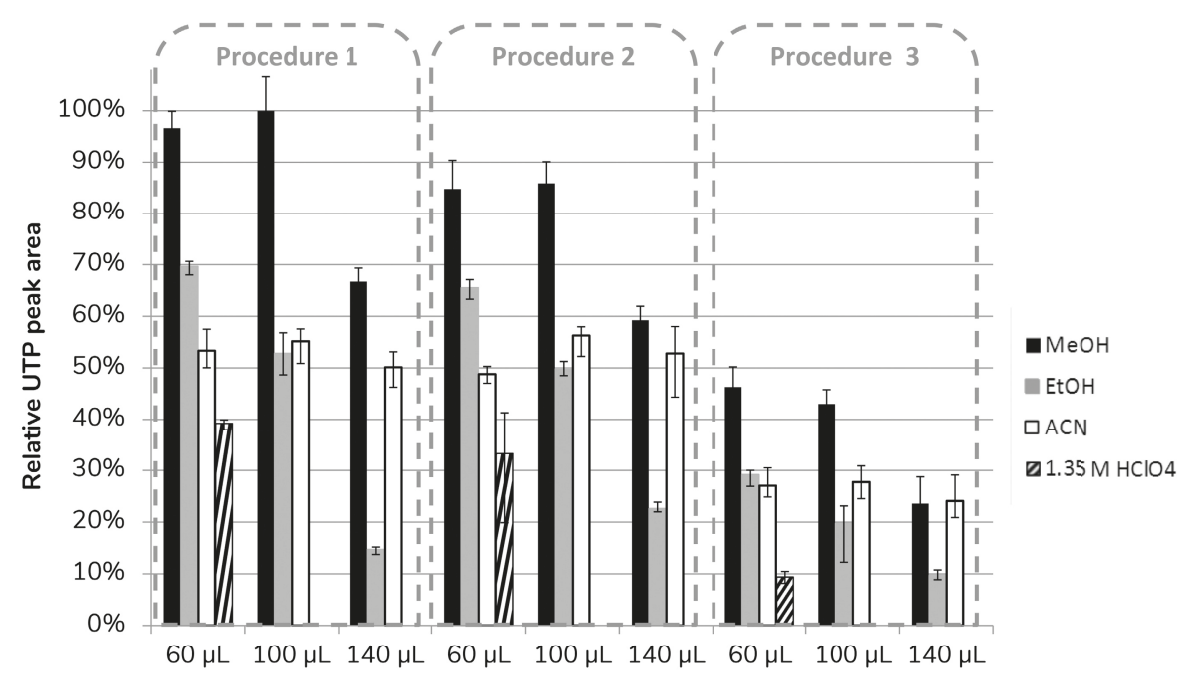

Volume extraction solvent $(\mu \mathrm{L})$

Figure 3. Relative UTP peak areas obtained with different extraction methods. The largest peak area (defined as 100\%) was obtained after extraction with $100 \mu \mathrm{L}$ methanol following procedure 1. Each method was executed five times. The error bars represent the range between the highest and lowest observation.

recovery. Therefore, a pragmatic approach was followed: we searched for the extraction method that resulted in the highest UTP peak areas when analyzing the final extract.

The relative UTP peak areas obtained with the different extraction procedures are depicted in Fig. 3. The largest UTP peak areas were observed after extraction with $100 \mu \mathrm{L}$ methanol according to procedure 1 . The smallest UTP peak areas were obtained after extraction procedure 3 . This extraction procedure was included in the comparison because direct freezing of the cell pellet would cause cell lysis, as PBMCs are not resistant to freezing. However, considering the poor results, this approach is probably detrimental with regard to the stability of UTP, which might be explained by the fact that enzymes were not inactivated by an extraction solvent before storage at $-70^{\circ} \mathrm{C}$.

Because extraction procedure 1 with $100 \mu \mathrm{L}$ methanol gave the most favorable combination of matrix effect and recovery, this extraction procedure was selected for the 5-FU nucleotides assay. Maximization of the nucleotide release 
from the cell was important to obtain maximum assay sensitivity. Furthermore, reproducibility of the recovery is important to obtain adequate assay precision. After extracting five blank PBMC samples with $100 \mu \mathrm{L}$ methanol according to procedure 1 , the coefficient of variation for the UTP peak areas was $6.5 \%$, which was considered acceptable.

\subsubsection{Minimization of endogenous interference}

The obtained LC-MS/MS chromatograms show several endogenous interferences (Fig. 4). In the FUTP and FdUTP transitions, for instance, a peak was detected with a retention time of $3.02 \mathrm{~min}$. Separation from this endogenous peak is essential to quantify FUTP and FdUTP and was achieved by the $80 \%$ eluent $\mathrm{B}$ step in the gradient. The $\mathrm{pH}$ of the eluents is critical to maintain separation. After $24 \mathrm{~h}$, the $\mathrm{pH}$ of eluent $\mathrm{B}$ changed, and the separation deteriorated. Therefore, eluents were prepared freshly before each run. The small peaks observed in the 5-fluoro-2'-deoxyuridine 5'-diphosphate (FdUDP) and FdUMP transitions at $3.20 \mathrm{~min}$ are probably the consequence of in-source degradation of FdUTP. Therefore, chromatographic separation of the monophosphates, diphosphates and triphosphates is a necessity to prevent biased quantitation of the analytes. To examine if this chromatographic separation was achieved, the FdUDP mass transition was also monitored, even if FdUDP was not active.

\subsubsection{Considerations regarding matrix effects and the use of internal standards}

Matrix effects are relevant in LC-MS/MS because co-eluting compounds can interfere during the ionization process. The number of PBMCs varies per sample and may influence the matrix effect [21], so the matrix effect was studied for different cell concentrations. Clinical samples derived from $16 \mathrm{~mL}$ whole blood using the described isolation method were found to contain 10-200 $\times 10^{6}$ PBMCs/mL. Therefore cell suspensions containing 5, 10, 25, 50, 100, 150, 200, 250, 300 and $350 \times 10^{6}$ PBMCs/mL were prepared in PBS using PBMCs freshly isolated from a single donor buffy coat. Sixty microliter aliquots of these cell suspensions were lysed with $100 \mu \mathrm{L}$ methanol. After centrifugation, the PBMC lysate was used to prepare QCs at mid-level (FUTP 4.98 nM, FdUTP 16.9 nM 
A.
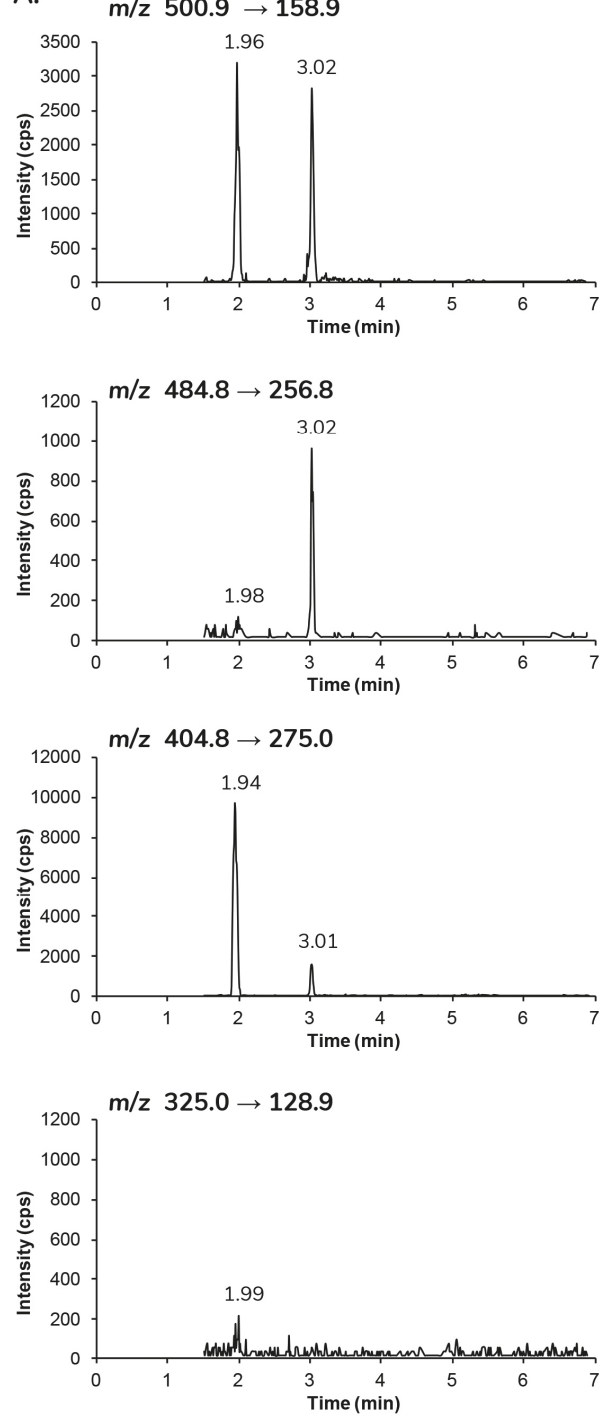

B.
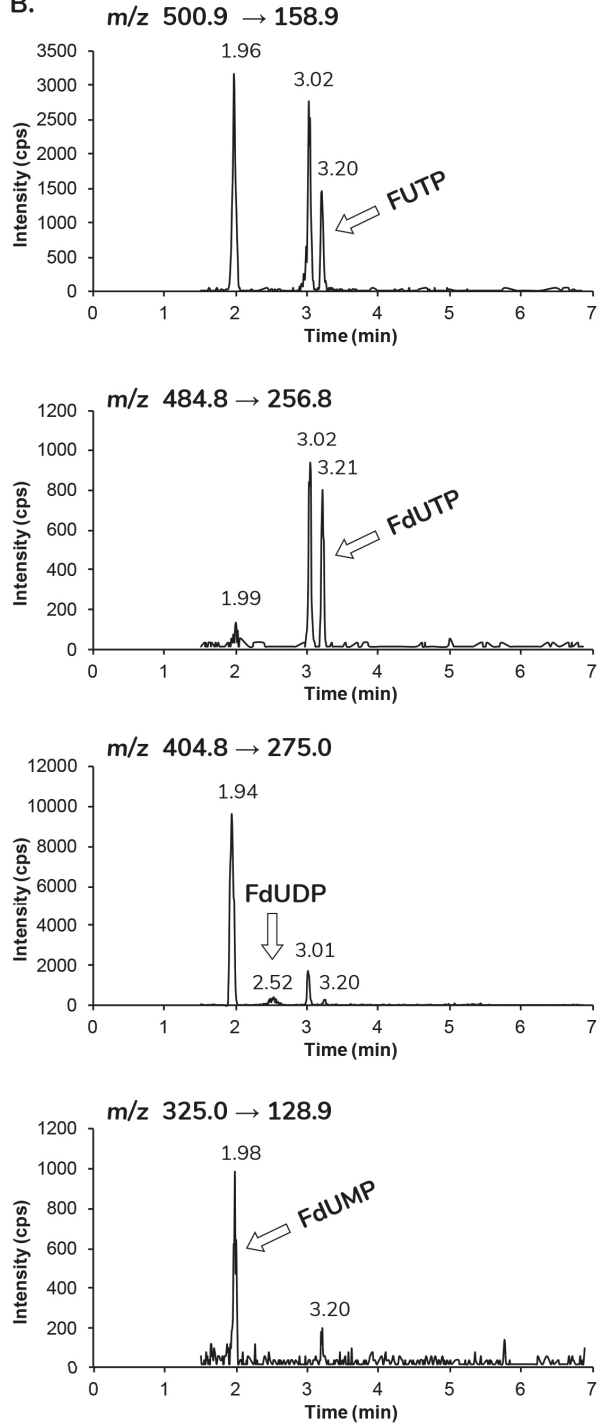

Figure 4. Representative LC-MS/MS chromatograms for blank PBMC lysate (A) and PBMC lysate spiked with FUXP and FdUXP at the lower limit of quantification (B).

and FdUMP $7.66 \mathrm{nM}$ ). Each PBMC concentration was processed and analyzed in triplicate. The matrix effect was evaluated by determination of the matrix factor at each PBMC concentration. To this end, the mean peak area found for samples with a particular PBMC concentration was divided by the mean peak 


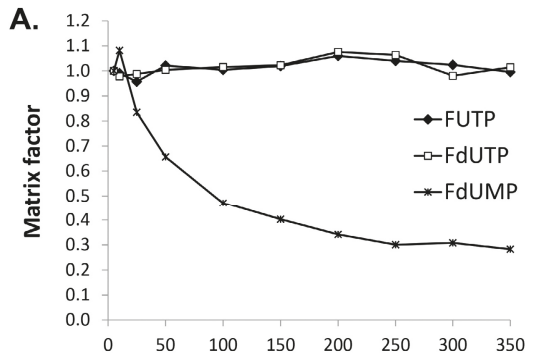

PBMC concentration ( $\times 10^{6} \mathrm{PBMCs} / \mathrm{mL}$ )

c.

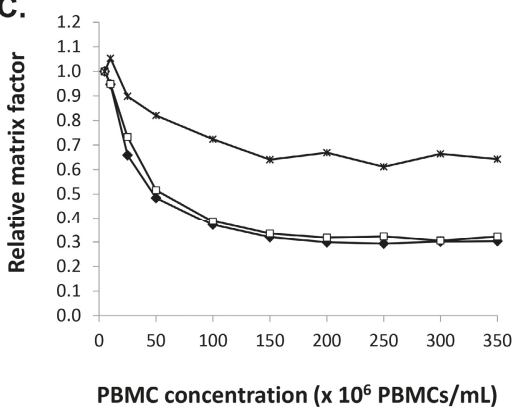

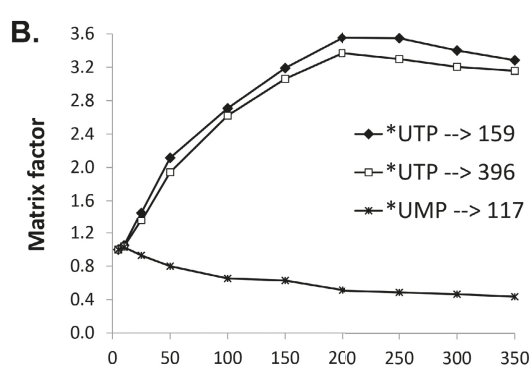

PBMC concentration ( $\times 10^{6}$ PBMCs $/ \mathrm{mL}$ )

Figure 5. Matrix factors for the analytes $(A)$ and potential internal standards $(B)$ and the relative matrix factors corrected for the internal standards (C) plotted for samples with different PBMC concentrations spiked at QC-mid-level.

area found for samples with the lowest PBMC concentration $\left(5 \times 10^{6}\right.$ PBMCs/ $\mathrm{mL}$ ) containing very little cell matrix.

In Fig. 5A the matrix factor for each analyte is displayed for different cell concentrations. The matrix factors for FUTP and FdUTP were independent of the number of PBMCs in the sample. In contrast, the matrix factor for FdUMP was strongly dependent on the number of PBMCs and decreased with increasing cell concentrations.

The matrix effects at different cell concentrations were also examined for the potential internal standards (Fig. 5B). Because isotopically labeled FUTP and FdUTP were not commercially available, ${ }^{13} \mathrm{C}_{9}{ }^{15} \mathrm{~N}_{2}$-labeled uridine 5'-triphosphate (*UTP) was tested as a potential internal standard. However, the *UTP peak areas were found to increase dramatically with the number of cells. This was a consequence of an endogenous interference at the retention time of *UTP. In 
the calibration samples, with $42 \times 10^{6} \mathrm{PBMCs} / \mathrm{mL}$, this endogenous interference caused a small peak whose contribution was negligible, based on the signal-tonoise ratio. However, at higher cell concentrations, the endogenous contribution increased and was no longer insignificant. For this reason, *UTP was not suitable for use as an internal standard in this assay.

Despite an extensive search, we did not find another suitable internal standard that eluted near or at the same retention time as FUTP and FdUTP and was not affected by interference. However, because the matrix effects for FUTP and FdUTP remained constant for different cell numbers, the use of an internal standard was not necessarily required for quantitative determination of these analytes. We therefore decided not to use an internal standard for the quantification of FUTP and FdUTP.

For FdUMP on the other hand, the matrix factor was dependent on the amount of cells in the sample. Correction by means of an internal standard was therefore necessary for FdUMP. Because no endogenous interference was observed for *UMP, this compound was selected as the internal standard. The *UMP areas slightly decreased with increasing cell concentrations (Fig. 5B). In Fig. $5 \mathrm{C}$, the relative matrix factors, corrected for the potential internal standards, are depicted. Although the relative matrix factor for FdUMP still decreased at higher cell amounts, the internal standard in a certain extent corrected for the decrease in the signal with the increase of the number of cells. We therefore decided to use *UMP as the internal standard for the quantification of FdUMP.

To further optimize the accuracy of the FdUMP quantification, it is possible to correct for the remaining matrix effect based on the number of PBMCs measured in a sample. To this end, for each PBMC concentration, a correction factor was determined by dividing the nominal FdUMP concentration by the measured concentration (Table 2). To correct for the remaining matrix effect, the measured FdUMP concentration in a sample should be multiplied by the correction factor associated with that particular cell concentration. 
Table 2. To correct for the relative matrix effect associated with a particular PBMC concentration, the measured FdUMP concentration in a sample should be multiplied by the experimentally determined correction factor.

\begin{tabular}{llll}
\hline $\begin{array}{l}\text { PBMC } \\
\text { concentration } \\
\left(\times 10^{6} \text { PBMCs } / \mathrm{mL}\right)\end{array}$ & $\begin{array}{l}\text { Nominal } \\
\text { FdUMP conc. } \\
(\mathrm{nM})\end{array}$ & $\begin{array}{l}\text { Measured } \\
\text { FdUMP conc. } \\
(\mathrm{nM})\end{array}$ & $\begin{array}{l}\text { Correction factor } \\
\text { (=nominal / measured } \\
\text { FdUMP conc.) }\end{array}$ \\
\hline 5 & 7.66 & 8.89 & 0.86 \\
10 & 7.66 & 9.35 & 0.82 \\
25 & 7.66 & 7.97 & 0.96 \\
50 & 7.66 & 7.27 & 1.05 \\
100 & 7.66 & 6.41 & 1.20 \\
150 & 7.66 & 5.64 & 1.36 \\
200 & 7.66 & 5.92 & 1.30 \\
250 & 7.66 & 5.40 & 1.42 \\
300 & 7.66 & 5.86 & 1.31 \\
350 & 7.66 & 5.67 & 1.35 \\
\hline
\end{tabular}

a For each PBMC concentration, QC mid samples were processed and analyzed in triplicate. The measured FdUMP concentration is the mean of the three measurements.

\subsection{Validation of the assay}

\subsubsection{Linearity}

Six non-zero calibration standards with the concentrations mentioned in section 2.4. were prepared and analyzed in duplicate in three separate analytical runs. For FUTP and FdUTP, calibration curves were constructed using linear regression of the analyte peak area with a $1 / x$ weighting factor (where $x=$ concentration). For FdUMP, the analyte-internal standard peak area ratio was used. For all analytes, the lower limit of quantification (LLQ) was set at the lowest calibration standard. Linearity was evaluated based on the back-calculated concentrations of the calibration standards. Deviations from the nominal concentrations should be within $\pm 20 \%$ at the LLQ and within $\pm 15 \%$ at other concentrations. No more than $25 \%$ of the calibration standards were allowed to be rejected from the calibration curves. 
The assay was linear over the validated concentration ranges in PBMC lysate from 0.488 to $19.9 \mathrm{nM}$ for FUTP, from 1.66 to $67.7 \mathrm{nM}$ for FdUTP and from 0.748 to $30.7 \mathrm{nM}$ for FdUMP. The mean back-calculated concentrations did not deviate more than $-3.3 \%$ and $+4.4 \%$ from the nominal concentrations for all compounds at all levels. The coefficients of variation ( $C V s$ ) were less than $10.5 \%$, and the correlation coefficients $(r)$ were at least 0.9948 .

\subsubsection{Accuracy and precision}

Validation samples at mid-level (FUTP 4.98 nM, FdUTP 16.9 nM and FdUMP $7.66 \mathrm{nM}$ ) were analyzed in five replicates in three analytical runs. For each compound, the intra- and inter-assay accuracy and precision values were assessed. The accuracies (both intra- and inter-assay) were between -2.2 and $7.0 \%$ deviation, and the precision values were lower than $4.9 \% \mathrm{CV}$ for all analytes. The dilution integrity was demonstrated by diluting validation samples above the upper limit of quantification (FUTP 99.6 nM, FdUTP 339 nM and FdUMP $153 \mathrm{nM}$ ) with blank PBMC lysate. A $50 \mu \mathrm{L}$ aliquot of validation sample was diluted with $950 \mu \mathrm{L}$ blank PBMC lysate in five replicates.

\subsubsection{Selectivity}

To determine the selectivity of the method, PBMCs were isolated from the blood of six different individuals who did not use 5-FU or capecitabine. The obtained blank samples were checked for interferences. No interferences were observed at the retention times of the analytes or internal standard.

\subsubsection{Carry-over}

Carry-over was determined by injecting a blank sample after the highest calibration standard and was less than $19.7 \%$ of the peak area of an LLQ sample for all analytes. Carry-over of the internal standard was less than $3.7 \%$ of the internal standard peak area of a spiked sample. 


\subsubsection{Stability}

Stability of the stock solutions was assessed after one year of storage at -70 ${ }^{\circ} \mathrm{C}$. To this end, the nucleotide stock solutions were re-quantified on the basis of freshly prepared nucleoside reference standards, using the HPLC-UV system described in section 2.2. After one year of storage, the concentrations of the stock solutions did not deviate more than $\pm 2.7 \%$ from the concentrations determined at $\mathrm{t}=0$.

Stability in PBMC lysate was evaluated using QC mid samples (FUTP 4.98 nM, FdUTP $16.9 \mathrm{nM}$ and FdUMP $7.66 \mathrm{nM})$, which were analyzed after $3 \mathrm{~h}$ storage in an ice water bath, after $24 \mathrm{~h}$ in the autosampler at $4{ }^{\circ} \mathrm{C}$ and after one month storage at $-70^{\circ} \mathrm{C}$. After storage, the QC samples were analyzed against a calibration curve obtained from freshly spiked calibration standards. The obtained concentrations did not deviate more than $\pm 14.0 \%$ from the nominal value, indicating acceptable stability.

\subsection{Clinical application of the assay}

The described assay was applied for the determination of the 5-FU nucleotide concentrations in PBMC samples obtained from three patients treated with capecitabine. These patients participated in a phase I/II study in advanced gastric cancer. This study was approved by the Medical Ethics Committee of our Institute, and all patients provided written informed consent before enrollment. Patients received capecitabine at $850 \mathrm{mg} / \mathrm{m}^{2}$ BID for fourteen days, every three weeks. Capecitabine tablets were taken with water within 30 min after a meal. Blood samples were collected on day one of the first treatment cycle, just before oral administration of capecitabine (pre-dose) and 2, 4, 6 and $8 \mathrm{~h}$ after oral capecitabine administration. Sampling times were chosen considering the plasma pharmacokinetics of capecitabine. When capecitabine is ingested with food, the maximal capecitabine and 5-FU plasma concentrations are reached after approximately $2 \mathrm{~h}$. The terminal elimination half-lives of capecitabine and its 5-FU metabolite are less than $1 \mathrm{~h}$ [22]. After collection, blood samples were immediately placed on ice, and PBMCs were isolated and processed within 30 $\min$. 


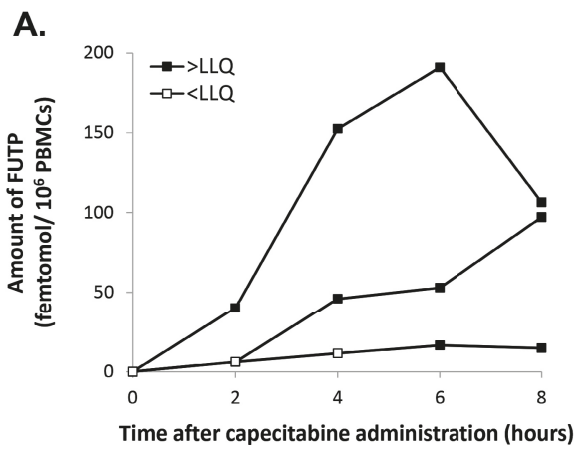

B.

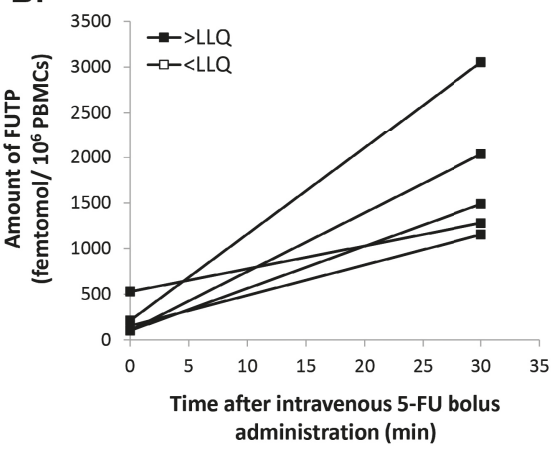

C.

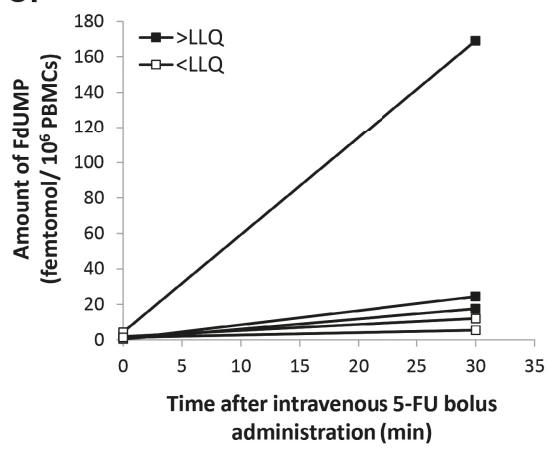

Figure 6. FUTP amounts per $10^{6} \mathrm{PBMCs}$ determined for 3 patients after a single oral capecitabine dose of $850 \mathrm{mg} / \mathrm{m}^{2}(\mathrm{~A})$; and FUTP and FdUMP amounts per $10^{6} \mathrm{PBMCs}$ determined for 5 patients after an intravenous $5-F U$ bolus of $400 \mathrm{mg} / \mathrm{m}^{2}$ in $30 \mathrm{~min}$ ( $\mathrm{B}$ and $\mathrm{C}$ ). Concentrations within the range of the assay are indicated by solid squares, and concentrations below the LLQ are represented by open squares.

In the resulting PBMC lysates obtained from patients treated with capecitabine, FUTP concentrations up to $1.84 \mathrm{nM}$ were measured. The analytical results, expressed as $\mathrm{nM}$ in PBMC lysate, were multiplied by the lysate sample volume to obtain the absolute 5-FU nucleotide amounts in a sample. These amounts were then divided by the number of cells present in the sample to obtain the 5-FU nucleotide amounts per $10^{6}$ PBMCs. The determined FUTP amounts per $10^{6}$ PBMCs are presented in Fig. 6A and show considerable variability. The FdUTP and FdUMP concentrations in these patient samples were below the LLQ of the assay. This finding was not surprising because deoxyribonucleotide concentrations in cells are several orders of magnitude lower than ribonucleotide concentrations $[23,24]$. This makes sense given that deoxyribonucleotides are 
intended for incorporation into DNA, whereas ribonucleotides are intended for incorporation into RNA, and the DNA content of a cell is lower than the total RNA content $[25,26]$.

Because potentially higher intracellular 5-FU nucleotide levels were expected after intravenous administration of 5-FU, we also collected blood samples from five patients receiving $5-\mathrm{FU}$ intravenously. Patients received a modified FOLFOX6 regimen consisting of sequentially: oxaliplatin $\left(85 \mathrm{mg} / \mathrm{m}^{2}\right.$ as a $2 \mathrm{~h}$ infusion), leucovorin (400 mg/m² as a $30 \mathrm{~min}$ infusion), a $5-\mathrm{FU}$ bolus (400 mg/ $\mathrm{m}^{2}$ in $30 \mathrm{~min}$ ), and a continuous $5-\mathrm{FU}$ infusion ( $2400 \mathrm{mg} / \mathrm{m}^{2}$ as a $44 \mathrm{~h}$ infusion), in the first $48 \mathrm{~h}$ of a 14 -day treatment cycle. Blood samples were collected on day one of the second, third or fourth treatment cycle, pre-dose and $30 \mathrm{~min}$ after the end of the 5-FU bolus.

In the resulting PBMC lysates obtained after intravenous 5-FU, FUTP concentrations up to $178 \mathrm{nM}$ were measured. In these samples, FdUMP was measured in concentrations up to $3.39 \mathrm{nM}$. Only the free FdUMP fraction is measured here because the FdUMP portion that is covalently bound to the enzyme thymidylate synthase precipitates during the extraction step with methanol $[12,27]$. The FUTP and FdUMP amounts per $10^{6}$ PBMCs are presented in Fig. 6B and C, respectively. The FdUTP concentrations in the samples obtained after intravenous administration of 5-FU were still below the LLQ of the assay.

\section{CONCLUSION}

A sensitive LC-MS/MS method for the simultaneous quantification of FUTP, FdUTP and FdUMP in PBMC lysate was developed. The best method for cell lysis and nucleotide extraction was carefully selected, and the effect of different cell numbers was examined. The assay can be applied for the measurement of the intracellular FUTP concentrations in patient samples obtained after intravenous administration of 5-FU as well as after oral administration of its prodrug capecitabine. More sensitivity may be needed for the measurement of FdUTP in patient samples. FdUMP was only measurable with this assay 
after intravenous administration of 5-FU. This is the first report in the literature where 5-FU nucleotide concentrations could be measured directly in cells from patients treated with 5-FU or capecitabine without using a radioactive label. The clinical implications of these intracellular findings will become apparent after sampling more patients and will be discussed in a future paper. 


\section{REFERENCES}

[1] Weckbecker G. Biochemical pharmacology and analysis of fluoropyrimidines alone and in combination with modulators. Pharmacol Ther 1991;50:367-424.

[2] Grem J. Mechanisms of Action and Modulation of Fluorouracil. Semin Radiat Oncol 1997;7:249-259.

[3] Álvarez P, Marchal JA, Boulaiz H, et al. 5-Fluorouracil derivatives: a patent review. Expert Opin Ther Pat 2012;22:107-23.

[4] Bazzoli C, Jullien V, Le Tiec C, et al. Intracellular pharmacokinetics of antiretroviral drugs in HIV-infected patients, and their correlation with drug action. Clin Pharmacokinet 2010;49:17-45.

[5] Jansen RS, Rosing $H$, Schellens JHM, et al. Mass spectrometry in the quantitative analysis of therapeutic intracellular nucleotide analogs. Mass Spectrom Rev 2011;30:321-43.

[6] Derissen EJB, Hillebrand MJX, Rosing H, et al. Quantitative determination of azacitidine triphosphate in peripheral blood mononuclear cells using liquid chromatography coupled with high-resolution mass spectrometry. J Pharm Biomed Anal 2014;90:7-14.

[7] Jansen RS, Rosing $H$, Schellens JHM, et al. Simultaneous quantification of $2^{\prime}, 2^{\prime}$-difluorodeoxycytidine and 2',2'-difluorodeoxyuridine nucleosides and nucleotides in white blood cells using porous graphitic carbon chromatography coupled with tandem mass spectrometry. Rapid Commun Mass Spectrom 2009;23:3040-50.

[8] Crauste C, Lefebvre I, Hovaneissian M, et al. Development of a sensitive and selective LC/MS/MS method for the simultaneous determination of intracellular 1-beta-Darabinofuranosylcytosine triphosphate (araCTP), cytidine triphosphate (CTP) and deoxycytidine triphosphate (dCTP) in a human follicular lymphoma cell line. $J$ Chromatogr B Analyt Technol Biomed Life Sci 2009;877:1417-25.

[9] Barberi-Heyob M, Merlin JL, Webér B. Determination of 5 -fluorouracil and its main metabolites in plasma by high-performance liquid chromatography. J Chromatogr 1992;573:247-52.
[10] Procházková A, Liu S, Friess $\mathrm{H}$, et al. Determination of 5-fluorouracil and 5-fluoro2'-deoxyuridine-5'-monophosphate in pancreatic cancer cell line and other biological materials using capillary electrophoresis. J Chromatogr A 2001;916:215-224.

[11] Lutz NW, Hull WE. Assignment and $\mathrm{pH}$ dependence of the 19F-NMR resonances from the fluorouracil anabolites involved in fluoropyrimidine chemotherapy. NMR Biomed 1999;12:237-48.

[12] Pogolotti A, Nolan P, Santi D. Methods for the complete analysis of 5-fluorouracil metabolites in cell extracts. Anal Biochem 1981;117:178-86.

[13] Peters GJ, Lankelma J, Kok RM, et al. Prolonged retention of high concentrations of 5 -fluorouracil in human and murine tumors as compared with plasma. Cancer Chemother Pharmacol 1993;31:269-76.

[14] Van der Wilt CL, Pinedo HM, Smid K, et al. Elevation of thymidylate synthase following 5 -fluorouracil treatment is prevented by the addition of leucovorin in murine colon tumors. Cancer Res 1992;52:4922-8.

[15] Peters GJ, Laurensse E, Steinbusch HW, et al. Development, characterization and application of an antibody against 5-fluoro2'deoxyuridine-5'monophosphate, the active metabolite of 5-fluorouracil. Anticancer Res 1993;13:835-9.

[16] Carli D, Honorat M, Cohen S, et al. Simultaneous quantification of 5-FU, 5-FUrd, 5-FdUrd, 5-FdUMP, dUMP and TMP in cultured cell models by LC-MS/MS. $J$ Chromatogr B Analyt Technol Biomed Life Sci 2009;877:2937-44.

[17] Cattel L, Airoldi M, Delprino L, et al. Pharmacokinetic evaluation of gemcitabine and 2',2'-difluorodeoxycytidine-5'triphosphate after prolonged infusion in patients affected by different solid tumors. Ann Oncol 2006;17 Suppl 5:v142-7.

[18] Yamauchi T, Negoro E, Kishi S, et al. Intracellular cytarabine triphosphate production correlates to deoxycytidine kinase/ cytosolic 5'-nucleotidase II expression ratio in primary acute myeloid leukemia cells. Biochem Pharmacol 2009;77:1780-6. 
[19] Jansen RS, Rosing $H$, Kromdijk W, et al. Simultaneous quantification of emtricitabine and tenofovir nucleotides in peripheral blood mononuclear cells using weak anion-exchange liquid chromatography coupled with tandem mass spectrometry. J Chromatogr B Analyt Technol Biomed Life Sci 2010;878:621-7.

[20] Veltkamp SA, Hillebrand MJX, Rosing $H$, et al. Quantitative analysis of gemcitabine triphosphate in human peripheral blood mononuclear cells using weak anionexchange liquid chromatography coupled with tandem mass spectrometry. J Mass Spectrom 2006;41:1633-42.

[21] Becher F, Pruvost A, Gale J, et al. A strategy for liquid chromatography/tandem mass spectrometric assays of intracellular drugs: application to the validation of the triphosphorylated anabolite of antiretrovirals in peripheral blood mononuclear cells. J Mass Spectrom 2003;38:879-90.

[22] Reigner B, Blesch K, Weidekamm E. Clinical pharmacokinetics of capecitabine. Clin Pharmacokinet 2001;40:85-104.

[23] Chen P, Liu Z, Liu S, et al. A LC-MS/MS method for the analysis of intracellular nucleoside triphosphate levels. Pharm Res 2009;26:1504-15.

[24] Cohen S, Megherbi M, Jordheim LP, et al. Simultaneous analysis of eight nucleoside triphosphates in cell lines by liquid chromatography coupled with tandem mass spectrometry. J Chromatogr B Analyt Technol Biomed Life Sci 2009;877:3831-40.

[25] Cohen S, Jordheim LP, Megherbi M, et al. Liquid chromatographic methods for the determination of endogenous nucleotides and nucleotide analogs used in cancer therapy: a review. J Chromatogr B Analyt Technol Biomed Life Sci 2010;878:1912-28.

[26] Malacinski GM. Essentials of Molecular Biology. fourth ed. Jones \& Bartlett Learning, 2005.

[27] Washtien WL, Santi D V. Assay of intracellular free and macromolecular-bound metabolites of 5-fluorodeoxyuridine and 5-fluorouracil. Cancer Res 1979;39:3397-404. 


\section{CHAPTER 3.2}

\section{Exploring the intracellular pharmacokinetics of the 5-fluorouracil nucleotides during capecitabine treatment}

British Journal of Clinical Pharmacology 2016;81:949-957.

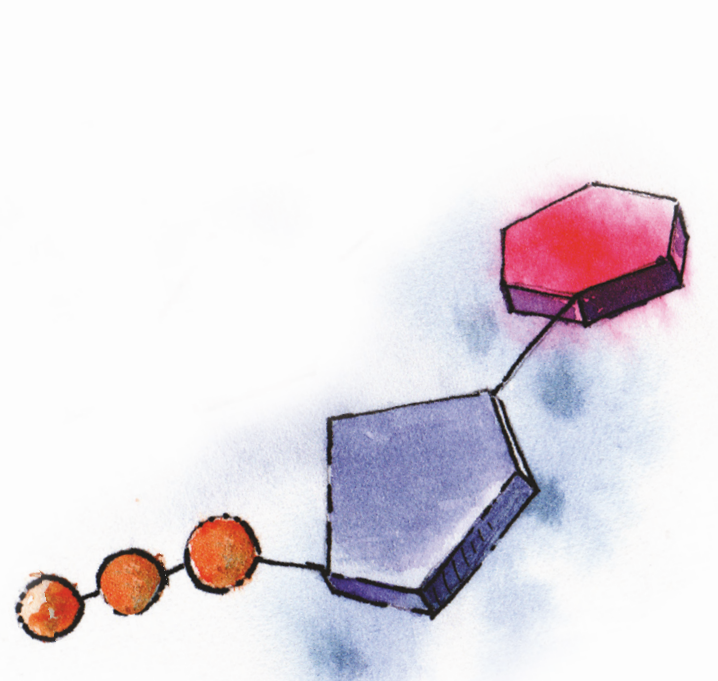

Ellen J.B. Derissen

Bart A.W. Jacobs

Alwin D.R. Huitema Hilde Rosing Jan H.M. Schellens Jos H. Beijnen 


\section{ABSTRACT}

AIM Three intracellularly formed metabolites are responsible for the antineoplastic effect of capecitabine: 5-fluorouridine 5'-triphosphate (FUTP), 5-fluoro-2'-deoxyuridine 5'-triphosphate (FdUTP), and 5-fluoro-2'-deoxyuridine 5'-monophosphate (FdUMP). The objective of this study was to explore the pharmacokinetics of these intracellular metabolites during capecitabine treatment.

METHODS Serial plasma and peripheral blood mononuclear cell (PBMC) samples were collected from 13 patients treated with capecitabine $1000 \mathrm{mg}$ QD (group A) and eight patients receiving capecitabine $850 \mathrm{mg} / \mathrm{m}^{2}$ BID for fourteen days, every three weeks (group B). Samples were collected on day 1 and, for four patients of group B, also on day 14 . The capecitabine and 5-fluorouracil (5-FU) plasma concentrations and intracellular metabolite concentrations were determined using LC-MS/MS. Pharmacokinetic parameters were estimated using non-compartmental analysis.

RESULTS Only FUTP could be measured in the PBMC samples. The FdUTP and FdUMP concentrations were below the detection limits (LOD). No significant correlation was found between the plasma 5-FU and intracellular FUTP exposure. The FUTP concentration-time profiles demonstrated considerable inter-individual variation and accumulation of the metabolite in PBMCs. FUTP levels ranged between $\angle L O D$ and $1.0 \mu \mathrm{M}$ on day 1 , and from 0.64 to $14 \mu \mathrm{M}$ on day 14 . The area under the FUTP concentration-time curve was significantly increased on day 14 of the treatment compared to day 1 (mean \pm SD: $28 \pm 19$ $\mu M^{*} h$ vs. $\left.2.0 \pm 1.9 \mu M^{*} h\right)$.

CONCLUSIONS To our knowledge, this is the first time that intracellular FUTP concentrations were measured in patients treated with capecitabine. During 14 days of treatment with capecitabine twice daily, intracellular accumulation of FUTP occurs.

Keywords: 5-fluorouracil; 5-fluorouridine triphosphate (FUTP); capecitabine; nucleotides; peripheral blood mononuclear cells (PBMCs); pharmacokinetics. 


\section{INTRODUCTION}

Capecitabine is a widely used chemotherapeutic agent, which has an important place in the treatment of several malignancies, including colorectal, gastric, pancreatic, breast and head and neck cancer. It was developed as a tumourselective prodrug of 5-fluorouracil (5-FU). After oral administration, capecitabine is extensively absorbed from the gastrointestinal tract, and then converted into 5-FU by an enzymatic cascade involving three steps (Fig. 1). First, capecitabine is converted to 5'-deoxy-5-fluorocytidine (5'-dFCR) by carboxylesterase, an enzyme located primarily in the liver. 5'-dFCR is then converted to 5'-deoxy5-fluorouridine (5'-dFUR) by cytidine deaminase, which is principally located in the liver and in tumour tissue. The third step, the conversion of 5'-dFUR to 5-FU is catalysed by thymidine phosphorylase. This enzyme is present at higher concentrations in solid tumour tissue than in normal tissues [1]. Therefore, the third activation step preferentially takes place in tumour tissue rather than normal tissue [2].

5-FU is further activated intracellularly by ribosylation and sequential phosphorylation. Ultimately, three intracellularly formed metabolites (nucleotides) are held responsible for the antineoplastic effect of capecitabine. These are 5-fluorouridine 5'-triphosphate (FUTP), 5-fluoro-2'-deoxyuridine 5'-triphosphate (FdUTP), and 5-fluoro-2'-deoxyuridine 5'-monophosphate (FdUMP). In brief, FUTP is incorporated into RNA and interferes with normal RNA processing and function. FdUTP is incorporated into DNA, leading to DNA damage and ultimately cell death. FdUMP inhibits thymidylate synthase, the enzyme that catalyses the transformation of deoxyuridine monophosphate (dUMP) to deoxythymidine monophosphate (dTMP). Inhibition of thymidylate synthase by FdUMP leads to accumulation of deoxyuridine triphosphate (dUTP) and depletion of deoxythymidine triphosphate (dTTP). This imbalance has deleterious consequences for DNA synthesis and repair, ultimately leading to cell death (Fig. 1) [3,4].

As the anticancer activity of capecitabine depends on these three 5-FU nucleotides, it would be interesting and clinically relevant to monitor their intracellular concentrations during capecitabine treatment. This would provide insight into the amounts of activated drug reaching the site of action. This 


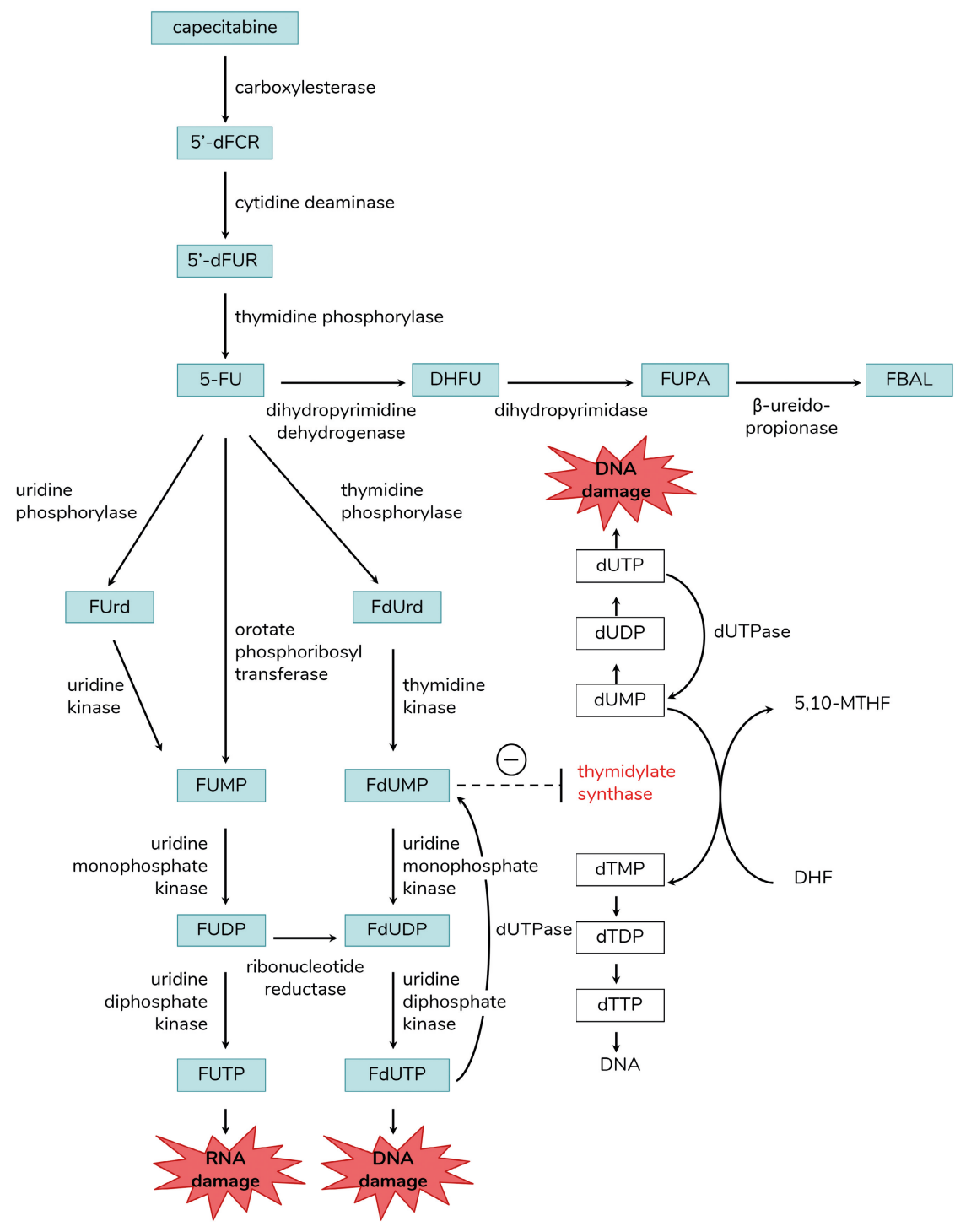

Figure 1. Capecitabine metabolism and mechanisms of action

Abbreviations: 5'-dFCR, 5'-deoxy-5-fluorocytidine; 5'-dFUR, 5'-deoxy-5-fluorouridine; DHFU, dihydrofluorouracil; FUPA, a-fluoro- $\beta$-ureidopropionic acid; FBAL, a-fluoro- $\beta$-alanine; 5-FU, 5-fluorouracil; FUrd, 5-fluorouridine; FdUrd, 5-fluoro-2'-deoxyuridine; FUMP, 5-fluorouridine 5'-monophosphate; FUDP, 5-fluorouridine 5'-diphosphate; FUTP, 5-fluorouridine 5'-triphosphate; FdUMP, 5-fluoro-2'-deoxyuridine 5'-monophosphate; FdUDP, 5-fluoro-2'-deoxyuridine 5'-diphosphate; FdUTP, 5-fluoro-2'-deoxyuridine 5'-triphosphate; dUMP, 2'-deoxyuridine 5'-monophosphate; dUDP, 2'-deoxyuridine 5'-diphosphate; dUTP, 2'-deoxyuridine 5'-triphosphate; dTMP, 2'-deoxythymidine 5'-monophosphate; dTDP, 2'-deoxythymidine 5'-diphosphate; dTTP, 2'-deoxythymidine 5'-triphosphate; DHF, dihydrofolate; 5,10-MTHF, 5,10-methylene-tetrahydrofolate. 
information could ultimately be very useful to optimize current treatment regimens.

Until now, information about the intracellular metabolism of the 5-FU nucleotides originated mainly from in vitro experiments and animal studies [5-8]. Little is known about the formation of 5-FU nucleotides in patients who are treated with 5-FU due to the long lack of a suitable bioanalytical assay.

Several studies were conducted that examined the FdUMP concentrations in tumour tissues of patients who were treated with an intravenous 5-FU bolus injection $\left(500 \mathrm{mg} / \mathrm{m}^{2}\right)$ [9-11]. These studies used a competitive-binding assay with thymidylate synthase, isolated from Lactobacillus casei, as a binding protein. The quantification of FdUMP was based on the displacement of a known amount of radiolabelled $\left[{ }^{3} \mathrm{H}\right]$-FdUMP, measured by scintillation counting [12].

However, for the quantification of FUTP and FdUTP in cells of patients who were treated with 5-FU, a sufficiently sensitive assay was missing. Therefore, we recently developed an ultrasensitive liquid chromatography-tandem mass spectrometry (LC-MS/MS) assay for the quantification of the active 5-FU nucleotides in peripheral blood mononuclear cells (PBMCs) [13]. PBMCs were selected as a cell model for the intracellular activation, because they are easy to collect at various time points after drug administration. With the advent of this assay, it has become possible to get insight into the intracellular 5-FU nucleotide concentrations in samples of patients who are treated with 5-FU or capecitabine.

The aim of the current study was to explore the intracellular pharmacokinetics (PK) of the three pharmacologically active 5-FU nucleotides during capecitabine treatment. Except for a small pilot in our previous publication on the development of a bioanalytical assay [13], this is, to our knowledge, the first time that intracellular 5-FU nucleotides were quantified during capecitabine treatment. 


\section{METHODS}

\section{Study design and treatment schedule}

The intracellular PK of the active 5-FU nucleotides was assessed in two groups of patients. For group A, the intracellular PK of the 5-FU nucleotides was studied only on day 1 of the treatment. Based on the results in this group, we wondered if intracellular accumulation would occur during a treatment cycle, in which capecitabine is administered twice daily for 14 consecutive days. Therefore group B was added to the study. For this group the intracellular PK was examined on day 1 and also on day 14 of the treatment with capecitabine.

Group A included 13 patients treated with capecitabine for one of its approved therapeutic indications (e.g. colon, breast, pancreatic and gastric cancer). All patients received a one-time capecitabine dose of $1000 \mathrm{mg}$ QD to study the PK, and were then treated with a standard dose. Patients were instructed not to eat or drink (except a small amount of water $<50 \mathrm{~mL}$ ) from 11 hours before the drug intake until 1 hour after drug intake. PBMC samples were collected on day 1 of the first treatment cycle, just before oral administration of capecitabine (predose) and 1, 2, 4, 6 and $24 \mathrm{~h}$ after capecitabine administration. To monitor the capecitabine and 5-FU plasma concentrations, plasma samples were collected pre-dose and 0.5, 1, 1.5, 2, 3, 4, 6, 12 and $24 \mathrm{~h}$ after capecitabine intake.

Group B consisted of eight patients who participated in a phase I/II study in advanced gastro-oesophageal cancer. The primary objective of this study was to explore the safety and preliminary activity of the combination of docetaxel, oxaliplatin and capecitabine. Patients received capecitabine $850 \mathrm{mg} / \mathrm{m}^{2}$ twice daily for 14 days, every three weeks. Capecitabine tablets were taken with water within 30 minutes after a meal. PBMC samples were collected at day 1 of the first treatment cycle (for five patients) and at day 14 of this cycle (for four patients). PBMC samples were collected pre-dose and 2, 4, 6 and $8 \mathrm{~h}$ after oral capecitabine administration. For one patient an additional sample was taken $10 \mathrm{~h}$ after capecitabine administration. Plasma samples, to monitor the capecitabine and 5-FU plasma concentrations, were collected pre-dose and $0.5,1,2,3,4,6$ and $8 \mathrm{~h}$ after capecitabine intake. Sample collection at day 14 
of the treatment was included in the protocol to examine whether intracellular accumulation of 5-FU nucleotides occurs during a treatment cycle.

The study was approved by the Medical Ethics Committee of our Institute and was conducted in accordance with the Declaration of Helsinki. All patients provided written informed consent before enrolment.

\section{Quantification of capecitabine and 5-FU in plasma}

Capecitabine and 5-FU were quantified in plasma by the two validated LC-MS/ MS assays described by Deenen et al. [14]. In brief, stable labelled isotopes of the analytes were used as internal standards and added to $100 \mu \mathrm{L}$ of plasma, followed by a protein precipitation step. The capecitabine assay was based on reversed-phase chromatography using an XBridge C18 column (50 mm $\times 2.1 \mathrm{~mm}$ ID, particle size $5 \mu \mathrm{m}$; Waters Corporation, Milford, MA, USA) and a mobile phase gradient, in which mobile phase $A$ consisted of $0.05 \%$ formic acid in water and mobile phase B was $0.05 \%$ formic acid in methanol. For the quantification of 5-FU, the chromatography was performed on a Luna HILIC column (150 mm x $2.1 \mathrm{~mm}$ ID, particle size $3 \mu \mathrm{m}$; Phenomenex, Torrance, CA, USA) using isocratic elution with $10 \mathrm{mM}$ formic acid in water $(\mathrm{pH} 4.0)$ mixed with acetonitrile $(20: 80, v / v)$. Detection of the analytes was performed on an API4000 triple quadrupole mass spectrometer equipped with an electrospray ionization probe (AB Sciex, Framingham, MA, USA). Capecitabine was detected in the positive ion mode and 5-FU in the negative ion mode. The lower limits of quantification (LLQs) of the assays were $139 \mathrm{nM}$ for capecitabine and 384 nM for 5-FU [14].

\section{Quantification of the 5-FU nucleotides in PBMCs}

PBMCs were isolated and FUTP, FdUTP and FdUMP concentrations were determined as previously described using our validated LC-MS/MS assay [13]. In brief, $16 \mathrm{~mL}$ of blood was collected and PBMCs were isolated immediately using cold Ficoll-Paque PLUS density gradient (GE Healthcare, Pittsburgh, PA, USA). The collected PBMCs were resuspended in $70 \mu \mathrm{L}$ PBS resulting in a homogeneous cell suspension with a total volume of approximately 100 $\mu \mathrm{L}$. A $30 \mu \mathrm{L}$ aliquot of this cell suspension was used to perform a cell count 
using a haematology analyser (Cell-Dyn Sapphire; Abbott Diagnostics). A $60 \mu \mathrm{L}$ aliquot of the cell suspension was used for determination of the 5-FU nucleotide concentrations. To this end, cells were lysed by the addition of 100 $\mu \mathrm{L}$ methanol and extensive vortex mixing. After centrifugation, the supernatant (PBMC lysate) was collected and stored at $-70{ }^{\circ} \mathrm{C}$ until analysis. Directly prior to LC-MS/MS analysis, the PBMC lysates were spiked with internal standard. Chromatographic separations were performed by weak anion exchange chromatography using a Biobasic AX column $(50 \mathrm{~mm} \times 2.1 \mathrm{~mm}$ ID, particle size $5 \mu \mathrm{m}$, Thermo Scientific). A stepwise gradient was used, in which the eluent $\mathrm{pH}$ was increased and the $\mathrm{NH}_{4} \mathrm{Ac}$ concentration decreased. Detection was performed using a QTrap 5500 mass spectrometer equipped with an electrospray ionization probe operating in the negative ion mode (AB Sciex). The LLQs in PBMC lysate were $0.488 \mathrm{nM}$ for FUTP, $1.66 \mathrm{nM}$ for FdUTP and $0.748 \mathrm{nM}$ for FdUMP. Accuracies were between -2.2 and $7.0 \%$ deviation for all analytes, and the coefficient of variation values were $\leq 4.9 \%$ [13].

The analytical results, expressed as $\mathrm{nM}$ in PBMC lysate, were multiplied with the lysate sample volume to obtain the absolute 5-FU nucleotide amounts in a sample. These amounts were then divided by the number of cells present in the sample to obtain the 5-FU nucleotide amounts per $10^{6} \mathrm{PBMCs}$. Intracellular concentrations are generally expressed as the amount of drug per $10^{6}$ cells. However, in order to compare the intracellular concentrations with the plasma concentrations, the intracellular concentrations were also converted to the amount of drug per volume unit $(\mu \mathrm{M})$. To this end, we measured the number of lymphocytes, monocytes and (residual) granulocytes in each individual sample and used the mean cell volumes of lymphocytes (174 fL), monocytes (339 fL) and granulocytes (302 fL) as determined by Sharma et al. [15] and later confirmed by Simiele et al. [16].

\section{Pharmacokinetic and statistical analysis}

The individual non-compartmental PK parameters in plasma and in PBMCs were determined using validated scripts in the software package $R$ (version 3.1.2). The mean and coefficient of variation (CV) of the following PK parameters were reported: the maximum observed concentration $\left(C_{\max }\right)$, the time to reach $C_{\max }$ 
$\left(t_{\max }\right)$ and the area under the concentration-time curve between $t=0$ and the time point of the last quantifiable data point $\left(A \cup C_{0-t}\right)$.

For the plasma samples, also the terminal elimination half-life $\left(t_{1 / 2}\right)$ was calculated, using the terminal elimination rate constant $\left(k_{e}\right)$ based on the last three data points. For the calculation of the mean $t_{1 / 2}$, only curves that contained sufficient data points to assess this parameter were included. This meant that curves lacking a clear elimination phase were excluded from the calculation of this specific PK parameter. For the PBMC samples, the $t_{1 / 2}$ could not be calculated due to the limited number of data points in the descending part of the FUTP curves. The intracellular FUTP concentrations after 24 hours were reported as a percentage of the $C_{\max }$.

The relationship between the plasma 5-FU exposure and intracellular FUTP exposure was explored. Pearson's correlation was used to assess whether there was a linear relationship between the $A \cup C_{0-t}$ of $5-F U$ in plasma and the $A \cup C_{0-t}$ of FUTP measured in PBMCs. The correlation was considered statistically significant if the $p$ value was less than 0.05 .

To assess whether intracellular accumulation occurred during 14 days of capecitabine treatment twice daily, the $A_{U} \mathrm{C}_{0-8 \mathrm{~h}}$ values for FUTP determined on day 1 and on day 14 of the treatment were compared. A one-sided MannWhitney $U$ test was performed to assess (for group B) whether there was a significant increase of the $A \cup C_{0-8 \mathrm{~h}}$ values for intracellular FUTP at day 14 compared to day 1 . The result was considered statistically significant if the $p$ value was less than 0.025 . Statistical analyses were performed using SPSS Statistics version 23 (IBM Corp.). 


\section{RESULTS}

\section{Plasma pharmacokinetics}

The mean plasma concentration-time curves for capecitabine and 5-FU are shown in Fig. 2. The different shapes of the curves for group $A$ and group $B$ are probably related to whether or not the patients took food around the time of drug intake [17]. The results of the non-compartmental PK analysis are shown in Table 1. On average, the $C_{\max }$ for capecitabine was reached after 0.7 hours in patient group $A$ and after 3.3 hours in group B. This difference is probably also related to the effect of food. The mean terminal elimination half-lives of capecitabine and 5-FU were 0.4 and 0.6 hours for group $A$ and 0.7 and 0.8 hours for group $B$, respectively. These PK data are in line with previously published data in the literature [18]. The plasma concentrationtime curves determined on day 14 demonstrate (despite the limited number of sampling times and missing the $C_{\max }$ ) that there was no accumulation of 5-FU or capecitabine in plasma (Fig. $2 \mathrm{~B}$ ).

\section{Intracellular pharmacokinetics}

In the PBMC samples, only the intracellular FUTP concentrations could be quantified. The FdUTP and FdUMP concentrations in the collected PBMC lysates were all below the detection limits of the assay. The LLQs of the LC-MS/MS assay (in PBMC lysate), mentioned in the methods section, are not directly translatable to the lowest measurable intracellular FdUTP and FdUMP concentrations in PBMCs, because the latter also depends on the number of collected lymphocytes, monocytes and granulocytes in the sample. Clinical samples derived from $16 \mathrm{~mL}$ whole blood using the described isolation method were found to contain 0.5-20 × 106 PBMCs.

The intracellular concentration-time curves for FUTP are shown in Fig. 3 and demonstrate considerable inter-individual variation. The results of the noncompartmental PK analysis are given in Table 1. Intracellular FUTP circulates much longer than the corresponding plasma levels of capecitabine and 5-FU. On day 1 of the treatment, the $C_{\max }$ for FUTP was reached on average after 3.5 hours in patient group A. As for the plasma concentrations, also for the 
A.
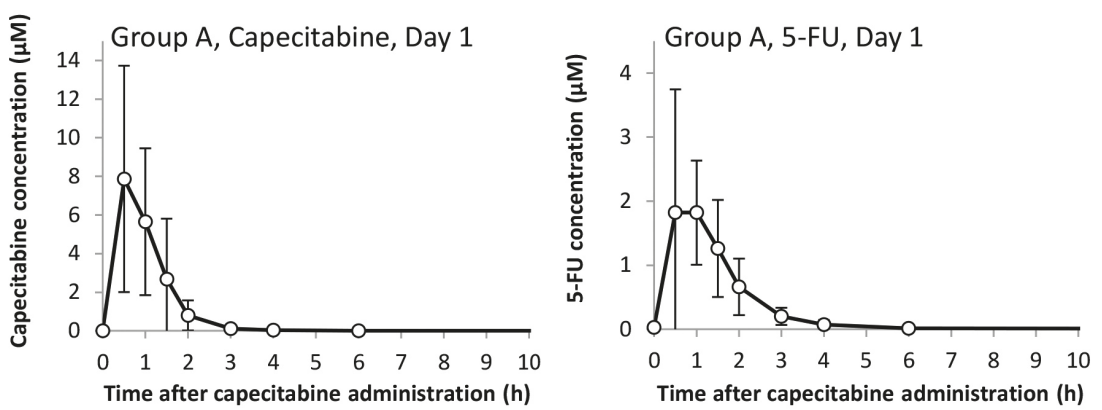

B.
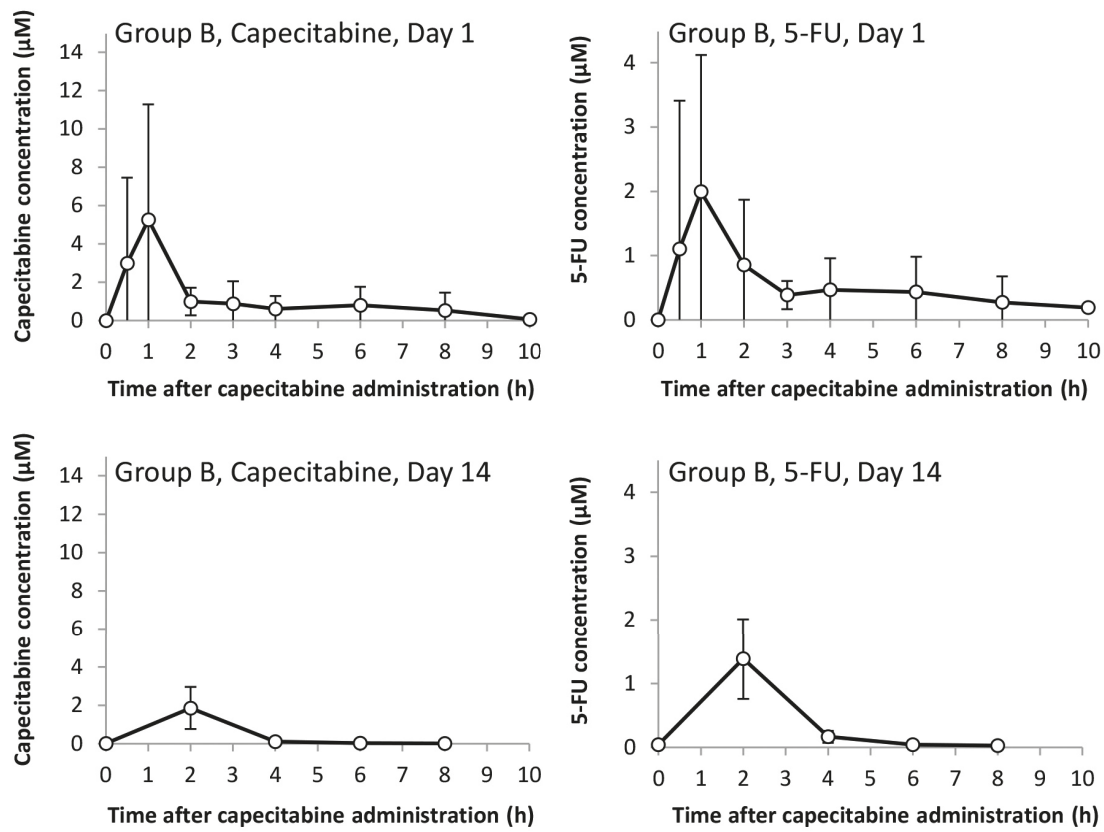

Figure 2. Plasma concentration-time curves of capecitabine and 5-FU for patient group A (receiving capecitabine $1000 \mathrm{mg}$ QD) measured at day 1 of the first treatment cycle (A), and for patient group B (receiving capecitabine $850 \mathrm{mg} / \mathrm{m}^{2} \mathrm{BID}$ ) measured at day 1 and at day 14 of the first treatment cycle (B). Despite the limited number of sampling times on day 14 and the missing $C_{\max }$, the plasma concentration-time curves determined on day 14 demonstrate that there was no accumulation of capecitabine or 5-FU in plasma. Curves were depicted up to $10 \mathrm{~h}$ after capecitabine intake, although more samples were collected for group A. The data are shown as mean values (symbols) with standard deviations (error bars). 
A.

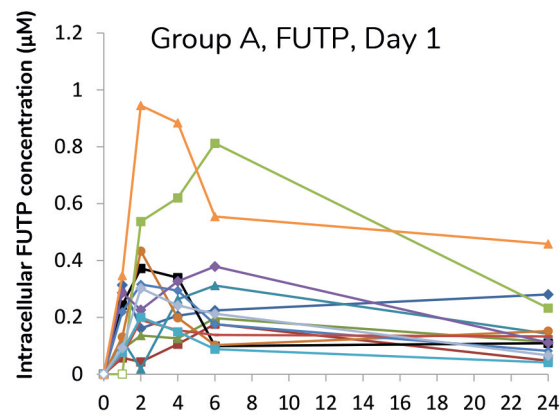

Time after capecitabine administration (hours)

B.
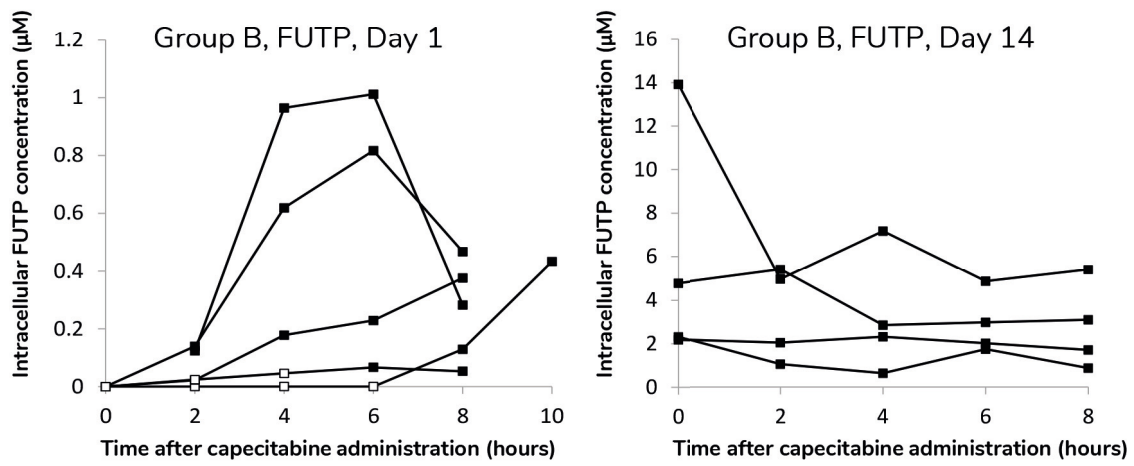

Figure 3. Intracellular concentration-time curves of 5-fluorouridine 5'-triphosphate (FUTP) for patient group A (receiving capecitabine $1000 \mathrm{mg}$ QD) measured at day 1 of the first treatment cycle (A), and for patient group B (receiving capecitabine $850 \mathrm{mg} / \mathrm{m}^{2}$ BID) measured at day 1 and at day 14 of the first treatment cycle (B). Concentrations within the range of the assay are indicated by solid markers and concentrations below the LLQ by open markers.

intracellular levels, $C_{\max }$ was achieved much later in group $B$, after 7.2 hours. Remarkable is the long presence of FUTP in the cell. Twenty-four hours after the intake of capecitabine, the intracellular FUTP concentration was on average still $41 \%$ of the $\mathrm{C}_{\max }$ (range: $21-89 \%$ ). 


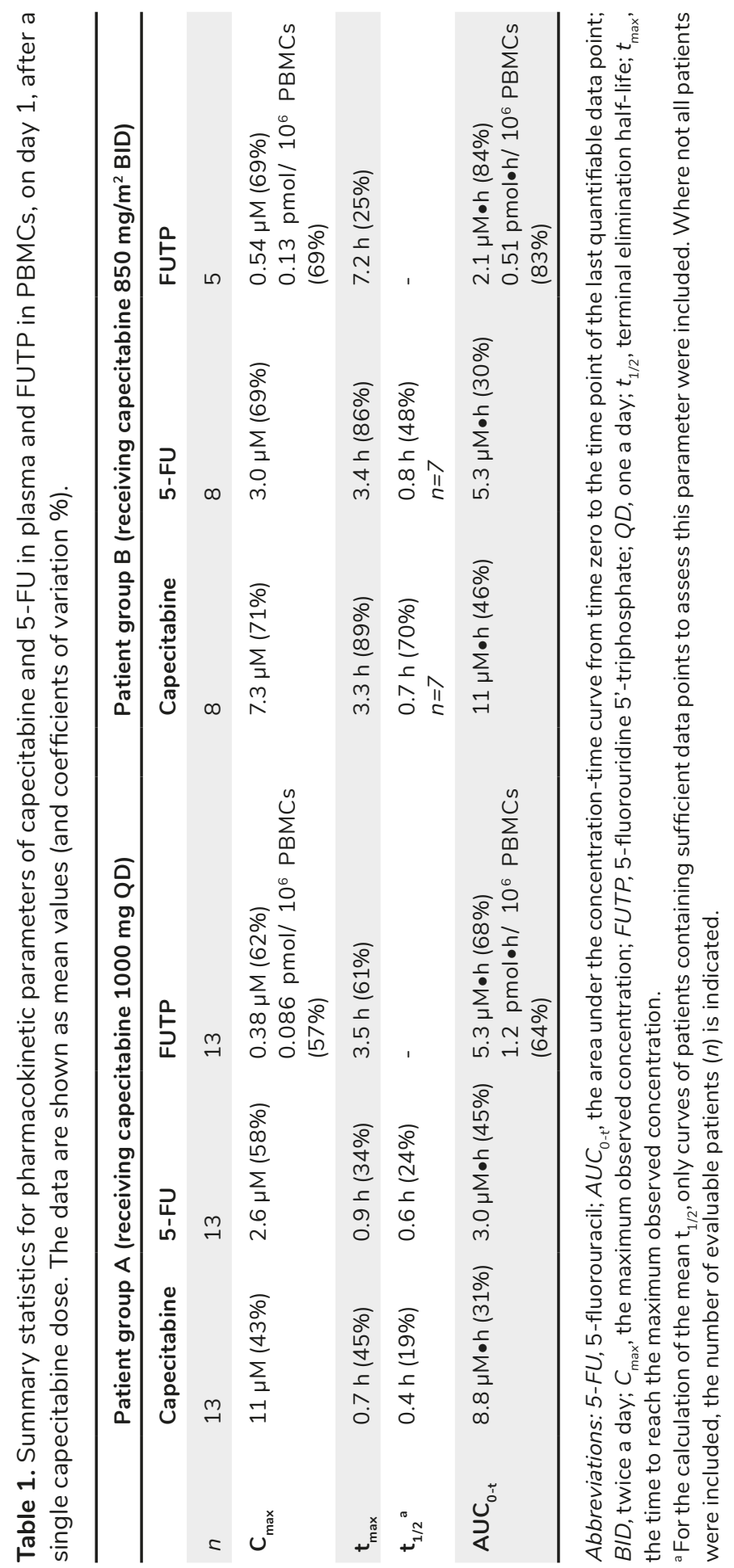




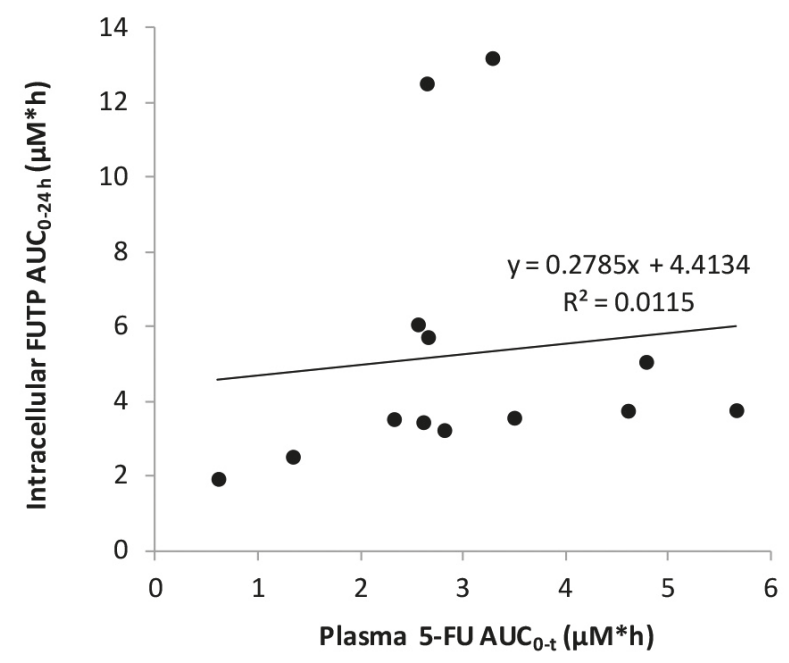

Figure 4. Relationship between the $A \cup C_{0-t}$ of $5-F U$ in plasma and the $A \cup C_{0-24 h}$ of intracellular FUTP in PBMCs determined for group $A$ on day 1 of the treatment.

\section{Relationship between plasma 5-FU and intracellular FUTP exposure}

No significant linear correlation was found between the $\mathrm{AUC}_{0-\mathrm{t}}$ of $5-\mathrm{FU}$ in plasma and the $\mathrm{AUC}_{0-24 \mathrm{~h}}$ of intracellular FUTP determined on the first treatment day (Fig. 4). The Pearson correlation coefficient ( $r$ ) was $0.107(p=.727, n=13$ ) for group $A$. The sample size of group $B$ was too small to make a reliable statement about possible linear correlation.

\section{Intracellular FUTP accumulation}

After 14 days of capecitabine treatment (twice daily), intracellular FUTP concentrations are clearly higher than after the first capecitabine administration on day 1 (Fig. 3B). The $A_{U} C_{0-8 h}$ for intracellular FUTP was significantly increased on day 14 of the treatment compared to day 1 (Mann-Whitney $U=20, p=.008$, one-sided, Fig. 5). The mean $\mathrm{AUC}_{0-8 \mathrm{~h}}\left( \pm \mathrm{SD}\right.$ ) on day 1 was $2.0 \pm 1.9 \mu \mathrm{M}^{*} \mathrm{~h}$ vs. $28 \pm 19 \mu \mathrm{M}^{*} \mathrm{~h}$ on day 14 . Apparently, during these 14 days of consecutive treatment, intracellular accumulation of FUTP occurs. 


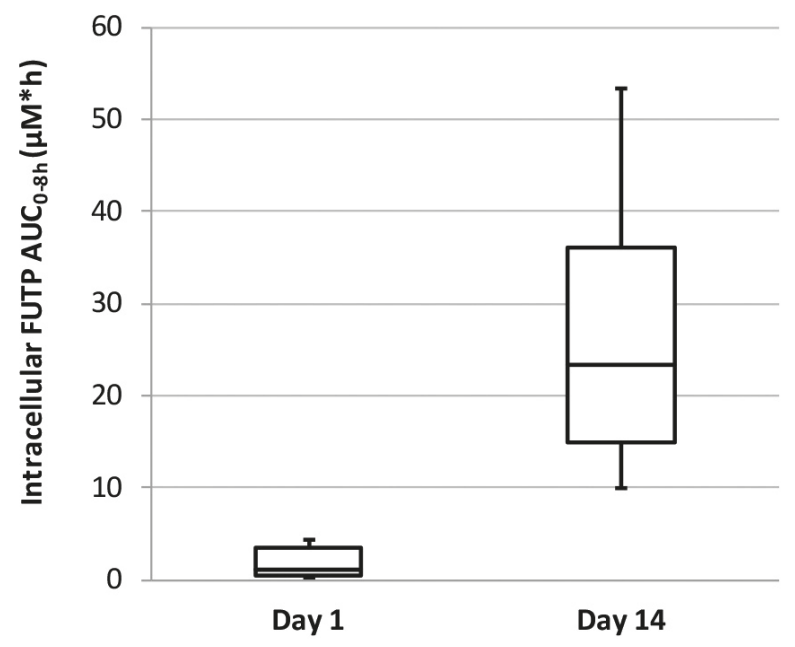

Figure 5. Box plot for the $\mathrm{AUC}_{0-8 \mathrm{~h}}$ of FUTP on day 1 and on day 14 of capecitabine treatment (twice daily). The box represents the median and the $25^{\text {th }}$ and $75^{\text {th }}$ percentiles of the data. The whiskers represent the range. The $A \cup C_{0-8 h}$ for intracellular FUTP was significantly increased on day 14 of the treatment compared to day 1 (Mann-Whitney $\mathrm{U}=20, p=.008$, one-sided).

\section{DISCUSSION}

To our knowledge, this is the first time that intracellular FUTP concentrations were measured in patients who were treated with capecitabine. Our measurements show that FUTP is present in higher concentrations than FdUTP and FdUMP (which were both below the detection limits of the assay). Apparently, capecitabine is mainly converted into FUTP and to a lesser extent into FdUTP and FdUMP. However, this does not necessarily mean that the RNA pathway has a more prominent role in the mechanism of action than the DNA pathway. It is known that the deoxyribonucleotides are naturally present within cells in much lower concentrations than the ribonucleotides $[19,20]$. This means that the low FdUTP and FdUMP concentrations may still have a very important role.

A second important finding of this exploratory intracellular PK study is the long intracellular residence of FUTP, and its consequent intracellular accumulation 
after dosing capecitabine twice daily for 14 days. The prolonged intracellular retention of FUTP is an important finding, especially in view of the rapid plasma kinetics of capecitabine and 5-FU.

It was already known that 5-FU plasma levels do not reflect the 5-FU concentrations in tumour tissue. Peters et al. found that $5-\mathrm{FU}$ was retained for a much longer period of time in tissues than in plasma, and that 4-48 $\mathrm{h}$ after an i.v. 5 -FU bolus of $500 \mathrm{mg} / \mathrm{m}^{2}$, the 5 -FU tissue concentrations were at least 10 times higher than the plasma concentrations [11].

On the basis of this exploratory study, we now know that FUTP retains in cells for at least $24 \mathrm{~h}$ after a single capecitabine dose. The prolonged retention of FUTP in (tumour) tissue must be taken into consideration when designing new dose regimens.

It would be interesting to know whether also intracellular accumulation of FdUTP and FdUMP occurs during 14 days of treatment with capecitabine, especially given that the effects of these nucleotides are more cell cycle dependent than the effect of FUTP [21]. Prolonged intracellular exposure to FdUMP and FdUTP will affect more cells during their S-phases. Unfortunately, a more sensitive assay is required to measure the intracellular FdUTP and FdUMP levels.

Capecitabine was developed as an oral alternative to continuous intravenous 5-FU infusion [22]. It is known that prolonged exposure to 5-FU has a beneficial effect compared to brief exposure. In advanced colorectal cancer, continuous intravenous infusion resulted in significantly higher response rates and less toxicity compared with intravenous bolus injections [23]. Prolonged 5-FU exposure will probably lead to a prolonged intracellular exposure to 5-FU nucleotides, which is beneficial, especially as the effects of the 5-FU nucleotides are cell cycle dependent. We wondered if prolonged 5-FU plasma levels also lead to higher intracellular nucleotide concentrations than brief exposure to an equal amount of 5-FU. This would be the case if the transporters involved in the cellular uptake or the enzymes involved in the intracellular ribosylation or phosphorylation would become saturated above a certain 5-FU concentration. Remarkably, the FUTP concentrations which we reported in our analytical paper, measured 30 minutes after an intravenous 5 -FU bolus (400 mg/m² in $30 \mathrm{~min}$ ) 
were in the same range as the FUTP concentrations measured after 14 days of capecitabine treatment twice daily (4.7-11 $\mu \mathrm{M} v s$. 0.64-14 $\mu \mathrm{M})$ [13]. This indicates that also brief, high 5-FU exposure can rapidly lead to high intracellular FUTP concentrations and the capacities of the cell membrane transporters and intracellular enzymes are probably not the limiting factors.

When interpreting the results of this intracellular PK study, we should keep in mind that the capecitabine dose that patients received during the study, 1000 mg QD for group A and $850 \mathrm{mg} / \mathrm{m}^{2}$ BID for group B, is lower than the typical dose that is used when capecitabine is given as a single agent: $1250 \mathrm{mg} / \mathrm{m}^{2}$ twice daily for 14 days followed by a 7 -day rest period.

Furthermore, it should be realized that the FdUMP fraction which is bound to thymidylate synthase is not measured by the employed assay, because this fraction is precipitated during the extraction step with methanol $[24,25]$.

In addition, we have to keep in mind that the nucleotide concentrations measured in PBMCs may be different than the concentrations that would be found in tumour cells. PBMCs were used as a cell model for the intracellular activation, because these cells can be obtained more easily than tumour biopsies. This was particularly relevant as we wanted to follow the intracellular FUTP concentrations over time. Therefore samples had to be collected at multiple time points after capecitabine intake, which was not possible for tumour tissue.

However, by measuring the 5-FU nucleotide concentrations in PBMCs, no account is taken of the tumour-specific activation of capecitabine. The third activation step, the conversion of 5'-dFUR to 5-FU preferentially takes place within the tumour, because the responsible enzyme, thymidine phosphorylase, is more present in tumour tissue [1]. This means that the FUTP concentrations in tumour cells could be higher than those measured in PBMCs. On the other hand, it could be argued that PBMCs will probably be exposed to higher 5'dFUR concentrations than solid tumour tissues, which in turn could lead to higher FUTP concentrations in PBMCs than in tumour cells.

Nevertheless, we believe that measurement of the active metabolites in PBMCs will provide useful information. By measuring in cells, the intracellular 'activation 
machinery' is at least to some extent represented. Therefore we hypothesize that these measurements in PBMCs will better reflect the active metabolite concentrations in other tissues (including tumour tissue) than the capecitabine and 5-FU plasma concentrations.

Further research is needed to determine whether there is indeed a correlation between the intracellular 5-FU nucleotide levels measured in PBMCs and clinical response or the occurrence of adverse reactions. Depending on the outcome of this study, PBMCs could serve as a surrogate matrix to give an impression of the cytotoxic metabolite concentrations in other tissues.

These intracellular measurements would be primarily useful to optimize dosing regimens for the total population. How long are the active metabolites present within the cell? What would be a suitable dose regimen (i.e. dose and dose interval) based on these results?

The use of intracellular measurements to optimize the capecitabine dose for individual patients (i.e. intracellular therapeutic drug monitoring; TDM) seems further away. The question is, first of all, whether there is a need for individual dose optimization. Our findings indicate that the intracellular FUTP concentrations show considerable interpatient variation, but is this variation associated with a different clinical outcome, and what about the other two active metabolites? If there is a need for individual dose optimization, a second question is whether intracellular TDM would be feasible. At present the isolation of PBMCs and measurement of the nucleotides is quite laborious, which makes deployment for TDM less practical.

Finally, intracellular measurement of the active metabolite levels might be useful as a predictive marker for treatment response, at least for early stage recognition of nonresponders as a result of a deficient intracellular metabolism. 


\section{REFERENCES}

[1] Miwa M, Ura M, Nishida M, et al. Design of a novel oral fluoropyrimidine carbamate, capecitabine, which generates 5-fluorouracil selectively in tumours by enzymes concentrated in human liver and cancer tissue. Eur J Cancer 1998;34:1274-81.

[2] Schüller J, Cassidy J, Dumont E, et al. Preferential activation of capecitabine in tumor following oral administration to colorectal cancer patients. Cancer Chemother Pharmacol 2000;45:291-297.

[3] Grem J. Mechanisms of Action and Modulation of Fluorouracil. Semin Radiat Oncol 1997;7:249-259.

[4] Álvarez P, Marchal JA, Boulaiz $\mathrm{H}$, et al. 5-Fluorouracil derivatives: a patent review. Expert Opin Ther Pat 2012;22:107-23.

[5] Peters GJ, Laurensse E, Leyva A, et al. Sensitivity of human, murine, and rat cells to 5-fluorouracil and 5'-deoxy-5-fluorouridine in relation to drug-metabolizing enzymes. Cancer Res 1986;46:20-8.

[6] Ciccolini J, Peillard L, Evrard A, et al. Enhanced antitumor activity of 5-fluorouracil in combination with 2'-deoxyinosine in human colorectal cell lines and human colon tumor xenografts. Clin Cancer Res 2000;6:15291535.

[7] Ciccolini J, Peillard L, Aubert C, et al. Monitoring of the intracellular activation of 5 -fluorouracil to deoxyribonucleotides in HT29 human colon cell line: application to modulation of metabolism and cytotoxicity study. Fundam Clin Pharmacol 2000;14:147154.

[8] Kamm YJL, Peters GJ, Hull WE, et al. Correlation between 5-fluorouracil metabolism and treatment response in two variants of $\mathrm{C} 26$ murine colon carcinoma. $\mathrm{Br} \mathrm{J}$ Cancer 2003;89:754-62.

[9] Spears CP, Gustavsson BG, Mitchell MS, et al. Thymidylate synthetase inhibition in malignant tumors and normal liver of patients given intravenous 5-fluorouracil. Cancer Res 1984;44:4144-50.

[10] Spears CP, Gustavsson BG, Berne M, et al. Mechanisms of innate resistance to thymidylate synthase inhibition after 5-fluorouracil. Cancer Res 1988;48:58945900 .
[11] Peters GJ, Lankelma J, Kok RM, et al. Prolonged retention of high concentrations of 5 -fluorouracil in human and murine tumors as compared with plasma. Cancer Chemother Pharmacol 1993;31:269-76.

[12] Moran RG, Spears CP, Heidelberger C. Biochemical determinants of tumor sensitivity to 5-fluorouracil: ultrasensitive methods for the determination of 5-fluoro2'-deoxyuridylate, 2'-deoxyuridylate, and thymidylate synthetase. Proc Natl Acad Sci USA 1979;76:1456-60.

[13] Derissen EJB, Hillebrand MJX, Rosing H, et al. Development of an LC-MS/MS assay for the quantitative determination of the intracellular 5-fluorouracil nucleotides responsible for the anticancer effect of 5-fluorouracil. J Pharm Biomed Anal 2015;110:58-66.

[14] Deenen MJ, Rosing H, Hillebrand MJ, et al. Quantitative determination of capecitabine and its six metabolites in human plasma using liquid chromatography coupled to electrospray tandem mass spectrometry. $J$ Chromatogr B Analyt Technol Biomed Life Sci 2013;913-914:30-40.

[15] Sharma S, Cabana R, Shariatmadar S, et al. Cellular volume and marker expression in human peripheral blood apheresis stem cells. Cytometry A 2008;73:160-167.

[16] Simiele M, D'Avolio A, Baietto L, et al. Evaluation of the mean corpuscular volume of peripheral blood mononuclear cells of HIV patients by a coulter counter to determine intracellular drug concentrations. Antimicrob Agents Chemother 2011;55:2976-2978.

[17] Reigner B, Verweij J, Dirix L, et al. Effect of food on the pharmacokinetics of capecitabine and its metabolites following oral administration in cancer patients. Clin Cancer Res 1998;4:941948.

[18] Reigner B, Blesch K, Weidekamm E. Clinical pharmacokinetics of capecitabine. Clin Pharmacokinet 2001;40:85-104.

[19] Chen P, Liu Z, Liu S, et al. A LC-MS/MS method for the analysis of intracellular nucleoside triphosphate levels. Pharm Res 2009;26:1504-15. 
[20] Cohen S, Megherbi M, Jordheim LP, et al. Simultaneous analysis of eight nucleoside triphosphates in cell lines by liquid chromatography coupled with tandem mass spectrometry. I Chromatogr B Analyt Technol Biomed Life Sci 2009;877:3831-40.

[21] Sobrero AF, Aschele C, Bertino JR. Fluorouracil in colorectal cancer - A tale of two drugs: Implications for biochemical modulation. J Clin Oncol 1997;15:368-381.

[22] Hoff PM, Cassidy J, Schmoll HJ. The evolution of fluoropyrimidine therapy: from intravenous to oral. Oncologist 2001;6 Suppl 4:3-11.

[23] Piedbois P. Efficacy of intravenous continuous infusion of fluorouracil compared with bolus administration in advanced colorectal cancer. J Clin Oncol 1998;16:301-308.

[24] Pogolotti A, Nolan P, Santi D. Methods for the complete analysis of 5-fluorouracil metabolites in cell extracts. Anal Biochem 1981;117:178-86.

[25] Washtien WL, Santi DV. Assay of intracellular free and macromolecular-bound metabolites of 5-fluorodeoxyuridine and 5-fluorouracil. Cancer Res 1979;39:3397-404. 


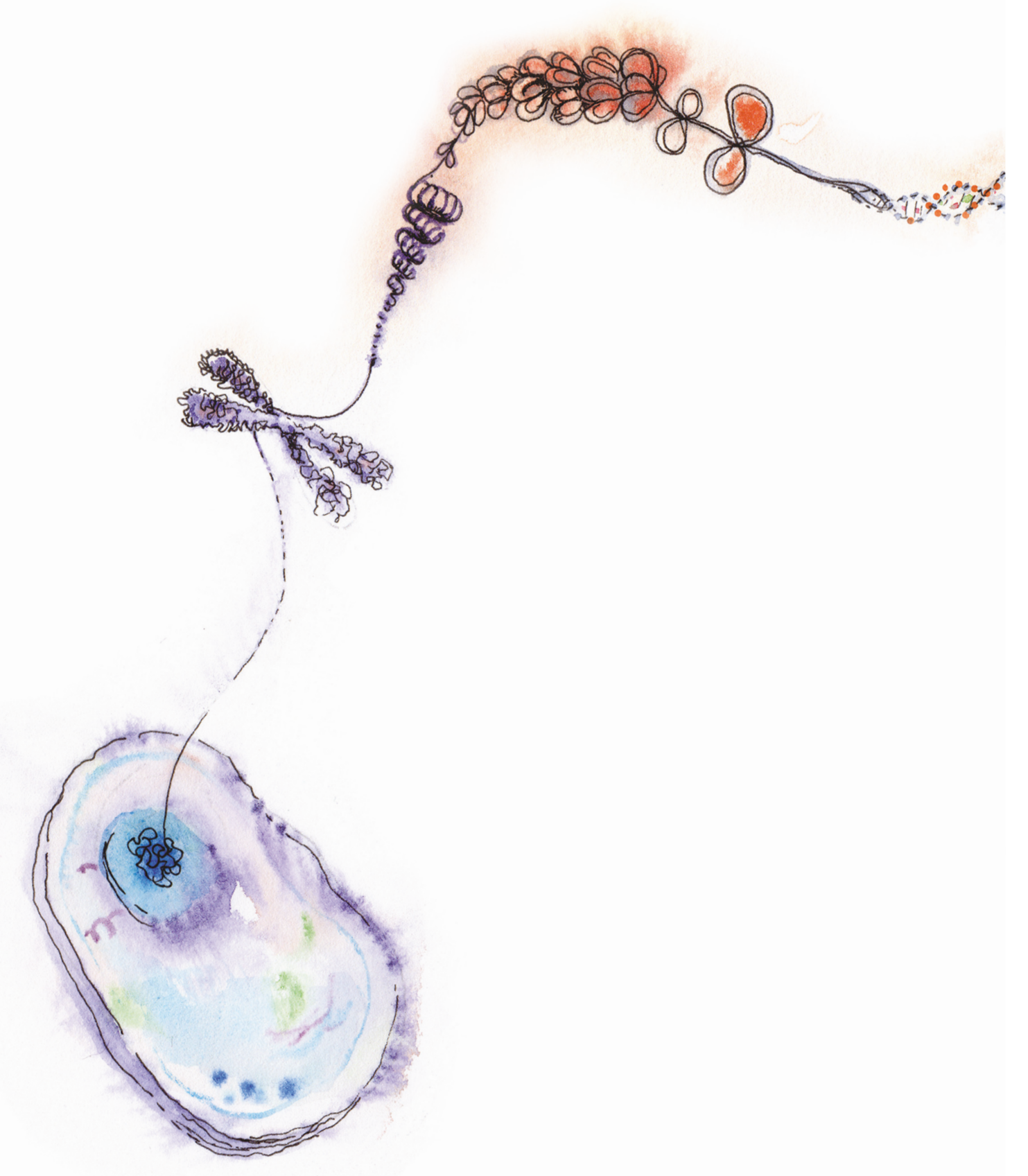


मों

in

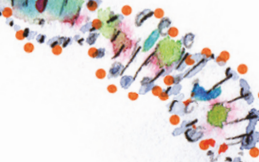

\author{
Gemcitabine
}

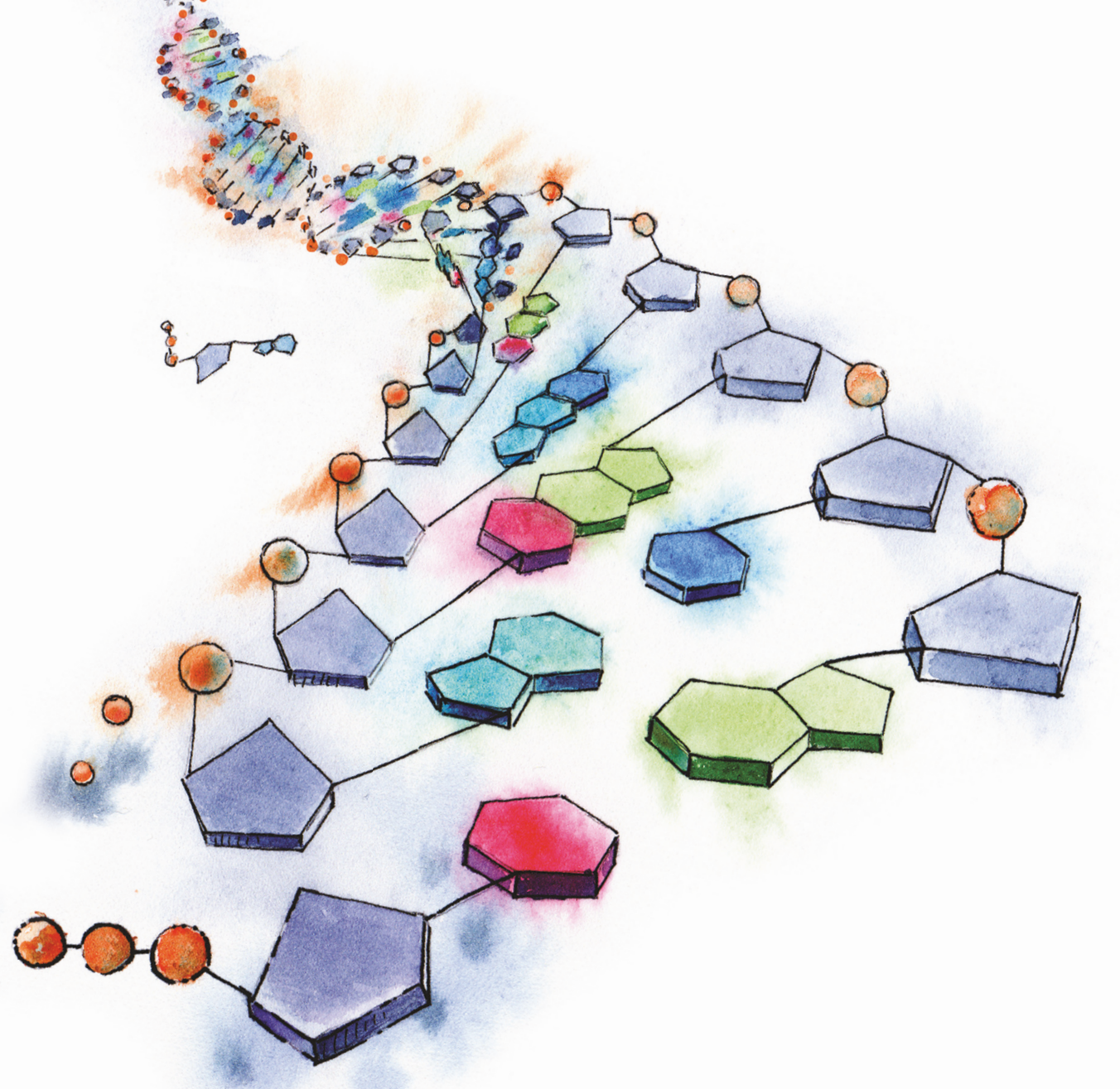




\section{CHAPTER 4.1}

Intracellular pharmacokinetics of gemcitabine, its deaminated metabolite 2',2'-difluorodeoxyuridine and their nucleotides

British Journal of Clinical Pharmacology 2018;84:1279-1289.

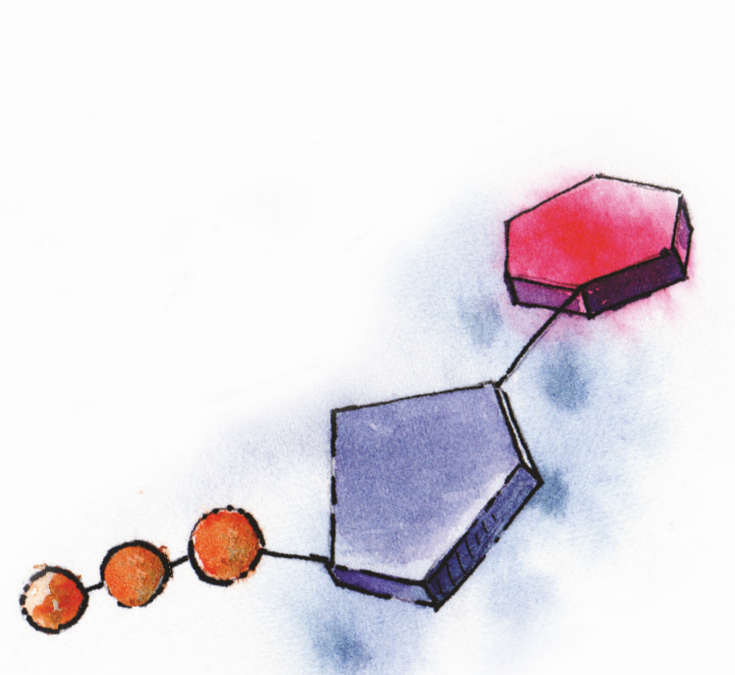

Ellen J.B. Derissen Alwin D.R. Huitema Hilde Rosing Jan H.M. Schellens Jos H. Beijnen 


\section{ABSTRACT}

AIMS Gemcitabine (2',2'-difluoro-2'-deoxycytidine, dFdC) is a prodrug that has to be phosphorylated within the tumour cell to become active. Intracellularly formed gemcitabine diphosphate (dFdCDP) and triphosphate (dFdCTP) are considered responsible for the antineoplastic effects of gemcitabine. However, a major part of gemcitabine is converted into 2',2'-difluoro-2'-deoxyuridine (dFdU) by deamination. In the cell, dFdU can also be phosphorylated to its monophosphate (dFdUMP), diphosphate (dFdUDP) and triphosphate (dFdUTP). In vitro data suggest that these dFdU nucleotides might also contribute to the antitumour effects, although, little is known about their intracellular pharmacokinetics (PK). Therefore, the objective of the present study was to gain insight into the intracellular PK of all $\mathrm{dFdC}$ and $\mathrm{dFdU}$ nucleotides formed during gemcitabine treatment.

METHODS Peripheral blood mononuclear cell (PBMC) samples were collected from 38 patients receiving gemcitabine, at multiple time points after infusion. Gemcitabine, dFdU and their nucleotides were quantified in PBMCs. In addition, gemcitabine and dFdU plasma concentrations were monitored. The individual PK parameters in plasma and in PBMCs were determined.

RESULTS Both in plasma and in PBMCs, dFdU was present in higher concentrations than gemcitabine [mean intracellular area under the concentration-time curve from time zero to $24 \mathrm{~h}\left(\mathrm{AUC}_{0-24 \mathrm{~h}}\right) 1650 \mathrm{vs} .95 \mu \mathrm{M}^{*} \mathrm{~h}$ ]. However, the dFdUMP, dFdUDP and dFdUTP concentrations in PBMCs were much lower than the $d F d C D P$ and $d F d C T P$ concentrations. The mean $A \mathrm{C}_{0-24 \mathrm{~h}}$ for dFdUTP was $312 \mu \mathrm{M}^{*} \mathrm{~h}$ vs. $2640 \mu \mathrm{M}^{*} \mathrm{~h}$ for dFdCTP.

CONCLUSIONS This study provides the first complete picture of all nucleotides that are formed intracellularly during gemcitabine treatment. Low intracellular dFdU nucleotide concentrations were found, which calls into question the relevance of these nucleotides for the cytotoxic effects of gemcitabine.

Keywords: pharmacokinetics; gemcitabine; nucleotides; 2',2'-difluorodeoxycytidine triphosphate (dFdCTP); 2',2'-difluorodeoxyuridine triphosphate (dFdUTP), peripheral blood mononuclear cells (PBMCs). 


\section{INTRODUCTION}

Gemcitabine (2',2'-difluoro-2'-deoxycytidine, dFdC) is an important anticancer drug in the treatment of a variety of solid tumours, including advanced pancreatic, bladder, breast, ovarian, and nonsmall cell lung cancer. It is a deoxycytidine analogue in which the two hydrogen atoms at the 2'-position of the deoxyribose moiety are replaced by fluorine.

To become active, gemcitabine has to enter the tumour cell, where it has to be activated by phosphorylation. The intracellular metabolism of gemcitabine is illustrated in Fig. 1. Cellular uptake of this agent is largely mediated by human equilibrative nucleoside transporter (hENT) 1 and, to a lesser extent, also by hENT2 and the human concentrative nucleoside transporters (hCNT) 1 and 3 [1,2]. Once inside the cell, gemcitabine is phosphorylated in the cytoplasm by deoxycytidine kinase (dCK) to its monophosphate (dFdCMP) and then by pyrimidine nucleoside monophosphate kinase (UMP-CMP kinase) to gemcitabine diphosphate (dFdCDP). The enzyme responsible for the final phosphorylation step, the phosphorylation of dFdCDP to gemcitabine triphosphate (dFdCTP) is unclear, although nucleoside diphosphate kinase may carry out this role [3].

Intracellularly formed dFdCDP and dFdCTP are considered responsible for the cytotoxic effects of gemcitabine. dFdCTP competes with deoxycytidine triphosphate (dCTP) for incorporation into DNA. When dFdCTP is incorporated into DNA, this interferes with the DNA synthesis and triggers apoptosis [4-6]. An additional mechanism of action of gemcitabine is self-potentiation. dFdCDP inhibits the enzyme ribonucleotide reductase which catalyses the reduction of ribonucleotides to deoxyribonucleotides [7]. By inhibiting this enzyme, the intracellular deoxyribonucleotide triphosphate (dNTP) pool is depleted. This increases the dFdCTP / dCTP ratio, which favours the incorporation of dFdCTP into the DNA. As dCK activity is also regulated by dCTP, reducing the dNTP pool also promotes gemcitabine phosphorylation [8].

A major form of elimination of gemcitabine is deamination to 2',2'-difluoro-2'deoxyuridine ( $\mathrm{dFdU}$ ) by cytidine deaminase (Fig. 1). This deamination takes place in the liver, in the blood compartment, and within normal and tumour cells $[9,10]$. dFdU can also be taken up by cells - for instance, by hCNT1 [11]. 


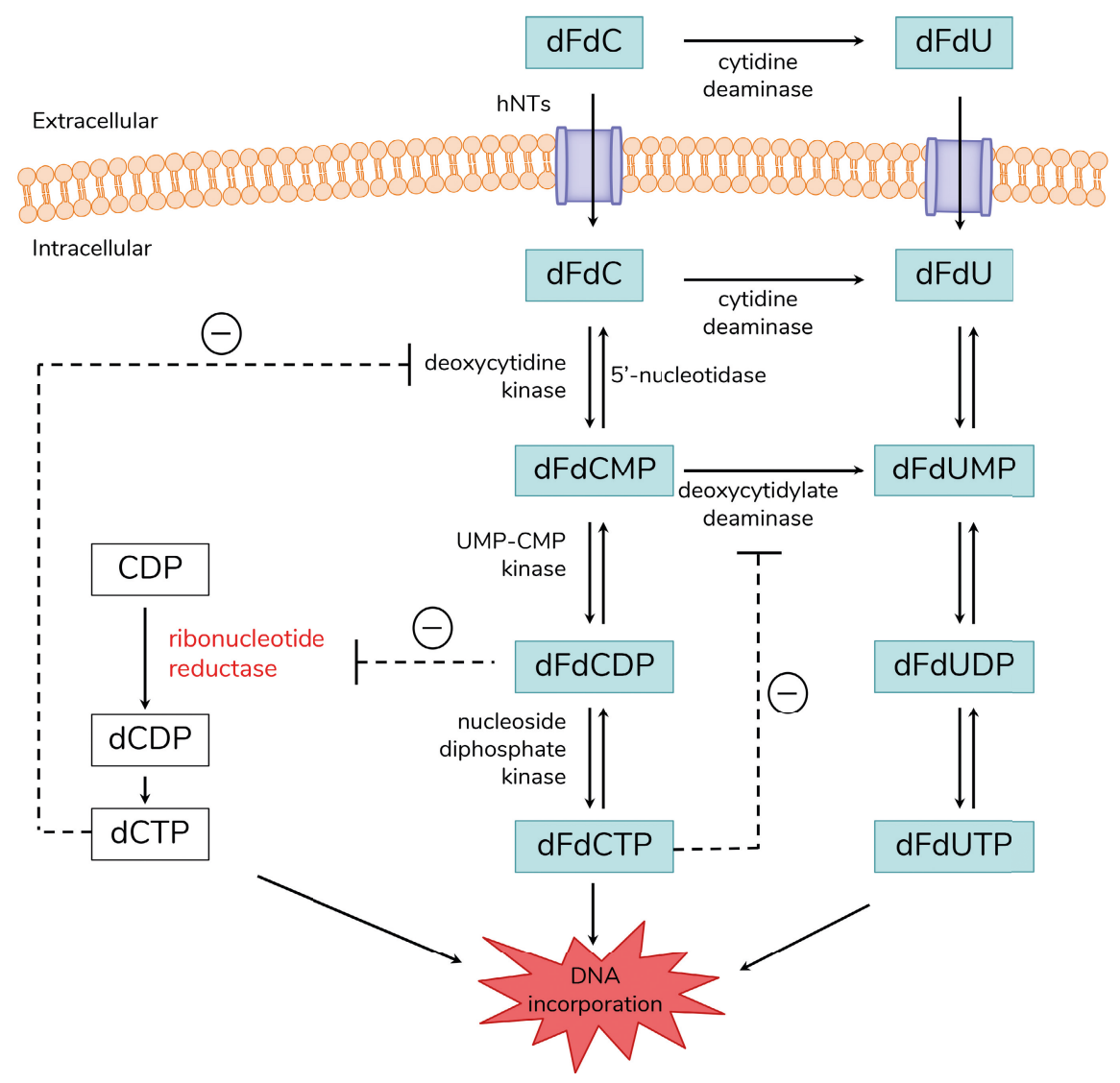

Figure 1. Gemcitabine metabolism and mechanisms of action

Abbreviations: $d F d C, \quad 2^{\prime}, 2^{\prime}$-difluorodeoxycytidine (gemcitabine); dFdCMP, 2',2'-difluorodeoxycytidine 5'-monophosphate; dFdCDP, 2',2'-difluorodeoxycytidine 5'-diphosphate; dFdCTP, 2',2'-difluorodeoxycytidine 5'-triphosphate; $d F d U$, 2',2'-difluorodeoxyuridine; $d F d U M P, 2$ ', 2'-difluorodeoxyuridine 5'-monophosphate; $d F d U D P$, 2',2'-difluorodeoxyuridine 5'-diphosphate; $d F d U T P$, 2',2'-difluorodeoxyuridine 5'-triphosphate; $d C M P$, deoxycytidine monophosphate; $d C D P$, deoxycytidine diphosphate; $d C T P$, deoxycytidine triphosphate; $h N T s$, human nucleoside transporters; UMP-CMP kinase, pyrimidine nucleoside monophosphate kinase.

In the cell, dFdU can be phosphorylated to its monophosphate (dFdUMP), diphosphate (dFdUDP) and triphosphate (dFdUTP) [11]. Alternatively, dFdCMP can be deaminated to dFdUMP, by deoxycytidylate deaminase, and then further phosphorylated $[9,10]$. In vitro studies have demonstrated that dFdUTP is incorporated into both DNA and RNA, and that the extent of incorporation is correlated with the cytotoxicity of dFdU [11]. This suggests that dFdUTP 
incorporation might also contribute to the cytotoxicity of gemcitabine. In addition, recent studies have indicated that dFdUMP, which is structurally similar to deoxyuridine monophosphate (dUMP), inhibits the enzyme thymidylate synthase in tumour cell lines. This might also contribute to the cytotoxic effect of gemcitabine [12].

Measurement of the intracellular concentrations of the active metabolites during gemcitabine treatment provides useful information that might help to optimize dosing regimens for gemcitabine. Therefore, numerous studies have examined the intracellular pharmacokinetics (PK) of dFdCTP in patients treated with gemcitabine [13-21]. However, much less is known about the intracellular PK of dFdCDP, which also contributes to the activity of gemcitabine. In addition, very little is known about the intracellular PK of the dFdU nucleotides (i.e. dFdUMP, dFdUDP and dFdUTP) during gemcitabine treatment, although in vitro data suggest that these $\mathrm{dFdU}$ nucleotides might also contribute to the cytotoxic effect of gemcitabine [11]. The objective of the present study was to gain insight into the intracellular concentration-time course of all $\mathrm{dFdC}$ and $\mathrm{dFdU}$ nucleotides during treatment with gemcitabine. To this end, the intracellular concentrations of gemcitabine, dFdU and all nucleotides that could be expected, i.e. dFdCMP, dFdCDP, dFdCTP, dFdUMP, dFdUDP and dFdUTP, were monitored in peripheral blood mononuclear cells (PBMCs) obtained at several time points during and after gemcitabine treatment.

\section{METHODS}

\section{Study design and treatment schedule}

The intracellular PK of gemcitabine, $\mathrm{dFdU}$ and their nucleotides was examined in two patient cohorts. Cohort A consisted of 12 female patients who participated in a phase I study in advanced breast cancer [22], exploring the safety, PK profile and the preliminary antitumour activity of the combination of gemcitabine and lapatinib. Gemcitabine was given as a 30 -min intravenous infusion at a dose of $1000 \mathrm{mg} / \mathrm{m}^{2}$ on days 1,8 and 15 of a 28-day treatment cycle. Patients received lapatinib once daily according to a dose escalation schedule. PBMC samples were collected on day 1 of the first treatment cycle prior to gemcitabine infusion, 
at the end of the infusion and $2 \mathrm{~h}$ and $24 \mathrm{~h}$ after the start of the infusion. On the same day, plasma samples were collected to monitor gemcitabine and dFdU plasma concentrations prior to, at the end of, and at 1, 2, 4, 8 and $24 \mathrm{~h}$ after start of the infusion.

Cohort B consisted of 26 patients with advanced solid tumours who were participating in a phase I study investigating the safety, PK and preliminary antitumour activity of the combination of gemcitabine, sorafenib and carboplatin. Gemcitabine was given as a 30-min intravenous infusion of $500 \mathrm{mg} / \mathrm{m}^{2}$ on days 1 and 8 of a 21-day treatment cycle. Patients received sorafenib once or twice daily according to a dose escalation schedule. Carboplatin (AUC 2 or 3 $\mathrm{mg}^{*} \mathrm{~min} / \mathrm{ml}$ ) was administered as a 30-min intravenous infusion on day 1 of every cycle. The gemcitabine infusion was started 90 minutes after the start of the carboplatin infusion. PBMC samples were collected on day 1 of the first treatment cycle prior to the infusion, at the end of the infusion, and $2 \mathrm{~h}$ and 22 $\mathrm{h}$ after the start of the infusion. Plasma samples were collected prior to, at the end of and at 2, 4, 6 and $22 \mathrm{~h}$ after the start of the gemcitabine infusion.

The studies were approved by the medical ethics committee of our Institute and were conducted in accordance with the Declaration of Helsinki. All patients provided written informed consent before enrolment.

\section{Quantification of gemcitabine and dFdU in plasma}

To monitor the gemcitabine and dFdU plasma concentrations, $3 \mathrm{~mL}$ of blood was collected at the defined time points. Samples were collected in heparinized tubes containing $500 \mu \mathrm{g}$ of the cytidine deaminase inhibitor tetrahydrouridine (Calbiochem, La Jolla, CA, USA) and were immediately centrifuged at $4{ }^{\circ} \mathrm{C}$ for $5 \mathrm{~min}$ at $1500 \times \mathrm{g}$. Plasma was collected and stored at $-20^{\circ} \mathrm{C}$ until analysis. Gemcitabine and dFdU were quantified in plasma by a validated LC-MS/MS assay, as previously described by our group [23] with some minor adjustments. In brief, ${ }^{13} \mathrm{C}^{15} \mathrm{~N}_{2}$-labelled isotopes of the analytes were used as internal standards and added to $200 \mu \mathrm{L}$ of plasma. A solid-phase extraction (SPE) was performed using OASIS HLB 30 mg SPE cartridges (Waters Corporation, Milford, MA, USA). The SPE cartridges were conditioned with $500 \mu \mathrm{L}$ methanol and 500 $\mu \mathrm{L}$ water. The plasma sample was then loaded onto the cartridge, followed 
by $500 \mu \mathrm{L}$ water. After drying the cartridge for 2 min with air, the analytes were eluted with $400 \mu \mathrm{L}$ methanol. Methanol was evaporated under a gentle stream of nitrogen at $40{ }^{\circ} \mathrm{C}$ and the residue was redissolved in $100 \mu \mathrm{L}$ of reconstitution solvent (1 mM ammonium acetate ( $\mathrm{pH}$ 6.8) / acetonitrile (97:3, v/v)). Chromatographic separations were performed using a Synergi Hydro-RP column (150 mm x $4.6 \mathrm{~mm}$ ID, particle size $5 \mu \mathrm{m}$; Phenomenex, Torrance, CA, USA) kept at $30{ }^{\circ} \mathrm{C}$. The mobile phase consisted of $1 \mathrm{mM}$ ammonium acetate ( $\mathrm{pH}$ 6.8) / acetonitrile (94:6, v/v) and was delivered isocratically at a flow rate of $0.2 \mathrm{~mL} / \mathrm{min}$. Detection of the analytes was performed on an API3000 triple quadrupole mass spectrometer equipped with an electrospray ionization probe (Sciex, Framingham, MA, USA). Gemcitabine was detected in the positive ion mode and $\mathrm{dFdU}$ in negative ion mode. The validated concentration ranges in plasma were $0.5-1000 \mathrm{ng} / \mathrm{mL}$ (1.90-3799 nM) for gemcitabine and 50-10000 $\mathrm{ng} / \mathrm{mL}$ (189-37853 $\mathrm{nM}$ ) for dFdU. The accuracies for gemcitabine and dFdU in plasma were within $\pm 16.3 \%$ at the lower limit of quantification (LLOQ) and within $\pm 10.7 \%$ at the other levels tested. The precisions were $<12.2 \%$ at all concentration levels.

\section{Quantification of gemcitabine, dFdU and their nucleotides in PBMCs}

Previous studies performed by our group demonstrated that, if samples were processed on ice-water, the intracellular $\mathrm{dFdC}$ and $\mathrm{dFdU}$ nucleotide concentrations in PBMCs remained stable for at least 3 hours and were not influenced by the addition of a cytidine deaminase inhibitor (tetrahydrouridine) or deoxycytidylate deaminase inhibitor (zebularine) [24]. Based on these data, PBMCs were isolated and processed on ice-water, as described previously [25], without addition of an enzyme inhibitor. In brief, $15 \mathrm{~mL}$ of blood was collected in heparinized tubes and PBMCs were isolated immediately using cold Ficoll-Paque PLUS density gradient (GE Healthcare, Pittsburgh, PA, USA). The collected PBMCs were resuspended in $100 \mu \mathrm{L}$ cold phosphate-buffered saline (PBS), resulting in a homogeneous cell suspension with a total volume of approximately $130 \mu \mathrm{L}$. A $10 \mu \mathrm{L}$ aliquot of this cell suspension was used for the analysis of protein concentrations using the Bio-Rad Protein Assay [26], which was corrected for interference by residual haemoglobin as previously described by our group [27]. A $100 \mu \mathrm{L}$ aliquot of the cell suspension was 
used for the quantification of gemcitabine, dFdU and their monophosphates, diphosphates and triphosphates. To this end, cells were lysed by the addition of $100 \mu \mathrm{L} 1.2 \mathrm{M}$ perchloric acid $\left(\mathrm{HClO}_{4}\right)$ followed by extensive vortex mixing. After centrifugation ( $5 \mathrm{~min}, 1500 \times \mathrm{g}$, at $4^{\circ} \mathrm{C}$ ), the supernatant (PBMC lysate) was collected and stored at $-70{ }^{\circ} \mathrm{C}$ until analysis. Directly prior to LC-MS/MS analysis, the PBMC lysates were spiked with internal standard: a mixture of ${ }^{13} \mathrm{C}^{15} \mathrm{~N}_{2}$-labelled gemcitabine and dFdU nucleotides, which were synthesized in-house [24].

The concentrations of gemcitabine, dFdCMP, dFdCDP, dFdCTP, dFdU, dFdUMP, dFdUDP and dFdUTP in the PBMC Iysates were determined using the validated simultaneous LC-MS/MS assay previously described by our group [24], with some minor adjustments. In brief, chromatographic separation of all eight compounds was obtained using a porous graphitic carbon (Hypercarb) column (100 mm × $2.1 \mathrm{~mm}$ ID, $5 \mu \mathrm{m}$ particles, Thermo Fisher Scientific Inc., Waltham, MA, USA) kept at $45^{\circ} \mathrm{C}$. Elution was performed using a gradient from 0 to 25 $\mathrm{mM}$ ammonium bicarbonate in acetonitrile-water (15:85, v/v). As previously described, the chromatographic separation was only reproducible when the redox state of the porous graphitic carbon was carefully maintained and the column was properly cleaned $[24,28]$. Therefore, before each analytical run the column was treated for 30 minutes with preconditioning buffer (consisting of 1 $\mathrm{mM}$ ammonium acetate in acetonitrile/ water $(15: 85, \mathrm{v} / \mathrm{v})$ with $0.05 \%$ hydrogen peroxide and with the $\mathrm{pH}$ adjusted to 4 with glacial acetic acid); between each analytical injection $100 \mu \mathrm{L}$ diluted formic acid (10\% v/v, in water) was injected, and after each analytical run the column was back-flushed with about 20 column volumes tetrahydrofuran [24].

Detection was performed on an API4000 triple quadrupole mass spectrometer equipped with an electrospray ionization probe operating in the positive ionization mode (Sciex). Calibration ranges in PBMC lysate were 2.91-289.1 nM for dFdC; 22.1-2197 nM for dFdCMP; 46.9-4655 nM for dFdCDP; 39.23888 nM for dFdCTP; 49.0-4864 nM for dFdU; 20.8-2063 nM for dFdUMP; 48.9-4847 nM for dFdUDP and 46.7-4630 nM for dFdUTP. Accuracies were within $\pm 19.4 \%$ at the lower limits of quantification (LLOQ) and within $\pm 14.4 \%$ at the other levels tested. The precisions were $<19.7 \%$ at the LLOQ and $<14.9 \%$ at the other levels tested. 
The analytical results, expressed as $\mathrm{nM}$ in PBMC lysate, were multiplied with the lysate sample volume to obtain the absolute nucleoside and nucleotide amounts in a sample. These amounts were then divided by the number of cells in the sample to obtain the nucleoside and nucleotide amounts per million PBMCs. Instead of performing a direct cell count, which requires immediate analysis of each sample, the number of cells was derived from the amount of protein in the sample, as samples could be stored for longer periods prior to analysis of the protein concentrations. In addition, we assessed the correlation between the cellular protein content and the cell number. To this end, a cell count was performed for 27 PBMC samples prior to cell lysis. The mean PBMC concentration in the cell suspensions in PBS was $53 \times 10^{6} \mathrm{PBMCs} / \mathrm{mL}$ [coefficient of variation $(\mathrm{CV})=81 \%$ ]. The mean amount of protein in the cell suspensions (corrected for haemoglobin) was $6.6 \mathrm{mg} / \mathrm{mL}(\mathrm{CV}=60 \%)$. Thus, the samples contained on average $0.12 \mathrm{mg}$ protein/ $10^{6} \mathrm{PBMCs}$.

In order to compare the intracellular concentrations with the plasma concentrations, the intracellular concentrations were also converted to the nucleoside and nucleotide amounts per volume unit $(\mu \mathrm{M})$. To this end, the number of PBMCs in each sample was derived from the amount of protein and an average cell volume of $282.9 \mathrm{fL}$ was assumed (as determined by Simiele et al. [29]).

\section{PK and statistical analysis}

The individual PK parameters in plasma and in PBMCs were derived using noncompartmental analysis using validated scripts in the software package $R$ (version 3.1.2). Sufficient plasma samples were collected from each patient, to determine the plasma half-lives $\left(t_{1 / 2}\right)$ of $d F d C$ and $d F d U$ and to extrapolate the areas under the plasma concentration - time curves to infinity $\left(A \cup C_{\text {inf }}\right)$. The terminal plasma $t_{1 / 2}$ of $\mathrm{dFdC}$ and $\mathrm{dFdU}$ were calculated based on the last three data points.

The number of PBMC samples collected per patient was limited to minimize the burden of blood sampling (as a larger blood volume of $15 \mathrm{~mL}$ was required per time point, to isolate sufficient PBMCs) and because of the practical feasibility (each sample had to be processed immediately after collection owing to limited 
stability of the analytes). As only four PBMC samples were collected per patient, it was not possible to determine the intracellular $t_{1 / 2}$. Instead, the percentage of the maximum observed concentration $\left(C_{\text {max }}\right)$ that was still present $22 \mathrm{~h}$ or $24 \mathrm{~h}$ after gemcitabine infusion was reported. Despite the limited number of PBMC sample times, the intracellular AUC from time zero up to the last sample time $\left(\mathrm{AUC}_{0-\mathrm{t}}\right)$ was calculated.

\section{RESULTS}

\section{Plasma PK of gemcitabine and dFdU}

The mean plasma concentration-time curves for gemcitabine and dFdU are shown in Fig. 2 and the results of the noncompartmental PK analysis in plasma are shown in Table 1. As expected, gemcitabine plasma concentrations were highest at the end of the infusion. The highest dFdU plasma concentration was also measured at this time in the majority of patients, indicating that gemcitabine is converted rapidly to dFdU.

Patients in cohort $A$ received gemcitabine doses twice as high as patients in cohort B. However, the maximum observed gemcitabine plasma concentrations and AUCs were almost four times higher for cohort $A$ than for cohort B. For the dFdU plasma concentrations, by contrast, a dose-proportional increase was observed for cohort A compared with cohort B. We had no clear explanation for the differences found between the two patient cohorts. One possibility was that the comedication had had some influence. However, previous combination studies with lapatinib [22,30], sorafenib [31] and carboplatin [32] showed no clinically relevant influence of concomitant use of these drugs on the plasma PK of gemcitabine.

In all patients, the dFdU levels measured in plasma were substantially higher than the gemcitabine levels. In addition, dFdU remained in the plasma for longer. Two hours after the start of the infusion, gemcitabine was only present at very low concentrations, and 24 hours after the start of the infusion gemcitabine had almost completely disappeared, whereas the dFdU concentrations were, 

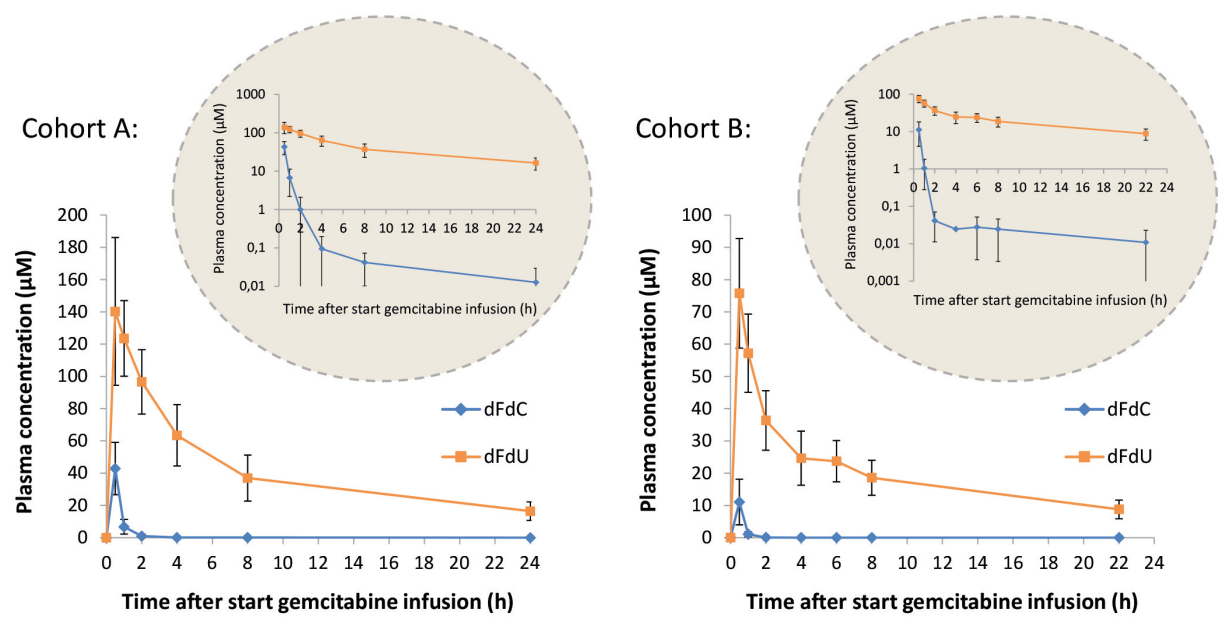

Figure 2. Plasma concentrations of gemcitabine (dFdC) and 2',2'-difluoro-2'-deoxyuridine ( $\mathrm{dFdU}$ ) for patient cohorts $A$ and $B$, receiving gemcitabine $1000 \mathrm{mg} / \mathrm{m}^{2}$ and $500 \mathrm{mg} / \mathrm{m}^{2}$, respectively. The data are shown as mean values (symbols) with standard deviations (error bars). Inset: semi-logarithmic presentation.

on average, still $12 \%$ of the $\mathrm{C}_{\max }$. The $\mathrm{dFdU}$ plasma exposure was, therefore, much higher than the gemcitabine plasma exposure in both patient cohorts.

Although gemcitabine concentrations were low within $2 \mathrm{~h}$ after the infusion, in most patients very low gemcitabine plasma concentrations $(<0.06 \mu \mathrm{M})$ were measurable up to 24 hours after infusion. This is more apparent when the data are displayed on a semi-logarithmic scale (Fig. 2 inset). Plasma concentrations of $\mathrm{dFdU}$ peaked at the end of infusion, followed by a rapid distribution phase and a longer elimination phase. The plasma $t_{1 / 2}$ values reported here refer to the terminal elimination phase and were based on the last three sampling time points.

\section{Intracellular PK of gemcitabine, dFdU and their nucleotides}

The mean intracellular concentration-time curves of gemcitabine, dFdU and their nucleotides are shown in Fig. 3, and the results of the noncompartmental analysis of the intracellular PK data are presented in Table 2. The highest intracellular gemcitabine and dFdU concentrations were measured the end of the infusion. The intracellular gemcitabine and dFdU concentrations increased 
Table 1. Summary statistics for the pharmacokinetic parameters of gemcitabine (dFdC) and its metabolite $\mathrm{dFdU}$ in plasma. The data are shown as mean values [range].

\begin{tabular}{|c|c|c|}
\hline & $\mathrm{dFdC}$ & dFdU \\
\hline \multicolumn{3}{|l|}{ Cohort A (receiving gemcitabine 1000 mg/m²) } \\
\hline $\mathrm{n}$ & 12 & 12 \\
\hline$C_{\max }(\mu M)$ & $42.8[16.7-81.7]$ & 147 [99.9-224] \\
\hline$t_{\max }(h)$ & $0.5[0.5-0.5]$ & $0.8[0.5-2]$ \\
\hline$t_{1 / 2, \beta}(h)$ & $7.32[3.43-19.5]$ & $11.2[8.65-13.2]$ \\
\hline $\mathrm{Cl}\left(\mathrm{L} / \mathrm{h} / \mathrm{m}^{2}\right)$ & $153[66.7-341]$ & - \\
\hline Percentage of $\mathrm{C}_{\max }$ still present after $24 \mathrm{~h}(\%)$ & $0.027[<L L O Q-0.14]$ & $12[6.2-24]$ \\
\hline$A \cup C_{0-24 h}(\mu M \bullet h)$ & $28.8[11.1-56.9]$ & 999 [673-1595] \\
\hline$A \cup C_{0-\text { inf }}(\mu M \bullet h)$ & $28.9[11.1-57.0]$ & $1265[869-2010]$ \\
\hline \multicolumn{3}{|l|}{ Cohort B (receiving gemcitabine 500 mg/m²) } \\
\hline$n$ & 26 & 26 \\
\hline$C_{\max }(\mu M)$ & $11.1[0.802-29.3]$ & $75.8[43.5-115]$ \\
\hline$t_{\max }(h)$ & $0.5[0.5-0.5]$ & $0.52[0.5-1]$ \\
\hline$t_{1 / 2, \beta}(h)$ & $10.5[4.16-42.8]$ & $13.9[8.78-24.5]$ \\
\hline $\mathrm{Cl}\left(\mathrm{L} / \mathrm{h} / \mathrm{m}^{2}\right)$ & 324 [109-873] & - \\
\hline Percentage of $\mathrm{C}_{\max }$ still present after $22 \mathrm{~h} \mathrm{( \% )}$ & 0.089 [LLOQ-0.39] & $12[5.6-22]$ \\
\hline$A \cup C_{0-22 h}(\mu M \bullet h)$ & $6.75[0.535-17.3]$ & 478 [289-748] \\
\hline$A \cup C_{0 \text {-inf }}(\mu M \bullet h)$ & 7.89 [2.18-17.5] & 662 [364-1060] \\
\hline
\end{tabular}

Abbreviations: $A \cup C_{0-t}$, the area under the concentration-time curve from time zero to the time point of the last quantifiable data point; $A \cup C_{\text {inf }}$, the area under the concentration-time curve extrapolated to infinity, using the terminal elimination constant; $C$, plasma clearance; $C_{\max }$, the maximum observed concentration; $L L O Q$, lower limit of quantification; $n$, the number of patients for whom pharmacokinetics were evaluable; $t_{1 / 2, \beta}$, terminal elimination half-life; $t_{\max }$, the time to reach the maximum observed concentration.

dose-proportionately: the $\mathrm{C}_{\max }$ for gemcitabine and $\mathrm{dFdU}$ were twice as high in cohort $A$ as in cohort $B$. However, the mean intracellular AUCs of gemcitabine and $\mathrm{dFdU}$ were 4 and 3 times higher, respectively, in cohort $A$ than in cohort $B$.

The gemcitabine and dFdU levels measured in PBMCs were both higher than the levels measured in plasma. The $C_{\max }$ values for gemcitabine and $\mathrm{dFdU}$ in 
Cohort A - receiving gemcitabine $1000 \mathrm{mg} / \mathrm{m}^{2}$
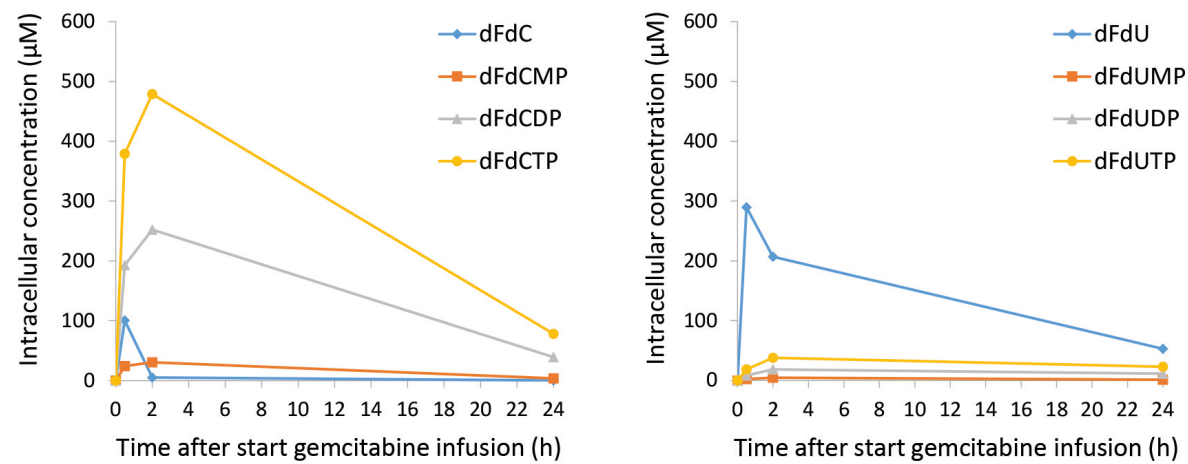

\section{Cohort B - receiving gemcitabine $500 \mathrm{mg} / \mathrm{m}^{2}$}
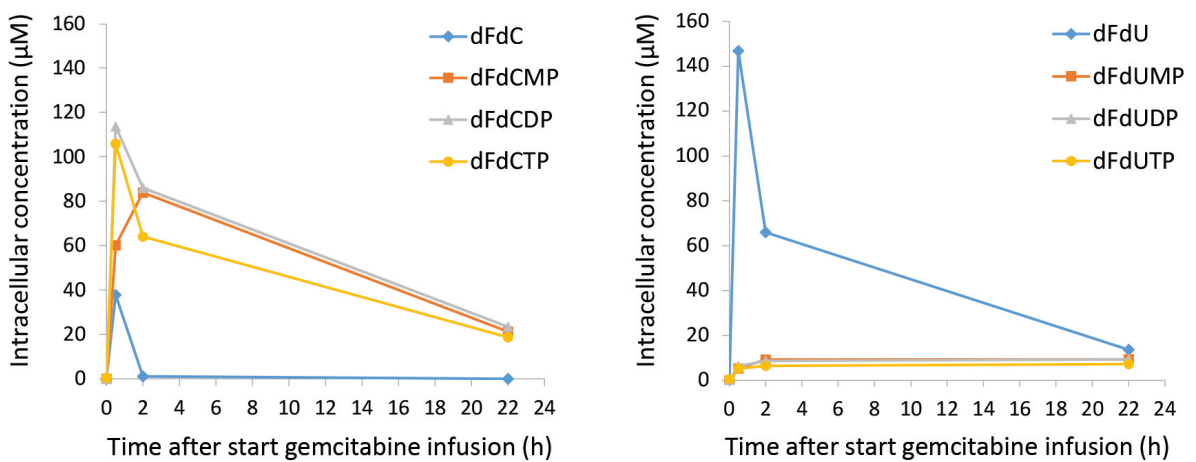

Figure 3. Mean intracellular concentrations of gemcitabine, $\mathrm{dFdU}$ and their nucleotides in PBMCs.

Abbreviations: $d F d C, \quad 2^{\prime}, 2^{\prime}$-difluorodeoxycytidine (gemcitabine); dFdCMP, 2',2'-difluorodeoxycytidine 5'-monophosphate; dFdCDP, 2',2'-difluorodeoxycytidine 5'-diphosphate; dFdCTP, 2',2'-difluorodeoxycytidine 5'-triphosphate; dFdU, 2', 2'-difluorodeoxyuridine; $d F d U M P, 2^{\prime}, 2^{\prime}$-difluorodeoxyuridine 5'-monophosphate; dFdUDP, 2',2'-difluorodeoxyuridine 5'-diphosphate; dFdUTP 2',2'-difluorodeoxyuridine 5'-triphosphate; PBMCs, peripheral blood mononuclear cells.

PBMCs were, on average, 2-3 times higher than the $C_{\max }$ values measured in plasma.

In PBMCs, as well as in plasma, dFdU was present at higher concentrations than gemcitabine. However, the intracellular dFdU nucleotide concentrations (i.e. dFdUMP, dFdUDP and dFdUTP) were relatively low $(<88 \mu \mathrm{M})$ - much lower than the $\mathrm{dFdC}$ nucleotide concentrations that were measured (up to $1060 \mu \mathrm{M}$ ). 


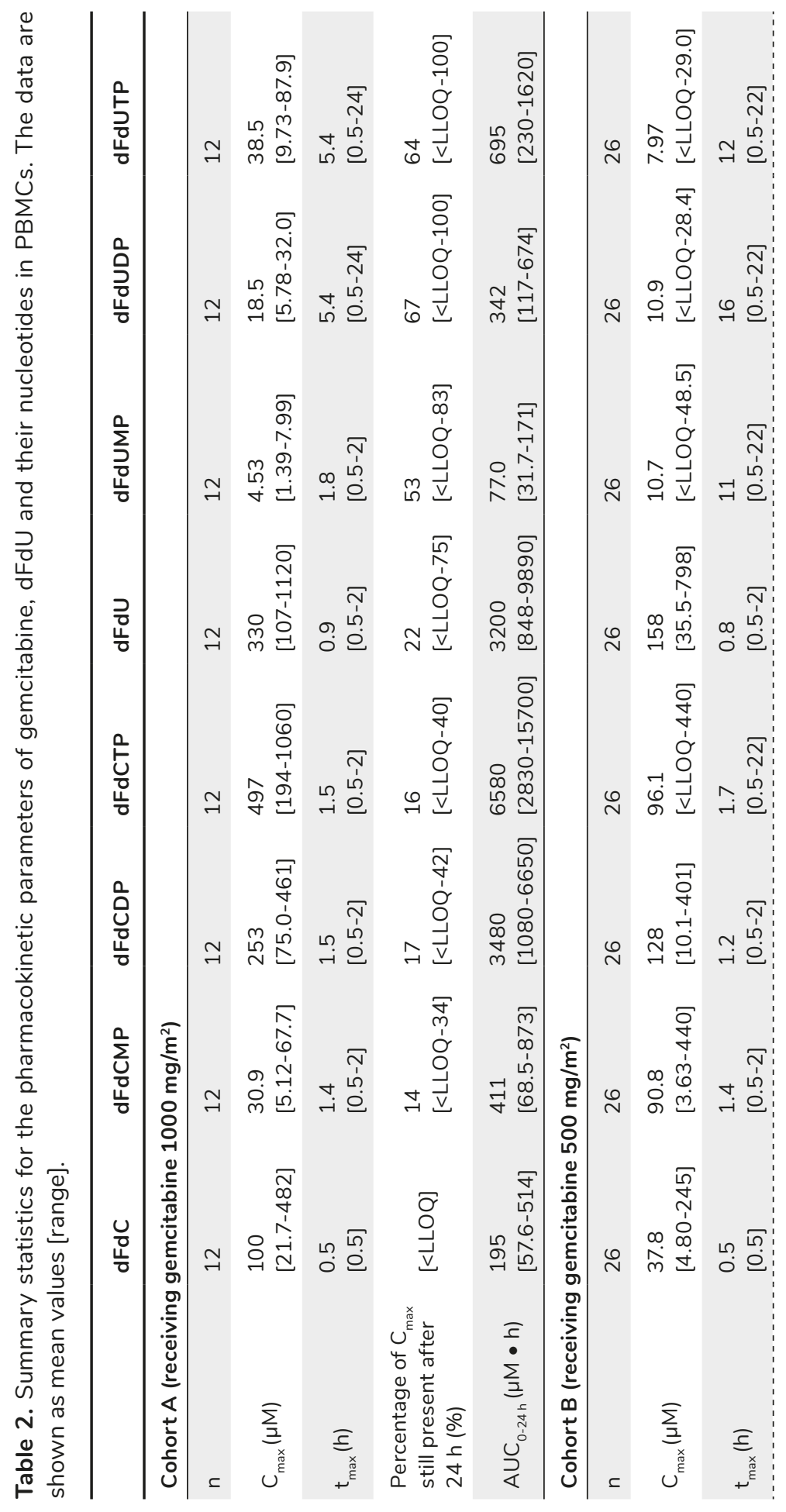




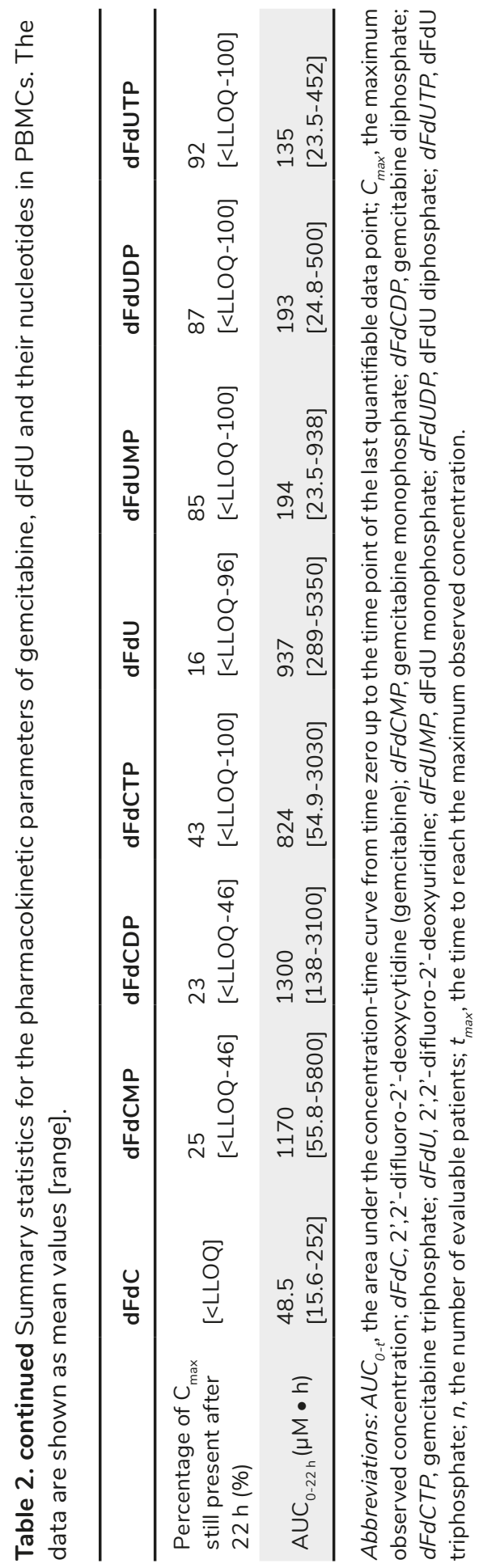


In $8 \%$ of the patients, the dFdU nucleotide concentrations were below the LLOQ of the assay at all time points.

The ratios between the monophosphates, diphosphates and triphosphates differed between the two patient cohorts. In cohort A, dFdCTP showed the highest abundance, followed by dFdCDP. For dFdCMP, dFdUMP, dFdUDP and dFdUTP, only low concentrations were found $(<88 \mu \mathrm{M})$. For cohort $B$, the dFdCMP, dFdCDP and dFdCTP concentrations were approximately equally high. The concentrations of the dFdU nucleotides were also low for this patient cohort $(<49 \mu \mathrm{M})$. We had no clear explanation for the differences between the two patient cohorts. It could not be excluded that the comedication had had an influence on the intracellular nucleotide accumulation. However, to the best of our knowledge, the influences of carboplatin, sorafenib and lapatinib on the intracellular accumulation of dFdCTP have never been studied [21].

Two hours after start of the infusion, very low gemcitabine concentrations were found in PBMCs. However, the $\mathrm{dFdC}$ nucleotides remained present in the cells for much longer. Twenty-four hours after the start of the infusion, substantial intracellular $\mathrm{dFdC}$ nucleotide concentrations were still found in most patients. $\mathrm{dFdU}$ also remained present in the cells for an extended period.

High interindividual variability was observed for the intracellular $\mathrm{C}_{\max }$ and $\mathrm{AUC}$ of gemcitabine, $\mathrm{dFdU}$ and the $\mathrm{dFdC}$ nucleotides. Although some interpatient variability was also seen for the dFdU nucleotide concentrations, these concentrations were systematically low in all patients. 


\section{DISCUSSION}

The plasma PK data found in the present study were in line with previously published data on the plasma PK of gemcitabine and dFdU $[14,19,33,34]$. In addition, the intracellular PK results for dFdCTP were in line with those in previous reports [33-36]. However, data on the intracellular PK of the other $\mathrm{dFdC}$ and $\mathrm{dFdU}$ nucleotides in patients treated with gemcitabine were lacking. The present study provides the first complete picture of the PK of all nucleotides that are formed intracellularly during gemcitabine treatment.

Although dFdU was traditionally believed to be an inactive metabolite, in vitro studies demonstrated that it has cytotoxic activity in tumour cell lines $[11,12,37]$. The half-maximal inhibitory concentration $\left(I_{50}\right)$ for $d F d U$ for gemcitabine-sensitive tumour cell lines is highly variable, but was found to be at least 1000-2000-fold higher than for gemcitabine [11,12,37]. However, Veltkamp et al. showed that the cytotoxicity of dFdU was dependent on the duration of drug exposure [11]. The present study demonstrated that, during gemcitabine treatment, the concentrations of dFdU were well above those of gemcitabine, both in the plasma and in PBMCs. In addition, dFdU had a longer $t_{1 / 2}$ than gemcitabine. Therefore, both in the plasma and in PBMCs, the exposure to $\mathrm{dFdU}$ exceeded the exposure to the parent drug, gemcitabine.

Despite the high intracellular dFdU concentrations that were measured in PBMCs during gemcitabine treatment, the intracellular dFdUMP, dFdUDP and dFdUTP concentrations were much lower than the intracellular dFdCDP and dFdCTP concentrations.

In the present study, PBMCs were used to investigate the intracellular PK of the gemcitabine and dFdU nucleotides. PBMCs are commonly used as a surrogate matrix to study the intracellular PK of nucleoside analogues. They represent the intracellular 'activation machinery' and, unlike tumour cells, can be obtained at multiple time points after drug administration. Nevertheless, it should be kept in mind that the concentrations of nucleotides measured in PBMCs might differ from those that would be found in tumour cells - for instance, because enzymes involved in the synthesis of nucleotides might have a different activity in PBMCs than in tumour cells. As depicted in Fig. 1, dFdU nucleotides can be 
formed via two pathways: I.) by direct phosphorylation of dFdU and II.) through the conversion of dFdCMP to dFdUMP by deoxycytidylate deaminase. Direct phosphorylation of $\mathrm{dFdU}$ is most likely to be mediated by deoxycytidine kinase $(d C K)$ and the mitochondrial thymidine kinase 2 (TK2). However, compared with gemcitabine, $d F d U$ has a much lower affinity for $d C K$ and a higher affinity for TK2 $[38,39]$. The S-phase-specific enzyme thymidine kinase 1 (TK1) was not involved in the phosphorylation of $\mathrm{dFdU}$, as demonstrated by Veltkamp et al. [11]. Unlike TK1, TK2 and $d C K$ are not cell cycle-dependent. However, their activity in PBMCs might be different than in tumour cells. Eriksson et al. showed that the in vitro activity of $d C K$ was higher in (non-activated) PBMCs than in solid tumour tissue [40]. The activity of TK2, by contrast, was lower in (non-activated) PBMCs than in solid tumour tissue [40]. These differences might implicate that $\mathrm{dFdU}$ nucleotide concentrations measured in PBMCs are lower than those that would be found in tumour cells.

The low dFdU nucleotide concentrations might also be related to some of the feedback mechanisms that have previously been described for gemcitabine. For instance, the activity of deoxycytidylate deaminase, the enzyme which is responsible for the conversion of dFdCMP to dFdUMP, is directly inhibited by dFdCTP (Fig. 1). Moreover, lowering of the dCTP pool (an important effect of gemcitabine) is associated with a decrease in the deoxycytidylate deaminase enzyme activity [9]. Previous in vitro studies with arabinosylcytosine (ara-C), another cytidine analogue, demonstrated that deamination via deoxycytidylate deaminase was the predominant pathway in the formation of arabinosyluracil triphosphate (ara-UTP) [41]. Inhibition of deoxycytidylate deaminase suppressed ara-UTP formation from ara- $\mathrm{C}$, whereas inhibition of cytidine deaminase did not perturb the formation of ara-UTP from ara-C [41]. As the intracellular metabolism of gemcitabine resembles the metabolism of ara-C, deamination via deoxycytidylate deaminase might also be the most important pathway for the formation of $\mathrm{dFdU}$ nucleotides. Intracellular feedback mechanisms which inhibit this enzyme might therefore play a substantial role in limiting the degree of deamination of gemcitabine nucleotides.

The question is whether sufficient dFdU is phosphorylated to make a contribution to the cytotoxic effect of gemcitabine. To answer this question, 
the dFdU nucleotide concentrations found in the present study can be compared with the concentrations that have been found in in vitro studies.

Recent cell line studies indicated that dFdUMP, which is structurally similar to deoxyuridine monophosphate (dUMP), might contribute to the cytotoxic effect of gemcitabine by direct inhibition of the enzyme thymidylate synthase [12]. Honeywell et al. demonstrated that, although dFdUMP was a 10,000 fold less potent inhibitor than 5-fluorodeoxyuridine monophosphate (the active metabolite of 5-fluorouracil), inhibition of the enzyme did result in increased dUMP mis-incorporation into the DNA [12]. However, the dFdUMP concentrations measured in PBMCs in the present study were relatively low compared with those that were needed to inhibit thymidylate synthase in the cell line study by Honeywell et al. (inhibitory constant $(\mathrm{Ki})=130 \mu \mathrm{M})$ [12]. This suggests that thymidylate synthase inhibition by dFdUMP does not play a major role, at least not in PBMCs.

Incorporation of dFdUTP into the DNA and RNA, however, might play a role. In the study by Veltkamp et al. incorporation of dFdUTP into both the DNA and the RNA has been found after cells were incubated for $24 \mathrm{~h}$ with relatively low concentrations $(0.5 \mu \mathrm{M})$ of gemcitabine or dFdU [11]. The intracellular dFdUTP concentrations measured in the incubated cells were also relatively low compared with our data in patients. This suggests that dFdU incorporation can also be expected in our patient population. In the study by Veltkamp et al. a strong correlation has been found between the extent of $\mathrm{dFdU}$ incorporation and the cytotoxicity of dFdU [11]. Further studies are warranted to elucidate the importance of the incorporation of dFdU into the DNA for the cytotoxicity of gemcitabine in patients.

It is also interesting to consider the present intracellular PK data in the light of previous clinical reports investigating the relationship between the enzyme activity of cytidine deaminase (CDA) and clinical outcome of gemcitabine therapy. Several studies showed that patients with a low CDA activity displayed more toxicity [42] and better efficacy of gemcitabine-containing therapy $[43,44]$. Conversely, Serdjebi et al. showed that pancreatic cancer patients with high CDA activity were five times more likely to have progressive disease following gemcitabine therapy than patients with normal or low CDA 
activity [45]. The present intracellular PK data support the hypothesis that high dFdU plasma concentrations play a limited role in the cytotoxic effects of gemcitabine therapy, as a result of limited intracellular activation by the formation of $\mathrm{dFdU}$ nucleotides. If this hypothesis can be confirmed in other tissues, a pharmacological basis may be provided for why differences in $C D A$ activity, by modifying the $\mathrm{dFdC} / \mathrm{dFdU}$ ratio, have a substantial impact on the efficacy and toxicity of gemcitabine therapy.

In conclusion, the present study provides the first complete picture of all nucleotides that are formed intracellularly during gemcitabine treatment. Although dFdU was present at higher concentrations than gemcitabine in PBMCs, low intracellular dFdUMP, dFdUDP and dFdUTP concentrations were found. This calls into question the relevance of these dFdU nucleotides for the cytotoxic effects of gemcitabine. 


\section{REFERENCES}

[1] Mackey JR, Mani RS, Selner M, et al. Functional nucleoside transporters are required for gemcitabine influx and manifestation of toxicity in cancer cell lines. Cancer Res 1998;58:4349-57.

[2] Ritzel MW, Ng AM, Yao SY, et al. Recent molecular advances in studies of the concentrative $\mathrm{Na}$-dependent nucleoside transporter (CNT) family: identification and characterization of novel human and mouse proteins ( $\mathrm{hCNT3}$ and $\mathrm{mCNT3}$ ) broadly selective for purine and pyrimidine nucleosides. Mol Membr Biol 2001;18:65-72.

[3] Wong A, Soo RA, Yong W-P, et al. Clinical pharmacology and pharmacogenetics of gemcitabine. Drug Metab Rev 2009;41:7788.

[4] Gandhi V, Legha J, Chen F, et al. Excision of 2',2'-difluorodeoxycytidine (gemcitabine) monophosphate residues from DNA. Cancer Res 1996;56:4453-4459.

[5] Huang P, Chubb S, Hertel L, et al. Action of 2', 2 '-difluorodeoxycytidine on DNA synthesis. Cancer Res 1991;51:6110-6117.

[6] Mini E. Cellular pharmacology of gemcitabine. Ann Oncol 2006;17:7-12.

[7] Heinemann V, Xu YZ, Chubb S, et al. Inhibition of ribonucleotide reduction in CCRF-CEM cells by 2',2'-difluorodeoxycytidine. Mol Pharmacol 1990;38:567-572.

[8] de Sousa Cavalcante L, Monteiro G. Gemcitabine: Metabolism and molecular mechanisms of action, sensitivity and chemoresistance in pancreatic cancer. Eur $J$ Pharmacol 2014;741:8-16.

[9] Heinemann V, Xu YZ, Chubb S, et al. Cellular elimination of 2',2'-difluorodeoxycytidine 5'-triphosphate: A mechanism of selfpotentiation. Cancer Res 1992;52:533-539.

[10] Xu Y-Z, Plunkett W. Modulation of deoxycytidylate deaminase in intact human leukemia cells. Action of 2',2'-difluorodeoxycytidine. Biochem Pharmacol 1992;44:1819-1827.

[11] Veltkamp SA, Pluim D, van Eijndhoven MAJ, et al. New insights into the pharmacology and cytotoxicity of gemcitabine and 2',2'-difluorodeoxyuridine. Mol Cancer Ther 2008;7:2415-25.
[12] Honeywell RJ, Ruiz Van Haperen VWT, Veerman G, et al. Inhibition of thymidylate synthase by 2',2'-difluoro-2'-deoxycytidine (Gemcitabine) and its metabolite 2',2'-difluoro-2'-deoxyuridine. Int J Biochem Cell Biol 2015;60:73-81.

[13] Grunewald R, Kantarjian H, Keating MJ, et al. Pharmacologically directed design of the dose rate and schedule of 2',2'-difluorodeoxycytidine (Gemcitabine) administration in leukemia. Cancer Res 1990;50:6823-6.

[14] Abbruzzese JL, Grunewald R, Weeks EA, et al. A phase I clinical, plasma, and cellular pharmacology study of gemcitabine. J Clin Oncol 1991;9:491-8.

[15] Grunewald R, Abbruzzese JL, Tarassoff P, et al. Saturation of 2',2'-difluorodeoxycytidine 5'-triphosphate accumulation by mononuclear cells during a phase I trial of gemcitabine. Cancer Chemother Pharmacol 1991;27:258262.

[16] Grunewald R, Kantarjian H, Du M, et al. Gemcitabine in leukemia: a phase I clinical, plasma, and cellular pharmacology study. $J$ Clin Oncol 1992;10:406-13.

[17] Patel SR, Gandhi V, Jenkins J, et al. Phase II clinical investigation of gemcitabine in advanced soft tissue sarcomas and window evaluation of dose rate on gemcitabine triphosphate accumulation. J Clin Oncol 2001;19:3483-9.

[18] Gandhi V, Plunkett W, Du M, et al. Prolonged infusion of gemcitabine: Clinical and pharmacodynamic studies during a phase I trial in relapsed acute myelogenous leukemia. J Clin Oncol 2002;20:665-673.

[19] Cattel L, Airoldi M, Delprino L, et al. Pharmacokinetic evaluation of gemcitabine and 2',2'-difluorodeoxycytidine-5'triphosphate after prolonged infusion in patients affected by different solid tumors. Ann Oncol 2006;17 Suppl 5:v142-7.

[20] Grimison P, Galettis P, Manners S, et al. Randomized crossover study evaluating the effect of gemcitabine infusion dose rate: evidence of auto-induction of gemcitabine accumulation. J Clin Oncol 2007;25:5704-9. 
[21] Rizzuto I, Ghazaly E, Peters GJ. Pharmacological factors affecting accumulation of gemcitabine's active metabolite, gemcitabine triphosphate. Pharmacogenomics 2017;18:911-925.

[22] van der Noll R, Smit WM, Wymenga ANM, et al. Phase I and pharmacological trial of lapatinib in combination with gemcitabine in patients with advanced breast cancer. Invest New Drugs 2015;33:1197-205.

[23] Vainchtein LD, Rosing $H$, Thijssen $B$, et al. Validated assay for the simultaneous determination of the anti-cancer agent gemcitabine and its metabolite 2',2'-difluorodeoxyuridine in human plasma by high-performance liquid chromatography with tandem mass spectrometry. Rapid Commun Mass Spectrom 2007;21:2312-22.

[24] Jansen RS, Rosing $H$, Schellens JHM, et al. Simultaneous quantification of 2', 2'-difluorodeoxycytidine and 2',2'-difluorodeoxyuridine nucleosides and nucleotides in white blood cells using porous graphitic carbon chromatography coupled with tandem mass spectrometry. Rapid Commun Mass Spectrom 2009;23:3040-50.

[25] Veltkamp SA, Hillebrand MJX, Rosing $H$, et al. Quantitative analysis of gemcitabine triphosphate in human peripheral blood mononuclear cells using weak anionexchange liquid chromatography coupled with tandem mass spectrometry. J Mass Spectrom 2006;41:1633-42.

[26] Bradford MM. A rapid and sensitive method for the quantitation of microgram quantities of protein utilizing the principle of protein-dye binding. Anal Biochem 1976;72:248-254.

[27] Pluim D, Jacobs BAW, Krähenbühl MD, et al. Correction of peripheral blood mononuclear cell cytosolic protein for hemoglobin contamination. Anal Bioanal Chem 2013;405:2391-5.

[28] Jansen RS, Rosing $H$, Schellens JHM, et al. Retention studies of 2'-2'-difluorodeoxycytidine and 2'-2'-difluorodeoxyuridine nucleosides and nucleotides on porous graphitic carbon: Development of a liquid chromatographytandem mass spectrometry method. J Chromatogr A 2009;1216:3168-3174.
[29] Simiele M, D’Avolio A, Baietto L, et al. Evaluation of the mean corpuscular volume of peripheral blood mononuclear cells of HIV patients by a coulter counter to determine intracellular drug concentrations. Antimicrob Agents Chemother 2011;55:2976-2978.

[30] Cerbone L, Sternberg CN, Sengeløv L, et al. Results from a Phase I Study of Lapatinib with Gemcitabine and Cisplatin in Advanced or Metastatic Bladder Cancer: EORTC Trial 30061. Oncology 2016;90:21-28.

[31] Schultheis B, Kummer G, Zeth M, et al. Phase IB study of sorafenib in combination with gemcitabine and cisplatin in patients with refractory solid tumors. Cancer Chemother Pharmacol 2012;69:333-339.

[32] Carmichael J, Allerheiligen S, Walling J. A phase I study of gemcitabine and carboplatin in non-small cell lung cancer. Semin Oncol 1996;23:55-9.

[33] Kroep JR, Giaccone G, Voorn DA, et al. Gemcitabine and paclitaxel: pharmacokinetic and pharmacodynamic interactions in patients with non-small-cell lung cancer. J Clin Oncol 1999;17:2190-7.

[34] Soo RA, Wang LZ, Tham LS, et al. A multicentre randomised phase II study of carboplatin in combination with gemcitabine at standard rate or fixed dose rate infusion in patients with advanced stage non-small-cell lung cancer. Ann Oncol 2006;17:1128-1133.

[35] van Moorsel CJ, Kroep JR, Pinedo HM, et al. Pharmacokinetic schedule finding study of the combination of gemcitabine and cisplatin in patients with solid tumors. Ann Oncol 1999;10:441-448.

[36] Tempero M, Plunkett W, Ruiz van Haperen VW, et al. Randomized Phase II Comparison of Dose-Intense Gemcitabine: Thirty-Minute Infusion and Fixed Dose Rate Infusion in Patients With Pancreatic Adenocarcinoma. J Clin Oncol 2003;21:3402-3408.

[37] Ruiz van Haperen VW, Veerman G, Eriksson S, et al. Development and molecular characterization of a 2',2'-difluorodeoxycytidine-resistant variant of the human ovarian carcinoma cell line A2780. Cancer Res 1994;54:4138-43.

[38] Hodge LS, Taub ME, Tracy TS. The deaminated metabolite of gemcitabine, 2',2'-difluorodeoxyuridine, modulates the rate of gemcitabine transport and intracellular phosphorylation via deoxycytidine kinase. Drug Metab Dispos 2011;39:2013-6. 
[39] Wang L, Munch-Petersen B, Herrström Sjöberg $A$, et al. Human thymidine kinase 2: Molecular cloning and characterisation of the enzyme activity with antiviral and cytostatic nucleoside substrates. FEBS Lett 1999;443:170-174.

[40] Eriksson S, Arnér E, Spasokoukotskaja T, et al. Properties and levels of deoxynucleoside kinases in normal and tumor cells; implications for chemotherapy. Adv Enzyme Regul 1994;34:13-25.

[41] Gandhi V, Xu YZ, Estey E. Accumulation of arabinosyluracil 5'-triphosphate during arabinosylcytosine therapy in circulating blasts of patients with acute myelogenous leukemia. Clin Cancer Res 1998;4:1719-26.

[42] Ciccolini J, Dahan L, André N, et al. Cytidine deaminase residual activity in serum is a predictive marker of early severe toxicities in adults after gemcitabine-based chemotherapies. J Clin Oncol 2010;28:160165 .
[43] Tibaldi C, Giovannetti E, Vasile E, et al. Correlation of CDA, ERCC1, and XPD polymorphisms with response and survival in gemcitabine/cisplatin-treated advanced nonsmall cell lung cancer patients. Clin Cancer Res 2008;14:1797-1803.

[44] Tibaldi C, Giovannetti E, Tiseo M, et al. Correlation of cytidine deaminase polymorphisms and activity with clinical outcome in gemcitabine-/platinum-treated advanced non-small-cell lung cancer patients. Ann Oncol 2012;23:670-677.

[45] Serdjebi C, Seitz J-F, Ciccolini J, et al. Rapid deaminator status is associated with poor clinical outcome in pancreatic cancer patients treated with a gemcitabine-based regimen. Pharmacogenomics 2013;14:1047-51. 


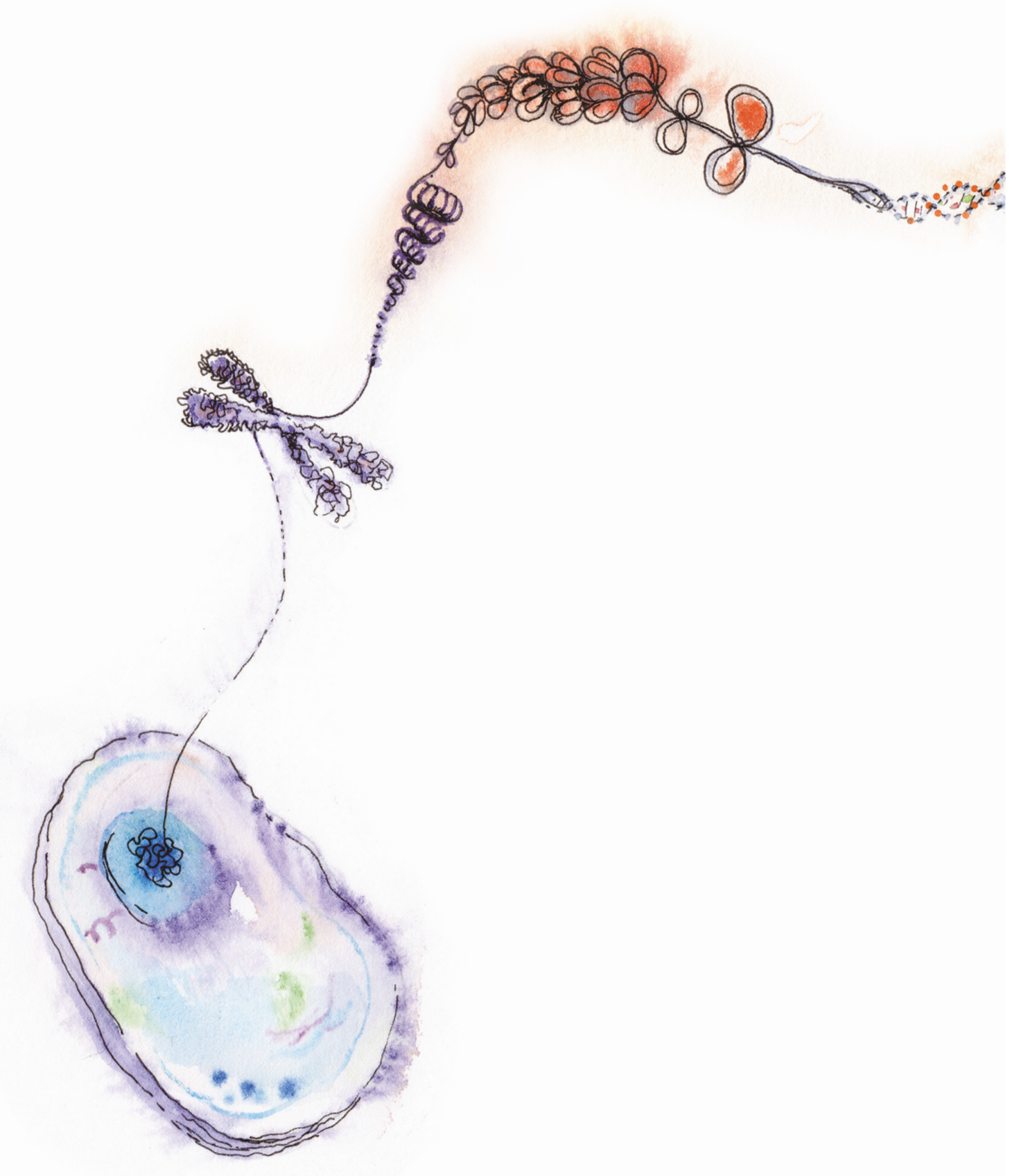




\section{Summary, Conclusions and Perspectives}

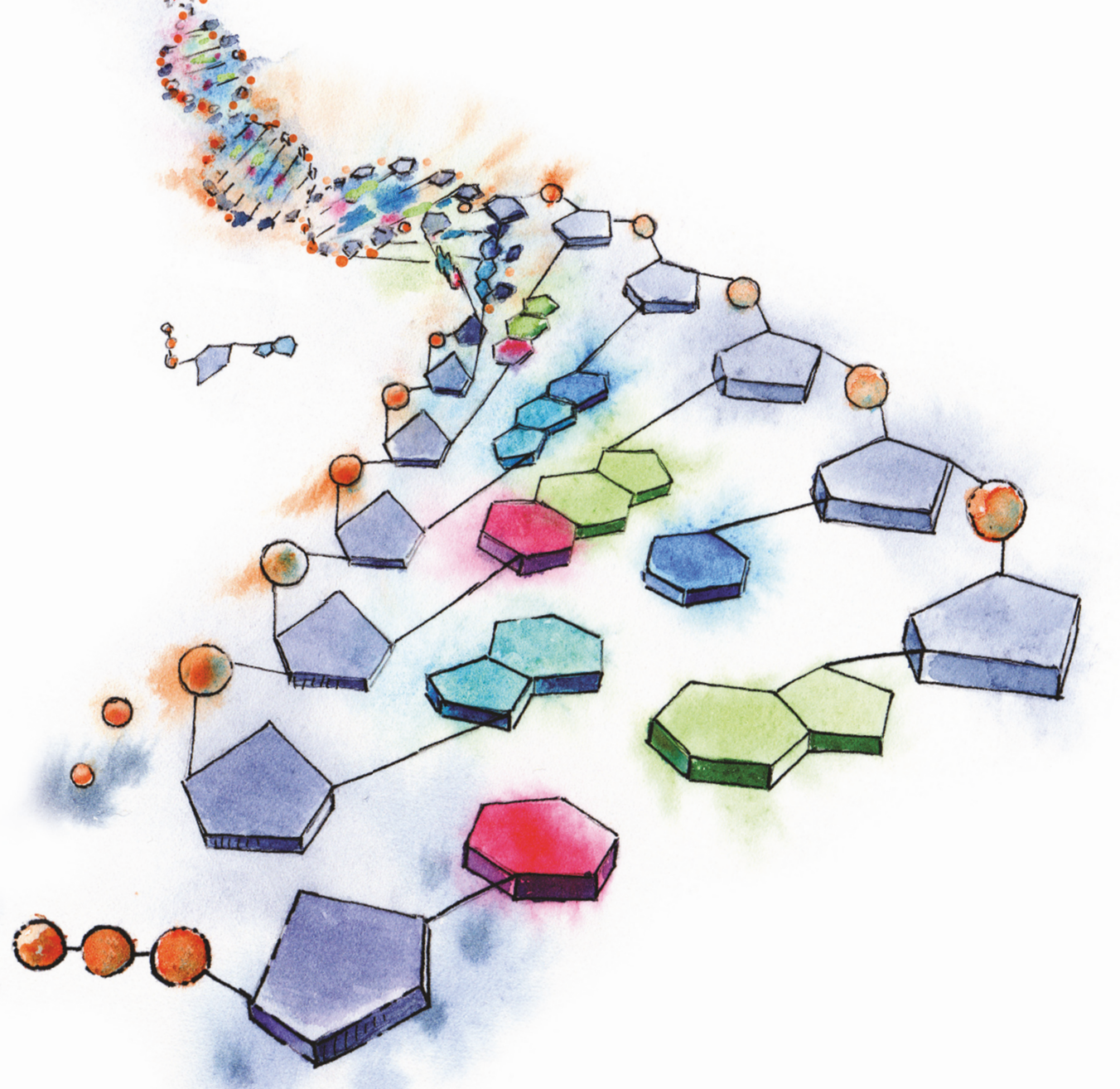




\section{SUMMARY, CONCLUSIONS AND PERSPECTIVES}

\section{What was done prior to this thesis and what was missing...}

Chapter 1 of this thesis provides an overview of the previously conducted clinical trials in which intracellular nucleotide concentrations of pyrimidine analogues were studied in cancer patients. The aim of this review was to gain insight into the parallels in the intracellular pharmacokinetics for the various pyrimidine analogues. This final chapter of the thesis starts with the most important findings of this review to provide a structured overview of what had already been done prior to this thesis and what was still missing. Then, the contribution of the research described in this thesis will be explained. Finally some suggestions for further research will be made.

For cytarabine and gemcitabine, several studies investigated the intracellular pharmacokinetics in patients [1-18]. These studies examined the intracellular cytarabine triphosphate concentrations in leukemic cells and the intracellular gemcitabine triphosphate concentrations in peripheral blood mononuclear cells (PBMCs). PBMCs were used as a surrogate matrix to reflect the intracellular metabolic activation because, unlike solid tumour cells, these cells could be easily obtained at multiple time points after drug administration. For both cytarabine and gemcitabine, there was a clear relationship between the dose and the plasma concentration. However, no clear relationship was found between the plasma concentration and the intracellular triphosphate concentration $[1,2,11,13,19]$. After an identical cytarabine or gemcitabine dose, the intracellularly measured triphosphate concentrations differed considerably between patients $[2,11,19]$.

The intracellular accumulation of the triphosphates also depends on the dosage regimen. For both gemcitabine and cytarabine, it has been shown that saturation of intracellular triphosphate formation occurs from a certain plasma concentration $[2,11,13,19]$.

For cytarabine it was already recognized in 1987 that at plasma concentrations just above $7 \mu \mathrm{M}$ saturation of the intracellular cytarabine triphosphate formation in leukemic cells occurred $[2,11]$. Steady state plasma concentrations which 
were achieved with infusion rates of $200-250 \mathrm{mg} / \mathrm{m}^{2} / \mathrm{h}$ [2,11]. Initially, however, not much was done with this finding. Until 2011, high-dose cytarabine regimens remained common practice for the treatment of patients with acute myeloid leukaemia (AML) [20-22]. High cytarabine doses were given with a high infusion rate, typically $3000 \mathrm{mg} / \mathrm{m}^{2}$ over $1-3$ hours. These dosing regimens led to peak plasma concentrations that were about 5 to 15 times higher than the plasma concentrations that caused saturation of intracellular triphosphate formation [1,23-25]. From a pharmacological perspective, not rational. It was only in 2011 that clinical research by Löwenberg et al. showed that an intermediate-dose remission induction regimen with an infusion rate just above the 'saturation infusion rate' was as effective as high-dose cytarabine, but was associated with less toxicity [26]. In retrospect, you may wonder why it took so long before the findings from clinical pharmacological research were translated into clinical practice.

Following the experience with cytarabine, after the introduction of gemcitabine in 1995, research quickly focused on the steady state plasma concentrations that led to saturation of the intracellular triphosphate formation. Steady state plasma concentrations of $20 \mu \mathrm{M}$ were found to saturate the intracellular formation of gemcitabine triphosphate in PBMCs $[13,14,19]$. Concentrations that were achieved with an infusion rate of $10 \mathrm{mg} / \mathrm{m}^{2} / \mathrm{min}[13,14,19]$. In addition, several studies showed that the intracellular accumulation of gemcitabine triphosphate in PBMCs increased by giving more prolonged gemcitabine infusions at this infusion rate which saturates the formation of gemcitabine triphosphate $[3,18,27]$. However, these prolonged so-called 'fixed dose rate' regimes proved no more effective than conventional 30-minute gemcitabine infusions $[9,28]$. The maximum tolerated dose (MTD) for prolonged infusions of gemcitabine at a fixed dose rate of $10 \mathrm{mg} / \mathrm{m}^{2} / \mathrm{min}$ is lower than the MTD for conventional 30-minute infusions, due to myelotoxicity [29-31]. Efficient administration of gemcitabine, aimed at maximum triphosphate formation, thus also means that less gemcitabine can be administered before dose limiting toxicities are achieved. This means that prolonged administration de facto has no added value.

The relationship between intracellularly formed triphosphates and the cytotoxic effects of pyrimidine analogues is plausible based on their pharmacology. 
However, only few clinical studies have actually investigated the relationship between the intracellular pyrimidine analogue triphosphate concentrations in cells of patients and the clinical therapeutic effect or the occurrence of side effects $[4,6,9,12,32-34]$. For cytarabine, it has been demonstrated that there is a relationship between the intracellular cytarabine triphosphate concentration in leukemic blasts and the response rate in patients with AML [33]. For gemcitabine, the relationship between intracellular triphosphate concentrations and the therapeutic effect has only been demonstrated in tumour cell lines and xenograft studies [35], thus not in patients.

The above shows that for cytarabine and gemcitabine a lot of research has been done on the intracellular pharmacokinetics of the formed triphosphates. However, until the advent of this thesis, little or no research had been done on the intracellular pharmacokinetics of 5-fluorouracil (5-FU), azacitidine and decitabine.

For 5-FU, our knowledge about the intracellular metabolism was mainly based on in vitro studies and animal studies [36-39]. The intracellular pharmacokinetics studies that were conducted in patients in the 1980s and 1990s, were limited to the measurement of 5-fluoro-2'-deoxyuridine monophosphate (FdUMP): only one of the three active nucleotides [40-42]. In these studies a competitivebinding assay was used with bacterial thymidylate synthase as binding protein. The displacement of radiolabelled $\left[6-{ }^{3} \mathrm{H}\right]$-FdUMP was measured by scintillation counting [40-43]. The other two intracellularly formed nucleotides that are responsible for the effect of 5-FU, 5-fluorouridine triphosphate (FUTP) and 5-fluoro-2'-deoxyuridine triphosphate (FdUTP), had never been quantified in patients due to the lack of a sufficiently sensitive bio-analytical assay.

For azacitidine, no research had been conducted to study the intracellular pharmacokinetics of the active nucleotides prior to this thesis. This was also directly related to the lack of a suitable bio-analytical assay.

For decitabine, only two small, exploratory studies were conducted that measured the intracellular concentrations of the decitabine nucleotides in bone marrow cells and PBMCs in a small number of patients $[44,45]$. In both studies, a trend was noticed that higher intracellular decitabine triphosphate 
concentrations were measured in patients who responded well to decitabine treatment than in patients who did not respond $[44,45]$. However, the studies were too small to draw conclusions.

\section{The contribution of this thesis - Part 1. Bio-analytical assay development}

Prior to this thesis, there were no suitable bio-analytical assays for quantitative determination of the pharmacologically active nucleotides of 5-FU and azacitidine in cells of patients. Insight into the intracellular pharmacokinetics is of course also useful for these pyrimidine analogues. That is why we developed these missing assays.

Chapter 2.2 describes the development of a bio-analytical assay for quantitative determination of azacitidine triphosphate in PBMCs. To quantify azacitidine triphosphate in cells, separation from the physiological nucleotides is required. This proved to be a bigger challenge than it had been for the nucleotides of other pyrimidine analogues, because azacitidine triphosphate is very similar to the naturally occurring nucleotides uridine triphosphate and cytidine triphosphate. The monoisotopic molecular masses of azacitidine triphosphate, uridine triphosphate and the naturally occurring $\left[{ }^{13} \mathrm{C}\right]$ - and $\left[{ }^{15} \mathrm{~N}\right]$-isotopes of cytidine triphosphate differ less than $0.02 \mathrm{Da}$. Efforts to select a specific $\mathrm{MS}^{2}$-fragment for azacitidine triphosphate using a triple quadrupole mass spectrometer remained without success. Therefore, we investigated whether separation of azacitidine triphosphate from uridine triphosphate and the $\left[{ }^{13} \mathrm{C}\right]$ - and $\left[{ }^{15} \mathrm{~N}\right]$ isotopes of cytidine triphosphate could be obtained with high-resolution mass spectrometry using a linear trap quadrupole coupled with an Orbitrap. We succeeded in developing the first suitable bio-analytical assay for the quantitative determination of azacitidine triphosphate in PBMCs, by using liquid chromatography coupled with high-resolution mass spectrometry. The assay was successfully applied to measure the intracellular azacitidine triphosphate concentrations in PBMCs of two patients that were treated with azacitidine.

Chapter 3 focuses on the fluoropyrimidines 5-FU and capecitabine. Three intracellularly formed nucleotides are responsible for the antineoplastic effects of these agents: FUTP, FdUTP and FdUMP. Chapter $\mathbf{3 . 1}$ describes the development 
of a liquid chromatography - tandem mass spectrometry (LC-MS/MS) assay for quantitative determination of these three pharmacologically active 5-FU nucleotides in PBMCs. During assay development, it became clear that the 5-FU nucleotide concentrations in cells of patients treated with 5-FU or capecitabine are extremely low. This meant that maximization of the release from the cell matrix and minimization of interference were critical factors to obtain an assay that was sufficiently sensitive. A series of experiments was performed to select the best method for cell lysis and nucleotide extraction. Chromatography was optimized to obtain separation from endogenous nucleotides, and the effect of different cell numbers was examined. The assay was validated and successfully applied to quantify 5-FU nucleotides in PBMCs of patients treated with 5-FU and patients treated with capecitabine. Thirty minutes after an intravenous 5-FU bolus of $400 \mathrm{mg} / \mathrm{m}^{2}$, intracellular FUTP amounts up to $3054 \mathrm{fmol} / 10^{6}$ PBMCs and FdUMP levels up to $169 \mathrm{fmol} / 10^{6}$ PBMCs were measured. The FdUTP concentrations were below the lower limit of quantification. This means that even more sensitive analytical equipment may be needed for measurement of FdUTP in patient samples. The results obtained for patients that were treated with capecitabine are discussed below in the section on Chapter 3.2. To our knowledge, this was the first time that 5-FU nucleotides were quantified in cells of patients treated with 5-FU or capecitabine without using a radiolabel.

\section{The contribution of this thesis - Part 2. Clinical studies exploring the intracellular pharmacokinetics}

In Chapter 3.2 we used the LC-MS/MS assay, developed in Chapter 3.1, to investigate the intracellular pharmacokinetics of the pharmacologically active 5-FU nucleotides in patients treated with capecitabine. To this end, plasma and PBMC samples were collected from 13 patients that received a one-time capecitabine dose of $1000 \mathrm{mg}$ QD and eight patients that were treated with capecitabine $850 \mathrm{mg} / \mathrm{m}^{2}$ BID for 14 days, every three weeks. Samples were collected on day 1 of the treatment and for four patients in the latter group also on day 14 of the treatment. Only FUTP could be measured in the PBMC samples. The FdUTP and FdUMP concentrations were below the detection limits of the assay. Apparently, capecitabine is mainly converted into FUTP and to a lesser extent into FdUTP and FdUMP. This does not necessarily mean that the RNA pathway has a more prominent role in the mechanism of action of capecitabine 
than the DNA pathway. It is known that the natural deoxyribonucleotides are also present in cells in much lower concentrations than the ribonucleotides $[46,47]$. This means that low FdUTP and FdUMP concentrations may still have an important role. However, for the quantification of FdUMP and FdUTP during capecitabine treatment, an even more sensitive bio-analytical assay may be required.

For FUTP, this was the first time that its intracellular concentrations were measured during capecitabine treatment. Consistent with the previously studied pyrimidine analogues, no significant correlation was found between the 5-FU plasma exposure and the intracellular FUTP exposure. The intracellular FUTP concentration-time profiles demonstrated considerable inter-individual variation. An important finding of this study was that FUTP remained considerably longer present in the cells than capecitabine and 5-FU in plasma. After $24 \mathrm{~h}$, intracellular FUTP concentrations were still $41 \%$ of the peak concentrations. Consistent with this finding, after 14 days of treatment with capecitabine twice daily, accumulation of FUTP was found in PBMCs. The area under the FUTP concentration-time curve was found to be significantly increased on day 14 of the treatment compared to day 1. Interestingly, the intracellular FUTP concentrations measured after 14 days of treatment with capecitabine were in the same order of magnitude as the concentrations measured 30 minutes after an intravenous $5-\mathrm{FU}$ bolus of $400 \mathrm{mg} / \mathrm{m}^{2}$.

This was the first time that the intracellular pharmacokinetics of the three pharmacologically active 5-FU nucleotides were explored during capecitabine treatment. Of course, we have to keep in mind that the nucleotide concentrations measured in PBMCs may be different than the concentrations that would be found in tumour cells. Especially since capecitabine has a certain degree of tumour selectivity. The enzyme responsible for the third step in the conversion of capecitabine to 5-FU is more present in tumour tissue [48].

Chapter 4.1 focuses on the intracellular pharmacokinetics of gemcitabine. As described at the beginning of this chapter, gemcitabine is one of the pyrimidine analogues for which the intracellular pharmacokinetics has been studied more often. Several studies determined the intracellular concentrations of gemcitabine triphosphate during gemcitabine treatment $[3,4,6,7,9,10,13,14,16-18,27]$. 
In plasma, gemcitabine is rapidly converted to 2',2'-difluoro-2'-deoxyuridine (dFdU) by the enzyme cytidine deaminase [13]. Question is whether dFdU also contributes to the pharmacological effects of gemcitabine. To clarify the role of $\mathrm{dFdU}$, we investigated the intracellular pharmacokinetics of both the nucleotides of gemcitabine (i.e. gemcitabine monophosphate, diphosphate and triphosphate) and the nucleotides of $d F d U$ (i.e. dFdU monophosphate, diphosphate and triphosphate) in patients treated with gemcitabine. Intracellular concentrations were determined in PBMCs obtained from 38 patients treated with gemcitabine $500-1000 \mathrm{mg} / \mathrm{m}^{2}$, infused in 30 minutes. To this end, we used the LC-MS/MS assay previously described by our group [49]. In all patients, the dFdU plasma exposure was much higher than the gemcitabine plasma exposure. Also in PBMCs, dFdU was present in higher concentrations than gemcitabine. However, dFdU was phosphorylated only to a limited extent. The intracellular dFdU nucleotide concentrations in PBMCs were much lower than the gemcitabine nucleotide concentrations. This suggests that the contribution of dFdU nucleotides to the cytotoxic effect of gemcitabine is limited. Further research should tell whether $\mathrm{dFdU}$ is also hardly phosphorylated in tumour cells. If dFdU hardly contributes to the cytotoxic effects of gemcitabine, due to limited intracellular activation, inter-individual differences in cytidine deaminase activity might lead to substantial differences in the efficacy and toxicity of gemcitabine therapy between patients. This was indeed seen in a number of clinical studies [50-53]. 


\section{Conclusions}

Using the latest analytical techniques, two sophisticated bio-analytical assays were developed for quantitative determination of the pharmacologically active nucleotides of azacitidine and 5-FU, respectively, in cells of patients.

After treatment with capecitabine, only FUTP could be measured in PBMCs. Apparently, capecitabine is mainly converted into FUTP and to a lesser extent into FdUTP and FdUMP. There was no clear relationship between the 5-FU plasma exposure and the intracellular FUTP exposure. FUTP remained much longer present in PBMCs than 5-FU in plasma. During 14 days of treatment with capecitabine twice daily intracellular accumulation of FUTP occurs.

After treatment with gemcitabine, substantial intracellular dFdU concentrations were measured in PBMCs. However, dFdU was only phosphorylated to a very limited extent in PBMCs. Intracellular dFdU nucleotide concentrations were much lower than the gemcitabine nucleotide concentrations. This suggests that the contribution of dFdU nucleotides to the cytotoxic effect of gemcitabine is limited.

When patients were treated with an identical capecitabine or gemcitabine dose, considerable inter-individual differences were seen in the intracellular nucleotide exposure in PBMCs. In part these inter-individual differences can be traced back to differences in plasma exposure. However, the inter-individual variations for the intracellular FUTP, gemcitabine diphosphate and gemcitabine triphosphate exposure were higher than the variations that were seen in plasma. 


\section{Future perspectives}

Despite the advent of modern anticancer agents, the pyrimidine analogues are still a mainstay in the treatment of many oncological diseases. They have often been part of a treatment approach for decades. Over the years, treatment regimens have evolved into the regimens that are currently applied. Nothing more sophisticated than the maximum tolerated dose has usually been the basis for these treatment regimens. Research that has studied the pharmacology of the pyrimidine analogues has often been conducted decades ago. Meanwhile, we have many new analytical techniques at our disposal. In my opinion, it is useful to use these new analytical techniques to further study the current therapy with pyrimidine analogues and thereby update our knowledge about their clinical pharmacology.

Clinical research to achieve better treatment regimens should, in my opinion, go much more hand in hand with pharmacological research. It is important to think mechanistically about what we see in our clinical practice and what we can do to optimize therapy. Situations where there is a gap between the knowledge of the pharmacology and clinical practice, as with the high-dose cytarabine regimens that were common practice until 2011, must be avoided. The exact opposite is what we strive for. New insights into pharmacology should be a rationale to improve the clinically applied regimens. This requires collaboration between the various disciplines.

Therapy individualization is an important theme in oncology and brings us more and more targeted therapies. Question is why we make no greater effort to individualize also the therapy with classical cytostatics. Chapters 3.2 and 4.1 of this thesis have shown that when patients were treated with an identical capecitabine or gemcitabine dose, considerable inter-individual differences were seen in both plasma levels and the intracellular nucleotide levels in PBMCs. Such large differences in drug levels between patients (both in plasma and in cells) should not be taken for granted; especially since the effect of the treatment can only be evaluated after a number of treatment cycles. We should investigate which drug levels are desirable in order to pursue these levels. After all, the large inter-individual differences in plasma and intracellular drug concentrations will imply that some of the patients are not treated optimally. If no plasma-effect relationship can be demonstrated, we should not leave it at that, but set up 
clinical pharmacology studies to find out why. It is not rational to assume that the plasma level is totally irrelevant. It is much more likely that many more factors contribute to differences in clinical outcome.

Chapter 4.1 of this thesis demonstrated that dFdU is only phosphorylated to a very limited extent in PBMCs. Future studies should show whether dFdU is also hardly phosphorylated in tumour cells. If this is the case, dFdU is unlikely to contribute substantially to the cytotoxic effects of gemcitabine. In this light, it would be rational to pay more attention to inter-individual differences in the deamination rate of gemcitabine to $\mathrm{dFdU}$. Some clinical studies have shown that gemcitabine therapy was more effective and more toxic in patients with low cytidine deaminase activity, i.e. a decreased deamination of gemcitabine to dFdU [50-52]. Future studies should show whether individualization of the gemcitabine dose, based on the measured cytidine deaminase activity, leads to better treatment results.

Based on the pharmacology of the pyrimidine analogues, a relationship is expected between the intracellular concentrations of the active nucleotides and the cytotoxic effects. However, for most pyrimidine analogues, this relationship has only been demonstrated in in vitro studies and animal studies [35]. Only for cytarabine, this relationship has been confirmed in patients [33]. It is important, also for the other pyrimidine analogues, to prove in future clinical studies that there is indeed a relationship between the intracellular nucleotide concentrations and the clinical outcome. It is useful to examine both the relationship between the intracellular nucleotide concentrations and efficacy and the relationship between the intracellular nucleotide concentrations and toxicity. In this way, an 'intracellular therapeutic window' may be defined.

If the relationship between the intracellular nucleotide concentrations and the therapeutic effect can be confirmed in patients, this offers several new opportunities. One of the opportunities is to use the intracellular nucleotide concentration in PBMCs as a predictive marker for the therapeutic effect. If the intracellular nucleotide concentrations are low after the first dose, this indicates that not much effect of the therapy is to be expected. A decision can then be made whether the pyrimidine analogue is given in a higher dose or is switched to a different treatment. Patients with inadequate intracellular nucleotide 
concentrations then no longer have to undergo a number of fruitless treatment cycles before the treatment effect can be evaluated. This means that these patients can start months earlier with a therapy that, for them, is potentially more effective. Clinical studies will have to proof whether intracellular nucleotide concentrations can indeed be used as a predictive marker.

If an 'intracellular therapeutic window' can be defined, the possibility of therapeutic drug monitoring based on the measured intracellular nucleotide concentrations could also be investigated. Based on the measured intracellular nucleotide concentrations and the established 'intracellular therapeutic window', a dose adjustment strategy could be developed.

Considering all these possibilities, I can only state that, in addition to investing in new oncolytic drugs, we should also take a careful look at our current therapies. By using state-of-the-art analytical techniques, we can upgrade our knowledge of clinical pharmacology. This knowledge can be used to improve the pharmacotherapy. By not doing this, we will miss an opportunity...

I am confident that this thesis has contributed to a better understanding of the intracellular pharmacokinetics of the pyrimidine analogues used in the treatment of cancer. I also hope that this thesis provides an incentive to use the latest bio-analytical techniques to gain even more insight into the (intracellular) pharmacology in patients. In this way, we can improve therapy based on stateof-the art knowledge of the pharmacology, also for the widely used 'classical' cytostatics. 


\section{REFERENCES}

[1] Liliemark JO, Plunkett W, Dixon DO. Relationship of 1-beta-Darabinofuranosylcytosine in plasma to 1-beta-D-arabinofuranosylcytosine 5'-triphosphate levels in leukemic cells during treatment with high-dose 1-betaD-arabinofuranosylcytosine. Cancer Res 1985;45:5952-7.

[2] Plunkett W, Liliemark JO, Adams $T M$, et al. Saturation of 1-beta-Darabinofuranosylcytosine 5'-triphosphate accumulation in leukemia cells during highdose 1-beta-D-arabinofuranosylcytosine therapy. Cancer Res 1987;47:3005-11.

[3] Gandhi V, Plunkett W, Du M, et al. Prolonged infusion of gemcitabine: Clinical and pharmacodynamic studies during a phase I trial in relapsed acute myelogenous leukemia. J Clin Oncol 2002;20:665-673.

[4] Tempero M, Plunkett W, Ruiz van Haperen VW, et al. Randomized Phase II Comparison of Dose-Intense Gemcitabine: Thirty-Minute Infusion and Fixed Dose Rate Infusion in Patients With Pancreatic Adenocarcinoma. J Clin Oncol 2003;21:3402-3408.

[5] Cattel L, Airoldi M, Delprino L, et al. Pharmacokinetic evaluation of gemcitabine and 2',2'-difluorodeoxycytidine-5'triphosphate after prolonged infusion in patients affected by different solid tumors. Ann Oncol 2006;17 Suppl 5:v142-7.

[6] Soo RA, Wang LZ, Tham LS, et al. A multicentre randomised phase II study of carboplatin in combination with gemcitabine at standard rate or fixed dose rate infusion in patients with advanced stage non-small-cell lung cancer. Ann Oncol 2006;17:1128-1133.

[7] Nieto Y, Aldaz A, Rifón J, et al. Phase I and Pharmacokinetic Study of Gemcitabine Administered at Fixed-Dose Rate, Combined with Docetaxel/Melphalan/Carboplatin, with Autologous Hematopoietic ProgenitorCell Support, in Patients with Advanced Refractory Tumors. Biol Blood Marrow Transplant 2007;13:1324-1337.

[8] Veltkamp SA, Jansen RS, Callies S, et al. Oral administration of gemcitabine in patients with refractory tumors: a clinical and pharmacologic study. Clin Cancer Res 2008;14:3477-86.
[9] Poplin E, Feng Y, Berlin J, et al. Phase III, Randomized Study of Gemcitabine and Oxaliplatin Versus Gemcitabine (fixed-dose rate infusion) Compared With Gemcitabine (30-minute infusion) in Patients With Pancreatic Carcinoma E6201: A Trial of the Eastern Cooperative Oncology Group. J Clin Oncol 2009;27:3778-3785.

[10] Caffo O, Fallani S, Marangon E, et al. Pharmacokinetic study of gemcitabine, given as prolonged infusion at fixed dose rate, in combination with cisplatin in patients with advanced non-small-cell lung cancer. Cancer Chemother Pharmacol 2010;65:1197-202.

[11] Plunkett W, Liliemark JO, Estey E, et al. Saturation of ara-CTP accumulation during high-dose ara-C therapy: pharmacologic rationale for intermediate-dose ara-C. Semin Oncol 1987;14:159-66.

[12] Estey EH, Keating MJ, McCredie KB, et al. Cellular ara-CTP pharmacokinetics, response, and karyotype in newly diagnosed acute myelogenous leukemia. Leukemia 1990;4:959.

[13] Abbruzzese JL, Grunewald R, Weeks EA, et al. A phase I clinical, plasma, and cellular pharmacology study of gemcitabine. J Clin Oncol 1991;9:491-8.

[14] Grunewald R, Kantarjian H, Du M, et al. Gemcitabine in leukemia: a phase I clinical, plasma, and cellular pharmacology study. $J$ Clin Oncol 1992;10:406-13.

[15] Gandhi V, Xu YZ, Estey E. Accumulation of arabinosyluracil 5'-triphosphate during arabinosylcytosine therapy in circulating blasts of patients with acute myelogenous leukemia. Clin Cancer Res 1998;4:1719-26.

[16] Kroep JR, Giaccone G, Voorn DA, et al. Gemcitabine and paclitaxel: pharmacokinetic and pharmacodynamic interactions in patients with non-small-cell lung cancer. J Clin Oncol 1999;17:2190-7.

[17] van Moorsel CJ, Kroep JR, Pinedo HM, et al. Pharmacokinetic schedule finding study of the combination of gemcitabine and cisplatin in patients with solid tumors. Ann Oncol 1999;10:441-448. 
[18] Patel SR, Gandhi V, Jenkins J, et al. Phase II clinical investigation of gemcitabine in advanced soft tissue sarcomas and window evaluation of dose rate on gemcitabine triphosphate accumulation. I Clin Oncol 2001;19:3483-9.

[19] Grunewald R, Abbruzzese JL, Tarassoff P, et al. Saturation of 2',2'-difluorodeoxycytidine 5'-triphosphate accumulation by mononuclear cells during a phase I trial of gemcitabine. Cancer Chemother Pharmacol 1991;27:258262.

[20] Farag SS, Ruppert AS, Mrózek K, et al. Outcome of induction and postremission therapy in younger adults with acute myeloid leukemia with normal karyotype: a cancer and leukemia group B study. J Clin Oncol 2005;23:482-93.

[21] Büchner T, Berdel WE, Schoch C, et al. Double induction containing either two courses or one course of high-dose cytarabine plus mitoxantrone and postremission therapy by either autologous stem-cell transplantation or by prolonged maintenance for acute myeloid leukemia. J Clin Oncol 2006;24:2480-2489.

[22] Döhner H, Estey EHE, Amadori S, et al. Diagnosis and management of acute myeloid leukemia in adults: recommendations from an international expert panel, on behalf of the European LeukemiaNet. Blood 2010;115:453474.

[23] Breithaupt $H$, Pralle $H$, Eckhardt $T$, et al. Clinical results and pharmacokinetics of high-dose cytosine arabinoside (HD ARA-C). Cancer 1982;50:1248-57.

[24] Early AP, Preisler HD, Slocum H, et al. A pilot study of high-dose 1-beta-Darabinofuranosylcytosine for acute leukemia and refractory lymphoma: clinical response and pharmacology. Cancer Res 1982;42:1587-94.

[25] Capizzi RL, Yong JL, Cheng E. Alteration of the pharmacokinetics of high-dose Ara-C by its metabolite, high Ara- $U$ in patients with acute leukemia. J Clin Oncol 1983;1:763-771.

[26] Löwenberg B, Pabst T, Vellenga E, et al. Cytarabine dose for acute myeloid leukemia. N Engl J Med 2011;364:1027-1036.

[27] Cattel L, Airoldi M, Delprino L, et al. Pharmacokinetic evaluation of gemcitabine and 2',2'-difluorodeoxycytidine-5'triphosphate after prolonged infusion in patients affected by different solid tumors. Ann Oncol 2006;17 Suppl 5:v142-7.
[28] Gridelli C, Gallo C, Ceribelli A, et al. Factorial phase III randomised trial of rofecoxib and prolonged constant infusion of gemcitabine in advanced non-small-cell lung cancer: the GEmcitabine-COxib in NSCLC (GECO) study. Lancet Oncol 2007;8:500-512.

[29] Fossella F V, Lippman SM, Shin DM, et al. Maximum-tolerated dose defined for singleagent gemcitabine: a phase I dose-escalation study in chemotherapy-naive patients with advanced non-small-cell lung cancer. J Clin Oncol 1997;15:310-6.

[30] Brand R, Capadano M, Tempero M. A phase I trial of weekly gemcitabine administered as a prolonged infusion in patients with pancreatic cancer and other solid tumors. Invest New Drugs 1997;15:331-341.

[31] Touroutoglou N, Gravel D, Raber MN, et al. Clinical results of a pharmacodynamicallybased strategy for higher dosing of gemcitabine in patients with solid tumors. Ann Oncol 1998;9:1003-1008.

[32] Kantarjian HM, Estey EH, Plunkett W, et al. Phase I-II clinical and pharmacologic studies of high-dose cytosine arabinoside in refractory leukemia. Am J Med 1986;81:387394.

[33] Estey E, Plunkett W, Dixon D, et al. Variables predicting response to high dose cytosine arabinoside therapy in patients with refractory acute leukemia. Leukemia 1987;1:580-3.

[34] Grimison P, Galettis P, Manners S, et al. Randomized crossover study evaluating the effect of gemcitabine infusion dose rate: evidence of auto-induction of gemcitabine accumulation. J Clin Oncol 2007;25:5704-9.

[35] Ruiz van Haperen VW, Veerman G, Boven $E$, et al. Schedule dependence of sensitivity to 2',2'-difluorodeoxycytidine (gemcitabine) in relation to accumulation and retention of its triphosphate in solid tumour cell lines and solid tumours. Biochem Pharmacol 1994;48:1327-1339.

[36] Peters GJ, Laurensse E, Leyva A, et al. Sensitivity of human, murine, and rat cells to 5-fluorouracil and 5'-deoxy-5-fluorouridine in relation to drug-metabolizing enzymes. Cancer Res 1986;46:20-8.

[37] Ciccolini J, Peillard L, Evrard a, et al. Enhanced antitumor activity of 5-fluorouracil in combination with 2'-deoxyinosine in human colorectal cell lines and human colon tumor xenografts. Clin Cancer Res 2000;6:15291535. 
[38] Ciccolini J, Peillard L, Aubert C, et al. Monitoring of the intracellular activation of 5 -fluorouracil to deoxyribonucleotides in HT29 human colon cell line: application to modulation of metabolism and cytotoxicity study. Fundam Clin Pharmacol 2000;14:147154.

[39] Kamm YJL, Peters GJ, Hull WE, et al. Correlation between 5-fluorouracil metabolism and treatment response in two variants of $\mathrm{C} 26$ murine colon carcinoma. $\mathrm{Br} \mathrm{J}$ Cancer 2003;89:754-62.

[40] Spears CP, Gustavsson BG, Mitchell MS, et al. Thymidylate synthetase inhibition in malignant tumors and normal liver of patients given intravenous 5-fluorouracil. Cancer Res 1984;44:4144-50.

[41] Spears CP, Gustavsson BG, Berne M, et al. Mechanisms of innate resistance to thymidylate synthase inhibition after 5-fluorouracil. Cancer Res 1988;48:58945900.

[42] Peters GJ, Lankelma J, Kok RM, et al. Prolonged retention of high concentrations of 5-fluorouracil in human and murine tumors as compared with plasma. Cancer Chemother Pharmacol 1993;31:269-76.

[43] Moran RG, Spears CP, Heidelberger C. Biochemical determinants of tumor sensitivity to 5-fluorouracil: ultrasensitive methods for the determination of 5-fluoro2'-deoxyuridylate, 2'-deoxyuridylate, and thymidylate synthetase. Proc Natl Acad Sci USA 1979;76:1456-60.

[44] Jansen RS, Rosing H, Wijermans PW, et al. Decitabine triphosphate levels in peripheral blood mononuclear cells from patients receiving prolonged low-dose decitabine administration: A pilot study. Cancer Chemother Pharmacol 2012;69:1457-1466.

[45] Wang $\mathrm{H}$, Chen $\mathrm{P}$, Wang J, et al. In vivo quantification of active decitabinetriphosphate metabolite: a novel pharmacoanalytical endpoint for optimization of hypomethylating therapy in acute myeloid leukemia. AAPS J 2013;15:242-9.
[46] Chen P, Liu Z, Liu S, et al. A LC-MS/MS method for the analysis of intracellular nucleoside triphosphate levels. Pharm Res 2009;26:1504-15.

[47] Cohen S, Megherbi M, Jordheim LP, et al. Simultaneous analysis of eight nucleoside triphosphates in cell lines by liquid chromatography coupled with tandem mass spectrometry. J Chromatogr B Analyt Technol Biomed Life Sci 2009;877:3831-40.

[48] Miwa M, Ura M, Nishida M, et al. Design of a novel oral fluoropyrimidine carbamate, capecitabine, which generates 5 -fluorouracil selectively in tumours by enzymes concentrated in human liver and cancer tissue. Eur J Cancer 1998;34:1274-81.

[49] Jansen RS, Rosing $H$, Schellens JHM, et al. Simultaneous quantification of 2',2'-difluorodeoxycytidine and 2',2'-difluorodeoxyuridine nucleosides and nucleotides in white blood cells using porous graphitic carbon chromatography coupled with tandem mass spectrometry. Rapid Commun Mass Spectrom 2009;23:3040-50.

[50] Ciccolini J, Dahan L, André N, et al. Cytidine deaminase residual activity in serum is a predictive marker of early severe toxicities in adults after gemcitabine-based chemotherapies. J Clin Oncol 2010;28:160165.

[51] Tibaldi C, Giovannetti E, Vasile E, et al. Correlation of CDA, ERCC1, and XPD polymorphisms with response and survival in gemcitabine/cisplatin-treated advanced nonsmall cell lung cancer patients. Clin Cancer Res 2008;14:1797-1803.

[52] Tibaldi C, Giovannetti E, Tiseo M, et al. Correlation of cytidine deaminase polymorphisms and activity with clinical outcome in gemcitabine-/platinum-treated advanced non-small-cell lung cancer patients. Ann Oncol 2012;23:670-677.

[53] Serdjebi C, Seitz J-F, Ciccolini J, et al. Rapid deaminator status is associated with poor clinical outcome in pancreatic cancer patients treated with a gemcitabine-based regimen. Pharmacogenomics 2013;14:1047-51. 


\section{SAMENVATTING, CONCLUSIES EN PERSPECTIEVEN}

\section{Wat was er gedaan voorafgaand aan dit proefschrift en wat ontbrak ...}

Hoofdstuk 1 van dit proefschrift geeft een overzicht van de tot dusver uitgevoerde klinische studies waarin intracellulaire nucleotiden concentraties van pyrimidine analoga werden bestudeerd bij kankerpatiënten. Het doel van deze review was om voor de verschillende pyrimidine analoga inzicht te krijgen in de parallellen in de intracellulaire farmacokinetiek. Dit laatste hoofdstuk begint met de belangrijkste bevindingen van deze review om een gestructureerd overzicht te geven van wat er voorafgaand aan dit proefschrift al was gedaan en wat nog ontbrak. Vervolgens zal de bijdrage van het onderzoek beschreven in dit proefschrift worden toegelicht. Ten slotte zullen enkele suggesties voor verder onderzoek worden gedaan.

Voor cytarabine en gemcitabine is de intracellulaire farmacokinetiek bij patiënten in verschillende studies onderzocht [1-18]. Deze studies bestudeerden de intracellulaire cytarabine-trifosfaat concentraties in leukemiecellen en de intracellulaire gemcitabine-trifosfaat concentraties in mononucleaire cellen uit het perifere bloed (PBMC's). PBMC's werden gebruikt als surrogaatmatrix om de intracellulaire metabole activatie te bestuderen omdat ze, in tegenstelling tot solide-tumorcellen, gemakkelijk op meerdere tijdstippen na toediening van gemcitabine konden worden afgenomen. Voor zowel cytarabine als gemcitabine werd een duidelijke relatie gevonden tussen de dosis en de plasmaconcentratie. Er bleek echter geen duidelijke relatie te bestaan tussen de plasmaconcentratie en de intracellulaire trifosfaat concentratie $[1,2,11,13,19]$. Na een identieke dosis cytarabine of gemcitabine verschilden de intracellulair gemeten trifosfaat concentraties aanzienlijk tussen patiënten $[2,11,19]$.

De intracellulaire accumulatie van trifosfaat is ook afhankelijk van het doseerschema. Voor zowel gemcitabine als cytarabine is aangetoond dat vanaf een bepaalde plasmaconcentratie verzadiging van de intracellulaire trifosfaat vorming optreedt $[2,11,13,19]$. 
Voor cytarabine werd al in 1987 aangetoond dat bij plasmaconcentraties net boven $7 \mu \mathrm{M}$ verzadiging optreedt van de intracellulaire cytarabine-trifosfaat vorming in leukemiecellen; steady-state plasmaconcentraties die werden bereikt met infusiesnelheden van $200-250 \mathrm{mg} / \mathrm{m}^{2} /$ uur [2,11]. In eerste instantie werd er echter niet veel gedaan met deze bevinding. Tot 2011 bleven hoge-dosis cytarabineregimes gebruikelijk voor de behandeling van patiënten met acute myeloïde leukemie (AML) [20-22]. Hierbij werden hoge cytarabine doseringen gegeven met een hoge infusiesnelheid, meestal $3000 \mathrm{mg} / \mathrm{m}^{2}$ toegediend in 1-3 uur. Deze doseerregimes leidden tot piek-plasmaconcentraties die ongeveer 5 tot 15 keer hoger waren dan de plasmaconcentraties die zorgden voor verzadiging van de intracellulaire trifosfaat vorming [1,23-25]. Farmacologisch gezien niet rationeel. Pas in 2011 toonde klinisch onderzoek door Löwenberg et al. aan dat een remissie-inductieregime met een intermediaire dosis en een infusiesnelheid net boven de 'verzadigings-infusiesnelheid' even effectief is als hoog gedoseerd cytarabine, maar gepaard gaat met minder toxiciteit [26]. Achteraf bezien, kun je je afvragen waarom het zo lang heeft geduurd voordat de bevindingen uit het klinisch farmacologisch onderzoek vertaald werden naar de klinische praktijk.

Volgend op de ervaringen met cytarabine, werd er na de introductie van gemcitabine in 1995 al snel onderzoek gedaan naar de steady-state plasmaconcentraties waarbij verzadiging van de intracellulaire gemcitabinetrifosfaat vorming optreedt in PBMC's. Dit bleek het geval bij steady-state plasmaconcentraties boven $20 \mu \mathrm{M}[13,14,19]$. Concentraties die worden bereikt vanaf een infusiesnelheid van $10 \mathrm{mg} / \mathrm{m}^{2} / \mathrm{min}[13,14,19]$. Ook werd in verschillende studies aangetoond dat de intracellulaire accumulatie van gemcitabine-trifosfaat toenam door verlengde infusies met gemcitabine te geven met deze infusiesnelheid welke de vorming van gemcitabine-trifosfaat verzadigt $[3,18,27]$. Toch bleken deze verlengde zogenaamde 'fixed dose rate' regimes niet effectiever dan conventionele, 30 minuten durende, infusies met gemcitabine $[9,28]$. De maximaal getolereerde dosis (MTD) voor verlengde gemcitabine infusies met een vaste inloopsnelheid van $10 \mathrm{mg} / \mathrm{m}^{2} / \mathrm{min}$ is lager dan de MTD voor conventionele infusies van 30 minuten, vanwege myelotoxiciteit [29-31]. Het efficiënt toedienen van gemcitabine, gericht op maximale trifosfaat vorming, betekent dus ook dat er minder gemcitabine kan worden toegediend 
voordat dosis-beperkende toxiciteit wordt bereikt. Dit betekent dat verlengde toediening de facto geen meerwaarde heeft.

De relatie tussen intracellulair gevormde trifosfaten en het cytotoxisch effect van pyrimidine analoga is op grond van de farmacologie aannemelijk. Er zijn echter maar weinig klinische studies die de relatie tussen de intracellulaire pyrimidine analogon trifosfaat concentratie in cellen van patiënten en het klinisch therapeutisch effect, dan wel het optreden van bijwerkingen, daadwerkelijk hebben onderzocht $[4,6,9,12,32-34]$. Voor cytarabine is aangetoond dat er een relatie is tussen de intracellulaire cytarabine-trifosfaat concentraties in leukemische blasten en de kans op respons bij patiënten met AML [33]. Voor gemcitabine is de relatie tussen de intracellulaire trifosfaat concentraties en het therapeutisch effect alleen aangetoond in tumorcellijnen en studies met xenotransplantaten [35], dus niet bij patiënten.

Het bovenstaande laat zien dat voor cytarabine en gemcitabine veel onderzoek is gedaan naar de intracellulaire farmacokinetiek van de gevormde trifosfaten. Tot de komst van dit proefschrift was er echter weinig of geen onderzoek verricht dat de intracellulaire farmacokinetiek van 5-fluorouracil (5-FU), azacitidine en decitabine bestudeerde.

Voor 5-FU was onze kennis over het intracellulaire metabolisme vooral gebaseerd op in vitro studies en dierstudies [36-39]. De intracellulaire farmacokinetiek studies die in de jaren ' 80 en ' 90 zijn uitgevoerd bij patiënten, beperkten zich tot het meten van 5-fluoro-2'-deoxyuridine-monofosfaat (FdUMP): slechts één van de drie actieve nucleotiden [40-42]. In deze studies werd een competitieve bindingsassay gebruikt met bacterieel thymidylaat synthase als bindingseiwit, waarbij de verdringing van radioactief gelabeld $\left[6-{ }^{3} \mathrm{H}\right]-$ FdUMP werd gemeten door scintillatietelling [40-43]. De andere twee intracellulair gevormde nucleotiden die verantwoordelijk zijn voor het effect van 5-FU, 5-fluorouridine-trifosfaat (FUTP) en 5-fluoro-2'-deoxyuridine-trifosfaat (FdUTP), waren tot dusver nooit gekwantificeerd bij patiënten vanwege het ontbreken van een voldoende gevoelige bio-analytische bepalingsmethode.

Voor azacitidine was er, voorafgaand aan dit proefschrift, nog helemaal geen onderzoek verricht naar de intracellulaire farmacokinetiek van de actieve 
nucleotiden. Dit was ook direct gerelateerd aan het ontbreken van een geschikte bio-analytische bepalingsmethode.

Voor decitabine waren er slechts twee kleine, exploratieve studies uitgevoerd die de intracellulaire concentraties van de decitabine nucleotiden maten in beenmergcellen en PBMC's bij een klein aantal patiënten $[44,45]$. In beide studies werd een trend opgemerkt dat bij patiënten die goed respondeerden op de behandeling met decitabine hogere intracellulaire decitabine-trifosfaat concentraties werden gemeten dan bij patiënten die niet respondeerden $[44,45]$. De studies waren echter te klein om eenduidige conclusies te kunnen trekken.

\section{De bijdrage van dit proefschrift - Deel 1. Ontwikkeling van bio- analytische bepalingsmethoden}

Voorafgaand aan dit proefschrift waren er geen geschikte bio-analytische bepalingsmethoden voor het kwantificeren van de farmacologisch actieve nucleotiden van 5-FU en azacitidine in cellen van patiënten. Inzicht in de intracellulaire farmacokinetiek is natuurlijk ook voor deze pyrimidine analoga nuttig. Daarom hebben we deze ontbrekende bepalingsmethoden ontwikkeld.

Hoofdstuk 2.2 beschrijft de ontwikkeling van een bio-analytische bepalingsmethode voor de kwantitatieve bepaling van azacitidine-trifosfaat in PBMC's. Om azacitidine-trifosfaat in cellen te kwantificeren, is scheiding nodig van de fysiologische nucleotiden. Dit bleek een grotere uitdaging dan het is geweest voor de nucleotiden van andere pyrimidine analoga, omdat azacitidinetrifosfaat erg lijkt op de natuurlijk voorkomende nucleotiden uridine-trifosfaat en cytidine-trifosfaat. De monoisotopische molecuulmassa's van azacitidinetrifosfaat, uridine-trifosfaat en de natuurlijk voorkomende $\left[{ }^{13} \mathrm{C}\right]$ - en $\left[{ }^{15} \mathrm{~N}\right]$ isotopen van cytidine-trifosfaat verschillen minder dan 0,02 Da. Pogingen om een specifiek $\mathrm{MS}^{2}$-fragment voor azacitidine-trifosfaat te selecteren met behulp van een triple quadrupool massaspectrometer waren niet succesvol. Daarom hebben we onderzocht of we azacitidine-trifosfaat konden scheiden van uridinetrifosfaat en de $\left[{ }^{13} \mathrm{C}\right]$ - en $\left[{ }^{15} \mathrm{~N}\right]$-isotopen van cytidine-trifosfaat met behulp van hoge-resolutie massaspectrometrie met een lineaire ion-trap gekoppeld aan een Orbitrap. Door gebruik te maken van vloeistofchromatografie gekoppeld aan hoge-resolutie massaspectrometrie zijn we erin geslaagd de eerste geschikte 
bio-analytische bepalingsmethode te ontwikkelen voor de kwantitatieve bepaling van azacitidine-trifosfaat in PBMC's. De bepalingsmethode werd met succes toegepast om de intracellulaire azacitidine-trifosfaat concentraties te meten in PBMC's van twee patiënten die werden behandeld met azacitidine.

Hoofdstuk 3 richt zich op de fluoropyrimidines 5-FU en capecitabine. Drie intracellulair gevormde nucleotiden zijn verantwoordelijk voor het antineoplastisch effect van deze middelen: FUTP, FdUTP en FdUMP. Hoofdstuk 3.1 beschrijft de ontwikkeling van een LC-MS/MS-methode voor de kwantitatieve bepaling van deze drie farmacologisch actieve 5-FU-nucleotiden in PBMC's. Tijdens de ontwikkeling van de methode werd duidelijk dat de 5-FU-nucleotiden concentraties in cellen van patiënten behandeld met 5-FU of capecitabine extreem laag zijn. Dit betekende dat maximalisatie van de afgifte uit de cel-matrix en minimalisatie van interferentie kritische factoren waren om een voldoende gevoelige bepalingsmethode te verkrijgen. Er werd een reeks experimenten uitgevoerd om de beste methode voor cel lysis en nucleotideextractie te selecteren. De chromatografie werd geoptimaliseerd om scheiding te verkrijgen ten opzichte van endogene nucleotiden. Ook het effect van een verschillend aantal geïsoleerde cellen werd onderzocht. De bepalingsmethode werd gevalideerd en met succes toegepast om de 5-FU-nucleotiden te kwantificeren in PBMCs van patiënten die werden behandeld met 5-FU en patiënten behandeld met capecitabine. Dertig minuten na een intraveneuze 5 -FU bolus van $400 \mathrm{mg} / \mathrm{m}^{2}$ werden intracellulaire FUTP hoeveelheden tot $3054 \mathrm{fmol} / 10^{6}$ PBMC's gemeten en FdUMP hoeveelheden tot $169 \mathrm{fmol} / 10^{6}$ PBMC's. De FdUTP concentraties lagen onder de bepalingsgrens van de analysemethode. Dit betekent dat wellicht nog gevoeligere analyseapparatuur nodig is om FdUTP te kunnen meten in patiëntenmonsters. De resultaten voor patiënten die werden behandeld met capecitabine worden hieronder besproken in de paragraaf over hoofdstuk 3.2. Voor zover wij weten, is dit de eerste keer dat 5 -FU-nucleotiden werden gekwantificeerd in cellen van patiënten behandeld met 5-FU of capecitabine zonder een radiolabel te gebruiken. 


\section{De bijdrage van dit proefschrift - Deel 2. Klinische studies waarin de intracellulaire farmacokinetiek wordt onderzocht}

In hoofdstuk 3.2 hebben we de ontwikkelde LC-MS/MS-methode ingezet om de intracellulaire farmacokinetiek van de farmacologisch actieve 5-FU nucleotiden te bestuderen bij patiënten die werden behandeld met capecitabine. Hiertoe werden plasma- en PBMC-monsters verzameld bij 13 patiënten die een eenmalige capecitabine gift van 1000 mg kregen en bij acht patiënten die werden behandeld met tweemaal daags capecitabine $850 \mathrm{mg} / \mathrm{m}^{2}$ gedurende 14 dagen, elke drie weken. De monsters werden verzameld op dag 1 van de behandeling en voor vier patiënten in de laatste groep ook op dag 14 van de behandeling. Alleen FUTP kon worden gemeten in de PBMC's. De FdUTP en FdUMP concentraties lagen onder de detectielimiet van de analysemethode. Blijkbaar wordt capecitabine voornamelijk omgezet in FUTP en in mindere mate in FdUTP en FdUMP. Dit betekent niet persé dat de RNA-route een prominentere rol speelt in het werkingsmechanisme van capecitabine dan de DNA-route. Het is bekend dat ook de natuurlijke deoxyribonucleotiden in veel lagere concentraties in de cel aanwezig zijn dan de ribonucleotiden $[46,47]$. Dit betekent dat lage FdUTP en FdUMP concentraties nog steeds een belangrijke rol kunnen spelen. Echter, voor de kwantificering van FdUMP en FdUTP tijdens de behandeling met capecitabine is wellicht een nog gevoeligere bepalingsmethode nodig.

Dit was de eerste keer dat de intracellulaire FUTP concentraties gemeten werden tijdens de behandeling met capecitabine. Consistent met de eerder bestudeerde pyrimidine analoga werd er geen significante correlatie gevonden tussen de 5-FU plasma blootstelling en de intracellulaire FUTP blootstelling. De intracellulaire FUTP concentratie-tijd profielen toonden grote interindividuele variatie. Een belangrijke bevinding van deze studie was dat FUTP aanzienlijk langer in de cel aanwezig bleef dan capecitabine en 5-FU in plasma. 24 uur na eenmalige inname van capecitabine bedroegen de intracellulaire FUTP concentraties nog steeds $41 \%$ van de piekconcentratie. In overeenstemming met deze bevinding werd na 14 dagen behandeling met tweemaal daags capecitabine accumulatie van FUTP gezien in PBMC's. Het oppervlak onder de FUTP concentratie-tijd curve bleek significant toegenomen op dag 14 van de behandeling ten opzichte van dag 1. Interessant is dat de intracellulaire FUTP concentraties die gemeten werden na 14 dagen behandelen met capecitabine 
in dezelfde orde van grootte lagen als de intracellulaire FUTP concentraties gemeten 30 minuten na een intraveneuze $5-\mathrm{FU}$ bolus van $400 \mathrm{mg} / \mathrm{m}^{2}$.

Dit was de eerste keer dat de intracellulaire farmacokinetiek van de drie farmacologisch actieve 5-FU nucleotiden werd bestudeerd tijdens de behandeling met capecitabine. Natuurlijk moeten we er rekening mee houden dat de nucleotiden concentraties gemeten in PBMC's anders kunnen zijn dan de concentraties in tumorcellen. Zeker omdat capecitabine enige mate van tumorselectiviteit heeft. Het enzym dat verantwoordelijk is voor de derde stap in de omzetting van capecitabine naar 5-FU komt meer voor in tumorweefsel [48].

Hoofdstuk 4.1 richt zich op de intracellulaire farmacokinetiek van gemcitabine. Zoals beschreven aan het begin van dit hoofdstuk, is gemcitabine één van de pyrimidine analoga waarvoor de intracellulaire farmacokinetiek vaker is onderzocht. Verschillende studies maten de intracellulaire gemcitabine-trifosfaat concentraties tijdens behandeling met gemcitabine $[3,4,6,7,9,10,13,14,16$ 18,27]. In plasma wordt gemcitabine snel omgezet in 2',2'-difluoro-2'deoxyuridine (dFdU) door het enzym cytidine deaminase [13]. Vraag is of dFdU ook bijdraagt aan het farmacologisch effect van gemcitabine. Om de rol van $\mathrm{dFdU}$ te verduidelijken, hebben we de intracellulaire farmacokinetiek van zowel de nucleotiden van gemcitabine (d.w.z. gemcitabine-monofosfaat, -difosfaat en -trifosfaat) als de nucleotiden van dFdU (d.w.z. dFdU-monofosfaat, -difosfaat en -trifosfaat) bestudeerd bij patiënten die werden behandeld met gemcitabine. De intracellulaire concentraties werden bepaald in PBMCs afgenomen bij 38 patiënten die werden behandeld met gemcitabine $500-1000 \mathrm{mg} / \mathrm{m}^{2}$, dat in 30 minuten werd toegediend. Hiervoor werd gebruik gemaakt van de LC-MS/MSmethode die eerder door onze groep werd beschreven [49]. Bij alle patiënten was de dFdU plasma blootstelling aanzienlijk hoger dan de gemcitabine plasma blootstelling. Ook in PBMC's was dFdU in hogere concentraties aanwezig dan gemcitabine. dFdU werd echter slechts beperkt gefosforyleerd. De intracellulaire dFdU nucleotiden concentraties in PBMC's waren veel lager dan de gemcitabine nucleotiden concentraties. Dit suggereert dat de bijdrage van dFdU nucleotiden aan het cytotoxisch effect van gemcitabine gering is. Verder onderzoek moet uitwijzen of dFdU ook slechts beperkt gefosforyleerd wordt in tumorcellen. Als dFdU nauwelijks bijdraagt aan het cytotoxisch effect van gemcitabine, door beperkte intracellulaire activatie, kunnen interindividuele verschillen in 
cytidine deaminase activiteit leiden tot substantiële verschillen in de effectiviteit en toxiciteit van gemcitabine therapie tussen patiënten. Dit werd inderdaad gezien in een aantal klinische studies [50-53].

\section{Conclusies}

Met behulp van de nieuwste analytische technieken werden twee geavanceerde bio-analytische bepalingsmethoden ontwikkeld voor kwantitatieve bepaling van de farmacologisch actieve nucleotiden van respectievelijk azacitidine en 5-FU in cellen van patiënten.

$\mathrm{Na}$ behandeling met capecitabine kon alleen FUTP worden gemeten in PBMC's. Blijkbaar wordt capecitabine voornamelijk omgezet in FUTP en in mindere mate in FdUTP en FdUMP. Er was geen duidelijke relatie tussen de 5-FU plasma blootstelling en de intracellulaire FUTP blootstelling. FUTP bleef veel langer aanwezig in PBMC's dan 5-FU in plasma. Gedurende 14 dagen behandeling met tweemaal daags capecitabine trad intracellulaire accumulatie van FUTP op.

$\mathrm{Na}$ behandeling met gemcitabine werden aanzienlijke intracellulaire dFdU concentraties gemeten in PBMC's. dFdU bleek echter maar zeer beperkt gefosforyleerd te worden in PBMC's. De intracellulaire dFdU nucleotiden concentraties waren veel lager dan de gemcitabine nucleotiden concentraties. Dit suggereert dat de bijdrage van dFdU nucleotiden aan het cytotoxisch effect van gemcitabine beperkt is.

Wanneer patiënten werden behandeld met een identieke capecitabine of gemcitabine dosering, werden er aanzienlijke interindividuele verschillen gezien in de intracellulaire nucleotiden blootstelling in PBMC's. Voor een deel zijn deze interindividuele verschillen terug te voeren op verschillen in plasma blootstelling. De interindividuele variatie die werd gezien voor de intracellulaire FUTP, gemcitabine-difosfaat en gemcitabine-trifosfaat blootstelling was echter groter dan de variatie die werd waargenomen in plasma. 


\section{Toekomstperspectieven}

Ondanks de komst van moderne oncolytica, vormen de pyrimidine analoga nog steeds een belangrijke pijler in de behandeling van veel verschillende oncologische aandoeningen. Vaak maken ze al tientallen jaren deel uit van een behandelaanpak. In de loop der jaren zijn behandelregimes geëvolueerd tot de regimes die op dit moment worden toegepast. Niks genuanceerder dan de maximaal getolereerde dosis is meestal uitgangspunt geweest voor deze behandelregimes. Onderzoek dat de farmacologie van de pyrimidine analoga heeft bestudeerd, is vaak decennia geleden uitgevoerd. Inmiddels hebben we veel nieuwe analytische technieken tot onze beschikking. Mijns inziens is het nuttig deze nieuwe analytische technieken in te zetten om de huidige therapie met pyrimidine analoga beter te bestuderen en zo onze kennis over hun klinische farmacologie een update te geven.

Klinisch onderzoek om tot betere behandelregimes te komen, zou mijns inziens veel meer hand in hand moeten gaan met farmacologisch onderzoek. Het is belangrijk om mechanistisch na te denken over wat we zien in onze klinische praktijk en wat we kunnen doen om een therapie te optimaliseren. Situaties waarbij er een kloof ontstaat tussen de kennis van de farmacologie en de klinische praktijk, zoals bij de hoge-dosis cytarabineregimes die tot 2011 gebruikelijk waren, moeten we zien te voorkomen. Precies het tegenovergestelde is waar we naar streven. Nieuwe inzichten in de farmacologie zouden aanleiding moeten zijn om de klinisch toegepaste regimes te verbeteren. Dit vereist samenwerking tussen de verschillende disciplines.

Individualisatie van de therapie is een belangrijk thema in de oncologie en brengt ons steeds meer gerichte therapieën. Vraag is echter waarom we niet meer ons best doen om ook de therapie met klassieke cytostatica meer te individualiseren. Hoofdstuk 3.2 en 4.1 van dit proefschrift hebben laten zien dat wanneer patiënten behandeld worden met een identieke capecitabine of gemcitabine dosering, er aanzienlijke interindividuele verschillen worden gezien in zowel de plasmaspiegels als de intracellulaire nucleotiden spiegels in PBMC's. Dergelijke grote verschillen in spiegels tussen patiënten (zowel in plasma als in cellen) zouden we niet voor lief moeten nemen om pas na een aantal behandelcycli de therapierespons te evalueren. In plaats daarvan zouden we moeten onderzoeken welke spiegels wenselijk zijn; om deze spiegels 
vervolgens na te streven. Immers de grote interindividuele verschillen in plasmaen intracellulaire spiegels zullen betekenen dat een deel van de patiënten niet optimaal behandeld wordt. Wanneer een plasma-effect relatie niet eenduidig kan worden aangetoond, moeten we het daar niet bij laten, maar klinisch farmacologische studies opzetten om uit te zoeken hoe dit komt. Het is niet rationeel om te veronderstellen dat de plasmaspiegel totaal niet relevant is. Het is veel waarschijnlijker dat er nog veel meer factoren bijdragen aan het verschil in klinische uitkomst.

Hoofdstuk 4.1 van dit proefschrift heeft laten zien dat dFdU intracellulair slechts in zeer beperkte mate gefosforyleerd wordt in PBMC's. Toekomstige studies moeten uitwijzen of dFdU ook nauwelijks gefosforyleerd wordt in tumorcellen. Wanneer dat zo is, zal dFdU waarschijnlijk niet substantieel bijdragen aan het cytotoxisch effect van gemcitabine. Het zou dan rationeel zijn om meer aandacht te besteden aan interindividuele verschillen in de afbraak van gemcitabine naar dFdU. Enkele klinische studies hebben al laten zien dat behandeling met gemcitabine effectiever en toxischer was bij patiënten met een lage cytidine deaminase activiteit, met andere woorden een verminderde omzetting van gemcitabine naar dFdU [50-52]. Toekomstige studies zouden moeten uitzoeken of individualisatie van de gemcitabine dosering, op basis van de gemeten cytidine deaminase activiteit, tot betere behandelresultaten leidt.

Op grond van de farmacologie is het waarschijnlijk dat er voor pyrimidine analoga een relatie bestaat tussen de intracellulaire concentraties van de actieve nucleotiden en het cytotoxische effect. Voor de meeste pyrimidine analoga is deze relatie echter alleen aangetoond in in vitro studies en dierstudies [35]. Alleen voor cytarabine is deze relatie bevestigd bij patiënten [33]. Voor de toekomst is het belangrijk om ook voor de andere pyrimidine analoga de relatie tussen de intracellulaire nucleotiden concentraties en het klinisch effect vast te stellen in klinische studies. Hierbij is het nuttig om zowel de relatie te bestuderen tussen de intracellulaire nucleotiden concentraties en de effectiviteit als de relatie tussen de intracellulaire nucleotiden concentraties en toxiciteit. Op deze manier kan een 'intracellulair therapeutisch window' worden gedefinieerd.

Wanneer de relatie tussen de intracellulaire nucleotiden concentraties en het therapeutische effect bij patiënten kan worden bevestigd, biedt dit verschillende 
nieuwe mogelijkheden. Zo zou de intracellulaire nucleotide concentratie in PBMC's gebruikt kunnen worden als voorspellende marker voor het therapeutische effect. Indien de intracellulaire nucleotiden concentraties laag zijn na de eerste gift(en), zou meteen na start van de therapie al kunnen worden gesteld dat er niet veel effect van de therapie met dat pyrimidine analogon, in de gegeven dosering, te verwachten is. Er kan dan een afweging worden gemaakt of het pyrimidine analogon in een hogere dosering wordt gegeven of dat men switcht naar een andere behandeling. Patiënten met te lage intracellulaire nucleotiden concentraties hoeven dan niet langer een aantal, voor hen weinig zinvolle, behandelcycli te ondergaan voordat het effect van de behandeling kan worden geëvalueerd. Dit betekent dat deze patiënten maanden eerder zouden kunnen beginnen met een therapie die, voor hen, potentieel effectiever is. Klinische studies zullen moeten uitwijzen of intracellulaire nucleotiden concentraties inderdaad als voorspellende marker kunnen worden gebruikt.

Als een 'intracellulair therapeutisch window' kan worden gedefinieerd, zou in de toekomst ook de mogelijkheid van therapeutic drug monitoring op basis van de gemeten intracellulaire nucleotide concentratie kunnen worden onderzocht. Op basis van de gemeten intracellulaire nucleotide concentratie en het vastgestelde 'intracellulair therapeutisch window' zou dan een strategie voor dosisaanpassing kunnen worden ontwikkeld.

Gezien al deze mogelijkheden, kan ik niet anders dan stellen dat we, naast het investeren in de ontwikkeling van nieuwe oncolytica, ook onze huidige therapieën onder de loep moeten nemen. Door state-of-the-art analytische technieken te gebruiken, kunnen we onze kennis van de klinische farmacologie een update geven. Deze kennis kan worden gebruikt om de farmacotherapie te verbeteren. Door dit niet te doen, laten we een kans liggen...

Ik heb vertrouwen dat dit proefschrift zal bijdragen aan een beter begrip van de intracellulaire farmacokinetiek van de pyrimidine analoga toegepast in de oncologie. Daarnaast hoop ik dat dit proefschrift een aanzet geeft om de nieuwste bio-analytische technieken in te zetten om meer inzicht te krijgen in de (intracellulaire) farmacologie bij patiënten. Zodat we op basis van state-ofthe-art kennis van de farmacologie, de therapie verder kunnen verbeteren, ook voor de klassieke cytotoxische middelen. 


\section{REFERENCES}

[1] Liliemark JO, Plunkett W, Dixon DO. Relationship of 1-beta-Darabinofuranosylcytosine in plasma to 1-beta-D-arabinofuranosylcytosine 5'-triphosphate levels in leukemic cells during treatment with high-dose 1-betaD-arabinofuranosylcytosine. Cancer Res 1985;45:5952-7.

[2] Plunkett W, Liliemark JO, Adams $T M$, et al. Saturation of 1-beta-Darabinofuranosylcytosine 5'-triphosphate accumulation in leukemia cells during highdose 1-beta-D-arabinofuranosylcytosine therapy. Cancer Res 1987;47:3005-11.

[3] Gandhi V, Plunkett W, Du M, et al. Prolonged infusion of gemcitabine: Clinical and pharmacodynamic studies during a phase I trial in relapsed acute myelogenous leukemia. J Clin Oncol 2002;20:665-673.

[4] Tempero M, Plunkett W, Ruiz van Haperen VW, et al. Randomized Phase II Comparison of Dose-Intense Gemcitabine: Thirty-Minute Infusion and Fixed Dose Rate Infusion in Patients With Pancreatic Adenocarcinoma. J Clin Oncol 2003;21:3402-3408.

[5] Cattel L, Airoldi M, Delprino L, et al. Pharmacokinetic evaluation of gemcitabine and 2',2'-difluorodeoxycytidine-5'triphosphate after prolonged infusion in patients affected by different solid tumors. Ann Oncol 2006;17 Suppl 5:v142-7.

[6] Soo RA, Wang LZ, Tham LS, et al. A multicentre randomised phase II study of carboplatin in combination with gemcitabine at standard rate or fixed dose rate infusion in patients with advanced stage non-small-cell lung cancer. Ann Oncol 2006;17:1128-1133.

[7] Nieto Y, Aldaz A, Rifón J, et al. Phase I and Pharmacokinetic Study of Gemcitabine Administered at Fixed-Dose Rate, Combined with Docetaxel/Melphalan/Carboplatin, with Autologous Hematopoietic ProgenitorCell Support, in Patients with Advanced Refractory Tumors. Biol Blood Marrow Transplant 2007;13:1324-1337.

[8] Veltkamp SA, Jansen RS, Callies S, et al. Oral administration of gemcitabine in patients with refractory tumors: a clinical and pharmacologic study. Clin Cancer Res 2008;14:3477-86.
[9] Poplin E, Feng Y, Berlin J, et al. Phase III, Randomized Study of Gemcitabine and Oxaliplatin Versus Gemcitabine (fixed-dose rate infusion) Compared With Gemcitabine (30-minute infusion) in Patients With Pancreatic Carcinoma E6201: A Trial of the Eastern Cooperative Oncology Group. J Clin Oncol 2009;27:3778-3785.

[10] Caffo O, Fallani S, Marangon E, et al. Pharmacokinetic study of gemcitabine, given as prolonged infusion at fixed dose rate, in combination with cisplatin in patients with advanced non-small-cell lung cancer. Cancer Chemother Pharmacol 2010;65:1197-202.

[11] Plunkett W, Liliemark JO, Estey E, et al. Saturation of ara-CTP accumulation during high-dose ara-C therapy: pharmacologic rationale for intermediate-dose ara-C. Semin Oncol 1987;14:159-66.

[12] Estey EH, Keating MJ, McCredie KB, et al. Cellular ara-CTP pharmacokinetics, response, and karyotype in newly diagnosed acute myelogenous leukemia. Leukemia 1990;4:959.

[13] Abbruzzese JL, Grunewald R, Weeks EA, et al. A phase I clinical, plasma, and cellular pharmacology study of gemcitabine. J Clin Oncol 1991;9:491-8.

[14] Grunewald R, Kantarjian H, Du M, et al. Gemcitabine in leukemia: a phase I clinical, plasma, and cellular pharmacology study. $J$ Clin Oncol 1992;10:406-13.

[15] Gandhi V, Xu YZ, Estey E. Accumulation of arabinosyluracil 5'-triphosphate during arabinosylcytosine therapy in circulating blasts of patients with acute myelogenous leukemia. Clin Cancer Res 1998;4:1719-26.

[16] Kroep JR, Giaccone G, Voorn DA, et al. Gemcitabine and paclitaxel: pharmacokinetic and pharmacodynamic interactions in patients with non-small-cell lung cancer. J Clin Oncol 1999;17:2190-7.

[17] van Moorsel CJ, Kroep JR, Pinedo HM, et al. Pharmacokinetic schedule finding study of the combination of gemcitabine and cisplatin in patients with solid tumors. Ann Oncol 1999;10:441-448. 
[18] Patel SR, Gandhi V, Jenkins J, et al. Phase II clinical investigation of gemcitabine in advanced soft tissue sarcomas and window evaluation of dose rate on gemcitabine triphosphate accumulation. I Clin Oncol 2001;19:3483-9.

[19] Grunewald R, Abbruzzese JL, Tarassoff P, et al. Saturation of 2',2'-difluorodeoxycytidine 5'-triphosphate accumulation by mononuclear cells during a phase I trial of gemcitabine. Cancer Chemother Pharmacol 1991;27:258262.

[20] Farag SS, Ruppert AS, Mrózek K, et al. Outcome of induction and postremission therapy in younger adults with acute myeloid leukemia with normal karyotype: a cancer and leukemia group B study. J Clin Oncol 2005;23:482-93.

[21] Büchner T, Berdel WE, Schoch C, et al. Double induction containing either two courses or one course of high-dose cytarabine plus mitoxantrone and postremission therapy by either autologous stem-cell transplantation or by prolonged maintenance for acute myeloid leukemia. J Clin Oncol 2006;24:2480-2489.

[22] Döhner H, Estey EHE, Amadori S, et al. Diagnosis and management of acute myeloid leukemia in adults: recommendations from an international expert panel, on behalf of the European LeukemiaNet. Blood 2010;115:453474.

[23] Breithaupt $H$, Pralle $H$, Eckhardt $T$, et al. Clinical results and pharmacokinetics of high-dose cytosine arabinoside (HD ARA-C). Cancer 1982;50:1248-57.

[24] Early AP, Preisler HD, Slocum H, et al. A pilot study of high-dose 1-beta-Darabinofuranosylcytosine for acute leukemia and refractory lymphoma: clinical response and pharmacology. Cancer Res 1982;42:1587-94.

[25] Capizzi RL, Yong JL, Cheng E. Alteration of the pharmacokinetics of high-dose Ara-C by its metabolite, high Ara- $U$ in patients with acute leukemia. J Clin Oncol 1983;1:763-771.

[26] Löwenberg B, Pabst T, Vellenga E, et al. Cytarabine dose for acute myeloid leukemia. N Engl J Med 2011;364:1027-1036.

[27] Cattel L, Airoldi M, Delprino L, et al. Pharmacokinetic evaluation of gemcitabine and 2',2'-difluorodeoxycytidine-5'triphosphate after prolonged infusion in patients affected by different solid tumors. Ann Oncol 2006;17 Suppl 5:v142-7.
[28] Gridelli C, Gallo C, Ceribelli A, et al. Factorial phase III randomised trial of rofecoxib and prolonged constant infusion of gemcitabine in advanced non-small-cell lung cancer: the GEmcitabine-COxib in NSCLC (GECO) study. Lancet Oncol 2007;8:500-512.

[29] Fossella F V, Lippman SM, Shin DM, et al. Maximum-tolerated dose defined for singleagent gemcitabine: a phase I dose-escalation study in chemotherapy-naive patients with advanced non-small-cell lung cancer. J Clin Oncol 1997;15:310-6.

[30] Brand R, Capadano M, Tempero M. A phase I trial of weekly gemcitabine administered as a prolonged infusion in patients with pancreatic cancer and other solid tumors. Invest New Drugs 1997;15:331-341.

[31] Touroutoglou N, Gravel D, Raber MN, et al. Clinical results of a pharmacodynamicallybased strategy for higher dosing of gemcitabine in patients with solid tumors. Ann Oncol 1998;9:1003-1008.

[32] Kantarjian HM, Estey EH, Plunkett W, et al. Phase I-II clinical and pharmacologic studies of high-dose cytosine arabinoside in refractory leukemia. Am J Med 1986;81:387394.

[33] Estey E, Plunkett W, Dixon D, et al. Variables predicting response to high dose cytosine arabinoside therapy in patients with refractory acute leukemia. Leukemia 1987;1:580-3.

[34] Grimison P, Galettis P, Manners S, et al. Randomized crossover study evaluating the effect of gemcitabine infusion dose rate: evidence of auto-induction of gemcitabine accumulation. J Clin Oncol 2007;25:5704-9.

[35] Ruiz van Haperen VW, Veerman G, Boven $E$, et al. Schedule dependence of sensitivity to 2',2'-difluorodeoxycytidine (gemcitabine) in relation to accumulation and retention of its triphosphate in solid tumour cell lines and solid tumours. Biochem Pharmacol 1994;48:1327-1339.

[36] Peters GJ, Laurensse E, Leyva A, et al. Sensitivity of human, murine, and rat cells to 5-fluorouracil and 5'-deoxy-5-fluorouridine in relation to drug-metabolizing enzymes. Cancer Res 1986;46:20-8.

[37] Ciccolini J, Peillard L, Evrard A, et al. Enhanced antitumor activity of 5-fluorouracil in combination with 2'-deoxyinosine in human colorectal cell lines and human colon tumor xenografts. Clin Cancer Res 2000;6:15291535. 
[38] Ciccolini J, Peillard L, Aubert C, et al. Monitoring of the intracellular activation of 5-fluorouracil to deoxyribonucleotides in HT29 human colon cell line: application to modulation of metabolism and cytotoxicity study. Fundam Clin Pharmacol 2000;14:147154.

[39] Kamm YJL, Peters GJ, Hull WE, et al. Correlation between 5-fluorouracil metabolism and treatment response in two variants of $\mathrm{C} 26$ murine colon carcinoma. $\mathrm{Br} J$ Cancer 2003;89:754-62.

[40] Spears CP, Gustavsson BG, Mitchell MS, et al. Thymidylate synthetase inhibition in malignant tumors and normal liver of patients given intravenous 5-fluorouracil. Cancer Res 1984;44:4144-50.

[41] Spears CP, Gustavsson BG, Berne $M$, et al. Mechanisms of innate resistance to thymidylate synthase inhibition after 5-fluorouracil. Cancer Res 1988;48:58945900.

[42] Peters GJ, Lankelma J, Kok RM, et al. Prolonged retention of high concentrations of 5-fluorouracil in human and murine tumors as compared with plasma. Cancer Chemother Pharmacol 1993;31:269-76.

[43] Moran RG, Spears CP, Heidelberger C. Biochemical determinants of tumor sensitivity to 5-fluorouracil: ultrasensitive methods for the determination of 5-fluoro2'-deoxyuridylate, 2'-deoxyuridylate, and thymidylate synthetase. Proc Natl Acad SciU SA 1979;76:1456-60.

[44] Jansen RS, Rosing H, Wijermans PW, et al. Decitabine triphosphate levels in peripheral blood mononuclear cells from patients receiving prolonged low-dose decitabine administration: A pilot study. Cancer Chemother Pharmacol 2012;69:1457-1466.

[45] Wang $\mathrm{H}$, Chen $\mathrm{P}$, Wang J, et al. In vivo quantification of active decitabinetriphosphate metabolite: a novel pharmacoanalytical endpoint for optimization of hypomethylating therapy in acute myeloid leukemia. AAPS J 2013;15:242-9.
[46] Chen P, Liu Z, Liu S, et al. A LC-MS/MS method for the analysis of intracellular nucleoside triphosphate levels. Pharm Res 2009;26:1504-15.

[47] Cohen S, Megherbi M, Jordheim LP, et al. Simultaneous analysis of eight nucleoside triphosphates in cell lines by liquid chromatography coupled with tandem mass spectrometry. J Chromatogr B Analyt Technol Biomed Life Sci 2009;877:3831-40.

[48] Miwa M, Ura M, Nishida M, et al. Design of a novel oral fluoropyrimidine carbamate, capecitabine, which generates 5 -fluorouracil selectively in tumours by enzymes concentrated in human liver and cancer tissue. Eur J Cancer 1998;34:1274-81.

[49] Jansen RS, Rosing $H$, Schellens JHM, et al. Simultaneous quantification of 2',2'-difluorodeoxycytidine and 2',2'-difluorodeoxyuridine nucleosides and nucleotides in white blood cells using porous graphitic carbon chromatography coupled with tandem mass spectrometry. Rapid Commun Mass Spectrom 2009;23:3040-50.

[50] Ciccolini J, Dahan L, André N, et al. Cytidine deaminase residual activity in serum is a predictive marker of early severe toxicities in adults after gemcitabine-based chemotherapies. J Clin Oncol 2010;28:160165.

[51] Tibaldi C, Giovannetti E, Vasile E, et al. Correlation of CDA, ERCC1, and XPD polymorphisms with response and survival in gemcitabine/cisplatin-treated advanced nonsmall cell lung cancer patients. Clin Cancer Res 2008;14:1797-1803.

[52] Tibaldi C, Giovannetti E, Tiseo M, et al. Correlation of cytidine deaminase polymorphisms and activity with clinical outcome in gemcitabine-/platinum-treated advanced non-small-cell lung cancer patients. Ann Oncol 2012;23:670-677.

[53] Serdjebi C, Seitz J-F, Ciccolini J, et al. Rapid deaminator status is associated with poor clinical outcome in pancreatic cancer patients treated with a gemcitabine-based regimen. Pharmacogenomics 2013;14:1047-51. 
CHEMICAL STRUCTURES OF THE PYRIMIDINE ANALOGUES DISCUSSED IN THIS THESIS

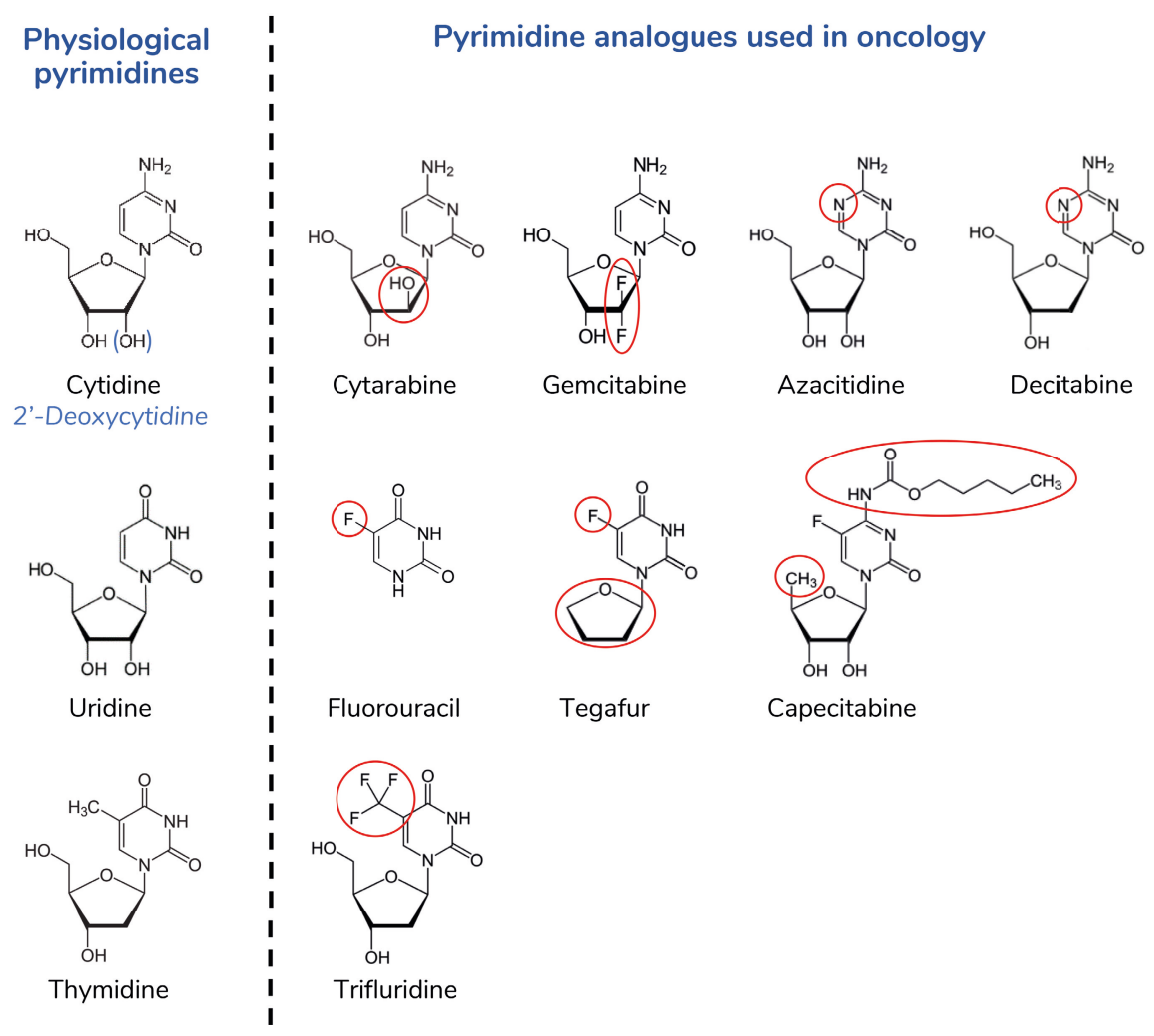




\section{List of Publications Dankwoord Curriculum Vitae}

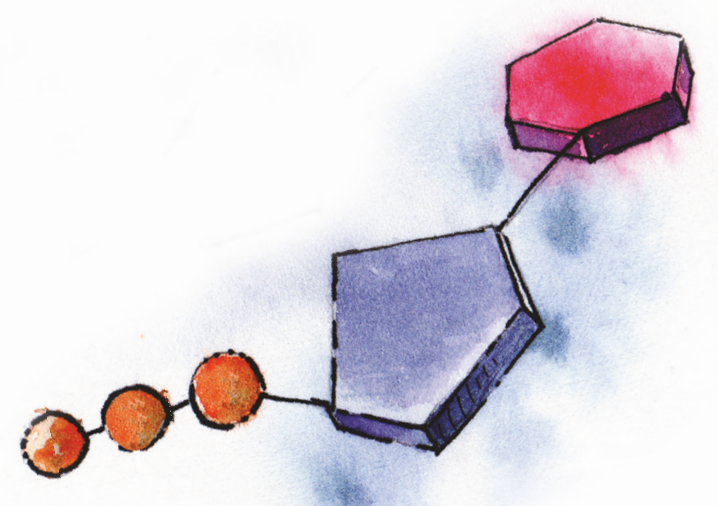




\section{LIST OF PUBLICATIONS}

Derissen EJB, Beijnen JH. Intracellular pharmacokinetics of pyrimidine analogues used in oncology and the correlation with drug action. - Review Article - Submitted for publication

Derissen EJB, Huitema ADR, Rosing $H$, Schellens JHM, Beijnen JH. Intracellular pharmacokinetics of gemcitabine, its deaminated metabolite 2',2'-difluorodeoxyuridine and their nucleotides. $\mathrm{Br} J$ Clin Pharmacol 2018;84:1279-1289.

Derissen EJB, Jacobs BAW, Huitema ADR, Rosing H, Schellens JHM, Beijnen $\mathrm{JH}$. Exploring the intracellular pharmacokinetics of the 5-fluorouracil nucleotides during capecitabine treatment. Br J Clin Pharmacol 2016;81:949-957.

Derissen EJB, Hillebrand MJX, Rosing $\mathrm{H}$, Schellens JHM, Beijnen JH. Development of an LC-MS/MS assay for the quantitative determination of the intracellular 5-fluorouracil nucleotides responsible for the anticancer effect of 5-fluorouracil. J Pharm Biomed Anal 2015;110:58-66.

Derissen EJB, Hillebrand MJX, Rosing H, Otten HMMB, Laille E, Schellens JHM, Beijnen JH. Quantitative determination of azacitidine triphosphate in peripheral blood mononuclear cells using liquid chromatography coupled with highresolution mass spectrometry. J Pharm Biomed Anal 2014;90:7-14.

Derissen EJB, Beijnen JH, Schellens JHM. Concise drug review: azacitidine and decitabine. Oncologist 2013;18:619-624.

Manson ML, Derissen EJB, Wijermans PW, Schellens JHM, Beijnen JH. Demethylating medication in myelodysplastic syndrome. Ned Tijdschr Geneeskd 2012;156:A3167.

Damen CWN, Derissen EJB, Schellens JHM, Rosing H, Beijnen JH. The bioanalysis of the monoclonal antibody trastuzumab by high-performance liquid chromatography with fluorescence detection after immuno-affinity purification from human serum. J Pharm Biomed Anal 2009;50:861-866. 


\section{DANKWOORD}

Het is zover... het boekje is klaar! Vele mooie jaren heb ik aan dit onderzoek mogen werken. Deze jaren waren zo leuk, omdat ik het niet alleen heb gedaan maar met jullie. De laatste bladzijden van dit proefschrift wil ik dan ook graag gebruiken om jullie allemaal te bedanken.

Allereerst wil ik de patiënten bedanken die zonder eigenbelang hebben meegewerkt aan de studies beschreven in dit proefschrift. Dank voor alle bloedafnames. Zonder jullie was het niet mogelijk geweest de intracellulaire kinetiek van de verschillende pyrimidine analoga te bestuderen.

Natuurlijk wil ik ook mijn promotor Jos Beijnen en copromotor Hilde Rosing bedanken. Beste Jos, dank voor de mogelijkheid die je me hebt geboden om dit interessante onderzoek te doen in de inspirerende omgeving van het NKI en het Slotervaartziekenhuis. Dank ook voor het blijven meedenken over mijn onderzoek en de ruimte en het vertrouwen die je me gaf om het onderzoek, ook na mijn tijd in het Slotervaart, af te ronden. Beste Hilde, dank voor je betrokkenheid en het meedenken over de verschillende experimenten die ik nog kon doen om stap voor stap te komen tot bruikbare analysemethoden.

Ook Jan Schellens en Alwin Huitema wil ik hartelijk danken voor de begeleiding. Beste Jan, dank voor je waardevolle input zowel bij mijn onderzoek als ook als opleider voor de klinische farmacologie opleiding. Beste Alwin, dank voor het kritisch bekijken van mijn artikelen over de intracellulaire PK van de pyrimidine analoga en je waardevolle commentaar.

De leden van de beoordelingscommissie wil ik hartelijk danken voor het beoordelen van dit proefschrift.

Mijn tijd in het Slotervaartziekenhuis begon als stagiaire tijdens de master Farmacie. Carola, - mijn stagebegeleidster destijds - dank dat je me als stagiaire wegwijs heb gemaakt op het lab en binnen de onderzoeksgroep. Na deze stage wilde ik niet meer weg en was het logisch dat ik bleef voor promotieonderzoek. Robert, jij had al een stevige basis gelegd aan het nucleotiden onderzoek en ik 
mocht het stokje van je overnemen. Dank voor je hulp het eerste jaar. Dankzij jou was ik snel ingewijd in de wereld van de PBMCs en de trifosfaten.

Ook de collega's van het lab - Abadi, Bas B, Bas T, Ciska, Dieuwke, Joke, Kees, Lianda, Luc, Matthijs, Michel en Niels - wil ik hartelijk bedanken voor alle support en de plezierige werkomgeving. Beste Michel, dank voor je hulp bij het ingewikkeldere LC-MS/MS werk. Geen heen en weer ritjes meer in de 'pausmobiel' tussen het Slotervaart en het NKI om een HPLC-opstelling richting de Orbitrap te transporteren. Sasha, many thanks that we were allowed to use your Orbitrap. Beste Matthijs, bedankt voor het teamwork voor de verschillende gemcitabine-trifosfaat studies. Roel en Denise, dank voor jullie waardevolle bijdrage als Qa-ers.

Natuurlijk wil ik ook alle coauteurs danken voor de waardevolle bijdragen. Bart Jacobs wil ik bedanken voor de goede samenwerking tijdens het capecitabine onderzoek en Ruud van der Noll voor de samenwerking bij het gemcitabine onderzoek. Dick Pluim wil ik hartelijk danken voor de Bradford metingen en later voor de hulp bij de experimenten om te corrigeren voor het hemoglobine gehalte. De collega's van klinische chemie wil ik danken dat ik steeds celtellingen bij hen mocht komen uitvoeren.

Anne-Charlotte, Annemieke, Bas, Corine, David, Jelte, Johannes, Jolanda, Joost, Ly, Mariska, Nienke, Rik, Rob, Robert, Ron, Stijn, Susanne, Thomas, Tine, Wiete, Anita, Coen, Cynthia, Emilia, Iris, Jeroen H, Nalini, Nynke, Anke, Aurelia, Huixin, Jeroen R, Julie, Lotte, Maikel, Merel, Remy, Rose en Sven - alle OIO's met wie ik in de loop der jaren in 'de keet' heb mogen vertoeven - en daarbij natuurlijk ook alle OIO's van het NKI, dank voor de gezelligheid, de vieruurtjes, de vrimibo's, de etentjes en alle OIO-weekenden die ik met jullie heb mogen beleven. In het bijzonder wil ik mijn kamergenoten bedanken. Wiete en Nienke, mijn roomies van het eerste uur, ik vond het supergezellig om samen met jullie op een kamer te zitten en een groot gedeelte van ons promotietraject samen te doen. Mijn latere kamergenoot Jeroen, dank voor de gezelligheid. Hoog tijd om weer eens wat 'bokalen met straaljager' te preparen voor alle collega's van toen. Wiete, Nienke, Nynke, Anke, Aurelia en later ook alle andere matties die aansloten..., dank voor jullie mentale support tijdens het dagelijkse 4-uurtje... en daarbuiten. Ik vond het super gezellig om samen OlO te zijn geweest in 'de keet'. 
Mijn collega's van het VUmc wil ik hartelijk danken voor de plezierige werkomgeving op dit moment. Wat fijn dat ik, na mijn promotietijd in het NKI, bij jullie de opleiding tot ziekenhuisapotheker mag doen! Dank ook voor jullie begrip voor de effort die nodig was voor de afronding van mijn proefschrift naast de opleiding tot ziekenhuisapotheker, jullie collegialiteit en de flexibiliteit om zo nu en dan een (dag)dienst te ruilen.

Emily en Nynke, dank dat jullie mij ook tijdens de verdediging van mijn proefschrift willen bijstaan en mijn paranimfen willen zijn.

Lieve Pap, Mam en Emily, dank voor ALLES... veel meer dan wat je in zo'n dankwoord kunt vatten. Ik had me geen betere ouders en zus kunnen wensen!

Ellen 


\section{CURRICULUM VITAE}

Ellen Derissen was born on January $23^{\text {rd }} 1984$ in Zeist and grew up in Oisterwijk, the Netherlands. After high school graduation at the Sint Odulphuslyceum in Tilburg in 2002, she started studying Pharmacy at Utrecht University. During the Bachelor's programme, she studied the changes in hepatorenal drug clearance in the elderly. In 2006 she received her BSc degree. During the Master's programme, she performed a scientific internship at the Slotervaart Hospital and the Netherlands Cancer Institute in Amsterdam. Here she developed

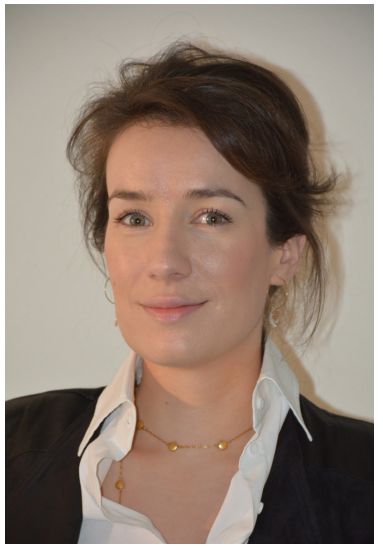
a bio-analytical assay for the quantitative determination of trastuzumab in serum. After receiving her MSc degree in 2009, she started her PhD research at the Department of Pharmacy \& Pharmacology of the Netherlands Cancer Institute - Antoni van Leeuwenhoek Hospital in Amsterdam, supervised by Prof. dr. J.H. Beijnen, Prof. dr. J.H.M. Schellens and dr. H. Rosing. Her PhD research resulted in this thesis about the intracellular quantification and pharmacokinetics of pyrimidine analogues used in oncology. In addition to her PhD program, Ellen was also trained to become a Clinical Pharmacologist. Certification was granted in 2015 by the Dutch Society for Clinical Pharmacology \& Biopharmacy (NVKFB). Since April 2016 Ellen works as a Hospital Pharmacist in training at the Amsterdam University Medical Center, location VUmc. During the training programme she also worked at the OLVG Hospital in Amsterdam. At the end of March 2020, Ellen expects to complete the training as a Hospital Pharmacist. 